\title{
2019 ESC/EAS guidelines for the management of dyslipidaemias: Lipid modification to reduce cardiovascular risk ${ }^{\text {in }}$
}

Authors/Task Force Members (François Mach ${ }^{\mathrm{a}, *, 2}$, Colin Baigent ${ }^{\mathrm{b}, * *, 2}$, Alberico L. Catapano ${ }^{\mathrm{c}, * *, 1,2}$, Konstantinos C. Koskinas ${ }^{\mathrm{d}}$, Manuela Casula ${ }^{\mathrm{e}, \mathrm{f}, 1}$, Lina Badimon ${ }^{g}$, M. John Chapman ${ }^{\mathrm{h}, \mathrm{i}, \mathrm{cm}, 1}$, Guy G. De Backer ${ }^{\mathrm{j}}$, Victoria Delgado ${ }^{\mathrm{k}}$, Brian A. Ference ${ }^{\mathrm{l}}$, Ian M. Graham ${ }^{\mathrm{m}}$, Alison Halliday ${ }^{\mathrm{n}}$, Ulf Landmesser ${ }^{\mathrm{o}, \mathrm{p}, \mathrm{q}}$, Borislava Mihaylova ${ }^{\mathrm{r}, \mathrm{s}}$, Terje R. Pedersen ${ }^{\mathrm{t}}$, Gabriele Riccardi ${ }^{\mathrm{u}, 1}$, Dimitrios J. Richter ${ }^{\mathrm{v}}$, Marc S. Sabatine ${ }^{\mathrm{w}}$, Marja-Riitta Taskinen $^{\mathrm{x}, 1}$, Lale Tokgozoglu ${ }^{\mathrm{y}, 1}$, Olov Wiklund ${ }^{\mathrm{z}}$ ), ESC National Cardiac Societies (Djamaleddine Nibouche $^{\text {an }}$, Parounak H. Zelveian ${ }^{\text {ao }}$, Peter Siostrzonek ${ }^{\text {ap }}$, Ruslan Najafov ${ }^{\text {aq }}$, Philippe van de Borne ${ }^{\text {ar }}$, Belma Pojskic ${ }^{\text {as }}$, Arman Postadzhiyan ${ }^{\text {at }}$, Lambros Kypris ${ }^{\text {au }}$, Jindřich Špinar $^{\text {av }}$, Mogens Lytken Larsen ${ }^{\text {aw }}$, Hesham Salah Eldin ${ }^{\text {ax }}$, Margus Viigimaa ${ }^{\text {ay }}$, Timo E. Strandbergaz, Jean Ferrières ${ }^{\mathrm{ba}}$, Rusudan Agladze ${ }^{\mathrm{bb}}$, Ulrich Laufs ${ }^{\mathrm{bc}}$, Loukianos Rallidis ${ }^{\mathrm{bd}}$, László Bajnok $^{\text {be }}$, Thorbjörn Gudjónsson ${ }^{\text {bf }}$, Vincent Maher ${ }^{\text {bg }}$, Yaakov Henkin ${ }^{\text {bh }}$, Michele Massimo Gulizia $^{\text {bi }}$, Aisulu Mussagaliyeva ${ }^{\text {bj }}$, Gani Bajraktari ${ }^{\text {bk }}$, Alina Kerimkulova ${ }^{\text {bl }}$, Gustavs Latkovskis $^{\mathrm{bm}}$, Omar Hamoui ${ }^{\mathrm{bn}}$, Rimvydas Slapikas ${ }^{\mathrm{bo}}$, Laurent Visser ${ }^{\mathrm{bp}}$, Philip Dingli ${ }^{\mathrm{bq}}$, Victoria Ivanov $^{\text {br }}$, Aneta Boskovic ${ }^{\text {bs }}$, Mbarek Nazzi ${ }^{\text {bt }}$, Frank Visseren ${ }^{\text {bu }}$, Irena Mitevska ${ }^{\text {bv }}$, Kjetil Retterst $\varnothing 1^{\text {bw }}$, Piotr Jankowski ${ }^{\text {bx }}$, Ricardo Fontes-Carvalho ${ }^{\text {by }}$, Dan Gaita ${ }^{\text {bz }}$, Marat Ezhov ${ }^{\text {ca }}$, Marina Foscoli ${ }^{\mathrm{cb}}$, Vojislav Giga ${ }^{\mathrm{cc}}$, Daniel Pella ${ }^{\mathrm{cd}}$, Zlatko Fras ${ }^{\mathrm{ce}}$, Leopoldo Perez de Isla ${ }^{\mathrm{cf}}$, Emil Hagström $^{\mathrm{cg}}$, Roger Lehmann ${ }^{\text {ch }}$, Leila Abid ${ }^{\text {ci }}$, Oner Ozdogan ${ }^{\mathrm{cj}}$, Olena Mitchenko ${ }^{\text {ck }}$, Riyaz S. Patel ${ }^{\mathrm{cl}}$ ), ESC Committee for Practice Guidelines (CPG) (Stephan Windecker ${ }^{\text {aa }}$, Victor Aboyans $^{\mathrm{ab}}$, Colin Baigent ${ }^{\mathrm{ac}}$, Jean-Philippe Collet ${ }^{\mathrm{ab}}$, Veronica Dean ${ }^{\mathrm{ab}}$, Victoria Delgado ${ }^{\mathrm{ad}}$, Donna Fitzsimons $^{\text {ac }}$, Chris P. Gale ${ }^{\text {ac }}$, Diederick Grobbee ${ }^{\text {ad }}$, Sigrun Halvorsen ${ }^{\text {ae }}$, Gerhard Hindricks $^{\text {af }}$, Bernard Iung ${ }^{\mathrm{ab}}$, Peter Jüni ${ }^{\mathrm{ag}}$, Hugo A. Katus ${ }^{\text {af }}$, Ulf Landmesser ${ }^{\text {af }}$, Christophe Leclercq $^{\text {ab }}$, Maddalena Lettino ${ }^{\text {ah }}$, Basil S. Lewis ${ }^{\text {ai }}$, Bela Merkely ${ }^{\text {aj }}$, Christian Mueller ${ }^{\text {aa }}$, Steffen Petersen ${ }^{\text {ac }}$, Anna Sonia Petronio ${ }^{\text {ah }}$, Dimitrios J. Richter ${ }^{\text {ak }}$, Marco Roffi ${ }^{\text {aa }}$, Evgeny Shlyakhto $^{\text {al }}$, Iain A. Simpson ${ }^{\text {ac }}$, Miguel Sousa-Uva ${ }^{\text {am }}$, Rhian M. Touyz ${ }^{\text {ac}}$ )

${ }^{a}$ Cardiology Department, Geneva University Hospital, 4 Gabrielle-Perret-Gentil, 1211, Geneva, Switzerland

${ }^{\mathrm{b}}$ Nuffield Department of Population Health, University of Oxford, Richard Doll Building, Roosevelt Drive, Oxford OX3 7LF, United Kingdom

${ }^{\mathrm{c}}$ Department of Pharmacological and Biomolecular Sciences, University of Milan, Multimedica IRCCS, Via Balzaretti, 9, 20133, Milan, Italy

${ }^{\mathrm{d}}$ Cardiology, Bern University Hospital (Inselspital), Bern, Switzerland

${ }^{\mathrm{e}}$ Epidemiology and Preventive Pharmacology Service (SEFAP), Department of Pharmacological and Biomolecular Sciences (DiSFeB), University of Milan, Milan, Italy

${ }^{\mathrm{f}}$ IRCCS MultiMedica, Sesto S. Giovanni, Milan, Italy

${ }^{g}$ Cardiovascular Program-ICCC and CiberCV, IR-Hospital de la Santa Creu i Sant Pau, Barcelona, Spain

${ }^{\mathrm{h}}$ National Institute for Health and Medical Research (INSERM), Paris, France

${ }^{i}$ Division of Endocrinology-Metabolism, Pitie-Salpetriere University Hospital, Paris, France

${ }^{\mathrm{j}}$ Public Health and Primary Care, Faculty of Medicine and Health Sciences, Ghent University, Ghent, Belgium

${ }^{\mathrm{k}}$ Cardiology, Leiden University Medical Center, Leiden, Netherlands

${ }^{1}$ Centre for Naturally Randomized Trials, Department of Public Health and Primary Care, University of Cambridge, Cambridge, United Kingdom

${ }^{\mathrm{m}}$ Cardiology, Trinity College, Dublin, Ireland

\footnotetext{
The Task Force for the management of dyslipidaemias of the European Society of Cardiology (ESC) and European Atherosclerosis Society (EAS). The disclosure forms of all experts involved in the development of these Guidelines are available on the ESC website www.escardio.org/guidelines. * Corresponding author.

** Corresponding author.

${ }^{* * * *}$ Corresponding author.
} 
${ }^{\mathrm{n}}$ Nuffield Department of Surgery, University of Oxford, NIHR Oxford Biomedical Research Centre, Oxford, United Kingdom

${ }^{\circ}$ Department of Cardiology, Charite Universitätsmedizin Berlin, Berlin, Germany

${ }^{\mathrm{P}}$ Berlin Institute of Health (BIH), Berlin, Germany

${ }^{\mathrm{q}}$ German Center of Cardiovascular Research (DZHK), Berlin, Germany

${ }^{\mathrm{r}}$ Nuffield Department of Population Health, NIHR Oxford Biomedical Research Centre, University of Oxford, Oxford, United Kingdom

${ }^{\mathrm{s}}$ Barts and the London School of Medicine and Dentistry, Queen Mary University of London, London, United Kingdom

${ }^{\mathrm{t}}$ Preventive Cardiology, Oslo University Hospital, Aker, Oslo, Norway

${ }^{\mathrm{u}}$ Clinical Medicine and Surgery, Federico II University, Naples, Italy

${ }^{v}$ Cardiac Department, Euroclinic, Athens, Greece

${ }^{\mathrm{w}}$ TIMI Study Group, Division of Cardiovascular Medicine, Brigham, Women's Hospital and Harvard Medical School, Boston, MA, United States of America

${ }^{\mathrm{x}}$ Research Program for Clinical and Molecular Metabolism, University of Helsinki, Helsinki, Finland

${ }^{y}$ Cardiology, Hacettepe University, Ankara, Turkey

${ }^{\mathrm{z}}$ Institute of Medicine, The Sahlgrenska Academy at University of Gothenburg, Gothenburg, Sweden

${ }^{\mathrm{cm}}$ Sorbonne University, Paris, France

${ }^{\text {aa }}$ Switzerland

${ }^{\mathrm{ab}}$ France

ac United Kingdom

${ }^{\text {ad }}$ Netherlands

${ }^{\text {ae }}$ Norway

${ }^{\text {af Germany }}$

${ }^{\text {ag }}$ Canada

${ }^{\text {ah }}$ Italy

${ }^{\text {ai }}$ Israel

${ }^{\text {aj }}$ Hungary

${ }^{\mathrm{ak}}$ Greece

${ }^{\text {al }}$ Russian Federation

${ }^{\text {am }}$ Portugal

${ }^{\text {an }}$ Algerian Society of Cardiology, Algeria

${ }^{\text {ao }}$ Armenian Cardiologists Association, Armenia

${ }^{\text {ap }}$ Austrian Society of Cardiology, Austria

${ }^{\mathrm{aq}}$ Azerbaijan Society of Cardiology, Azerbaijan

${ }^{\text {ar }}$ Belgian Society of Cardiology, Belgium

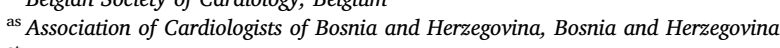

at Bulgarian Society of Cardiology, Bulgaria

${ }^{\text {au }}$ Cyprus Society of Cardiology, Cyprus

${ }^{a v}$ Czech Society of Cardiology, Czech Republic

${ }^{\text {aw }}$ Danish Society of Cardiology, Denmark

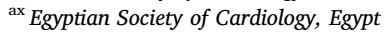

${ }^{\text {ay }}$ Estonian Society of Cardiology, Estonia

${ }^{\mathrm{az}}$ Finnish Cardiac Society, Finland

${ }^{\text {ba }}$ French Society of Cardiology, France

${ }^{\mathrm{bb}}$ Georgian Society of Cardiology, Georgia

bc German Cardiac Society, Germany

${ }^{b d}$ Hellenic Society of Cardiology, Greece

be Hungarian Society of Cardiology, Hungary

${ }^{\text {bf }}$ Icelandic Society of Cardiology, Iceland

bg Irish Cardiac Society, Ireland

${ }^{\text {bh }}$ Israel Heart Society, Israel

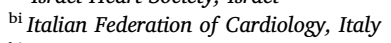

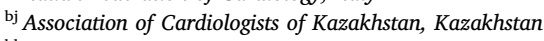

${ }^{\mathrm{bk}}$ Kosovo Society of Cardiology, Republic of Kosovo

${ }^{\mathrm{bl}}$ Kyrgyz Society of Cardiology, Kyrgyzstan

${ }^{\mathrm{bm}}$ Latvian Society of Cardiology, Latvia

bn Lebanese Society of Cardiology, Lebanon

bo Lithuanian Society of Cardiology, Lithuania

${ }^{\text {bp }}$ Luxembourg Society of Cardiology, Luxembourg

${ }^{\mathrm{bq}}$ Maltese Cardiac Society, Malta

${ }^{\mathrm{br}}$ Moldavian Society of Cardiology, Republic of Moldova

${ }^{\text {bs }}$ Montenegro Society of Cardiology, Montenegro

${ }^{\mathrm{bt}}$ Moroccan Society of Cardiology, Morocco

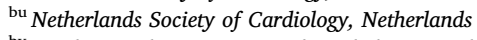

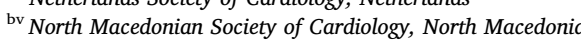

${ }^{\mathrm{bw}}$ Norwegian Society of Cardiology, Norway

${ }^{\mathrm{bx}}$ Polish Cardiac Society, Poland

by Portuguese Society of Cardiology, Portugal

${ }^{\mathrm{bz}}$ Romanian Society of Cardiology, Romania

${ }^{\text {ca }}$ Russian Society of Cardiology, Russian Federation

${ }^{\mathrm{cb}}$ San Marino Society of Cardiology, San Marino

${ }^{\mathrm{cc}}$ Cardiology Society of Serbia, Serbia

${ }^{\mathrm{cd}}$ Slovak Society of Cardiology, Slovakia

ce Slovenian Society of Cardiology, Slovenia

${ }^{\mathrm{cf}}$ Spanish Society of Cardiology, Spain

${ }^{\mathrm{cg}}$ Swedish Society of Cardiology, Sweden

${ }^{\text {ch }}$ Swiss Society of Cardiology, Switzerland 
${ }^{c i}$ Tunisian Society of Cardiology and Cardio-Vascular Surgery, Tunisia

${ }^{\mathrm{cj}}$ Turkish Society of Cardiology, Turkey

${ }^{\mathrm{ck}}$ Ukrainian Association of Cardiology, Ukraine

${ }^{\mathrm{cl}}$ British Cardiovascular Society, United Kingdom of Great Britain and Northern Ireland

A R T I C L E I N F O

Keywords:

Guidelines

Dyslipidaemias

Cholesterol;

Triglycerides

Low-density lipoproteins

High-density lipoproteins

Apolipoprotein B

Lipoprotein(a)

Lipoprotein remnants

Total cardiovascular risk

Treatment (lifestyle)

Treatment (drugs)

Treatment (adherence)

Very low-density lipoproteins

Familial hypercholesterolaemia

\section{Preamble}

Guidelines summarize and evaluate available evidence with the aim of assisting health professionals in proposing the best management strategies for an individual patient with a given condition. Guidelines and their recommendations should facilitate decision making of health professionals in their daily practice. However, the final decisions concerning an individual patient must be made by the responsible health professional(s) in consultation with the patient and caregiver as appropriate. (see Table 1)

A great number of guidelines have been issued in recent years by the European Society of Cardiology (ESC) and its partners such as European Atherosclerosis Society (EAS), as well as by other societies and organisations. Because of their impact on clinical practice, quality criteria for the development of guidelines have been established in order to make all decisions transparent to the user. The recommendations for formulating and issuing ESC Guidelines can be found on the ESC website (http://www.escardio.org/Guidelines-\&-Education/ClinicalPractice-Guidelines/Guidelines-development/Writing-ESC-Guidelines). The ESC Guidelines represent the official position of the ESC on a given topic and are regularly updated.

The ESC carries out a number of registries which are essential to assess diagnostic/therapeutic processes, use of resources and adherence to Guidelines. These registries aim at providing a better understanding of medical practice in Europe and around the world, based on data collected during routine clinical practice.

The guidelines are developed together with derivative educational material addressing the cultural and professional needs for cardiologists and allied professionals. Collecting high-quality observational data, at appropriate time interval following the release of ESC Guidelines, will help evaluate the level of implementation of the Guidelines, checking in priority the key end points defined with the ESC Guidelines and Education Committees and Task Force members in charge.

The Members of this Task Force were selected by the ESC and EAS, including representation from relevant ESC sub-specialty groups, in order to represent professionals involved with the medical care of patients with this pathology. Selected experts in the field from both societies undertook a comprehensive review of the published evidence for management

\footnotetext{
${ }^{1}$ Representing the EAS.

${ }^{2}$ The three chairpersons contributed equally to the document.
}

of a given condition according to ESC Committee for Practice Guidelines (CPG) policy. A critical evaluation of diagnostic and therapeutic procedures was performed, including assessment of the risk-benefit ratio. The level of evidence and the strength of the recommendation of particular management options were weighed and graded according to predefined ESC scales, as outlined in the tables below.

The experts of the writing and reviewing panels provided declaration of interest forms for all relationships that might be perceived as real or potential sources of conflicts of interest. These forms were compiled into one file and can be found on the ESC website (http:// www.escardio.org/guidelines). Any changes in declarations of interest that arise during the writing period were notified to the ESC and EAS Chairpersons and updated. The Task Force received its entire financial support from the ESC and EAS without any involvement from the healthcare industry.

The ESC CPG supervises and coordinates the preparation of new Guidelines. The Committee is also responsible for the endorsement process of these Guidelines. The ESC Guidelines undergo extensive review by the CPG and external experts. After appropriate revisions the Guidelines are approved by all the experts involved in the Task Force. The finalized document is approved by the CPG and EAS for publication in the European Heart Journal and Atherosclerosis Journal. The Guidelines were developed after careful consideration of the scientific and medical knowledge and the evidence available at the time of their dating.

The task of developing ESC/EAS Guidelines also includes the creation of educational tools and implementation programmes for the recommendations including condensed pocket guideline versions, summary slides, booklets with essential messages, summary cards for non-specialists and an electronic version for digital applications (smartphones, etc.). These versions are abridged and thus, for more detailed information, the user should always access the full text version of the Guidelines, which is freely available via the ESC and EAS websites and hosted on their journals' websites (EHJ and Atherosclerosis Journal). The National Cardiac Societies of the ESC are encouraged to endorse, translate and implement all ESC Guidelines. Implementation programmes are needed because it has been shown that the outcome of disease may be favourably influenced by the thorough application of clinical recommendations.

Health professionals are encouraged to take the ESC/EAS Guidelines fully into account when exercising their clinical judgment, as well as in the determination and the implementation of preventive, diagnostic or therapeutic medical strategies. However, the ESC/EAS Guidelines do not override in any way whatsoever the individual responsibility of 


\section{Abbreviations and acronyms}

ABI Ankle-brachial index

ACCELERATE Assessment of Clinical Effects of Cholesteryl Ester Transfer Protein Inhibition with Evacetrapib in Patients at a High-Risk for Vascular Outcomes

ACCORD Action to Control Cardiovascular Risk in Diabetes

ACS Acute coronary syndrome

ALT Alanine aminotransferase

ANGPTL3 Angiopoietin-like protein 3

Apo Apolipoprotein

ART Antiretroviral treatment

ASCEND A Study of Cardiovascular Events iN Diabetes

ASCOT-LLA Anglo-Scandinavian Cardiac Outcomes Trial - LipidLowering Arm

ASCVD Atherosclerotic cardiovascular disease

ASSIGN CV risk estimation model from the Scottish Intercollegiate Guidelines Network

AURORA A study to evaluate the Use of Rosuvastatin in subjects On Regular haemodialysis: an Assessment of survival and cardiovascular events

b.i.d. Twice a day (bis in die)

BIOSTAT-CHF BIOlogy Study to TAilored Treatment in Chronic Heart Failure

BIP Bezafibrate Infarction Prevention

BMI Body mass index

BP Blood pressure

CABG Coronary artery bypass graft surgery

CAC Coronary artery calcium

CAD Coronary artery disease

CANTOS Canakinumab Antiinflammatory Thrombosis Outcome Study

CETP Cholesteryl ester transfer protein

CHD Coronary heart disease

CI Confidence interval

CIID Chronic immune-mediated inflammatory diseases

CIRT Cardiovascular Inflammation Reduction Trial

CK Creatine kinase

CKD Chronic kidney disease

COM-B Capability, Opportunity and Motivation

CORONA Controlled Rosuvastatin Multinational Trial in Heart Failure

CPG Committee for Practice Guidelines

CT Computed tomography

CTT Cholesterol Treatment Trialists

CV Cardiovascular

CVD Cardiovascular disease

CYP Cytochrome P450

4D Die Deutsche Diabetes Dialyse Studie

dal-OUTCOMES Effects of Dalcetrapib in Patients with a Recent Acute Coronary Syndrome

DASH Dietary Approaches to Stop Hypertension

DGAT-2 Diacylglycerol acyltransferase-2

DHA Docosahexaenoic acid

DM Diabetes mellitus

EAPC European Association of Preventive Cardiology

EAS European Atherosclerosis Society

EBBIGHAUS Evaluating PCSK9 Binding Antibody Influence on Cognitive Health in High Cardiovascular Risk Subjects

eGFR Estimated glomerular filtration rate

EMA European Medicines Agency

EPA Eicosapentaenoic acid

ESC European Society of Cardiology

EVOLVE EpanoVa fOr Lowering Very high triglyceridEs

EVOPACS EVOlocumab for early reduction of LDL-cholesterol levels in patients with Acute Coronary Syndromes

FCH Familial combined hyperlipidaemia

FCS Familial chylomicronaemia syndrome

FDA US Food and Drug Administration

FH Familial hypercholesterolaemia

FIELD Fenofibrate Intervention and Event Lowering in Diabetes

FOCUS Fixed-Dose Combination Drug for Secondary Cardiovascular Prevention

FOURIER Further Cardiovascular Outcomes Research with PCSK9 Inhibition in Subjects with Elevated Risk

GFR Glomerular filtration rate

GI Gastrointestinal

GISSI Gruppo Italiano per lo Studio della Sopravvivenza nell'Infarto Miocardico

HbA1c Glycated haemoglobin

HeFH Heterozygous familial hypercholesterolaemia

HDL High-density lipoprotein

HDL-C High-density lipoprotein cholesterol

HF Heart failure

HHS Helsinki Heart Study

HIV Human immunodeficiency virus

HMG-CoAHydroxymethylglutaryl-coenzyme A

$\mathrm{HoFH}$ Homozygous familial hypercholesterolaemia

HPS2-THRIVE Heart Protection Study 2-Treatment of HDL to

Reduce the Incidence of Vascular Events

HR Hazard ratio

HTG Hypertriglyceridaemia

IDEA L Incremental Decrease In End-points Through Aggressive Lipid-lowering

IDL Intermediate-density lipoproteins

IL Interleukin

ILLUMINATE Investigation of Lipid Level Management to Understand its Impact in Atherosclerotic Events

IMPROVE-IT Improved Reduction of Outcomes: Vytorin Efficacy International Trial

IPD Individual participant data

JUPITER Justification for the Use of Statins in Prevention: an Intervention Trial Evaluating Rosuvastatin

KDIGO Kidney Disease: Improving Global Outcomes

LCAT Lecithin cholesterol acyltransferase

LDL Low-density lipoprotein

LDL-C Low-density lipoprotein cholesterol

LDLR Low-density lipoprotein receptor

LEAD Lower extremity arterial disease

LEADER Lower Extremity Arterial Disease Event Reduction

LPL Lipoprotein lipase

Lp(a) Lipoprotein(a)

mAb Monoclonal antibody

MACE Major adverse cardiovascular events

MESA Multi-Ethnic Study of Atherosclerosis

MetS Metabolic syndrome

MI Myocardial infarction

mRNA Messenger RNA

MTP Microsomal triglyceride transfer protein

NAFLD Non-alcoholic fatty liver disease

NNT Number needed to treat

NPC1L1 Niemann-Pick C1-like protein 1

NSTE-ACS Non-ST elevation acute coronary syndrome

o.d. Once a day (omni die)

ODYSSEY Outcomes Evaluation of Cardiovascular Outcomes After an Acute Coronary Syndrome During Treatment With Alirocumab

PAD Peripheral arterial disease

PCI Percutaneous coronary intervention

PCSK9 Proprotein convertase subtilisin/kexin type 9 
PPAR- $\alpha$ Peroxisome proliferator-activated receptor- $\alpha$ PREDIMED Prevención con Dieta Mediterránea

PROCAM Prospective Cardiovascular Munster Study

PROMINENT Pemafibrate to Reduce Cardiovascular OutcoMes by Reducing Triglycerides IN PatiENts With DiabeTes

PUFA Polyunsaturated fatty acid

PURE Prospective Urban Rural Epidemiology

RA Rheumatoid arthritis

RCT Randomized controlled trial

REDUCE-IT Reduction of Cardiovascular Events with EPAIntervention Trial

REVEAL Randomized EValuation of the Effects of Anacetrapib Through Lipid modification

RR Relative risk

RYR Red yeast rice

SAMS Statin-associated muscle symptoms

SBP Systolic blood pressure

SCORE Systematic Coronary Risk Estimation

SEAS Simvastatin and Ezetimibe in Aortic Stenosis

SECURE-PCI Statins Evaluation in Coronary Procedures and Revascularization

SFA Saturated fatty acid

SHARP Study of Heart and Renal Protection

siRNA Small interfering RNA
SMI Severe mental illness

SPARCL Stroke Prevention by Aggressive Reduction in Cholesterol Levels

STAREE STAtin Therapy for Reducing Events in the Elderly

STEMI ST-elevation myocardial infarction

STRENGTH Outcomes Study to Assess STatin Residual Risk Reduction with EpaNova in HiGh CV Risk PatienTs with Hypertriglyceridemia

TC Total cholesterol

T1DM Type 1 diabetes mellitus

T2DM Type 2 diabetes mellitus

TGs Triglycerides

TIA Transient ischaemic attack

TIMI Thrombolysis In Myocardial Infarction

TNF Tumour necrosis factor

TNT Treating to New Targets

TRL Triglyceride-rich lipoprotein

ULN Upper limit of normal

VA-HIT Veterans Affairs High Density Lipoprotein Intervention Trial

VITAL VITamin D and OmegA-3 Trial

VLDL Very low-density lipoprotein

WHO World Health Organization

WOSCOPS West of Scotland Coronary Prevention Study health professionals to make appropriate and accurate decisions in consideration of each patient's health condition and in consultation with that patient or the patient's caregiver where appropriate and/or necessary. It is also the health professional's responsibility to verify the rules and regulations applicable in each country to drugs and devices at the time of prescription.

\section{Introduction}

The previous ESC/EAS lipid Guidelines were published in August 2016 [1]. The emergence of a substantial body of evidence over the last few years has required new, up-to-date Guidelines.

New evidence has confirmed that the key initiating event in atherogenesis is the retention of low-density lipoprotein (LDL) cholesterol (LDL-C) and other cholesterol-rich apolipoprotein (Apo) B-containing lipoproteins within the arterial wall [2]. Several recent placebo-controlled clinical studies have shown that the addition of either ezetimibe or antiproprotein convertase subtilisin/kexin type 9 (PCSK9) monoclonal antibodies (mAbs) to statin therapy provides a further reduction in atherosclerotic cardiovascular disease (ASCVD) risk, which is directly and positively correlated with the incrementally achieved absolute LDL-C reduction. Furthermore, these clinical trials have clearly indicated that the lower the achieved LDL-C values, the lower the risk of future cardiovascular (CV) events, with no lower limit for LDL-C values, or ' $J$ '-curve effect. In addition, studies of the clinical safety of these very low achieved LDL-C values have proved reassuring, albeit monitoring for longer periods is required. For raising high-density lipoprotein (HDL) cholesterol (HDLC), recent studies have indicated that the currently available therapies do not reduce the risk of ASCVD. Finally, human Mendelian randomization studies have demonstrated the critical role of LDL-C, and other cholesterol-rich ApoB-containing lipoproteins, in atherosclerotic plaque formation and related subsequent CV events. Thus, there is no longer an 'LDL-C hypothesis', but established facts that increased LDL-C values are causally related to ASCVD, and that lowering LDL particles and other ApoB-containing lipoproteins as much as possible reduces $\mathrm{CV}$ events.

In order to be aligned with these new findings, the ESC/EAS Task Force members who have written these Guidelines have proposed new LDL-C goals, as well as a revised CV risk stratification, which are especially relevant to high- and very-high-risk patients.
These novel ESC/EAS Guidelines on lipids provide important new advice on patient management, which should enable more clinicians to efficiently and safely reduce CV risk through lipid modification (see Table 2).

\subsection{What is new in the 2019 guidelines?}

New recommendations, and new and revised concepts, are presented in Table 3.

\section{What is cardiovascular disease prevention?}

\subsection{Definition and rationale}

Cardiovascular disease (CVD), of which ASCVD is the major component, is responsible for $>4$ million deaths in Europe each year. It kills more women ( 2.2 million) than men ( 1.8 million), although $\mathrm{CV}$ deaths before the age of 65 years are more common in men (490 000 vs. 193000 ) [3]. Prevention is defined as a co-ordinated set of actions, either at the population or individual level, aimed at eradicating and eliminating are synonymous or minimizing the impact of CV diseases and their related disabilities. More patients are surviving their first CVD event and are at high-risk of recurrences. In addition, the prevalence of some risk factors, notably diabetes (DM) and obesity, is increasing. The importance of ASCVD prevention remains undisputed and should be delivered at the general population level by promoting healthy lifestyle behaviour [4], and at the individual level by tackling unhealthy lifestyles and by reducing increased levels of causal CV risk factors, such as LDL cholesterol or blood pressure (BP) levels.

\subsection{Development of the joint Task Force guidelines for the management of dyslipidaemias}

The present Guidelines represent an evidence-based consensus of the European Task Force, including the ESC and the EAS.

By appraising the current evidence and identifying remaining knowledge gaps in the management of dyslipidaemias, the Task Force has formulated recommendations to guide action in clinical practice to prevent ASCVD by modifying plasma lipid levels. 
Table 1

Classes of recommendations.

\begin{tabular}{|c|c|c|}
\hline \multicolumn{2}{|r|}{ Definition } & Wording to use \\
\hline Class I & $\begin{array}{l}\text { Evidence and/or general agreement } \\
\text { that a given treatment or procedure is } \\
\text { beneficial, useful, effective. }\end{array}$ & Is recommended or is indicated \\
\hline Class II & \multicolumn{2}{|c|}{$\begin{array}{l}\text { Conflicting evidence and/or a divergence of opinion about the usefulness/ } \\
\text { efficacy of the given treatment or procedure. }\end{array}$} \\
\hline Class Ila & $\begin{array}{l}\text { Weight of evidence/opinion is in } \\
\text { favour of usefulness/efficacy. }\end{array}$ & Should be considered \\
\hline Class IIb & $\begin{array}{l}\text { Usefulness/efficacy is less well } \\
\text { established by evidence/opinion. }\end{array}$ & May be considered \\
\hline Class III & $\begin{array}{l}\text { Evidence or general agreement that the } \\
\text { given treatment or procedure is not } \\
\text { useful/effective, and in some cases } \\
\text { may be harmful. }\end{array}$ & Is not recommended \\
\hline
\end{tabular}

This document has been developed for healthcare professionals to facilitate informed communication with individuals about their CV risk and the benefits of adopting and sustaining a healthy lifestyle, and of early modification of their lipid-related CV risk. In addition, the Guidelines provide tools for healthcare professionals to promote up-todate intervention strategies, integrate these strategies into national or regional prevention frameworks, and to translate them into locally delivered healthcare services, in line with the recommendations of the World Health Organization (WHO) Global Status Report on Noncommunicable Diseases 2014 [5].

A lifetime approach to CV risk should be considered [1]. This implies that-apart from improving lifestyle habits and reducing risk factor levels in patients with established ASCVD, and in those at increased risk of developing ASCVD-people of all ages should be encouraged to adopt or sustain a healthy lifestyle.

\section{Total cardiovascular risk}

\subsection{Total cardiovascular risk estimation}

$\mathrm{CV}$ risk in the context of these Guidelines means the likelihood of a person developing an atherosclerotic $\mathrm{CV}$ event over a defined period of time. Total CVD risk expresses the combined effect of a number of risk factors on this risk estimate. In these Guidelines, we address the lipid-related contribution to total $\mathrm{CV}$ risk and how to manage it at the clinical level.

\subsubsection{Rationale for assessing total cardiovascular disease risk}

All current guidelines on the prevention of ASCVD in clinical practice recommend the assessment of total CVD risk. Prevention of ASCVD in a given person should relate to his or her total CV risk: the higher the risk, the more intense the action should be.

Many risk assessment systems are available and have been comprehensively reviewed (Supplementary Table 1 in the Supplementary Data). Most guidelines use one of these risk assessment systems [6-8]. Ideally, risk charts should be based on country-specific cohort data. These are not available for most countries. The SCORE (Systematic Coronary Risk Estimation) system can be recalibrated for use in different populations by adjusting for secular changes in CVD mortality and risk factor prevalence. Calibrated country-specific versions are available for many European countries and can be found at http:// www.heartscore.org. These are now being updated to provide recalibrated, contemporaneous country-specific charts for all European

Table 2

Levels of evidence.
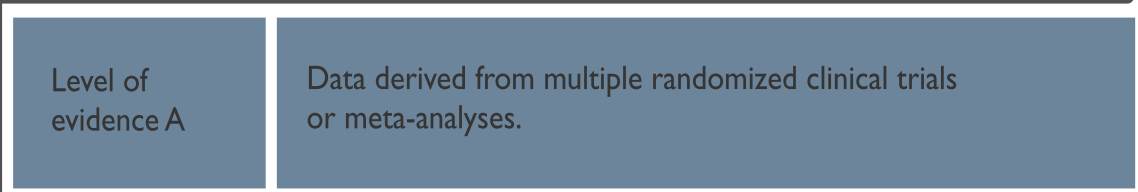

Level of

evidence B

Data derived from a single randomized clinical trial

or large non-randomized studies.

Level of

evidence C

Consensus of opinion of the experts and/or small studies, retrospective studies, registries. 
Table 3

New recommendations, and new and revised concepts.

\section{New recommendations}

\section{Cardiovascular imaging for assessment of ASCVD risk}

Assessment of arterial (carotid and/or femoral) plaque burden on arterial ultrasonography should be considered as a risk modifier in individuals at low or moderate risk.

\section{Cardiovascular imaging for assessment of ASCVD risk}

CAC score assessment with CT should be considered as a risk modifier in the $\mathrm{CV}$ risk assessment of asymptomatic individuals at low or moderate risk.

\section{Lipid analyses for CVD risk estimation}

$\mathrm{Lp}(\mathrm{a})$ measurement should be considered at least once in each adult person's lifetime to identify those with very high inherited Lp(a) levels $>180 \mathrm{mg} / \mathrm{dL}$ ( $>430 \mathrm{nmol} / \mathrm{L})$ who may have a lifetime risk of ASCVD equivalent to the risk associated with heterozygous familial hypercholesterolaemia.

\section{Drug treatments of patients with hypertriglyceridaemia}

In high-risk (or above) patients with TG between 1.5 and $5.6 \mathrm{mmol} / \mathrm{L}(135-499 \mathrm{mg} / \mathrm{dL})$ despite statin treatment, $\mathrm{n}-3$ PUFAs

(icosapent ethyl $2 \times 2 \mathrm{~g} /$ day) should be considered in combination with statins.

\section{Treatment of patients with heterozygous $\mathrm{FH}$}

In primary prevention, for individuals with $\mathrm{FH}$ at very-high risk, an LDL-C reduction of $\geq 50 \%$ from baseline and an LDL-C goal of $<1.4 \mathrm{mmol} / \mathrm{L}$ $(<55 \mathrm{mg} / \mathrm{dL})$ should be considered.

\section{Treatment of dyslipidaemias in older people}

Treatment with statins is recommended for primary prevention, according to the level of risk, in older people aged $\leq 75$.

Treatment of dyslipidaemias in older people Initiation of statin treatment for primary prevention in older people aged $>75$ may be considered, if at high risk or above.

\section{Treatment of dyslipidaemias in DM}

In patients with T2DM at very-high risk, an LDL-C reduction of $\geq 50 \%$ from baseline and an LDL-C goal of $<1.4 \mathrm{mmol} / \mathrm{L}(<55 \mathrm{mg} / \mathrm{dL})$ is recommended. In patients with T2DM at high risk, an LDL-C reduction of $\geq 50 \%$ from baseline and an LDL-C goal of $<1.8 \mathrm{mmol} / \mathrm{L}(<70 \mathrm{mg} / \mathrm{dL})$ is recommended. Statins are recommended in patients with T1DM who are at high or very-high risk.

\section{Treatment of dyslipidaemias in DM}

Intensification of statin therapy should be considered before the introduction of combination therapy.

If the goal is not reached, statin combination with ezetimibe should be considered.

\section{Treatment of dyslipidaemias in DM}

Statin therapy is not recommended in pre-menopausal patients with DM who are considering pregnancy or not using adequate contraception.

\section{Lipid-lowering therapy in patients with ACS}

For patients who present with an ACS, and whose LDL-C levels are not at goal despite already taking a maximally tolerated statin dose and ezetimibe, adding a PCSK9 inhibitor early after the event (if possible, during hospitalization for the ACS event) should be considered.

\section{Changes in recommendations}

\section{Upgrades}

2016

Lipid analyses for CVD risk estimation

ApoB should be considered as an alternative risk marker whenever available, especially in individuals with high TG.

\section{9}

\section{Lipid analyses for CVD risk estimation}

ApoB analysis is recommended for risk assessment, particularly in people with high TG, DM, obesity or metabolic syndrome, or very low LDL-C. It can be used as an alternative to LDL-C, if available, as the primary measurement for screening, diagnosis, and management, and may be preferred over non-HDL-C in people with high TG, DM, obesity, or very low LDL-C.

\section{Pharmacological LDL-C lowering}

If the goals are not achieved with the maximum tolerated dose of statin, combination with ezetimibe is recommended.

If the LDL goal is not reached, statin combint
absorption inhibitor should be considered.

\section{Pharmacological LDL-C lowering}

In patients at very-high risk, with persistent high LDL-C despite treatment with maximal tolerated statin dose, in combination with ezetimibe or in patients with statin intolerance, a PCSK9 inhibitor may be considered.

\section{Pharmacological LDL-C lowering}

For secondary prevention, patients at very-high risk not achieving their goal on a maximum tolerated dose of statin and ezetimibe, a combination with a PCSK9 inhibitor is recommended.

For very-high-risk FH patients (that is, with ASCVD or with another major risk factor) who do not achieve their goals on a maximum tolerated dose of statin and ezetimibe, a combination with a PCSK9 inhibitor is recommended. 
Drug treatments of hypertriglyceridaemia

Statin treatment may be considered as the first drug of choice for

reducing CVD risk in high-risk individuals with hypertriglyceridaemia.

\section{Treatment of patients with heterozygous FH}

Treatment should be considered to aim at reaching an LDL-C $<2.6 \mathrm{mmol} / \mathrm{L}$ $(<100 \mathrm{mg} / \mathrm{dL})$ or in the presence of CVD $<1.8 \mathrm{mmol} / \mathrm{L}(<70 \mathrm{mg} / \mathrm{dL})$. If targets cannot be reached, maximal reduction of LDL-C should be considered using appropriate drug combinations.

\section{Treatment of patients with heterozygous FH}

Treatment with a PCSK9 antibody should be considered in $\mathrm{FH}$ patients with CVD or with other factors putting them at very-high risk for $\mathrm{CHD}$, such as other $\mathrm{CV}$ risk factors, family history, high $\mathrm{Lp}(\mathrm{a})$, or statin intolerance.

\section{Treatment of dyslipidaemias in older adults}

Since older people often have comorbidities and have altered pharmacokinetics, lipid-lowering medication should be started at a lower dose and then titrated with caution to achieve target lipid levels that are the same as in younger people.

\section{Lipid-lowering therapy in patients with ACS}

If the LDL-C target is not reached with the highest tolerated statin dose and/or ezetimibe, PCSK9 inhibitors may be considered on top of lipid-lowering therapy; or alone or in combination with ezetimibe in statin-intolerant patients or in whom a statin is contraindicated.

\section{Recommendation grading}

Statin treatment is recommended as the first drug of choice for reducing CVD risk in high-risk individuals with hypertriglyceridaemia [TG $>2.3 \mathrm{mmol} / \mathrm{L}(200 \mathrm{mg} / \mathrm{dL})]$.

Treatment of patients with heterozygous $\mathrm{FH}$

\section{For FH patients with ASCVD who are at very-high risk, treatment to} achieve at least a $50 \%$ reduction from baseline and an LDL-C $<1.4 \mathrm{mmol} / \mathrm{L}(<55 \mathrm{mg} / \mathrm{dL}$ ) is recommended. If goals cannot be achieved, a drug combination is recommended.

\section{Treatment of patients with heterozygous FH}

Treatment with a PCSK9 inhibitor is recommended in very-high-risk FH patients if the treatment goal is not achieved on maximal tolerated statin plus ezetimibe.

\section{Treatment of dyslipidaemias in older people}

It is recommended that the statin is started at a low dose if there is significant renal impairment and/or the potential for drug interactions, and then titrated upwards to achieve LDL-C treatment goals.

\section{New sections}

\section{Lipid-lowering therapy in patients with ACS}

If the LDL-C goal is not achieved after 4-6 weeks despite maximal tolerated statin therapy and ezetimibe, addition of a PCSK9 inhibitor is recommended.

- A new section is focused on the utility of non-invasive CV imaging for classification of total CVD risk, with implications for recommended lipid-modifying therapies.

- More data are provided on the biology and physiology of lipids and lipoproteins, and on their roles in pathophysiology. Emerging evidence from observational studies, RCTs, and genetic (Mendelian randomization) studies unequivocally showing a causal effect of LDL-C in the development of ASCVD is discussed, and newer evidence regarding the effects of TGs and HDL on ASCVD risk is presented.

- New sections describe novel lipid-modifying medications as well as emerging approaches for lowering LDL-C, TGs, and Lp(a).

- A new section discusses the inflammation-related risk in very high-risk patients and the potential role of inflammation as a therapeutic target to lower ASCVD risk. - New/revised concepts

More intensive reduction of LDL-C across CV risk categories

- For secondary prevention in very-high-risk patients, an LDL-C reduction of $\geq 50 \%$ from baseline and an LDL-C goal of $<1.4 \mathrm{mmoVL}(<55 \mathrm{mg} / \mathrm{dL})$ are recommended. - For patients with ASCVD who experience a second vascular event within 2 years (not necessarily of the same type as the first event) while taking maximally tolerated statin therapy, an LDL-C goal of $<1.0 \mathrm{mmol} / \mathrm{L}(<40 \mathrm{mg} / \mathrm{dL})$ may be considered.

- In primary prevention, for individuals at very-high risk but without $\mathrm{FH}$, an $\mathrm{LDL}-\mathrm{C}$ reduction of $\geq 50 \%$ from baseline and an $\mathrm{LDL}-\mathrm{C}$ goal of $<1.4 \mathrm{mmol} / \mathrm{L}$ $(<55 \mathrm{mg} / \mathrm{dL})$ are recommended. For individuals at very-high risk (that is, with another risk factor but without ASCVD), in primary prevention the same goals for LDL-C lowering should be considered.

- For patients at high risk, an LDL-C reduction of $\geq 50 \%$ from baseline and an LDL-C goal of $<1.8 \mathrm{mmol} / \mathrm{L}(<70 \mathrm{mg} / \mathrm{dL})$ are recommended.

- For individuals at moderate risk, an LDL-C goal of $<2.6 \mathrm{mmol} / \mathrm{L}(<100 \mathrm{mg} / \mathrm{dL})$ should be considered.

- For individuals at low risk, an LDL-C goal of $<3.0 \mathrm{mmol} / \mathrm{L}(<116 \mathrm{mg} / \mathrm{dL})$ may be considered.

The rationale for the revised, lower LDL-C goals across CV risk categories is discussed, based on a critical synthesis of available evidence

from lipid-modifying interventions resulting in reductions in CV risk.

Pharmacological LDL-C-lowering strategies

The section on pharmacological strategies to lower LDL-C emphasizes the concept that the absolute LDL-C reduction (determined by pre-treatment LDL$\mathrm{C}$ levels and the LDL-lowering efficacy of the medications) dictates the relative risk reduction, which in turn-depending on the baseline $\mathrm{CV}$ risk — defines the associated absolute $\mathrm{CV}$ risk reduction in individual patients.

Risk classification in patients with $\mathrm{FH}$

Patients with FH and ASCVD, or another major risk factor, are classified as very-high-risk, and those without known ASCVD and without other risk factors as high-risk. Recommended treatment goals are defined accordingly.

\section{Adverse effects of statins}

The distinction between formal statin myopathy vs. so-called statin-associated muscle symptoms is emphasized, and the discordance in reported frequency of symptoms in RCTs vs. observational studies are critically discussed on the basis of new relevant evidence.

\section{PCSK9 inhibitors}

New outcome study data of PCSK9 inhibitors are presented, and updated recommendations for their clinical use are provided.

Cost-effectiveness

The issue of cost-effectiveness of lipid-modifying interventions is updated in view of changes in the availability of generic products for statins and ezetimibe, and of PCSK9 inhibitors. 


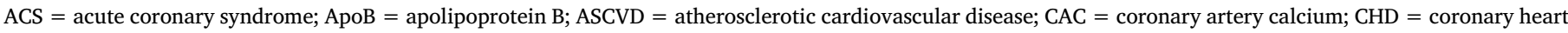

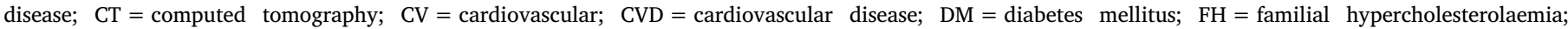

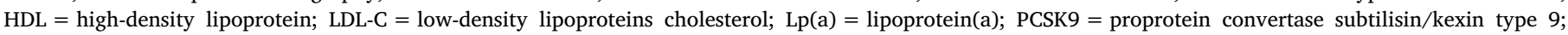

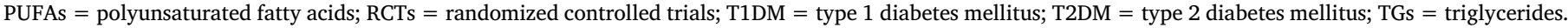

countries. Other risk estimation systems-using both fatal and non-fatal events-can also be recalibrated, but the process is easier and scientifically more robust for mortality than for total events. The European Guidelines on CVD prevention in clinical practice (both the 2012 [9] and 2016 [10] versions) recommend the use of the SCORE system because it is based on large, representative European cohort data sets and because it is relatively straightforward to recalibrate for individual countries.

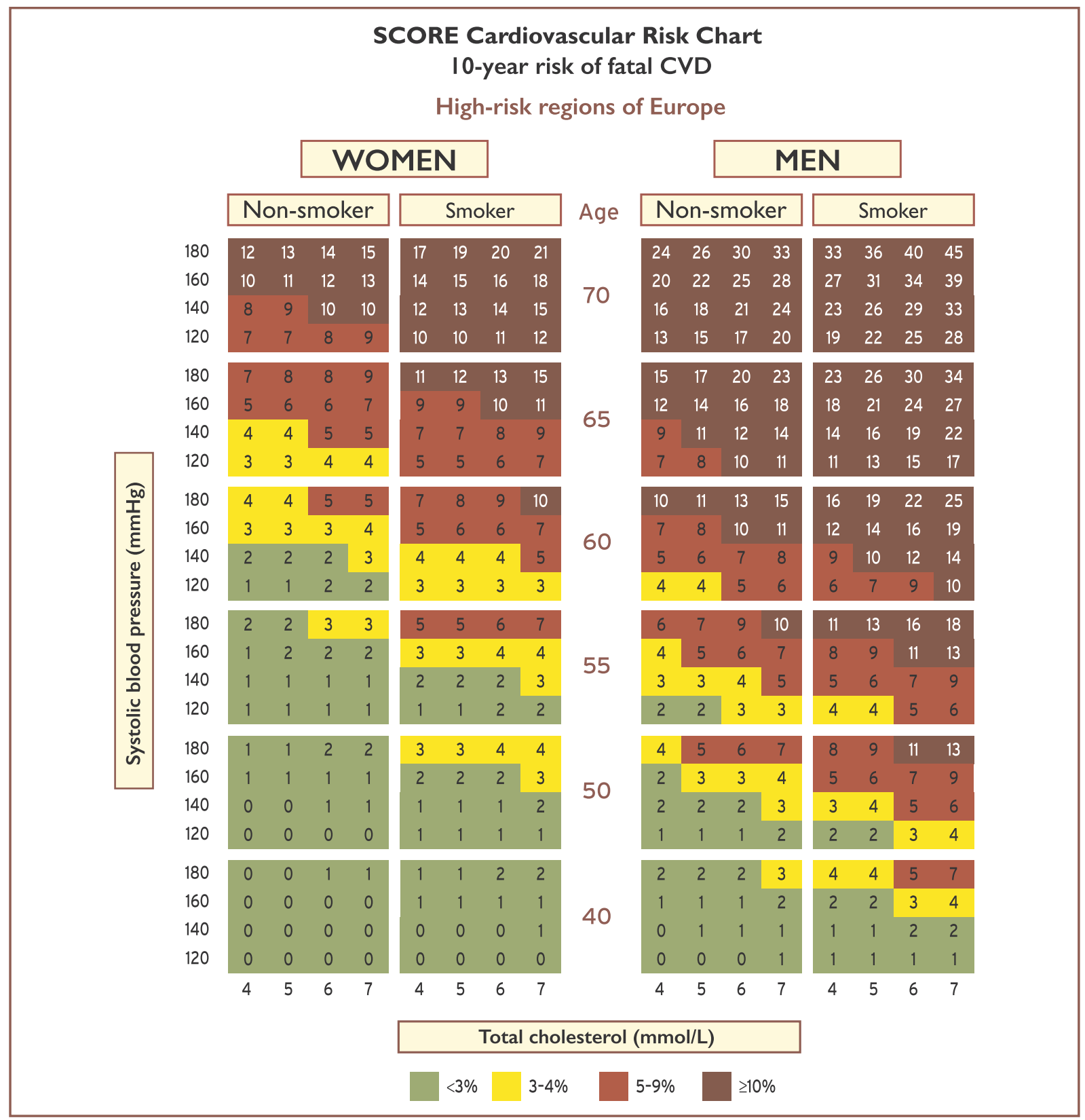

Fig. 1. Systematic Coronary Risk Estimation chart for European populations at high cardiovascular disease risk. The 10-year risk of fatal cardiovascular disease in populations at high cardiovascular disease risk based on the following risk factors: age, gender, smoking, systolic blood pressure, and total cholesterol. To convert the risk of fatal cardiovascular disease to risk of total (fatal + non-fatal) cardiovascular disease, multiply by 3 in men and by 4 in women, and slightly less in older people. Note: the Systematic Coronary Risk Estimation chart is for use in people without overt cardiovascular disease, diabetes (type 1 and 2), chronic kidney disease, familial hypercholesterolaemia, or very high levels of individual risk factors because such people are already at high-risk and need intensive risk factor management. Cholesterol: $1 \mathrm{mmoL} / \mathrm{L}=38.67 \mathrm{mg} / \mathrm{dL}$. The SCORE risk charts presented above differ slightly from those in the 2016 European Society of Cardiology/European Atherosclerosis Society Guidelines for the management of dyslipidaemias and the 2016 European Guidelines on cardiovascular disease prevention in clinical practice, in that: (i) age has been extended from age 65 to 70; (ii) the interaction between age and each of the other risk factors has been incorporated, thus reducing the overestimation of risk in older persons in the original Systematic Coronary Risk Estimation charts; and (iii) the cholesterol band of $8 \mathrm{mmoL} / \mathrm{L}$ has been removed, since such persons will qualify for further evaluation in any event. SCORE $=$ Systematic Coronary Risk Estimation. 


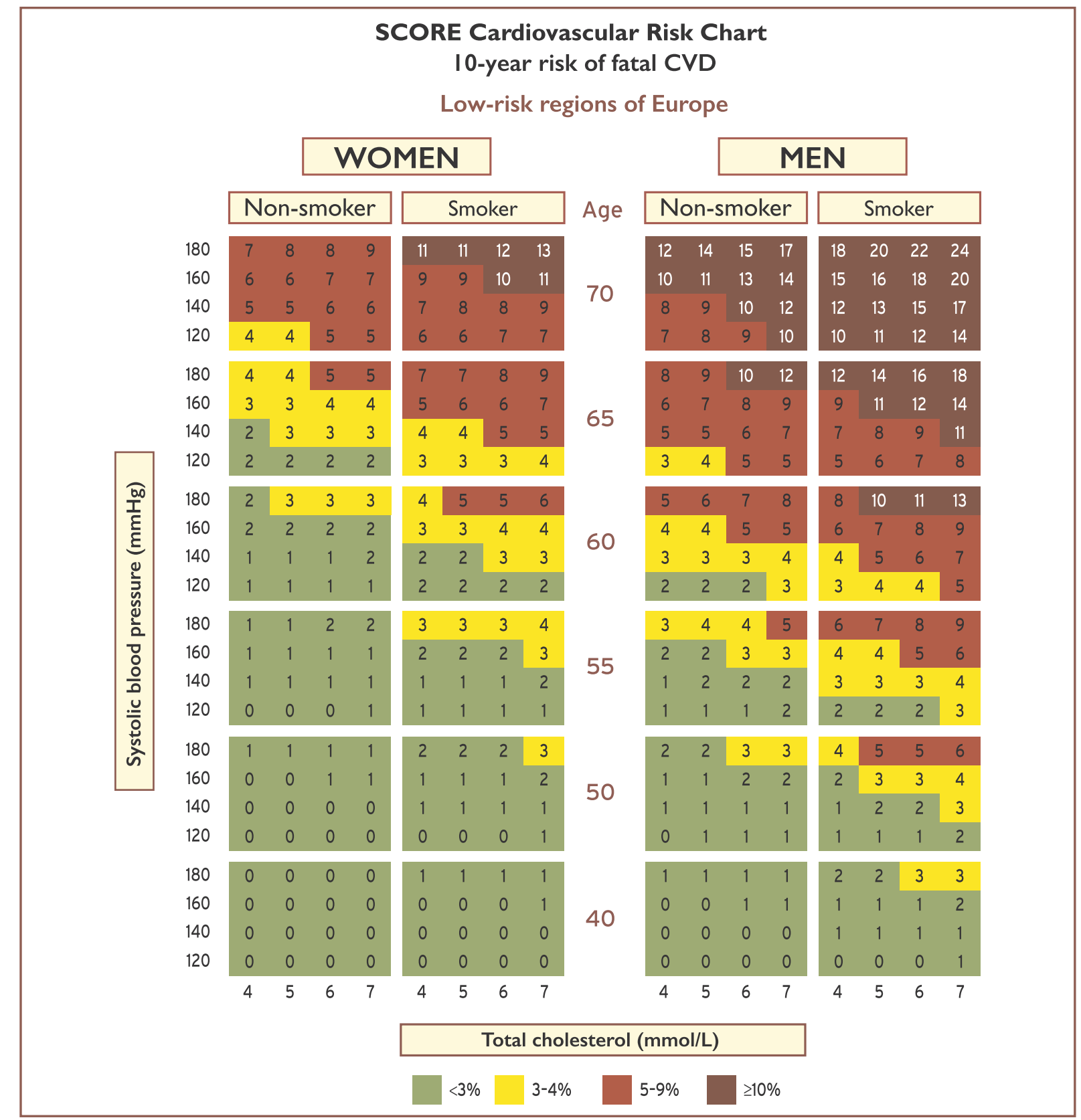

Fig. 2. Systematic Coronary Risk Estimation chart for European populations at low cardiovascular disease risk. The 10-year risk of fatal cardiovascular disease in populations at low cardiovascular disease risk based on the following risk factors: age, gender, smoking, systolic blood pressure, and total cholesterol. To convert the risk of fatal cardiovascular disease to risk of total (fatal + non-fatal) cardiovascular disease, multiply by 3 in men and by 4 in women, and slightly less in older people. Note: the Systematic Coronary Risk Estimation chart is for use in people without overt cardiovascular disease, diabetes (type 1 and 2), chronic kidney disease, familial hypercholesterolaemia, or very high levels of individual risk factors because such people are already at high-risk and need intensive risk factor management. Cholesterol: $1 \mathrm{mmoL} / \mathrm{L}=38.67 \mathrm{mg} / \mathrm{dL}$. The SCORE risk charts presented above differ slightly from those in the 2016 European Society of Cardiology/European Atherosclerosis Society Guidelines for the management of dyslipidaemias and the 2016 European Guidelines on cardiovascular disease prevention in clinical practice, in that: (i) age has been extended from age 65 to 70; (ii) the interaction between age and each of the other risk factors has been incorporated, thus reducing the overestimation of risk in older persons in the original Systematic Coronary Risk Estimation charts; and (iii) the cholesterol band of $8 \mathrm{mmoL} / \mathrm{L}$ has been removed since such persons will qualify for further evaluation in any event. SCORE = Systematic Coronary Risk Estimation.

Persons with documented ASCVD, type 1 or type 2 DM (T1DM and T2DM, respectively), very high levels of individual risk factors, or chronic kidney disease (CKD) are generally at very-high or high total $\mathrm{CV}$ risk. No risk estimation models are needed for such persons; they all need active management of all risk factors. For other, apparently healthy people, the use of a risk estimation system such as SCORE, which estimates the 10 year cumulative risk of a first fatal atherosclerotic event, is recommended to estimate total CV risk, since many people have several risk factors that, in combination, may result in high levels of total CV risk.

Risk estimates have been produced as charts for high- and low-risk regions in Europe (Fig. 1 and 2) [11]. All International Classification of Diseases codes that are related to deaths from vascular origin caused by atherosclerosis are included. The reasons for retaining a system that estimates fatal as opposed to total fatal + non-fatal events are that nonfatal events are dependent on definition, developments in diagnostic tests, and methods of ascertainment, all of which can vary, resulting in very variable multipliers to convert fatal to total events. In addition, 
Box 1

How to use the risk estimation charts

To estimate a person's 10-year risk of CVD death, find the table for his/her gender, smoking status, and age. Within the table, find the cell nearest to the person's BP and TC. Risk estimates will need to be adjusted upwards as the person approaches the next age category.

Risk is initially assessed on the level of TC and systolic BP before treatment, if known. The longer the treatment and the more effective it is, the greater the reduction in risk, but in general it will not be more than about one-third of the baseline risk. For example, for a person on antihypertensive drug treatment in whom the pre-treatment BP is not known, if the total CV SCORE risk is 6\%, then the pre-treatment total CV risk may have been $9 \%$.

Low-risk persons should be offered advice to maintain their low-risk status. While no threshold is universally applicable, the intensity of advice should increase with increasing risk.

The charts may be used to give some indication of the effects of reducing risk factors, given that there is apparently a time lag before the risk reduces. In general, people who stop smoking halve their cumulative risk over a relatively short period of time.

$\mathrm{BP}=$ blood pressure; $\mathrm{CV}=$ cardiovascular; $\mathrm{CVD}=$ cardiovascular disease; SCORE $=$ Systematic Coronary Risk Estimation; $\mathrm{TC}=$ total cholesterol.

Box 2

Risk estimation charts for different countries

The low-risk charts should be considered for use in Austria, Belgium, Cyprus, Denmark, Finland, France, Germany, Greece, Iceland, Ireland, Israel, Italy, Luxembourg, Netherlands, Norway, Malta, Portugal, Slovenia, Spain, Sweden, Switzerland, and the UK.

The high-risk charts should be considered for use in Albania, Algeria, Armenia, Bosnia and Herzegovina, Croatia, Czech Republic, Estonia, Hungary, Latvia, Lebanon, Libya, Lithuania, Montenegro, Morocco, Poland, Romania, Serbia, Slovakia, Tunisia, and Turkey.

Some countries have a cardiovascular disease mortality rate $>350 / 100000$, and the high-risk chart may underestimate risk. These are Azerbaijan, Belarus, Bulgaria, Egypt, Georgia, Kazakhstan, Kyrgyzstan, Macedonia, Republic of Moldova, Russian Federation, Syria, Tajikistan, Turkmenistan, Ukraine, and Uzbekistan.

See http://apps.who.int/gho/data/node.home.

Box 3

Qualifiers

The charts can assist in risk assessment and management, but must be interpreted in light of the clinician's knowledge and experience, and of the patient's pre-test likelihood of CVD.

Risk will be overestimated in countries with decreasing CVD mortality, and underestimated in countries in which mortality is increasing.

This is dealt with by recalibration (http://www.heartscore.org/en_GB/).

Risk estimates are lower in women than in men. However, risk is only deferred in women; the risk of a 60-year-old woman is similar to that of a 50-year-old man. Ultimately, more women die from CVD than men.

Relative risks may be unexpectedly high in young persons, even if absolute risk levels are low. The relative risk chart (Supplementary Fig. 1) and the estimated risk age (Supplementary Fig. 2) may be helpful in identifying and counselling such persons.

$\mathrm{CVD}=$ cardiovascular disease

Box 4

Factors modifying Systematic Coronary Risk Estimation risks

Social deprivation: the origin of many of the causes of CVD.

Obesity and central obesity as measured by the body mass index and waist circumference, respectively.

Physical inactivity.

Psychosocial stress including vital exhaustion.

Family history of premature CVD (men: $<55$ years and women: $<60$ years).

Chronic immune-mediated inflammatory disorder.

Major psychiatric disorders.

Treatment for human immunodeficiency virus infection.

Atrial fibrillation.

Left ventricular hypertrophy.

Chronic kidney disease.

Obstructive sleep apnoea syndrome.

Non-alcoholic fatty liver disease.

$\mathrm{CVD}=$ cardiovascular disease

total event charts, in contrast to those based on mortality, are more difficult to recalibrate to suit different populations. That said, work is in progress to produce regional total event charts .

The SCORE data indicate that the total CVD event risk is about three times higher than the risk of fatal CVD for men, so a SCORE risk of $5 \%$ translates into a CVD risk of $\sim 15 \%$ of total (fatal + non-fatal) CVD endpoints; the multiplier is higher in women and lower in older people.

Clinicians often ask for thresholds to trigger certain interventions. This is problematic since risk is a continuum and there is no threshold at which, for example, a drug is automatically indicated. This is true for 
Box 5

Risk estimation: key messages

In apparently healthy persons, CVD risk is most frequently the result of multiple, interacting risk factors. This is the basis for total CV risk estimation and management.

Risk factor screening including the lipid profile should be considered in men $>40$ years old, and in women $>50$ years of age or postmenopausal.

A risk estimation system such as SCORE can assist in making logical management decisions, and may help to avoid both under- and overtreatment.

Certain individuals declare themselves to be at high or very high CVD risk without needing risk scoring, and all risk factors require immediate attention. This is true for patients with documented CVD, older individuals with long-standing DM, familial hypercholesterolaemia, chronic kidney disease, carotid or femoral plaques, coronary artery calcium score $>100$, or extreme Lp(a) elevation.

All risk estimation systems are relatively crude and require attention to qualifying statements.

Additional factors affecting risk can be accommodated in electronic risk estimation systems such as HeartScore (www.heartscore.org).

The total risk approach allows flexibility; if optimal control cannot be achieved with one risk factor, trying harder with the other factors can still reduce risk.

$\mathrm{CV}=$ cardiovascular; CVD = cardiovascular disease; DM = diabetes mellitus; SCORE = Systematic Coronary Risk Estimation

all continuous risk factors such as plasma cholesterol or systolic BP (SBP). Therefore, the goals that are proposed in this document reflect this concept.

A particular problem relates to young people with high levels of risk

Table 4

Cardiovascular risk categories.

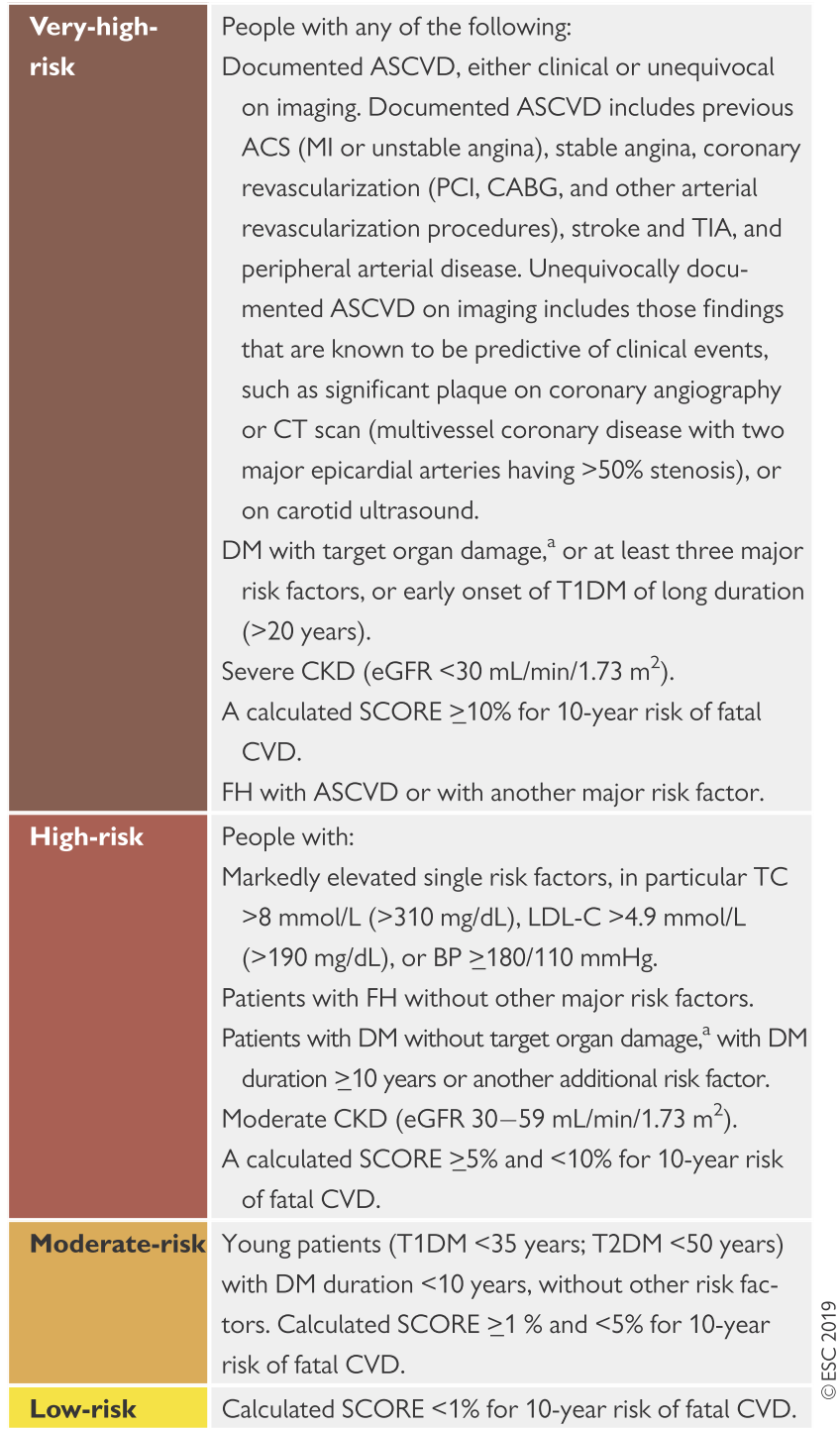

factors; a low absolute risk may conceal a very high relative risk requiring at least intensive lifestyle advice. To motivate young people (i.e. aged $<40$ years) not to delay changing their unhealthy lifestyle, an estimate of their relative risk-illustrating that lifestyle changes can reduce relative risk substantially-may be helpful (Supplementary Fig. 1).

Another approach to this problem is to use CV risk age. The risk age of a person with several CV risk factors is the age of a person with the same level of risk but with ideal levels of risk factors. Thus, a high-risk 40 -year-old would have a risk age $\geq 65$ years. Risk age can be estimated visually by looking at the SCORE chart (as illustrated in Supplementary Fig. 2). In this chart, the risk age of a person with risk factors is defined as the age at which a person with ideal risk factor levels would reach the same risk level. Ideal risk factors are non-smoking, total cholesterol (TC) $\leq 4 \mathrm{mmoL} / \mathrm{L}$ ( $\leq 155 \mathrm{mg} / \mathrm{dL}$ ), and SBP $\leq 120 \mathrm{mmHg}$. Risk age is also automatically calculated as part of the latest revision of HeartScore (http://www.HeartScore.org).

Risk age has been shown to be independent of the CV endpoint used $[6,8]$, can be used in any population regardless of baseline risk or secular changes in mortality, and therefore avoids the need for recalibration.

Lifetime risk is another approach to illustrate the impact of risk factors that may be useful in younger people [12]. The greater the burden of risk factors, the higher the lifetime risk. This approach produces higher risk figures for younger people because of their longer exposure times. Therefore, it is more useful as a way of illustrating risk than as a guide to treatment, because therapeutic trials have been based on a fixed follow-up period and not on lifetime risk.

Another problem relates to older people. In some age categories, the majority of people, especially males, will have estimated 10 year cumulative CV death risks exceeding the $5-10 \%$ level, based on age only, even when other CV risk factor levels are relatively low. Therefore, before initiating treatment in the elderly, clinicians should evaluate patients carefully. The relative strengths of risk factors vary with age and SCORE overestimates risk in older people (that is, those aged $>65$ years) [11]. These Guidelines include illustrative charts for older people (see Figs. 1 and 2). While older people benefit from smoking cessation, and control of hypertension and hyperlipidaemia (see section 9.3), clinical judgement is required to avoid side effects from overmedication.

The additional impact of HDL-C on risk estimation is illustrated in Supplementary Figs. 3 and 4; HDL-C can be used to increase the accuracy of the risk evaluation. In these charts, HDL-C is used categorically. The electronic version of SCORE, HeartScore (http://www. heartscore.org/en_GB/), has been modified to take HDL-C into consideration as a continuous variable. Clinicians should be aware that at extremely high values [above $\sim 2.3 \mathrm{mmoL} / \mathrm{L}(90 \mathrm{mg} / \mathrm{dL})$ ] of HDL-C there appears to be an increased risk of ASCVD, so at such levels HDL-C cannot be used as a risk predictor. 


\subsubsection{How to use the risk estimation charts}

Use of the low- or the high-risk SCORE charts will depend on the CVD mortality experience in each country. While any cut-off point is arbitrary and open to debate, in these Guidelines, the cut-off point for calling a country 'low CVD risk' is based on WHO data derived from the Global Burden of Disease Study.

Countries are categorized as low-risk if their age-adjusted 2016 CVD mortality rate was $<150 / 100000$ (for men and women together) (http://www.who.int/healthinfo/global_burden_disease/estimates/en/ ). Countries with a CVD mortality rate of $\geq 150 / 100000$ or more are considered to be at high-risk.

Boxes 1 to 5 summarize the main points regarding the risk estimation charts and their use.

Social deprivation and psychosocial stress set the scene for increased risk [13]. For those at moderate risk, other factors-including metabolic factors such as increased ApoB, lipoprotein(a) [Lp(a)], triglycerides (TGs), or C-reactive protein; the presence of albuminuria; the presence of atherosclerotic plaque in the carotid or femoral arteries; or the coronary artery calcium (CAC) score-may improve risk classification. Many other biomarkers are also associated with increased CVD risk, although few of these have been shown to be associated with appreciable reclassification. Total CV risk will also be higher than indicated in the SCORE charts in asymptomatic persons with abnormal markers of subclinical atherosclerotic vascular damage. Reclassification is of value in people identified as being at moderate $\mathrm{CV}$ risk by using markers such as CAC score $>100$ Agatston units, ankle-brachial index (ABI) $<0.9$ or $>1.40$, carotid-femoral pulse wave velocity $>10 \mathrm{~m} / \mathrm{s}$, or the presence of plaques at carotid or femoral ultrasonography. In studies comparing these markers, CAC had the best reclassification ability [14-16].

Some factors such as a high HDL-C up to $2.3 \mathrm{mmoL} / \mathrm{L}(90 \mathrm{mg} / \mathrm{dL})$ [17] or a family history of longevity can also be associated with lower risk.

\subsection{Risk levels}

A total CV risk estimate is part of a continuum. The cut-off points that are used to define high-risk are, in part, both arbitrary and based on the risk levels at which benefit is evident in clinical trials. In clinical practice, consideration should be given to practical issues in relation to the local healthcare systems. Not only should those at high risk be identified and managed, but those at moderate risk should also receive professional advice regarding lifestyle changes; in some cases, drug therapy will be needed to reduce atherosclerotic risk.

Low-risk people should be given advice to help them maintain this status. Thus, the intensity of preventive actions should be tailored to the patient's total CV risk. The strongest driver of total CV risk is age, which can be considered as 'exposure time' to risk factors.

For these reasons, the Task Force suggests the following categories of risk and LDL-C goals, based on the best available evidence and in an ideal setting with unlimited resources. These categories represent a counsel of perfection, but these ideals are for guidance only and practical decision-making must be based on what is appropriate to the local situation.

With these considerations, we propose the levels of total CV risk presented in Table 4.

\subsubsection{Role of non-invasive cardiovascular imaging techniques in the} assessment of total cardiovascular disease risk

Non-invasive imaging techniques can detect the presence, estimate the extent, and evaluate the clinical consequences of atherosclerotic vascular damage. Detection of coronary artery calcification with noncontrast computed tomography (CT) gives a good estimate of the atherosclerotic burden and is strongly associated with CV events [18]. A recent meta-analysis from the US Preventive Services Task Force summarized the available evidence on the value of non-traditional risk factors for risk prediction, and found that, although there are no randomized trials showing that the use of CAC reduces health outcomes, nevertheless it improves both discrimination and reclassification [19]. Assessment of carotid or femoral plaque burden with ultrasound has also been demonstrated to be predictive of $\mathrm{CV}$ events, comparable to CAC [20-23], while the measurement of the carotid intima-media thickness is inferior to CAC score and carotid plaque detection [16,24,25].

In asymptomatic patients at low or moderate risk who would be eligible for statin therapy (see Table 5), assessment of ASCVD with imaging may have an impact on medical treatment, both from the physician's and the patient's points of view. Data from the Multi-Ethnic Study of Atherosclerosis (MESA) showed that $41-57 \%$ of individuals who would be eligible for statin therapy had a CAC score of zero and the rate of atherosclerotic CVD events in the 10 year follow-up period was low (1.5-4.9\%) [26]. In contrast, the rates of ASCVD and coronary heart disease (CHD) events in individuals with a CAC score $>100$ Agatston were 18.9 and 12.7 per 1000 person-years, respectively [18]. Compared with a strategy of treating all patients, the use of CAC score to guide long-term statin therapy has been shown to be cost-effective [27]. Note that CAC score is often very low in patients younger than 45 years of age with severe familial hypercholesterolaemia (FH), including homozygous $\mathrm{FH}(\mathrm{HoFH})$, and has low specificity in this population.

Assessment of coronary luminal stenosis $>50 \%$ and plaque composition with coronary CT angiography also provides incremental prognostic value over traditional risk stratification models [28]. As a result, in asymptomatic individuals with moderate risk, the presence of a CAC score $>100$ Agatston, and carotid or femoral plaque burden on ultrasonography, may reclassify them to a higher risk category. Therefore, the use of methods to detect these markers should be of interest in that group (see Recommendations for cardiovascular imaging for risk assessment of atherosclerotic cardiovascular disease below) [14-16]. Overall, CAC score assessment with CT should be considered in individuals at low or moderate risk in whom the respective LDL-C goal is not achieved with lifestyle intervention alone, and pharmacological therapy is an option (see Table 5). The use of imaging techniques to determine the presence and extent of atherosclerotic vascular damage in low-risk individuals not being considered for statin therapy is not justified due to low prognostic yield, and the associated costs and radiation hazards when measuring CAC score, particularly among low-risk women [29]. Of note, CAC score is increased following statin treatment; therefore, the CAC scores of statin-treated patients should be interpreted with caution.

Recommenda tions for cardiovascular imaging for risk assessment of atherosclerotic cardiovascular disease.

\begin{tabular}{|c|c|c|}
\hline Recommendations & Class $^{\mathrm{a}}$ & Level $^{\mathrm{b}}$ \\
\hline $\begin{array}{l}\text { Arterial (carotid and/or femoral) plaque bur- } \\
\text { den on arterial ultrasonography should be } \\
\text { considered as a risk modifier in individuals at } \\
\text { low or moderate risk. }{ }^{29,30}\end{array}$ & Ila & B \\
\hline $\begin{array}{l}\text { CAC score assessment with } \mathrm{CT} \text { should be } \\
\text { considered as a risk modifier in the } \mathrm{CV} \text { risk } \\
\text { assessment of asymptomatic individuals at low } \\
\text { or moderate risk. }{ }^{14-16,24,26}\end{array}$ & Ila & B \\
\hline
\end{tabular}

$\mathrm{CAC}=$ coronary $\quad$ artery $\quad$ calcium $; \quad \mathrm{CT}=$ computed $\quad$ tomography; $\mathrm{CV}=$ cardiovascular.

${ }^{\text {a }}$ Class of recommendation.

b Level of evidence.

\subsubsection{Risk-based intervention strategies}

Table 5 presents suggested intervention strategies as a function of total CV risk and LDL-C level. This graded approach is based on evidence from multiple meta-analyses and individual randomized 
controlled trials (RCTs), which show a consistent and graded reduction in ASCVD risk in response to reductions in TC and LDL-C levels (see Recommendations for cardiovascular disease risk estimation below) [31-41]. These data are consistent in showing that, since the relative risk reduction is proportional to the absolute reduction in LDL-C and the absolute reduction in LDL-C resulting from a particular drug regimen depends only on baseline LDL-C, at any given level of baseline risk the higher the initial LDL-C level the greater the absolute reduction in risk. Advice on individual drug treatments is given in section 8 .

Recommendations for cardiovascular disease risk estimation.

\begin{tabular}{l|l|l|}
\hline Recommendations & Class & Level \\
\hline Total risk estimation using a risk estimation & \\
system such as SCORE is recommended for \\
asymptomatic adults $>40$ years of age without \\
evidence of CVD, DM, CKD, familial hyper- \\
cholesterolaemia, or LDL-C $>4.9$ mmol/L \\
(>190 mg/dL).
\end{tabular}

$\mathrm{CKD}=$ chronic kidney disease; $\mathrm{CV}=$ cardiovascular; $\mathrm{CVD}=$ cardiovascular disease; $\mathrm{DM}=$ diabetes mellitus; $\mathrm{FH}=$ familial hypercholesterolaemia; LDL$\mathrm{C}=$ low-density lipoprotein cholesterol; SCORE = Systematic Coronary Risk Estimation.

a Class of recommendation.

${ }^{\mathrm{b}}$ Level of evidence.

\section{Lipids and lipoproteins}

\subsection{Biological role of lipids and lipoproteins}

Lipoproteins in plasma transport lipids to tissues for energy utilization, lipid deposition, steroid hormone production, and bile acid formation. Lipoproteins consist of esterified and unesterified cholesterol, TGs, and phospholipids and protein components named apolipoproteins that act as structural components, ligands for cellular receptor binding, and enzyme activators or inhibitors.

There are six major lipoproteins in blood: chylomicrons, very lowdensity lipoprotein (VLDL), intermediate-density lipoprotein (IDL), LDL; Lp(a), and HDL (Table 6 and Supplementary Fig. 5).

\subsection{Role of lipids and lipoproteins in the pathophysiology of atherosclerosis}

All ApoB-containing lipoproteins $<70 \mathrm{~nm}$ in diameter, including smaller TG-rich lipoproteins and their remnant particles, can cross the endothelial barrier, especially in the presence of endothelial dysfunction, where they can become trapped after interaction with extracellular structures such as proteoglycans [42]. ApoB-containing lipoproteins retained in the arterial wall provoke a complex process that leads to lipid deposition and the initiation of an atheroma [43].

Continued exposure to ApoB-containing lipoproteins leads to additional particles being retained over time in the artery wall, and to the growth and progression of atherosclerotic plaques. On average, people with higher concentrations of plasma ApoB-containing lipoproteins will retain more particles and accumulate lipids faster, resulting in more rapid growth and the progression of atherosclerotic plaques.

Because atherosclerotic plaques grow over time as additional ApoBcontaining lipoprotein particles are retained, the size of the total atherosclerotic plaque burden is likely to be determined by both the concentration of circulating LDL-C and other ApoB-containing lipoproteins, and by the total duration of exposure to these lipoproteins. Therefore, a person's total atherosclerotic plaque burden is likely to be proportional to the cumulative exposure to these lipoproteins [44].

Eventually, the increase of the atherosclerotic plaque burden along with changes in the composition of the plaque reaches a critical point at which disruption of a plaque can result, with the formation of an overlying thrombus that acutely obstructs blood flow resulting in unstable angina, myocardial infarction (MI), or death. Therefore, the risk of experiencing an acute ASCVD event rises rapidly as more ApoBcontaining lipoproteins become retained and the atherosclerotic plaque burden increases. This provides the rationale for encouraging a healthy lifestyle to maintain low levels of ApoB-containing lipoproteins throughout life to slow the progression of atherosclerosis; it also explains the motivation to recommend treatment to lower LDL-C and other ApoB-containing lipoproteins, for both the primary prevention of ASCVD and the secondary prevention of recurrent CV events [44].

\subsection{Evidence for the causal effects of lipids and lipoproteins on the risk of atherosclerotic cardiovascular disease}

\subsubsection{Low-density lipoprotein cholesterol and risk of atherosclerosis}

Plasma LDL-C is a measure of the cholesterol mass carried by LDL particles, by far the most numerous of the ApoB-containing lipoproteins, and is an estimate of the concentration of circulating LDL. Numerous epidemiological studies, Mendelian randomization studies, and RCTs have consistently demonstrated a log-linear relationship between the absolute changes in plasma LDL-C and the risk of ASCVD [34,45-50]. The remarkable consistency among these studies, in addition to biological and experimental evidence, provides compelling evidence that LDL-C is causally associated with the risk of ASCVD, and that lowering LDL-C reduces the risk of ASCVD proportionally to the absolute achieved reduction in LDL-C [2,51].

Furthermore, Mendelian randomization studies have demonstrated that long-term exposure to lower LDL-C levels is associated with a much lower risk of $\mathrm{CV}$ events as compared with shorter-term exposure to lower LDL-C (as achieved, for example, in randomized trials) [48,52]. These data provide strong support for the concept that LDL particles have both a causal and cumulative effect on the risk of ASCVD. Therefore, the effect of LDL-C on the risk of ASCVD appears to be determined by both the absolute magnitude and the total duration of exposure to LDL-C [2].

The clinical benefit of lowering LDL-C is determined by the reduction in circulating LDL particles as estimated by ApoB, which is usually mirrored by a reduction of cholesterol carried by those particles [2,53]. Therefore, the clinical benefit of therapies that lower LDL-C by reducing LDL particle mass will be proportional to the absolute reduction in LDL$\mathrm{C}$, because- on average- the reduction in LDL-C and LDL particles will be concordant $[34,50,54,55]$. In contrast, the clinical benefit of therapies that lower LDL-C by a mechanism that may dramatically modify their composition may not be proportional to the observed absolute reduction in LDL-C, but instead would be expected to be proportional to the absolute change in LDL particle concentration as measured by a reduction in ApoB [2,53].

\subsubsection{Triglyceride-rich lipoproteins and risk of atherosclerosis}

TG-rich VLDL particles and their remnants carry most of the circulating TGs. Therefore, the plasma TG concentration reflects the concentration of circulating ApoB-containing TG-rich lipoproteins.

Elevated plasma TG levels are associated with an increasing risk of 
Table 5

Intervention strategies as a function of total cardiovascular risk and untreated low-density lipoprotein choles-terol levels.

\begin{tabular}{|c|c|c|c|c|c|c|c|}
\hline & \multirow{2}{*}{$\begin{array}{l}\text { Total CV risk } \\
\text { (SCORE) \% }\end{array}$} & \multicolumn{6}{|c|}{ Untreated LDL-C levels } \\
\hline & & $\begin{array}{l}<1.4 \mathrm{mmol} / \mathrm{L} \\
(55 \mathrm{mg} / \mathrm{dL})\end{array}$ & $\begin{array}{l}1.4 \text { to }<1.8 \\
\mathrm{mmol} / \mathrm{L}(55 \\
\text { to }<70 \mathrm{mg} / \mathrm{dL})\end{array}$ & $\begin{array}{l}1.8 \text { to }<2.6 \\
\mathrm{mmol} / \mathrm{L}(70 \\
\text { to }<100 \mathrm{mg} / \mathrm{dL})\end{array}$ & $\begin{array}{l}2.6 \text { to }<3.0 \\
\mathrm{mmol} / \mathrm{L}(100 \\
\text { to }<116 \mathrm{mg} / \mathrm{dL})\end{array}$ & $\begin{array}{l}3.0 \text { to }<4.9 \\
\mathrm{mmol} / \mathrm{L}(116 \text { to } \\
<190 \mathrm{mg} / \mathrm{dL})\end{array}$ & $\begin{array}{l}\geq 4.9 \mathrm{mmol} / \mathrm{L} \\
(\geq 190 \mathrm{mg} / \mathrm{dL})\end{array}$ \\
\hline \multirow[t]{8}{*}{$\begin{array}{l}\text { Primary } \\
\text { prevention }\end{array}$} & $<1$, low-risk & $\begin{array}{l}\text { Lifestyle } \\
\text { advice }\end{array}$ & Lifestyle advice & Lifestyle advice & Lifestyle advice & $\begin{array}{l}\text { Lifestyle inter- } \\
\text { vention, con- } \\
\text { sider adding } \\
\text { drug if } \\
\text { uncontrolled }\end{array}$ & $\begin{array}{l}\text { Lifestyle inter- } \\
\text { vention and } \\
\text { concomitant } \\
\text { drug } \\
\text { intervention }\end{array}$ \\
\hline & Class $^{\mathrm{a}} /$ Level $^{\mathrm{b}}$ & I/C & I/C & I/C & I/C & $\| \mathrm{la} / \mathrm{A}$ & $\mathrm{Ila} / \mathrm{A}$ \\
\hline & $\begin{array}{l}\geq 1 \text { to }<5, \text { or } \\
\text { moderate risk } \\
\text { (see Table } 4 \text { ) }\end{array}$ & $\begin{array}{l}\text { Lifestyle } \\
\text { advice }\end{array}$ & Lifestyle advice & Lifestyle advice & $\begin{array}{l}\text { Lifestyle inter- } \\
\text { vention, con- } \\
\text { sider adding } \\
\text { drug if } \\
\text { uncontrolled }\end{array}$ & $\begin{array}{l}\text { Lifestyle inter- } \\
\text { vention, con- } \\
\text { sider adding } \\
\text { drug if } \\
\text { uncontrolled }\end{array}$ & $\begin{array}{l}\text { Lifestyle inter- } \\
\text { vention and } \\
\text { concomitant } \\
\text { drug } \\
\text { intervention }\end{array}$ \\
\hline & Class $^{\mathrm{a}} /$ Level $^{\mathrm{b}}$ & $1 / C$ & I/C & $\mathrm{Ila} / \mathrm{A}$ & $\mathrm{Ila} / \mathrm{A}$ & $\mathrm{Ila} / \mathrm{A}$ & $\mathrm{Ila} / \mathrm{A}$ \\
\hline & $\begin{array}{l}\geq 5 \text { to }<10, \text { or } \\
\text { high-risk } \\
\text { (see Table } 4 \text { ) }\end{array}$ & $\begin{array}{l}\text { Lifestyle } \\
\text { advice }\end{array}$ & Lifestyle advice & $\begin{array}{l}\text { Lifestyle inter- } \\
\text { vention, con- } \\
\text { sider adding } \\
\text { drug if } \\
\text { uncontrolled }\end{array}$ & $\begin{array}{l}\text { Lifestyle inter- } \\
\text { vention and con- } \\
\text { comitant drug } \\
\text { intervention }\end{array}$ & $\begin{array}{l}\text { Lifestyle inter- } \\
\text { vention and } \\
\text { concomitant } \\
\text { drug } \\
\text { intervention }\end{array}$ & $\begin{array}{l}\text { Lifestyle inter- } \\
\text { vention and } \\
\text { concomitant } \\
\text { drug } \\
\text { intervention }\end{array}$ \\
\hline & Class $^{a} /$ Level $^{\mathrm{b}}$ & $\mathrm{Ila} / \mathrm{A}$ & $\mathrm{Ila} / \mathrm{A}$ & $\mathrm{Ila} / \mathrm{A}$ & I/A & I/A & I/A \\
\hline & $\begin{array}{l}\geq 10 \text {, or at } \\
\text { very-high } \\
\text { risk due } \\
\text { to a risk condi- } \\
\text { tion } \\
\text { (see Table 4) }\end{array}$ & $\begin{array}{l}\text { Lifestyle } \\
\text { advice }\end{array}$ & $\begin{array}{l}\text { Lifestyle inter- } \\
\text { vention, con- } \\
\text { sider adding } \\
\text { drug if } \\
\text { uncontrolled }\end{array}$ & $\begin{array}{l}\text { Lifestyle inter- } \\
\text { vention and } \\
\text { concomitant } \\
\text { drug } \\
\text { intervention }\end{array}$ & $\begin{array}{l}\text { Lifestyle inter- } \\
\text { vention and con- } \\
\text { comitant drug } \\
\text { intervention }\end{array}$ & $\begin{array}{l}\text { Lifestyle inter- } \\
\text { vention and } \\
\text { concomitant } \\
\text { drug } \\
\text { intervention }\end{array}$ & $\begin{array}{l}\text { Lifestyle inter- } \\
\text { vention and } \\
\text { concomitant } \\
\text { drug } \\
\text { intervention }\end{array}$ \\
\hline & Class $^{a} /$ Level $^{b}$ & $\mathrm{Ila} / \mathrm{B}$ & $\mathrm{Ila} / \mathrm{A}$ & I/A & I/A & I/A & I/A \\
\hline \multirow[t]{2}{*}{$\begin{array}{l}\text { Secondary } \\
\text { prevention }\end{array}$} & Very-high-risk & $\begin{array}{l}\text { Lifestyle inter- } \\
\text { vention, con- } \\
\text { sider adding } \\
\text { drug if } \\
\text { uncontrolled }\end{array}$ & $\begin{array}{l}\text { Lifestyle inter- } \\
\text { vention and } \\
\text { concomitant } \\
\text { drug } \\
\text { intervention }\end{array}$ & $\begin{array}{l}\text { Lifestyle inter- } \\
\text { vention and } \\
\text { concomitant } \\
\text { drug } \\
\text { intervention }\end{array}$ & $\begin{array}{l}\text { Lifestyle inter- } \\
\text { vention and con- } \\
\text { comitant drug } \\
\text { intervention }\end{array}$ & $\begin{array}{l}\text { Lifestyle inter- } \\
\text { vention and } \\
\text { concomitant } \\
\text { drug } \\
\text { intervention }\end{array}$ & $\begin{array}{l}\text { Lifestyle inter- } \\
\text { vention and } \\
\text { concomitant } \\
\text { drug } \\
\text { intervention } \\
\end{array}$ \\
\hline & Class $^{\mathrm{a}} /$ Level $^{\mathrm{b}}$ & $\mathrm{Ila} / \mathrm{A}$ & I/A & I/A & I/A & I/A & I/A \\
\hline
\end{tabular}

ASCVD, but this association becomes null after adjusting for non-HDL$\mathrm{C}$, an estimate of the total concentration of all ApoB-containing lipoproteins [45]. Similarly, lowering TG with fibrates reduces the risk of $\mathrm{CV}$ events by the same amount as LDL-C-lowering therapies when measured per unit change of non-HDL-C [50], suggesting that the effect of plasma TGs on ASCVD is mediated by changes in the concentration of TG-rich lipoproteins as estimated by non-HDL-C.

Mendelian randomization studies also suggest that the association between plasma TGs and the risk of CHD may be causal; however, this evidence must be interpreted with caution because nearly all variants associated with TGs are also associated with HDL-C, LDL-C, or Lp(a) [56-59]. A recent Mendelian randomization study demonstrated that TG-lowering lipoprotein lipase (LPL) variants and LDL-C-lowering LDL receptor variants had the same effect on the risk of ASCVD per unit change of ApoB, suggesting that all ApoB-containing lipoproteins have the same effect on the risk of CHD [53]. Together, these studies strongly suggest that the causal effect of TG-rich lipoproteins and their remnants on the risk of ASCVD is determined by the circulating concentration of ApoB-containing particles, rather than by the TG content itself.

\subsubsection{High-density lipoprotein cholesterol and risk of atherosclerosis}

The inverse association between plasma HDL-C and the risk of ASCVD is among the most consistent and reproducible associations in observational epidemiology $[45,60]$. In contrast, Mendelian randomization studies do not provide compelling evidence that HDL-C is causally associated with the risk of ASCVD [49,61,62]. However, this evidence must be interpreted with caution because most genetic variants associated with HDL-C are also associated with directionally opposite changes in TGs, LDL-C, or both, thus making estimates of the effect of HDL-C on the risk of ASCVD very difficult using the Mendelian randomization study design. Furthermore, there is no evidence from randomized trials that therapeutically increasing plasma HDL-C reduces the risk of CV events [63-67]. In the Effects of Dalcetrapib in Patients with a Recent Acute Coronary Syndrome (dal-OUTCOMES) trial, treatment with the cholesteryl ester transfer protein (CETP) inhibitor dalcetrapib increased HDL-C without any effect on LDL-C or ApoB, but did not reduce the risk of major CV events [65]. Similarly, in the Assessment of Clinical Effects of Cholesteryl Ester Transfer Protein Inhibition with Evacetrapib in Patients at a High-Risk for Vascular Outcomes (ACCELERATE) and Randomized Evaluation of the Effects of Anacetrapib Through Lipid Modification (REVEAL) trials, treatment with CETP inhibitors more than doubled HDL-C levels, but did not appear to reduce the risk of ASCVD events beyond that expected from the modest reductions in ApoB levels [2,63,64]. Furthermore, several randomized trials have shown that directly infused HDL mimetics increase plasma HDL-C concentrations, but do not reduce the progression of atherosclerosis as measured by intravascular ultrasound $[68,69]$.

Therefore, there is currently no randomized trial or genetic evidence to suggest that raising plasma HDL-C is likely to reduce the risk of ASCVD events. Whether therapies that alter the function of HDL particles will reduce the risk of ASCVD is unknown.

5.3.4. Lipoprotein (a) and risk of atherosclerosis

Lp(a) is an LDL particle with an Apo(a) moiety covalently bound to 
its ApoB component [70]. It is $<70 \mathrm{~nm}$ in diameter and can freely flux across the endothelial barrier, where it can become-similarly to LDL-retained within the arterial wall and thus may increase the risk of ASCVD. Pro-atherogenic effects of $\operatorname{Lp}(\mathrm{a})$ have also been attributed to pro-coagulant effects as $\mathrm{Lp}(\mathrm{a})$ has a similar structure to plasminogen, and it has pro-inflammatory effects most likely related to the oxidized phospholipid load carried by Lp(a) [71].

Higher plasma Lp(a) concentrations are associated with an increased risk of ASCVD, but it appears to be a much weaker risk factor for most people than LDL-C $[72,73]$. In contrast, Mendelian randomization studies have consistently demonstrated that lifelong exposure to higher Lp(a) levels is strongly and causally associated with an increased risk of ASCVD $[74,75]$. While randomized trials evaluating therapies that lower $\mathrm{Lp}(\mathrm{a})$ by $20-30 \%$ (including niacin and CETP inhibitors) have not provided evidence that lowering $\mathrm{Lp}$ (a) reduces the risk of ASCVD beyond that which would be expected from the observed reduction in ApoB-containing lipoproteins, recent data with PCSK9 inhibitors have suggested a possible role for $\mathrm{Lp}(\mathrm{a})$ lowering in reducing $\mathrm{CV}$ risk [76].

This conflicting evidence appears to have been reconciled by a recent Mendelian randomization study that showed that the causal effect of $\mathrm{Lp}(\mathrm{a})$ on the risk of ASCVD is proportional to the absolute change in plasma Lp(a) levels. Importantly, this study also suggested that people with extremely high Lp(a) levels $>180 \mathrm{mg} / \mathrm{dL}$ ( $>430 \mathrm{nmoL} / \mathrm{L}$ ) may have an increased lifetime risk of ASCVD similar to that of people with heterozygous $\mathrm{FH}(\mathrm{HeFH})$. Because about $90 \%$ of a person's Lp(a) level is inherited, extremely elevated $\mathrm{Lp}$ (a) may represent a new inherited lipid disorder that is associated with extremely high lifetime risk of ASCVD and is two-fold more prevalent than HeFH [77]. However, this study [77] and another based on the Heart Protection Study 2-Treatment of HDL to Reduce the Incidence of Vascular Events (HPS2-THRIVE) trial [78] have shown that large absolute changes in Lp(a) may be needed to produce a clinically meaningful reduction in the risk of ASCVD events.

\subsection{Laboratory measurement of lipids and lipoproteins}

Measurement of lipids and lipoproteins is used to estimate the risk of ASCVD and guide therapeutic decision-making. Quantification of plasma lipids can be performed on whole plasma and quantification of lipoproteins can be achieved by measuring their protein component. Operationally, lipoproteins are classified based on their hydrated density (see Table 6).

\subsubsection{Lipoprotein measurement}

Given the central causal role of ApoB-containing lipoproteins in the initiation and progression of atherosclerosis, direct measurement of the circulating concentration of atherogenic ApoB-containing lipoproteins to both estimate risk and guide treatment decisions would be ideal. Because all ApoB-containing lipoproteins-including VLDL, TG-rich remnant particles, and LDL-contain a single ApoB molecule, quantitation of ApoB directly estimates the number of atherogenic particles in plasma.

Standardized, automated, accurate, and inexpensive methods to measure ApoB are available. Fasting is not required because even in the post-prandial state, ApoB48-containing chylomicrons typically represent $<1 \%$ of the total concentration of circulating ApoB-containing lipoproteins. Furthermore, the analytical performances of ApoB measurement methods are superior to the measurement or calculation of LDL-C and non-HDL-C [79].

\subsubsection{Lipid measurements}

In clinical practice, the concentration of plasma lipoproteins is not usually measured directly but is instead estimated by measuring their cholesterol content. TC in humans is distributed primarily among three major lipoprotein classes: VLDL, LDL, and HDL. Smaller amounts of cholesterol are also contained in two minor lipoprotein classes: IDL and $\mathrm{Lp}(\mathrm{a})$. A standard serum lipid profile measures the concentration of TC and HDL-C, as well as TG. With these values, the LDL-C concentration can be estimated.

Plasma LDL-C can be measured directly using enzymatic techniques or preparative ultracentrifugation, but in clinical medicine it is most often calculated using the Friedewald formula:

LDL-C $=$ TC - HDL-C $-(T G / 2.2)$ in $\mathrm{mmol} / \mathrm{L}$

or

LDL-C $=$ TC - HDL-C $-(\mathrm{TG} / 5)$ in $\mathrm{mg} / \mathrm{dL}$

Although convenient, the Friedewald calculated value of LDL-C has several well-established limitations: (i) methodological errors may accumulate since the formula necessitates three separate analyses of TC, TGs, and HDL-C; and (ii) a constant cholesterol/TG ratio in VLDL is assumed. With high TG values ( $>4.5 \mathrm{mmoL} / \mathrm{L}$ or $>400 \mathrm{mg} / \mathrm{dL}$ ) the formula cannot be used. This should especially be considered in non-fasting samples.

To overcome the problems associated with calculated LDL-C, direct enzymatic methods for the measurement of LDL-C have been developed. These methods are commercially available as ready to use tools for automatic analysis. The definition of LDL-C by the Friedewald equation and by direct measurement is the same: non-HDL-C - VLDL-C, representing the sum of the cholesterol carried by the biochemically defined LDL, IDL, and Lp(a) subfractions.

For the general population, calculated LDL-C and direct LDL-C show very strong correlations [80-83]. However, calculated LDL-C has been found to underestimate LDL-C at concentrations of TG $\geq 2 \mathrm{mmoL} / \mathrm{L}$ $(177 \mathrm{mg} / \mathrm{dL})[81,82]$. Equally, at very low levels of LDL-C, calculated LDL-C may be misleading, especially in the presence of high TG [81,84-86]. To avoid some of the problems with the Friedewald formula, a number of modifications for the calculation of LDL-C have been suggested, but it remains to be proved whether these modifications are superior to Friedewald's formula for the estimation of $\mathrm{CV}$ risk [81,85-87]. It is important to note that direct LDL-C measurements also have limitations, including systematic bias and inaccuracy in patients with dyslipidaemia, especially for high TG levels [88-90].

As an alternative calculated LDL-C, non-HDL-C can be calculated as TC - HDL-C and is a measure of the TC carried by all atherogenic ApoBcontaining lipoproteins, including TG-rich particles in VLDL and their remnants [100].

Several methods for the determination of $\operatorname{Lp}(a)$ are available. The complex molecular structure of $\mathrm{Lp}(\mathrm{a})$ and the variation in size of Apo(a) has been a challenge in the development of analytical methods for Lp (a). Available methods are, to a varying degree, influenced by the Apo (a) isoform [91]. Furthermore, the concentration of $\mathrm{Lp}(\mathrm{a})$ is reported as either a molar concentration $(\mathrm{nmol} / \mathrm{L})$ or as a mass $(\mathrm{mg} / \mathrm{dL})$ by the various assays, and conversion between molar and mass concentrations has been found to be both size- and concentration-dependent [91-93]. Therefore, standardization between assays is needed to establish a reliable and reproducible method for the quantification of $\mathrm{Lp}(\mathrm{a})$ mass or particle number [92].

\subsubsection{Fasting or non-fasting?}

Traditionally, blood sampling for lipid analyses has been recommended in the fasting state. Recent systematic studies comparing fasting and non-fasting samples have suggested that the difference is small for most lipid parameters [85,94-100]. Non-fasting sampling has been used in large population-based studies [100]. In most studies, nonfasting samples display a higher TG level of $\sim 0.3 \mathrm{mmoL} / \mathrm{L}(27 \mathrm{mg} / \mathrm{dL})$ $[100,101]$. On average, and for most individuals, this increment will be of no clinical significance. Indeed, a number of guidelines recommend non-fasting sampling [100,102,103].

For general risk screening, non-fasting samples seem to have at least the same prognostic value as fasting samples [104]. The practical advantages of non-fasting samples, including better patient acceptability, outweigh the potential imprecision in some patients, although the determination of some key analytes, such as fasting glucose, may be compromised. Furthermore, 
even if non-fasting sampling can be used in most cases, in patients with metabolic syndrome (MetS), DM, or hypertriglyceridaemia (HTG), calculated LDL-C should be interpreted with caution.

\subsection{Recommendations for measuring lipids and lipoproteins to estimate risk of atherosclerotic cardiovascular disease}

Measurement of plasma TC is needed to calculate risk using SCORE, while the inclusion of plasma HDL-C level can improve risk estimation using the online SCORE calculator. Therefore, both TC and HDL-C should be measured to estimate a person's risk of ASCVD using SCORE, or one of the other risk calculators (almost all of which also include measurements of TC and HDL-C).

Plasma LDL-C should be measured to estimate the risk of ASCVD that can be modified with LDL-C-lowering therapies, and to identify whether markedly elevated LDL-C levels are present that may suggest a lifetime high-risk of ASCVD due to lifelong cumulative exposure to high levels of atherogenic lipoproteins, such as in FH. Plasma LDL-C can be either calculated or measured directly.

Plasma TG should be assessed to identify people who may have a greater modifiable risk of ASCVD than is reflected by LDL-C, due to the presence of an increased concentration of atherogenic ApoB-containing TG-rich lipoproteins and their remnants, and to identify people in whom calculated and directly measured LDL-C may underestimate the risk of ASCVD by underestimating either the concentration of circu- be discordance between measured LDL-C and ApoB levels [85,109].

Considering the potential inaccuracy of LDL-C in dyslipidaemia, among patients with DM or high TG levels, and in patients with very low LDL-C levels, measurement of both ApoB and non-HDL-C is recommended as part of routine lipid analysis for risk evaluation in patients with elevated plasma TGs. Because ApoB provides an accurate estimate of the total concentration of atherogenic particles under all circumstances, it is the preferred measurement to further refine the estimate of ASCVD risk that is modifiable by lipid-lowering therapy.

$\mathrm{Lp}$ (a) has a similar structure to plasminogen and binds to the plasminogen receptor, leading to increased thrombosis (pro-thrombotic factor). Measurement of $\mathrm{Lp}(\mathrm{a})$ should be considered at least once in each person's lifetime, if available, to identify people who have inherited an extremely elevated level of $\mathrm{Lp}(\mathrm{a}) \geq 180 \mathrm{mg} / \mathrm{dL}(\geq 430$ $\mathrm{nmoL} / \mathrm{L}$ ) and therefore have a very high lifetime risk of ASCVD that is approximately equivalent to the risk associated with $\mathrm{HeFH}$. In addition, this strategy can identify people with less-extreme Lp(a) elevations who may be at a higher risk of ASCVD, which is not reflected by the SCORE system, or by other lipid or lipoprotein measurements. Measurement of Lp(a) has been shown to provide clinically significant improved risk reclassification under certain conditions, and therefore should be considered in patients who have an estimated 10-year risk of ASCVD that is close to the threshold between high and moderate risk [110-112].

Recommendations for measuring lipids and lipoproteins to estimate the risk of ASCVD are summarized below.

Recommendations for lipid analyses for cardiovascular disease risk estimation.

\begin{tabular}{|c|c|c|}
\hline Recommendations & Class $^{a}$ & Level $^{\mathrm{b}}$ \\
\hline $\mathrm{TC}$ is to be used for the estimation of total CV risk by means of the SCORE system. & I & C \\
\hline HDL-C analysis is recommended to further refine risk estimation using the online SCORE system. & I & C \\
\hline LDL-C analysis is recommended as the primary lipid analysis method for screening, diagnosis, and management. & I & C \\
\hline TG analysis is recommended as part of the routine lipid analysis process. & I & C \\
\hline $\begin{array}{l}\text { Non-HDL-C evaluation is recommended for risk assessment, particularly in people with high TG levels, DM, obesity, or } \\
\text { very low LDL-C levels. }\end{array}$ & I & C \\
\hline $\begin{array}{l}\text { ApoB analysis is recommended for risk assessment, particularly in people with high TG levels, DM, obesity, metabolic syn- } \\
\text { drome, or very low LDL-C levels. It can be used as an alternative to LDL-C, if available, as the primary measurement for } \\
\text { screening, diagnosis, and management, and may be preferred over non-HDL-C in people with high TG levels, DM, obesity, } \\
\text { or very low LDL-C levels. }\end{array}$ & I & C \\
\hline $\begin{array}{l}\mathrm{Lp}(\mathrm{a}) \text { measurement should be considered at least once in each adult person's lifetime to identify those with very high } \\
\text { inherited } \mathrm{Lp}(\mathrm{a}) \text { levels }>180 \mathrm{mg} / \mathrm{dL}(>430 \mathrm{nmol} / \mathrm{L}) \text { who may have a lifetime risk of ASCVD equivalent to the risk associated } \\
\text { with heterozygous familial hypercholesterolaemia. }\end{array}$ & Ila & C \\
\hline $\begin{array}{l}\text { Lp(a) should be considered in selected patients with a family history of premature CVD, and for reclassification in people } \\
\text { who are borderline between moderate and high-risk. }\end{array}$ & Ila & C \\
\hline
\end{tabular}

Apo = apolipoprotein; ASCVD = atherosclerotic cardiovascular disease; CV = cardiovascular; CVD = cardiovascular disease; DM = diabetes mellitus; HDL$\mathrm{C}=$ high-density lipoprotein cholesterol; LDL-C = low-density lipoprotein cholesterol; Lp(a) = lipoprotein(a); SCORE = Systematic Coronary Risk Estimation; $\mathrm{TC}=$ total cholesterol; TG $=$ triglyceride.

lating LDL particles or the cholesterol content carried by those particles, such as those with very low levels of LDL. This may be especially relevant in patients with DM or MetS.

In general, LDL-C, non-HDL-C, and ApoB concentrations are very highly correlated. As a result, under most circumstances, they provide very similar information about ASCVD risk [45,105-108]. However, under certain circumstances-including among people with elevated TG levels, DM, obesity, or very low achieved LDL-C levels-the calculated or directly measured LDL-C level may underestimate both the total concentration of cholesterol carried by LDL and, more importantly, underestimate the total concentration of ApoB-containing lipoproteins, thus underestimating the risk of ASCVD. In around $20 \%$ of patients there may

\section{Treatment targets and goals}

In previous EAS/ESC Guidelines for the management of dyslipidaemias $[1,113]$ and other major guidelines on the treatment of blood cholesterol to reduce atherosclerotic CV risk in adults $[40,114]$, the importance of LDL-C lowering to prevent ASCVD is strongly emphasized. The European Task Force felt that limiting the current knowledge on CV prevention only to results from RCTs reduces the exploitation of the potential that is available for the prevention of ASCVD. It is the concordance of the conclusions from many different approaches (from basic science, clinical observations, genetics, epidemiology, RCTs, etc.) that contributes to the understanding of the causes of ASCVD and to the 
Table 6

Physical and chemical characteristics of human plasma lipoproteins.

\begin{tabular}{|c|c|c|c|c|c|c|c|c|}
\hline & \multirow[t]{2}{*}{ Density $(\mathrm{g} / \mathrm{mL})$} & \multirow{2}{*}{$\frac{\text { Diameter }}{(\mathrm{nm})}$} & \multirow[t]{2}{*}{ TGs (\%) } & \multirow{2}{*}{$\begin{array}{l}\text { Cholesteryl } \\
\text { esters (\%) }\end{array}$} & \multirow[t]{2}{*}{ PLs (\%) } & \multirow[t]{2}{*}{ Cholesterol (\%) } & \multicolumn{2}{|c|}{ Apolipoproteins } \\
\hline & & & & & & & Major & Others \\
\hline Chylomicrons & $<0.95$ & $80-100$ & $90-95$ & $2-4$ & $2-6$ & 1 & ApoB-48 & ApoA-I, A-II, A-IV, A-V \\
\hline VLDL & $0.95-1.006$ & $30-80$ & $50-65$ & $8-14$ & $12-16$ & $4-7$ & ApoB-100 & ApoA-I, C-II, C-III, E, A-V \\
\hline IDL & $1.006-1.019$ & $25-30$ & $25-40$ & $20-35$ & $16-24$ & $7-11$ & ApoB-100 & ApoC-II, C-III, E \\
\hline LDL & $1.019-1.063$ & $20-25$ & $4-6$ & $34-35$ & $22-26$ & $6-15$ & ApoB-100 & \\
\hline HDL & $1.063-1.210$ & $8-13$ & 7 & $10-20$ & 55 & 5 & ApoA-I & ApoA-II, C-III, E, M \\
\hline $\mathrm{Lp}(\mathrm{a})$ & $1.006-1.125$ & $25-30$ & $4-8$ & $35-46$ & $17-24$ & $6-9$ & Apo(a) & ApoB-100 \\
\hline
\end{tabular}

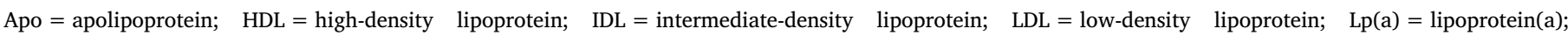
PLs = phospholipids; TGs = triglycerides; VLDL = very low-density lipoprotein.

Table 7

Treatment targets and goals for cardiovascular disease prevention.

\begin{tabular}{|c|c|}
\hline Smoking & No exposure to tobacco in any form. \\
\hline Diet & Healthy diet low in saturated fat with a focus on wholegrain products, vegetables, fruit, and fish. \\
\hline Physical activity & 3.5-7 h moderately vigorous physical activity per week or 30-60 min most days. \\
\hline Body weight & BMI $20-25 \mathrm{~kg} / \mathrm{m}^{2}$, and waist circumference $<94 \mathrm{~cm}$ (men) and $<80 \mathrm{~cm}$ (women). \\
\hline Blood pressure & $<140 / 90 \mathrm{mmHg}^{\mathrm{a}}$ \\
\hline \multirow[t]{8}{*}{ LDL-C } & Very-high risk in primary or secondary prevention: \\
\hline & A therapeutic regimen that achieves $\geq 50 \%$ LDL-C reduction from baseline $\mathrm{b}^{\mathrm{b}}$ and an LDL-C goal of $<1.4 \mathrm{mmoL} / \mathrm{L}(<55 \mathrm{mg} / \mathrm{dL})$. \\
\hline & No current statin use: this is likely to require high-intensity LDL-lowering therapy. \\
\hline & Current LDL-lowering treatment: an increased treatment intensity is required. \\
\hline & $\begin{array}{l}\text { High risk: A therapeutic regimen that achieves } \geq 50 \% \text { LDL-C reduction from baseline }{ }^{\mathrm{b}} \text { and an } \mathrm{LDL}-\mathrm{C} \text { goal of }<1.8 \mathrm{mmoL} / \mathrm{L}(<70 \mathrm{mg} / \mathrm{dL}) \text {. } \\
\text { Moderate risk: }\end{array}$ \\
\hline & A goal of $<2.6 \mathrm{mmoL} / \mathrm{L}(<100 \mathrm{mg} / \mathrm{dL})$ \\
\hline & Low risk: \\
\hline & A goal of $<3.0 \mathrm{mmoL} / \mathrm{L}(<116 \mathrm{mg} / \mathrm{dL})$ \\
\hline Non-HDL-C & Non-HDL-C secondary goals are $<2.2,2.6$, and $3.4 \mathrm{mmoL} / \mathrm{L}(<85,100$, and $130 \mathrm{mg} / \mathrm{dL})$ for very-high-, high-, and moderate-risk people, respectively. \\
\hline ApoB & ApoB secondary goals are $<65,80$, and $100 \mathrm{mg} / \mathrm{dL}$ for very-high-, high-, and moderate-risk people, respectively. \\
\hline Triglycerides & No goal, but $<1.7 \mathrm{mmoL} / \mathrm{L}(<150 \mathrm{mg} / \mathrm{dL})$ indicates lower risk and higher levels indicate a need to look for other risk factors. \\
\hline Diabetes & HbA1c: $<7 \%(<53 \mathrm{mmoL} / \mathrm{mol})$ \\
\hline
\end{tabular}

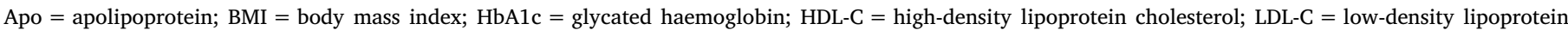
cholesterol.

a Lower treatment targets are recommended for most treated hypertensive patients, provided that the treatment is well tolerated [118].

b The term 'baseline' refers to the LDL-C level in a person not taking any lipid-lowering medication, or to the extrapolated baseline value for those who are on current treatment.

potential of prevention. The Task Force is aware of the limitations of some of the sources of evidence and accepts that RCTs have not examined different LDL-C goals systematically, but felt that it was appropriate to look at the totality of the evidence. Particular consideration was given to results from meta-analyses confirming the dose-dependent reduction in ASCVD with LDL-C-lowering agents; the greater the absolute LDL-C reduction, the greater the $\mathrm{CV}$ risk reduction $[35,36,50,115]$. The benefits related to LDL-C reduction are not specific for statin therapy [33]. No level of LDL-C below which benefit ceases or harm occurs has been defined.

There is considerable individual variability in the LDL-C response to dietary and drug treatments [31], which is traditionally taken to support a tailored approach to management. Total CV risk reduction should be individualized, and this can be more specific if goals are defined. The use of goals can also aid patient-doctor communication. It is judged that a goal approach may facilitate adherence to treatment, although this consensus opinion has not been fully tested. For all these reasons, the European Task Force retains a goal approach to lipid management and treatment goals are tailored to the total CV risk level. There is also evidence suggesting that lowering of LDL-C beyond the goals that were set in the previous EAS/ESC Guidelines is associated with fewer ASCVD events $[34,116,117]$. Therefore, it seems appropriate to reduce LDL-C to as low a level as possible, at least in patients at very high CV risk, and for this reason a minimum $50 \%$ reduction is suggested for LDL reduction, together with reaching the tailored goal.

The lipid goals are part of a comprehensive CV risk reduction strategy and are summarized in Table 7 . The rationales for the non-lipid targets are given in the 2016 ESC Joint Prevention Guidelines [10].

The targeted approach to lipid management is primarily aimed at reducing atherosclerotic risk by substantially lowering LDL-C to levels that have been achieved in recent large-scale trials of PCSK-9 inhibitors. Therefore, for patients at very high CV risk, whether in secondary prevention or (rarely) in primary prevention, LDL-C reduction of $\geq 50 \%$ from baseline and an LDL-C goal of $<1.4 \mathrm{mmoL} / \mathrm{L}(<55 \mathrm{mg} / \mathrm{dL})$ are recommended. For patients with ASCVD who experience a second vascular event within 2 years (not necessarily of the same type as the first event) while taking maximally tolerated statin-based therapy, an LDL-C goal $<1.0 \mathrm{mmoL} / \mathrm{L} \quad(<40 \mathrm{mg} / \mathrm{dL})$ may be considered $[119,120]$. For people at high CV risk, an LDL-C reduction of $\geq 50 \%$ from baseline and an LDL-C goal $<1.8 \mathrm{mmoL} / \mathrm{L}(<70 \mathrm{mg} / \mathrm{dL})$ are recommended. In patients at moderate $\mathrm{CV}$ risk, an LDL-C goal $<2.6$ $\mathrm{mmoL} / \mathrm{L}(<100 \mathrm{mg} / \mathrm{dL})$ should be considered, while for low-risk individuals a goal of $<3.0 \mathrm{mmoL} / \mathrm{L}(<116 \mathrm{mg} / \mathrm{dL})$ may be considered (see Recommendations for treatment goals for low-density lipoprotein cholesterol below and Supplementary Table 2). 
Recommendations for treatment goals for low-density lipoprotein cholesterol.

\begin{tabular}{|c|c|c|}
\hline Recommendations & Class $^{\mathrm{a}}$ & Level $^{\mathrm{b}}$ \\
\hline $\begin{array}{l}\text { In secondary prevention for patients at very-high risk, }{ }^{c} \text { an LDL-C reduction of } \geq 50 \% \text { from baseline }{ }^{\mathrm{d}} \text { and an LDL-C goal of } \\
<1.4 \mathrm{mmol} / \mathrm{L}(<55 \mathrm{mg} / \mathrm{dL}) \text { are recommended. }{ }^{33-35,119,120}\end{array}$ & $\mathbf{I}$ & A \\
\hline $\begin{array}{l}\text { In primary prevention for individuals at very-high risk but without } \mathrm{FH},{ }^{\mathrm{c}} \text { an } \mathrm{LDL}-\mathrm{C} \text { reduction of } \geq 50 \% \text { from baseline }{ }^{\mathrm{d}} \text { and } \\
\text { an } \mathrm{LDL}-\mathrm{C} \text { goal of }<1.4 \mathrm{mmol} / \mathrm{L}(<55 \mathrm{mg} / \mathrm{dL}) \text { are recommended. }{ }^{34-36}\end{array}$ & I & C \\
\hline $\begin{array}{l}\text { In primary prevention for individuals with } \mathrm{FH} \text { at very-high risk, an } \mathrm{LDL}-\mathrm{C} \text { reduction of } \geq 50 \% \text { from baseline and an } \mathrm{LDL}-\mathrm{C} \\
\text { goal of }<1.4 \mathrm{mmol} / \mathrm{L}(<55 \mathrm{mg} / \mathrm{dL} \text { ) should be considered. }\end{array}$ & Ila & C \\
\hline $\begin{array}{l}\text { For patients with ASCVD who experience a second vascular event within } 2 \text { years (not necessarily of the same type as the } \\
\text { first event) while taking maximally tolerated statin-based therapy, an LDL-C goal of }<1.0 \mathrm{mmol} / \mathrm{L}(<40 \mathrm{mg} / \mathrm{dL} \text { ) may be } \\
\text { considered. }{ }^{119,120}\end{array}$ & Illb & B \\
\hline $\begin{array}{l}\text { In patients at high risk, }{ }^{\mathrm{c}} \text { an } L D L-C \text { reduction of } \geq 50 \% \text { from baseline }{ }^{\mathrm{d}} \text { and an } \mathrm{LDL}-\mathrm{C} \text { goal of }<1.8 \mathrm{mmol} / \mathrm{L}(<70 \mathrm{mg} / \mathrm{dL}) \text { are } \\
\text { recommended. }{ }^{34,35}\end{array}$ & I & A \\
\hline In individuals at moderate risk, ${ }^{c}$ an LDL-C goal of $<2.6 \mathrm{mmol} / \mathrm{L}(<100 \mathrm{mg} / \mathrm{dL})$ should be considered. ${ }^{34}$ & Ila & A \\
\hline In individuals at low risk, ${ }^{c}$ an $\mathrm{LDL}-\mathrm{C}$ goal $<3.0 \mathrm{mmol} / \mathrm{L}(<116 \mathrm{mg} / \mathrm{dL})$ may be considered. ${ }^{36}$ & IIb & A \\
\hline
\end{tabular}

ASCVD = atherosclerotic cardiovascular disease; $\mathrm{FH}=$ familial hypercholesterolaemia; LDL-C = low-density lipoprotein cholesterol.

a Class of recommendation.

${ }^{\mathrm{b}}$ Level of evidence.

${ }^{\mathrm{c}}$ For definitions see Table 4.

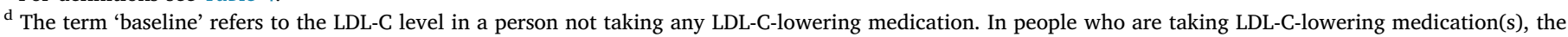

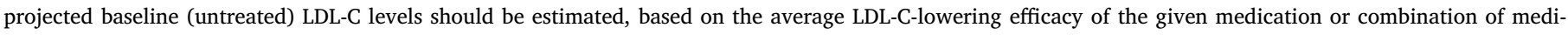
cations.

Table 8

Impact of specific lifestyle changes on lipid levels.

\begin{tabular}{|c|c|c|c|}
\hline & Magnitude of the effect & Level & Reference \\
\hline \multicolumn{4}{|l|}{ Lifestyle interventions to reduce TC and LDL-C levels } \\
\hline Avoid dietary trans fats & ++ & A & 129,138 \\
\hline Reduce dietary saturated fats & ++ & A & 129,139 \\
\hline Increase dietary fibre & ++ & A & 140,141 \\
\hline Use functional foods enriched with phytosterols & ++ & A & 142,143 \\
\hline Use red yeast rice nutraceuticals & ++ & A & $144-146$ \\
\hline Reduce excessive body weight & ++ & A & 147,148 \\
\hline Reduce dietary cholesterol & + & B & 149,150 \\
\hline Increase habitual physical activity & + & B & 151 \\
\hline \multicolumn{4}{|l|}{ Lifestyle interventions to reduce TG-rich lipoprotein levels } \\
\hline Reduce excessive body weight & + & A & 147,148 \\
\hline Reduce alcohol intake & +++ & A & 152,153 \\
\hline Increase habitual physical activity & ++ & A & 151,154 \\
\hline Reduce total amount of dietary carbohydrates & ++ & A & 147,155 \\
\hline Use supplements of $n-3$ polyunsaturated fats & ++ & A & 156,157 \\
\hline Reduce intake of mono- and disaccharides & ++ & B & 158,159 \\
\hline Replace saturated fats with mono- or polyunsaturated fats & + & B & 129,137 \\
\hline \multicolumn{4}{|l|}{ Lifestyle interventions to increase HDL-C levels } \\
\hline Avoid dietary trans fats & ++ & A & 129,160 \\
\hline Increase habitual physical activity & +++ & A & 151,161 \\
\hline Reduce excessive body weight & ++ & A & 147,148 \\
\hline Reduce dietary carbohydrates and replace them with unsaturated fats & ++ & A & 147,162 \\
\hline Modest consumption in those who take alcohol may be continued & ++ & B & 153 \\
\hline Quit smoking & + & B & 163 \\
\hline
\end{tabular}

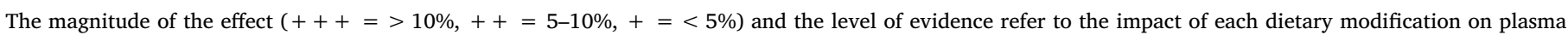
levels of a specific lipoprotein class.

HDL-C = high-density lipoprotein cholesterol; LDL-C = low-density lipoprotein cholesterol; TC = total cholesterol; TG = triglyceride. 
Table 9

Food choices to lower low-density lipoprotein cholesterol and improve the overall lipoprotein profile.

\begin{tabular}{|c|c|c|c|}
\hline & To be preferred & To be used in moderation & To be chosen occasionally in limited amounts \\
\hline Cereals & Wholegrains & Refined bread, rice, and pasta, biscuits, corn flakes & Pastries, muffins, pies, croissants \\
\hline Vegetables & Raw and cooked vegetables & Potatoes & Vegetables prepared in butter or cream \\
\hline Legumes & $\begin{array}{l}\text { Lentils, beans, fava beans, peas, } \\
\text { chickpeas, soybean }\end{array}$ & & \\
\hline Fruit & Fresh or frozen fruit & $\begin{array}{l}\text { Dried fruit, jelly, jam, canned fruit, sorbets, ice } \\
\text { lollies/popsicles, fruit juice }\end{array}$ & \\
\hline Sweets and sweeteners & Non-caloric sweeteners & Sucrose, honey, chocolate, sweets/candies & Cakes, ice creams, fructose, soft drinks \\
\hline Meat and fish & $\begin{array}{l}\text { Lean and oily fish, poultry without } \\
\text { skin }\end{array}$ & $\begin{array}{l}\text { Lean cuts of beef, lamb, pork, and veal, seafood, } \\
\text { shellfish }\end{array}$ & $\begin{array}{l}\text { Sausages, salami, bacon, spare ribs, hot dogs, organ } \\
\text { meats }\end{array}$ \\
\hline Dairy food and eggs & Skimmed milk and yoghurt & $\begin{array}{l}\text { Low-fat milk, low-fat cheese and other milk } \\
\text { products, eggs }\end{array}$ & Regular cheese, cream, whole milk and yoghurt \\
\hline Cooking fat and dressings & $\begin{array}{l}\text { Vinegar, mustard, fat-free } \\
\text { dressings }\end{array}$ & $\begin{array}{l}\text { Olive oil, non-tropical vegetable oils, soft } \\
\text { margarines, salad dressing, mayonnaise, ketchup }\end{array}$ & $\begin{array}{l}\text { Trans fats and hard margarines (better to avoid them), } \\
\text { palm and coconut oils, butter, lard, bacon fat }\end{array}$ \\
\hline Nuts/seeds & & All, unsalted (except coconut) & Coconut \\
\hline Cooking procedures & Grilling, boiling, steaming & Stir-frying, roasting & Frying \\
\hline
\end{tabular}

Secondary goals have also been defined by inference for non-HDL-C and for ApoB; they receive a moderate grading, as they have not been extensively studied in RCTs. The specific goal for non-HDL-C should be $0.8 \mathrm{mmoL} / \mathrm{L}(30 \mathrm{mg} / \mathrm{dL}$ ) higher than the corresponding LDL-C goal; the adjustment of lipid-lowering therapy in accordance with these secondary goals may be considered in patients at very high CV risk after achievement of an LDL-C goal, although the clinical advantages of this approach with respect to outcomes remain to be addressed. When secondary targets are used the recommendations are: (i) non-HDL$\mathrm{C}<2.2 \mathrm{mmoL} / \mathrm{L} \quad(<85 \mathrm{mg} / \mathrm{dL}),<2.6 \mathrm{mmoL} / \mathrm{L}(<100 \mathrm{mg} / \mathrm{dL})$, and $<3.4 \mathrm{mmoL} / \mathrm{L}(<130 \mathrm{mg} / \mathrm{dL})$ in people at very high, high, and moderate CV risk, respectively [121-123]; and (ii) ApoB $<65 \mathrm{mg} / \mathrm{dL}$, $<80 \mathrm{mg} / \mathrm{dL}$, and $<100 \mathrm{mg} / \mathrm{dL}$ in very-high, high, and moderate total CV risk, respectively $[121,123,124]$.

To date, no specific goals for HDL-C or TG levels have been determined in clinical trials, although increases in HDL-C predict atherosclerosis regression, and low HDL-C is associated with excess events and mortality in coronary artery disease (CAD) patients, even at low LDL levels. Clinicians should use clinical judgment when considering further treatment intensification in patients at high or very high total CV risk.

\section{Lifestyle modifications to improve the plasma lipid profile}

The pivotal role of nutrition in the prevention of ASCVD has been extensively reviewed [125-129]. Dietary factors influence the development of CVD either directly or through their action on traditional risk factors, such as plasma lipids, BP, or glucose levels.

Convincing evidence of the causal association between diet and ASCVD risk is, nevertheless, available indirectly from randomized 'metabolic ward' studies showing that high saturated fat intake causes increased LDL-C concentrations, and from cohort studies, genetic epidemiological studies, and randomized trials showing that higher LDL-C levels cause ASCVD.

The lack of concordance between studies is due both to methodological problems (particularly inadequate sample sizes or short study durations) and the difficulties of evaluating the impact of a single dietary factor independently of any other changes in the diet [130]. In fact, as foods are mixtures of different nutrients and other components, it is not appropriate to attribute the health effects of a food to only one of its components. Moreover, if energy intake must be kept constant, eating less of one macronutrient implies necessarily eating more of others. The quality of the replacement (for instance, unsaturated fat vs. highly refined grains) can influence the effect observed, significantly modifying the impact on health of the nutrient replaced. These limitations suggest caution in interpreting the results of RCTs or even meta-analyses of RCTs in relation to the effect of a single dietary change on ASCVD [130].

To overcome, at least in part, these problems, in recent years nutrition research has focused on the relationship between ASCVD on the one hand, and foods and dietary patterns-rather than single nutrients-on the other. Consistent evidence from epidemiological studies indicates that higher consumption of fruit, non-starchy vegetables, nuts, legumes, fish, vegetable oils, yoghurt, and wholegrains, along with a lower intake of red and processed meats, foods higher in refined carbohydrates, and salt, is associated with a lower incidence of CV events [131]. Moreover, it indicates that the replacement of animal fats, including dairy fat, with vegetable sources of fats and polyunsaturated fatty acids (PUFAs) may decrease the risk of CVD [132].

Dietary patterns that have been more extensively evaluated are the Dietary Approaches to Stop Hypertension (DASH) diet-particularly in relation to BP control-and the Mediterranean diet; both have proved to be effective in reducing CV risk factors and, possibly, to contribute to ASCVD prevention [133]. The most relevant difference between the Mediterranean and the DASH diet is the emphasis of the former on extra-virgin olive oil. The Mediterranean diet is associated with a reduced incidence of CV and other non-communicable diseases in epidemiological studies [134,135], and has been proved in RCTs to be effective in reducing CV events in primary and secondary prevention [136]. In particular, the Prevención con Dieta Mediterránea (PREDIMED) trial indicated that participants allocated to a Mediterranean-type diet, supplemented with extra-virgin olive oil or nuts, had a significantly lower (around 30\%) incidence of major CV events compared with those who were on a low-fat diet [137].

In summary, despite the results of PREDIMED and a few other intervention studies with ASCVD endpoints that support a healthy lifestyle for ASCVD prevention, RCTs cannot represent the sole grounds on which dietary recommendations should rely. They also need to be based on the combination of large observational cohort studies and relatively short-term randomized trials having intermediate risk factors (such as blood lipids) as outcomes.

Table 8 summarizes the currently available evidence on the influences of lifestyle changes and functional foods on lipoproteins, indicating the magnitudes of the effects and the levels of evidence in relation to the impacts on the specific lipoprotein class; for the reasons outlined above, the levels of evidence are not based on RCTs with ASCVD endpoints. Moreover, within the Guidelines on the management of dyslipidaemias, information on the potential to improve plasma lipoprotein profiles by dietary means is clinically relevant, even in the absence of a clear demonstration of CV benefits. 


\subsection{Influence of lifestyle on total cholesterol and low-density lipoprotein cholesterol levels}

Table 9 summarizes the possible choices of foods to lower TC and LDL-C levels. Given the cultural diversity of the European populations, they should be translated into practical behaviours, considering local habits and socio-economic factors.

\subsection{Influence of lifestyle on triglyceride levels}

Weight reduction improves insulin sensitivity and decreases TG levels. Regular physical exercise reduces plasma TG levels over and above the effect of weight reduction [151,168,169]. Alcohol intake has a major impact on TG levels, particularly in individuals with HTG $[153,170]$. The detrimental effects of a high-carbohydrate diet on TGs occur mainly when refined carbohydrate-rich foods are consumed, while they are much less prominent if the diet is based largely on fibrerich, low-glycaemic index foods. This applies particularly to people with DM or MetS [171,172].

Habitual consumption of significant amounts ( $>10 \%$ energy) of dietary fructose contributes to TG elevation, particularly in people with HTG or abdominal obesity. These effects are dose-dependent; with a habitual fructose consumption between 15 and 20\% of total energy intake, plasma TG increases by as much as $30-40 \%$. Sucrose, a disaccharide containing glucose and fructose, represents an important source of fructose in the diet $[159,173,174]$.

\subsection{Influence of lifestyle on high-density lipoprotein cholesterol levels}

Weight reduction increases HDL-C levels; a $0.01 \mathrm{mmoL} / \mathrm{L}(0.4 \mathrm{mg} /$ $\mathrm{dL}$ ) increase is observed for every kilogram decrease in body weight when weight reduction has stabilized. Aerobic physical activity, such as 25-30 km of brisk walking per week (or any equivalent activity), may increase HDL-C levels by $0.08-0.15 \mathrm{mmoL} / \mathrm{L}(3.1-6 \mathrm{mg} / \mathrm{dL})$ [169]. Smoking cessation may also contribute to HDL-C elevation, provided that weight gain is prevented [163].

\subsection{Lifestyle recommendations to improve the plasma lipid profile}

LDL-C lowering represents the primary target for reducing CV risk and therefore deserves special emphasis in the evaluation of lifestyle measures. The diet recommended to the general population, and particularly to people at increased CV risk, may also be able to modify plasma TG and HDL-C levels (Table 9). This section focuses on dietary and other lifestyle factors that may be implemented to improve the overall lipoprotein profile.

\subsubsection{Body weight and physical activity}

Since overweight, obesity, and-in particular-abdominal adiposity often contribute to dyslipidaemia, caloric intake should be reduced and energy expenditure increased in those with excessive weight and/or abdominal adiposity.

In the case of excess weight, body weight reduction, even if modest (5-10\% of basal body weight), improves lipid abnormalities and favourably affects the other $\mathrm{CV}$ risk factors often present in dyslipidaemic individuals [148]. While the beneficial effects of weight reduction on metabolic and surrogate markers have been demonstrated, the benefits of weight loss on mortality and CV outcome are less clear [175].

Weight reduction can be achieved by decreasing the consumption of energy-dense foods, inducing a caloric deficit of 300-500 kcaL/day. The intervention should combine diet and exercise; this approach also leads to the greatest improvement in physical performance and quality of life, and mitigates reductions in muscle and bone mass, particularly in older people [176]. It is always appropriate to advise people with dyslipidaemia to engage in regular physical exercise of moderate intensity for $\geq 30 \mathrm{~min} /$ day, even if they are not overweight [168].

\subsubsection{Dietary fat}

Avoiding any consumption of trans fat is a key measure of the dietary prevention of CVD. The trans fatty acids produced in the partial hydrogenation of vegetable oils account for $80 \%$ of total intake. Thanks to efforts made in different parts of the world, the intake of trans fatty acids has decreased substantially over the past 10-15 years.

As for saturated fat, its consumption should be $<10 \%$ of the total caloric intake and should be further reduced ( $<7 \%$ of energy) in the presence of hypercholesterolaemia. For most individuals, a wide range of total fat intakes is acceptable, and will depend upon individual preferences and characteristics. However, fat intakes $>35-40 \%$ of calories are generally associated with increased intakes of both saturated fat and calories. Conversely, low intakes of fats and oils increase the risk of inadequate intakes of vitamin $\mathrm{E}$ and of essential fatty acids, and may contribute to a reduction of HDL-C [164].

Fat intake should predominantly come from sources of monounsaturated fatty acids, including both n-6 and n-3 PUFAs. Not enough data are available to make a recommendation regarding the optimal n3:n-6 fatty acid ratio $[177,178]$. The cholesterol intake in the diet should be reduced $(<300 \mathrm{mg} /$ day), particularly in people with high plasma cholesterol levels.

\subsubsection{Dietary carbohydrate and fibre}

Dietary carbohydrate has a 'neutral' effect on LDL-C, although excessive consumption is represented by untoward effects on plasma TGs and HDL-C levels [164]. Dietary fibre (particularly of the soluble type)-which is present in legumes, fruits, vegetables, and wholegrain cereals (e.g. oats and barley)—has a hypocholesterolaemic effect and represents a good dietary substitute for saturated fat to maximize the effects of the diet on LDL-C levels, and to minimize the untoward effects of a high-carbohydrate diet on other lipoproteins [140,179].

Carbohydrate intake should range between 45 and 55\% of total energy intake, since both higher and lower percentages of carbohydrate diets are associated with increased mortality $[180,181]$. A fat-modified diet that provides $25-40 \mathrm{~g}$ per day of total dietary fibre, including $\geq 7-13 \mathrm{~g}$ of soluble fibre, is well tolerated, effective, and recommended for plasma lipid control; conversely, there is no justification for the recommendation of very low-carbohydrate diets [182].

Intake of added sugar should not exceed $10 \%$ of total energy (in addition to the amount present in natural foods such as fruits and dairy products); more restrictive advice concerning sugars may be useful for those needing to lose weight or with high plasma TG values, MetS, or DM. Soft drinks should be used with moderation by the general population, and should be drastically limited in those individuals with elevated TG values or visceral adiposity $[158,159,174]$. The Prospective Urban Rural Epidemiology (PURE) study was a large, epidemiological cohort study of 135335 individuals enrolled in 18 countries with food frequency questionnaires recorded. Total fat and types of fat were not associated with CVD, MI, or CVD mortality, whereas saturated fat had an inverse association with stroke [181]. However, a meta-analysis of epidemiological studies including the PURE study showed a U-shaped relationship between carbohydrate intake and mortality: diets associated with the highest mortality rate had carbohydrate intakes $>70 \%$ and $<40 \%$ of energy, with minimal risk observed when carbohydrate intake was between 45 and 55\% of total energy intake [180].

\subsubsection{Alcohol}

Moderate alcohol consumption [ $\leq 10 \mathrm{~g} /$ day (1 unit) for men and women] is acceptable for those who drink alcoholic beverages, if TG levels are not elevated [183,184].

\subsubsection{Smoking}

Smoking cessation has clear benefits regarding overall CV risk, and specifically on HDL-C levels [163]. 


\subsection{Dietary supplements and functional foods for the treatment of dyslipidaemias}

Nutritional evaluation of functional foods includes not only the search for clinical evidence of beneficial effects relevant to improved health or the reduction of disease risk, but also the demonstration of good tolerability. Overall, the available evidence on functional foods so far identified in this field is incomplete; the major gap is an absence of diet-based intervention trials of enough duration to be relevant for the natural history of dyslipidaemia and CVD.

\subsubsection{Phytosterols}

The principal phytosterols are sitosterol, campesterol, and stigmasterol; they occur naturally in vegetable oils and in smaller amounts in vegetables, fresh fruits, nuts, grains, and legumes. The dietary intake of plant sterols ranges between an average of $250 \mathrm{mg} /$ day in Northern Europe to $\sim 500 \mathrm{mg} /$ day in Mediterranean countries. Phytosterols compete with cholesterol for intestinal absorption, thereby modulating TC levels.

Daily consumption of $2 \mathrm{~g}$ of phytosterols can effectively lower TC and LDL-C levels by $7-10 \%$ in humans (with a certain degree of heterogeneity among individuals), while it has little or no effect on HDL-C and TG levels [143]. However, to date no studies have been performed on the subsequent effect on CVD. Based on LDL-C lowering and the absence of adverse signals, functional foods with plant sterols/stanols ( $\geq 2 \mathrm{~g} /$ day with the main meal) may be considered: (i) in individuals with high cholesterol levels at intermediate or low global CV risk who do not qualify for pharmacotherapy; (ii) as an adjunct to pharmacological therapy in high- and very-high-risk patients who fail to achieve LDL-C goals on statins or could not be treated with statins; and (iii) in adults and children (aged $>6$ years) with $\mathrm{FH}$, in line with current guidance [142].

\subsubsection{Monacolin and red yeast rice}

Red yeast rice (RYR) is a source of fermented pigment that has been used in China as a food colorant and flavour enhancer for centuries. Hypocholesterolaemic effects of RYR are related to a statin-like mechanism-inhibition of hydroxymethylglutaryl-coenzyme A (HMG$\mathrm{CoA}$ ) reductase-of monacolins, which represent the bioactive ingredient. Different commercial preparations of RYR have different concentrations of monacolins, and lower TC and LDL-C levels to variable extents, but the consumer is not able to make that distinction $[144,185]$. Moreover, the long-term safety of the regular consumption of these products has not been fully documented and safety issues due to the possible presence of contaminants in some preparations have been raised. Side effects like those observed with statins have also been reported.

In the only available RCT in patients with ASCVD, a partially purified extract of RYR reduced recurrent events by 45\% [146]. A clinically relevant hypocholesterolaemic effect (up to a $20 \%$ reduction) has been observed with RYR preparations providing an o.d. [once daily (omni die)] dose of 5-10 mg monacolin K [145]. Nutraceuticals containing purified RYR may be considered in people with elevated plasma cholesterol concentrations who do not qualify for treatment with statins in view of their global CV risk. However, there is a clear need for better regulation of RYR supplements. Information regarding the precise composition of these products, the quantities of their components, and their purity should be implemented [185].

\subsubsection{Dietary fibre}

Available evidence consistently demonstrates a TC- and LDL-Clowering effect of $\beta$-glucan, a viscous fibre from oat and barley. Foods enriched with these fibres or supplements are well tolerated, effective, and recommended for LDL-C lowering [186]. However, the dosage needed to achieve a clinically relevant reduction in levels of LDL-C of $3-5 \%$ varies from 3 to $10 \mathrm{~g}$ per day according to the specific type of fibre [187].

\subsubsection{Soy}

The cholesterol-lowering effect of soy is generally attributed to its isoflavone and phytoestrogen content, which decreases progressively with the increasing degree of soybean processing. Soy protein has also been indicated as being able to induce a modest LDL-C-lowering effect when replacing animal protein foods. However, this was not confirmed when changes in other dietary components were taken into account $[187,188]$.

\subsubsection{Policosanol and berberine}

Policosanol is a natural mixture of long-chain aliphatic alcohols extracted primarily from sugarcane wax [189]. Studies show that policosanol from sugarcane, rice or wheat germ has no significant effect on LDL-C, HDL-C, TG, ApoB, Lp(a), homocysteine, high-sensitivity Creactive protein, fibrinogen, or blood coagulation factor levels [190].

As for berberine, a recent meta-analysis evaluated its effects on plasma lipids in humans [191]. The comparative evaluation of berberine and lifestyle intervention or placebo indicated that in the berberine group, LDL-C and plasma TG levels were more effectively reduced than in the control group. However, due to the lack of highquality randomized clinical trials, the efficacy of berberine for treating dyslipidaemia needs to be further validated. Moreover, the bioavailability of the different berberine preparations is a matter of debate [187].

\subsection{6. n-3 unsaturated fatty acids}

Observational evidence indicates that consumption of fish (at least twice a week) and vegetable foods rich in $n-3$ fatty acids ( $\alpha$-linoleic acid is present in walnuts, some vegetables, and some seed oils) is associated with lower risk of $\mathrm{CV}$ death and stroke, but has no major effects on plasma lipoprotein metabolism [178,192]. Pharmacological doses of long-chain n-3 fatty acids (2-3 g/day) reduce TG levels by about $30 \%$ and also reduce the post-prandial lipaemic response, but a higher dosage may increase LDL-C levels. $\alpha$-Linolenic acid is less effective at altering TG levels [156,193]. Recently, a significantly lower risk of ischaemic events, including CV death, was observed in patients with elevated TG levels despite the use of statins treated with $2 \mathrm{~g}$ of icosapent ethyl b.i.d. [twice a day] [194].

Other features of a healthy diet contributing to CVD prevention are presented in the Supplementary Data.

\section{Drugs for treatment of dyslipidaemias}

\subsection{Statins}

\subsubsection{Mechanism of action}

Statins reduce the synthesis of cholesterol in the liver by competitively inhibiting the enzyme HMG-CoA reductase, the rate-limiting step in cholesterol biosynthesis. The reduction in intracellular cholesterol promotes increased LDL receptor (LDLR) expression at the surface of the hepatocytes, which in turn results in increased uptake of LDL from the blood, and decreased plasma concentrations of LDL- and other ApoB-containing lipoproteins, including TG-rich particles.

\subsubsection{Effects on lipids}

8.1.2.1. Low-density lipoprotein cholesterol. The degree of LDL-C reduction is dose-dependent and varies between the different statins. A high-intensity regimen is defined as the dose of a statin that, on average, reduces LDL-C by $\geq 50 \%$; moderate-intensity therapy is defined as the dose expected to reduce LDL-C by $30-50 \%$. Notably, there is considerable interindividual variation in LDL-C reduction with the same dose of drug [31]. Poor responses to statin treatment in clinical studies are to some extent caused by poor compliance, but may also be explained by genetic backgrounds [195,196]. Interindividual 
variations in statin responses warrant monitoring of responses on initiation of therapy.

Among patients who cannot tolerate the recommended intensity of a statin because of adverse effects or those who do not reach their goal, the addition of a non-statin lipid-modifying agent to a maximally tolerated statin is recommended [197,198].

8.1.2.2. Triglycerides. Statins usually reduce TG levels by $10-20 \%$ from baseline values [199]. More potent statins (atorvastatin, rosuvastatin, and pitavastatin) demonstrate robust lowering of TG levels, especially at high doses and in patients with elevated TGs (HTG), in whom the absolute risk, and therefore the absolute risk reduction, is larger.

The mechanism of the TG-lowering effect has not been fully elucidated, but it seems to be partly independent of the LDLR pathway. It may involve the upregulation of VLDL uptake by hepatocytes, as well as a reduction of the production rate of VLDLs; these effects seem to be dependent on pre-treatment VLDL concentrations [200].

8.1.2.3. High-density lipoprotein cholesterol. In a meta-analysis [201], elevations in HDL-C levels varied with dose among the respective statins; such elevations ranged from 1 to $10 \%$. However, given the marked statin-mediated decrement in atherogenic ApoB-containing lipoproteins, the extent to which the very modest effect on HDL-C levels might contribute to the overall observed reductions in CV risk consistently observed in statin intervention trials cannot reliably be disentangled.

8.1.2.4. Lipoprotein(a). Statins only marginally affect Lp(a) plasma levels. Previous studies have reported either no effect on or an increase of Lp(a) levels after statin treatment [202,203]. The mechanisms by which statins raise oxidized phospholipids on Lp(a) require further investigation.

\subsubsection{Other effects of statins}

Although reduction of LDL-C levels is the major effect of statins, a number of other, potentially important effects have been suggested (pleiotropic effects of statins) [204,205]. Among such effects that are potentially relevant for the prevention of CVD are the anti-inflammatory and antioxidant effects of statin treatment. These effects have been shown in vitro and in experimental systems, but their clinical relevance remains unproven $[18,206]$.

8.1.3.1. Effect on cardiovascular morbidity and mortality. A large number of meta-analyses have been performed to analyse the effects of statins in populations and in subgroups [34-36,38,51,207-214]. In the Cholesterol Treatment Trialists (CTT) meta-analysis of individual participant data (IPD) from $>170000$ participants in 26 RCTs of a statin vs. control or a more vs. less intensive statin regimen [34], for each $1 \mathrm{mmoL} / \mathrm{L}$ reduction in LDL-C, statin/more statin reduced major vascular events (MI, CAD death, or any stroke or coronary revascularization) by $\sim 22 \%$, major coronary events by $23 \%$, CAD death by $20 \%$, total stroke by $17 \%$, and total mortality by $10 \%$ over 5 years. The proportional effects (per mmol/L reduction in LDL-C) on major vascular events were similar in all subgroups examined, so the absolute risk reduction was proportional to the absolute baseline risk. The relative benefits were half as large in the first year as compared with subsequent years. There was no increased risk for any non-CV cause of death, including cancer, in those allocated statins. The absolute benefit from statin treatment was lower in people in primary prevention, who are typically at lower risk [36,38,214,215]. In the CTT meta-analysis of treatment in people with a low-risk of vascular disease [36], the relative risk reduction of major vascular events per $\mathrm{mmol} / \mathrm{L}$ reduction in LDL-C was at least as large in low-risk individuals (i.e. in primary prevention). In those without a history of vascular disease, statin therapy reduced the risk of all-cause mortality by $9 \%$ per $\mathrm{mmol} / \mathrm{L}$ reduction in LDL cholesterol. Similar results were reported in a
Cochrane review in 2013 [213]. The West of Scotland Coronary Prevention Study (WOSCOPS) data were recently reanalysed, and demonstrated that even people without DM and a 10 year predicted ASCVD risk of $<7.5 \%$ benefit from statin treatment. There was also a legacy effect with a mortality benefit of $18 \%$ in all-cause death over 20 years [216]. Statins are effective for the prevention of ASCVD in the elderly, including those aged $>75$ years [217]. Statins are not effective in a few specific groups, notably those with heart failure (HF) or patients receiving haemodialysis [214,218-222].

Current available evidence from meta-analyses suggests that the clinical benefit of statin treatment is largely a class effect, driven by the absolute LDL-C reduction; therefore, the type of statin used should reflect the treatment goals for a given patient.

The following scheme may be proposed.

- Evaluate the total CV risk of the individual.

- Determine the treatment goals (depending on current risk).

- Involve the patient in decisions on CV risk management.

- Choose a statin regimen and, where necessary, additional treatments (e.g. ezetimibe or PCSK9 inhibitors) that can meet the treatment goals (per cent and absolute value).

- Response to statin treatment is variable, therefore uptitration of the statin dose may be required before additional LDL-lowering treatments are started.

These are general criteria for the choice of drug. Factors such as the clinical condition of the patient, concomitant medications, drug tolerability, local treatment tradition, and drug cost will play major roles in determining the final choice of drug and dose.

Furthermore, the effects of statins on a number of other clinical conditions have been evaluated. For cancer, a meta-analysis of IPD from randomized trials has shown that statins do not have any significant effect on cancer, at least over a period of $\sim 5$ years [223]. Other conditions, such as dementia [224], hepatic steatosis [225], venous thromboembolism [226], atrial fibrillation [227,228], and polycystic ovary syndrome [229] have also been studied, and no effect of statins on these conditions has been reliably demonstrated.

The suggested effect on Alzheimer's disease was recently reviewed in a Cochrane analysis reporting no conclusive effect from statins [230]. Furthermore, neurocognitive functions were extensively investigated in the Evaluating PCSK9 Binding Antibody Influence on Cognitive Health in High Cardiovascular Risk Subjects (EBBINGHAUS) study [231] and no excess risk was observed among patients on a statin regimen randomized to a PCSK9 mAb.

\subsubsection{Adverse effects and interactions of statins}

Statins differ in their absorption, bioavailability, plasma protein binding, excretion, and lipophilicity. Evening administration is usually recommended. Lovastatin and simvastatin are prodrugs, whereas the other available statins are administered in their active form. Their bioavailability is relatively low, owing to a first-pass effect in the liver, and many statins undergo significant hepatic metabolism via cytochrome P450 (CYP) isoenzymes, except pravastatin, rosuvastatin, and pitavastatin. These enzymes are expressed mainly in the liver and gut wall. Although statins are generally very well tolerated, they do have some specific adverse effects on muscle, glucose haemostasis, and haemorrhagic stroke. However, there is also widespread misinformation about potential adverse effects, as reviewed recently [232,233].

8.1.4.1. Adverse effects on muscle. Myopathy is the most clinically relevant adverse effect of statins. Among the risk factors for myopathy, it is particularly important that interaction with concomitant drug therapy is considered (see below). Rhabdomyolysis is the most severe form of statin-induced muscle damage, characterized by severe muscular pain, muscle necrosis, and myoglobinuria potentially leading to renal failure and death. In rhabdomyolysis, 
creatine kinase (CK) levels are elevated by $\geq 10$ times, and often $\geq 40$ times, the upper limit of normal (ULN) [234]. The frequency of rhabdomyolysis has been estimated to represent 1-3 cases/100 000 patient-years [235]. Patients taking statin therapy frequently report muscle symptoms [so-called 'statin-associated muscle symptoms' (SAMS)], and in non-randomized, observational studies, statins are associated with muscular pain and tenderness (myalgia) without CK elevation or major functional loss, with the reported frequency of SAMS in such studies varying between 10 and 15\% among statin-treated individuals [236-238]. However, in part because individuals in observational studies are not blind to the treatment they are receiving, such studies are unreliable when used to assess the adverse effects of statins [233]. In contrast, in blinded randomized trials of statins vs. placebo there is no, or only a slightly, increased frequency of muscle symptoms in statin-allocated groups [239,240]. The AngloScandinavian Cardiac Outcomes Trial - Lipid-Lowering Arm (ASCOTLLA) study addressed this issue by comparing the incidence of four different adverse events, including muscle-related symptoms, during both the blinded, placebo-controlled trial and its open-label extension study [238]. They concluded that a nocebo effect (i.e. one caused by negative expectations) may partly explain the higher frequency of SAMS in observational studies compared with in trials. Suggested practical management of muscular symptoms is shown in Supplementary Fig. 6 [198,234,241]. Several studies have shown a considerable LDL-C-lowering effect of alternative dosing, such as every other day or twice a week, with atorvastatin or rosuvastatin [242]. Although no clinical endpoint trials are available, this strategy should be considered in high-risk patients in whom statin treatment with daily doses is not possible.

8.1.4.2. Adverse effects on the liver. The activity of alanine aminotransferase (ALT) in plasma is commonly used to assess hepatocellular damage. Mild elevation of ALT occurs in $0.5-2.0 \%$ of patients on statin treatment, more commonly with potent statins or high doses. The common definition of clinically relevant ALT elevation has been an increase of three times the ULN on two consecutive occasions. Mild elevation of ALT has not been shown to be associated with true hepatotoxicity or changes in liver function. Progression to liver failure is exceedingly rare, therefore routine monitoring of ALT during statin treatment is no longer recommended [243]. Patients with mild ALT elevation due to steatosis have been studied during statin treatment and there is no indication that statins cause any worsening of liver disease [244-246].

8.1.4.3. Increased risk of new-onset diabetes mellitus. Patients on statin treatment have been shown to exhibit an increased risk of dysglycaemia and development of type 2 diabetes mellitus (T2DM). Several studies have shown that this is a consistent, dose-related effect [232]. A minor, not clinically relevant elevation of glycated haemoglobin (HbA1c) has also been observed. The number needed to cause one case of diabetes has been estimated as 255 over 4 years of statin treatment [247]. However, the risk is higher with the more potent statins at high doses [248], and is also higher in the elderly, and in the presence of other risk factors for diabetes such as overweight or insulin resistance [249]. Overall, the absolute reduction in the risk of CVD in high-risk patients clearly outweighs the possible adverse effects of a small increase in the incidence of diabetes [233]. This effect is probably related to the mechanism of action of statins, as Mendelian randomization studies have confirmed the increased risk of DM in individuals with HMG-CoA reductase polymorphisms that reduce cholesterol synthesis [250].

8.1.4.4. Increased risk of haemorrhagic stroke. In observational studies, TC is negatively associated with haemorrhagic stroke, and in the CTT meta-analysis, there was a $21 \%$ [ $95 \%$ confidence interval (CI) $5-41 \%$; $P=0.01]$ relative increase per $\mathrm{mmol} / \mathrm{L}$ lower LDL cholesterol in haemorrhagic stroke [34,251,252]. However, other meta-analyses
Table 10

Drugs potentially interacting with statins metabolized by cytochrome P450 3A4 leading to increased risk of myopathy and rhabdomyolysis.

\begin{tabular}{lll}
\hline Anti-infective agents & Calcium antagonists & Other \\
\hline Itraconazole & Verapamil & Ciclosporin \\
Ketoconazole & Diltiazem & Danazol \\
Posaconazole & Amlodipine & Amiodarone \\
Erythromycin & & Ranolazine \\
Clarithromycin & & Grapefruit juice \\
Telithromycin & Nefazodone \\
HIV protease inhibitors & & Gemfibrozil \\
\hline
\end{tabular}

Adapted from Egan and Colman [257], and Wiklund et al. [258].

HIV = human immunodeficiency virus.

have yielded conflicting findings and there is a need for further exploration of the risk of haemorrhagic stroke in particular types of patients. Note, however, that the overall benefit on other stroke subtypes greatly outweighs this small (and uncertain) hazard [34,36].

8.1.4.5. Adverse effects on kidney function. There is no clear evidence that statins have a clinically significant beneficial or adverse effect on renal function [253]. An increased frequency of proteinuria has been reported for all statins, but has been analysed in more detail for rosuvastatin. With a dose of $80 \mathrm{mg}$, a frequency of $12 \%$ was reported. With the approved doses of $<40 \mathrm{mg}$, the frequency is much lower and in line with the frequency for other statins. The proteinuria induced by statins is of tubular origin, usually transitory, and is believed to be due to reduced tubular reabsorption and not to glomerular dysfunction [254,255]. In clinical trials, the frequency of proteinuria is generally low and, in most cases, is not higher than for placebo [256].

8.1.4.6. Interactions. A number of important drug interactions with statins have been described that may increase the risk of adverse effects. Inhibitors and inducers of enzymatic pathways involved in statin metabolism are summarized in Table 10. All currently available statins-except pravastatin, rosuvastatin, and pitavastatin-undergo major hepatic metabolism via the CYPs. These isoenzymes are mainly expressed in the liver and intestine. Pravastatin does not undergo metabolism through the CYP system, but is metabolized by sulfation and conjugation. CYP3A4 isoenzymes are the most abundant, but other isoenzymes such as CYP2C8, CYP2C9, CYP2C19, and CYP2D6 are frequently involved in the metabolism of statins. Thus, other pharmacological substrates of these CYPs may interfere with statin metabolism. Conversely, statin therapy may interfere with the catabolism of other drugs that are metabolized by the same enzymatic system.

Combination of statins with gemfibrozil enhances the risk of myopathy, and its association with statins must be avoided. There is no or very little increased risk for myopathy when combining statins with other fibrates, such as fenofibrate, bezafibrate, or ciprofibrate $[259,260]$.

\subsection{Cholesterol absorption inhibitors}

\subsubsection{Mechanism of action}

Ezetimibe inhibits intestinal uptake of dietary and biliary cholesterol at the level of the brush border of the intestine [by interacting with the Niemann-Pick C1-like protein 1 (NPC1L1)] without affecting the absorption of fat-soluble nutrients. By inhibiting cholesterol absorption, ezetimibe reduces the amount of cholesterol delivered to the liver. In response to reduced cholesterol delivery, the liver reacts by upregulating LDLR expression, which in turn leads to increased clearance of LDL from the blood. 


\subsubsection{Effects on lipids}

In clinical studies, ezetimibe in monotherapy at $10 \mathrm{mg} /$ day reduces LDL-C in hypercholesterolaemic patients by $15-22 \%$ with relatively high interindividual variation [261]. A meta-analysis of RCTs that included over 2700 people showed an $18.5 \%$ reduction in LDL-C as compared with placebo [262]. In addition, there was a significant $3 \%$ increase in HDL-C, a significant $8 \%$ reduction in TGs, and a $13 \%$ reduction in TC with ezetimibe as compared with placebo.

Ezetimibe added to ongoing statin therapy reduces LDL-C levels by an additional $21-27 \%$ compared with placebo in patients with hypercholesterolaemia with or without established CHD. In statin-naïve patients, ezetimibe and statin combination therapy has resulted in around a $15 \%$ greater reduction in LDL-C when compared with the same statins and doses in monotherapy. In other studies, this combination has also significantly improved reductions in LDL-C levels when compared with doubling of the statin dose (13-20\%), and after switching from statin monotherapy to ezetimibe and statin combination therapy (11-15\%) [263].

Co-administration of ezetimibe and bile acid sequestrants (colesevelam, colestipol, or cholestyramine) has been reported to result in an additional reduction of LDL-C levels by $10-20 \%$ when compared with the stable bile acid sequestrant regimen alone [264]. Co-administration of ezetimibe with PCSK9 inhibitors also results in an additional effect [265].

\subsubsection{Effect on cardiovascular morbidity and mortality}

The efficacy of ezetimibe in association with simvastatin has been addressed in people with aortic stenosis in the Simvastatin and Ezetimibe in Aortic Stenosis (SEAS) trial [266], and in patients with CKD in the Study of Heart and Renal Protection (SHARP) trial [222]. In both the SEAS and SHARP trials, a reduction in CV events was demonstrated in the simvastatin-ezetimibe arm vs. placebo [266,267].

In the Improved Reduction of Outcomes: Vytorin Efficacy International Trial (IMPROVE-IT), ezetimibe was added to simvastatin (40 mg) in patients after acute coronary syndrome (ACS) [33]. A total of 18144 patients were randomized to statin or statin plus ezetimibe, and 5314 patients over 7 years experienced a CV event; 170 fewer events (32.7 vs. $34.7 \%)$ were recorded in the group taking simvastatin plus ezetimibe $(P=0.016)$. The average LDL-C during the study was $1.8 \mathrm{mmoL} / \mathrm{L}(70 \mathrm{mg} / \mathrm{dL})$ in the simvastatin group and $1.4 \mathrm{mmoL} / \mathrm{L}(55$ $\mathrm{mg} / \mathrm{dL}$ ) in patients taking ezetimibe plus simvastatin. Also, ischaemic stroke was reduced by $21 \%$ in this trial $(P=0.008)$. There was no evidence of harm caused by ezetimibe or the further LDL-C reduction. In this group of patients already treated with statins to reach goals, the absolute CV benefit from added ezetimibe was small, although significant and in line with the CTT expectations [268]. Therefore, the study supports the proposition that LDL-C lowering by means other than statins is beneficial and safe. The beneficial effect of ezetimibe is also supported by genetic studies of mutations in NPC1L1; naturally occurring mutations that inactivate the protein were found to be associated with reduced plasma LDL-C and reduced risk for CAD $[55,269,270]$.

Taken together with other studies [271], IMPROVE-IT supports the proposal that ezetimibe should be used as second-line therapy in association with statins when the therapeutic goal is not achieved at the maximal tolerated statin dose, or in cases where a statin cannot be prescribed [272,273].

\subsubsection{Adverse effects and interactions}

Ezetimibe is rapidly absorbed and extensively metabolized to pharmacologically active ezetimibe glucuronide. The recommended dose of ezetimibe of $10 \mathrm{mg}$ /day can be administered in the morning or evening irrespective of food intake. There are no clinically significant effects of age, sex, or race on ezetimibe pharmacokinetics, and no dosage adjustment is necessary in patients with mild hepatic impairment or mild-to-severe renal insufficiency. Life-threatening liver failure with ezetimibe as monotherapy or in combination with statins is extremely rare. The addition of ezetimibe to statin therapy does not appear to increase the incidence of elevated CK levels beyond what is noted with statin treatment alone [261].

\subsection{Bile acid sequestrants}

\subsubsection{Mechanism of action}

Bile acids are synthesized in the liver from cholesterol and are released into the intestinal lumen, but most of the bile acid is returned to the liver from the terminal ileum via active absorption. The two older bile acid sequestrants, cholestyramine and colestipol, are both bile acidbinding exchange resins. The synthetic drug colesevelam is also available in some countries. As bile acid sequestrants are not systemically absorbed or altered by digestive enzymes, the beneficial clinical effects are indirect. By binding the bile acids, the drugs prevent the reabsorption of both the drug and cholesterol into the blood, and thereby remove a large portion of the bile acids from the enterohepatic circulation. The liver, depleted of bile, synthesizes more from hepatic cholesterol, therefore increasing the hepatic demand for cholesterol and increasing LDLR expression, which results in a decrease of circulating LDL.

\subsubsection{Effects on lipids}

At the top daily dose of $24 \mathrm{~g}$ of cholestyramine, $20 \mathrm{~g}$ of colestipol, or $4.5 \mathrm{~g}$ of colesevelam, a reduction in LDL-C of $18-25 \%$ has been observed. No major effect on HDL-C has been reported, while TGs may increase in some predisposed patients [274]. Colesevelam can also reduce glucose levels in hyperglycaemic patients [275].

\subsubsection{Effect on cardiovascular morbidity and mortality}

In clinical trials, bile acid sequestrants have contributed greatly to the demonstration of the efficacy of LDL-C lowering in reducing CV events in hypercholesterolaemic people, with a benefit proportional to the degree of LDL-C lowering. Of note, these studies were performed before many of the modern treatment options were available [276-278].

\subsubsection{Adverse effects and interactions}

Gastrointestinal (GI) adverse effects (most commonly flatulence, constipation, dyspepsia, and nausea) are often present with these drugs, even at low doses, which limits their practical use. These adverse effects can be attenuated by beginning treatment at low doses and ingesting ample fluid with the drug. The dose should be increased gradually. Reduced absorption of fat-soluble vitamins has been reported. Furthermore, these drugs may increase circulating TG levels in certain patients.

Bile acid sequestrants have major drug interactions with several commonly prescribed drugs, and must therefore be administered either $4 \mathrm{~h}$ before or $1 \mathrm{~h}$ after other drugs. Colesevelam is better tolerated and has fewer interactions with other drugs, and can be taken together with statins and several other drugs [279].

\subsection{Proprotein convertase subtilisin/kexin type 9 inhibitors}

\subsubsection{Mechanism of action}

Recently, a new class of drugs, PCSK9 inhibitors, has become available that targets a protein (PCSK9) involved in the control of the LDLR [280]. Elevated concentration or function of this protein in plasma reduces LDLR expression by promoting, upon binding, LDLR lysosomal catabolism and a subsequent increase in plasma LDL concentrations, while lower concentration or function of PCSK9 is related to lower plasma LDL-C levels [281]. Therapeutic strategies have been developed mainly using mAbs; the mechanism of action relates to the reduction of the plasma level of PCSK9, which in turn is not available to bind the LDLR. Since this interaction triggers the intracellular 
degradation of the LDLR, lower levels of circulating PCSK9 will result in increased expression of LDLRs at the cell surface and therefore in a reduction of circulating LDL-C levels [281]. Currently, the only approved PCSK9 inhibitors are two fully human mAbs, alirocumab and evolocumab. Statin treatment increases circulating PCSK9 serum levels [282], and thus the best effect of these mAbs has been demonstrated in combination with statins.

\subsubsection{Effects on lipids}

8.4.2.1. Low-density lipoprotein cholesterol. In clinical trials, alirocumab and evolocumab-either alone or in combination with statins, and/or other lipid-lowering therapies-have been shown to significantly reduce LDL-C levels on average by $60 \%$, depending on dose. The efficacy appears to be largely independent of any background therapy. In combination with high-intensity or maximally tolerated statins, alirocumab and evolocumab reduced LDL-C by $46-73 \%$ more than placebo, and by $30 \%$ more than ezetimibe. Among patients in whom statins cannot be prescribed, PCSK9 inhibition reduced LDL-C when administered in combination with ezetimibe [283]. Both alirocumab and evolocumab have also been shown to effectively lower LDL-C levels in patients who are at high CV risk, including those with DM [284].

Given the mechanism of action, these drugs are effective at reducing LDL-C in all patients capable of expressing LDLR in the liver. Therefore, this pharmacological approach is effective in the vast majority of patients, including those with $\mathrm{HeFH}$ and, albeit to a lower level, those with HoFH with residual LDLR expression. Receptor-deficient HoFH responds poorly to the therapy [285].

8.4.2.2. Triglycerides and high-density lipoprotein cholesterol. These highly efficacious LDL-lowering agents also lower TG levels, and increase those of HDL-C and ApoA-I as a function of the dosing regimen. In phase II trials, evolocumab lowered TG levels by $26 \%$, and raised HDL-C and ApoA-I by 9 and 4\%, respectively; similar findings have been reported for alirocumab [286,287]. However, the TG effect must be confirmed in populations with higher starting plasma TG levels.

8.4.2.3. Lipoprotein(a). In contrast to statins, inhibiting PCSK9 with mAbs also reduces Lp(a) plasma levels. Pooled results of phase II trials have shown that treatment leads to an $\mathrm{Lp}$ (a) reduction of about $30-40 \%$ $[288,289]$. While recent investigations have attempted to unravel the mechanism, it remains unclear. However, it appears to be distinct from that of statins, which also enhance LDLR function but do not lower circulating $\mathrm{Lp}$ (a) levels in humans. The relative contribution of this effect to the reduction of risk remains to be addressed in appropriately designed studies.

\subsubsection{Effect on cardiovascular morbidity and mortality}

Early preliminary data from phase III trials suggests a reduction of CV events in line with the LDL-C reduction achieved [286,290,291].

Recently, two major studies were completed: the Further Cardiovascular Outcomes Research with PCSK9 Inhibition in Subjects with Elevated Risk (FOURIER) and the Evaluation of Cardiovascular Outcomes After an Acute Coronary Syndrome During Treatment With Alirocumab (ODYSSEY Outcomes) trials [119,120]. The designs of the trials were similar with regard to the settings of secondary prevention and background therapy; however, the populations enrolled had either stable CHD, peripheral arterial disease (PAD), or stroke; or a recent (median 2.6 months) ACS, respectively. The relative benefit ranged from 15 to $20 \%$ reductions in the risk of the primary endpoints. Both studies had relatively short follow-up periods and the evidence from statin trials indicates that the clinical benefits of LDL-lowering may only emerge after about 1 year [51], so these trials may have underestimated the potential impact of longer-term treatment [120,290].

In the FOURIER trial [119], 27564 patients with atherosclerotic CVD, and LDL-C levels of $1.8 \mathrm{mmoL} / \mathrm{L}(70 \mathrm{mg} / \mathrm{dL})$ or higher who were receiving statin therapy, were randomly assigned to receive evolocumab or placebo. Allocation to evolocumab reduced median LDL-C from $2.38 \mathrm{mmoL} / \mathrm{L}(92 \mathrm{mg} / \mathrm{dL})$ at baseline to a mean of $0.78 \mathrm{mmoL} / \mathrm{L}$ $(30 \mathrm{mg} / \mathrm{dL})$ at 48 weeks. After a median follow-up of 2.2 years, evolocumab treatment significantly reduced the risk of the primary endpoint (composite of CV death, MI, stroke, hospitalization for unstable angina, or coronary revascularization) by $15 \%$ [hazard ratio (HR) 0.85 , 95\% CI 0.79-0.92]. An analysis of the time to benefit also showed that there was a lower benefit in the first year than in subsequent years, consistent with the effects of statins observed within the CTT metaanalysis [268]. In the FOURIER trial, allocation to evolocumab did not reduce the risk of CV mortality (HR 1.05, 95\% CI 0.88-1.25) or allcause mortality.

The ODYSSEY Outcomes trial randomized 18924 patients after hospitalization for acute MI or unstable angina, treated with statins, and with LDL-C $\geq 1.8 \mathrm{mmoL} / \mathrm{L}(\geq 70 \mathrm{mg} / \mathrm{dL}$ ), non-HDL cholesterol $\geq 2.6 \mathrm{mmoL} / \mathrm{L}$ ( $\geq 100 \mathrm{mg} / \mathrm{dL}$ ), or ApoB $\geq 80 \mathrm{mg} / \mathrm{dL}$, to receive injections of alirocumab or matching placebo. Allocation to alirocumab reduced the mean baseline LDL-C from $2.38 \mathrm{mmoL} / \mathrm{L}(92 \mathrm{mg} / \mathrm{dL})$ to 1.24 $\mathrm{mmoL} / \mathrm{L}(48 \mathrm{mg} / \mathrm{dL})$ at 12 months. There was a $15 \%$ relative reduction in the primary outcome (composite of CHD death, non-fatal MI, ischaemic stroke, or unstable angina requiring hospitalization) (HR 0.85 , 95\% CI 0.78-0.93) after a median follow-up of 2.8 years [120]. Although there was a significant reduction in all-cause mortality in the ODYSSEY trial, this was an exploratory outcome and was not supported by a significant effect on CV death.

\subsubsection{Adverse effects and interactions}

Anti-PCSK9 mAbs are injected subcutaneously, every other week or once a month, at different doses depending on the agent used. The potential for interaction with orally absorbed drugs is absent, as they will not interfere with pharmacokinetics or pharmacodynamics. Among the most frequently reported side effects are itching at the site of injection and flu-like symptoms [292]. In some studies, an increase of patient-reported neurocognitive effects has been described [293]. However, the EBBINGHAUS trial [231], which was specifically designed to detect neurocognitive function changes, was reassuring, as were the safety reports in both the FOURIER and ODYSSEY trials. Mendelian randomization studies have also suggested that PCSK9 inhibition may increase the risk of DM with an LDL-C-related effect, as apparently occurs for statins [294]. To date, no signal has emerged from RCTs [295-297]. Although large long-term trials of PCSK9 inhibitors are needed to rule out these and other potential side effects of inhibition of PCSK9 [298], 7 year data from the IMPROVE-IT study have shown that prolonged low LDL-C concentrations are not associated with any clear adverse effects [299].

A potential problem of long-term antibody treatment is the occurrence of autoantibodies. Evolocumab and alirocumab are fully human antibodies and, therefore, theoretically less likely to induce autoantibodies. To date, only very few cases of antidrug antibodies have been reported, and no reduction of LDL-C lowering has been observed, but long-term use needs to be monitored. Indeed, the development programme for a third PCSK9 inhibitor, bococizumab, a humanized antibody, was discontinued because of an increase of neutralizing antibodies, which resulted in the attenuation of the LDL-C-lowering effect over time, as well as a higher rate of injection site reactions [300]. However, although PCSK9 inhibitors are very effective drugs that can reduce LDL-C and CV events on top of statin and/or ezetimibe treatment, considering the costs of the treatments and the limited data on long-term safety, these drugs are likely to be considered cost-effective only in those patients at very high-risk of ASCVD, and their use may not be possible in some countries with limited healthcare resources.

\subsection{Lomitapide}

The microsomal TG transfer protein (MTP) transfers TGs and 
phospholipids from the endoplasmic reticulum to ApoB, as a necessary step in the formation of VLDL. MTP inhibition thus prevents the formation of VLDL in the liver and of chylomicrons in the intestine.

Lomitapide is an MTP inhibitor designed for o.d. oral treatment of HoFH. In an open-label, single-arm titration study evaluating lomitapide as adjunct therapy to statins, with or without apheresis and a lowfat diet [301], LDL-C was reduced by $50 \%$ from baseline at 26 weeks and by $44 \%$ at 56 weeks. Lomitapide was also shown to decrease the frequency of apheresis in HoFH patients. It should be noted that the drug's effect on CV outcomes has not yet been determined.

As a consequence of its mechanism of action, lomitapide has been shown to be associated with increased aminotransferase levels, which most likely reflects the increased fat in the liver, as well as poor GI tolerability $[301,302]$. The GI side effects were the most frequent reasons preventing a further increase in the dose of lomitapide in clinical trials [301]. However, it has been noted that the frequency and intensity of GI side effects generally decrease with time. Therefore, prescription of lomitapide requires careful patient education and liver function monitoring during therapy.

\subsection{Mipomersen}

Mipomersen is an antisense oligonucleotide able to bind the messenger RNA (mRNA) of ApoB-100, which triggers the selective degradation of mRNA molecules. After subcutaneous injection, the oligonucleotide is preferentially transported to the liver, where it binds to a specific mRNA preventing the translation of ApoB protein and, consequently, reducing the production of atherogenic lipids and lipoproteins, including LDL and Lp(a) [303]. An adjunct to lipid-lowering medications and diet, mipomersen is indicated to reduce LDL-C in patients with HoFH. Mipomersen is currently approved by the US Food and Drug Administration (FDA), but not by the European Medicines Agency (EMA).

Reactions at the injection site are the most common adverse effects observed in patients treated with mipomersen [304]. However, the main concerns regarding mipomersen's safety are related to liver toxicity. Mipomersen may lead to the development of steatosis. Treated patients have shown a higher increase of liver fat from baseline compared with patients randomized to placebo [303]. The efficacy and safety of long-term mipomersen treatment are currently under evaluation in patients with severe $\mathrm{HeFH}$, and in statin-'intolerant' patients.

\subsection{Fibrates}

\subsubsection{Mechanism of action}

Fibrates are agonists of peroxisome proliferator-activated receptor$\alpha$ (PPAR- $\alpha$ ), acting via transcription factors regulating, among other things, various steps in lipid and lipoprotein metabolism. As a consequence, fibrates have good efficacy in lowering fasting TG levels, as well as post-prandial TGs and TG-rich lipoprotein (TRL) remnant particles.

\subsubsection{Effects on lipids}

Clinical impacts on lipid profiles vary among members of the fibrate class, but are estimated to reach a $50 \%$ reduction of the TG level, a $\leq 20 \%$ reduction of the LDL-C level (but a paradoxical small LDL-C increase may be observed with high TG levels), and an increase of the HDL-C level of $\leq 20 \%$. The magnitude of effect is highly dependent on the baseline lipid levels [305]. Both the HDL-raising and TG-lowering effects of fibrates have been reported to be markedly less $(\sim 5$ and $\sim 20 \%$, respectively) in long-term intervention trials in people with T2DM but without elevated levels of TGs [306,307].

\subsubsection{Effect on cardiovascular morbidity and mortality}

The clinical effects of fibrates have been primarily illustrated by six RCTs: the Helsinki Heart Study (HHS), Veterans Affairs High Density
Lipoprotein Intervention Trial (VA-HIT), Bezafibrate Infarction Prevention (BIP), Lower Extremity Arterial Disease Event Reduction (LEADER), Fenofibrate Intervention and Event Lowering in Diabetes (FIELD), and Action to Control Cardiovascular Risk in Diabetes (ACCORD) trials; in the latter trial, fenofibrate was added to statin therapy [306-311]. In CV outcome trials of fibrates, the risk reduction appeared to be proportional to the degree of non-HDL-C lowering [50].

Although the HHS reported a significant reduction in CVD outcomes with gemfibrozil, neither the FIELD nor the ACCORD studies involving fenofibrate showed a reduction in total CVD outcomes. The LEADER trial included male participants with lower-extremity arterial disease and failed to show that bezafibrate could lead to a clinically important reduction in CVD combined endpoints. Decreases in the rates of nonfatal MI were reported, although often as a result of post hoc analyses. The effect was most evident in people with elevated TG/low HDL-C levels. However, the data on other outcome parameters have remained equivocal. Only one study, ACCORD, has analysed the effect of a fibrate as an add-on treatment to a statin. No overall benefit was reported in two recent meta-analyses [312,313]. Results from other meta-analyses suggest reduced major CVD events in patients with high TGs and low HDL-C in fibrate-treated patients, but no decrease in CVD or total mortality [314-316]. Thus, the overall efficacy of fibrates on CVD outcomes is much less robust than that of statins. Recently, a new selective PPAR- $\alpha$ modulator (pemafibrate) has been reported to have marked efficacy in reducing TRLs [317]. The study, Pemafibrate to Reduce Cardiovascular OutcoMes by Reducing Triglycerides IN PatiENts With DiabeTes (PROMINENT), is an ongoing CVD outcome trial designed to evaluate the efficacy of pemafibrate in some 10000 highrisk diabetic patients with high TG and low HDL-C levels [318]. Overall, the potential CV benefits of fibrates require further confirmation.

\subsubsection{Adverse effects and interactions}

Fibrates are generally well tolerated with mild adverse effects, GI disturbances being reported in $<5 \%$ of patients, and skin rashes in $2 \%$ [319]. In general, myopathy, liver enzyme elevations, and cholelithiasis represent the most well-known adverse effects associated with fibrate therapy [319]. The risk of myopathy has been reported to be 5.5-fold greater with fibrate monotherapy (mainly with gemfibrozil) compared with a statin, and it varies with different fibrates and statins used in combination. This is explained by the pharmacological interactions between the metabolism of different fibrates and pathways of glucuronidation of statins. Gemfibrozil inhibits the metabolism of statins via the glucuronidation pathway, which leads to marked increases in plasma concentrations of statins [320]. As fenofibrate does not share the same pharmacokinetic pathways as gemfibrozil, the risk of myopathy is much less with this combination therapy [319].

As a class, fibrates have been reported to raise both serum creatinine and homocysteine levels in both short- and long-term studies. The increase of serum creatinine by fibrate therapy seems to be fully reversible when the drug is stopped. Data from meta-analyses suggest that a reduction of calculated glomerular filtration rate (GFR) does not reflect any adverse effects on kidney function [315]. Fibrates are associated with a slightly increased risk of pancreatitis [321]. The increase in homocysteine levels caused by fibrates has been considered to be relatively neutral with respect to CVD risk. However, a fibrate-induced increase in homocysteine may blunt elevation of both HDL-C and ApoA1 levels, and this effect may contribute to the smaller than estimated benefits of fenofibrate in CV outcome parameters [322].

\section{8. n-3 fatty acids}

\subsubsection{Mechanism of action}

The n-3 (or omega-3) fatty acids [eicosapentaenoic acid (EPA) and docosahexaenoic acid (DHA)] can be used at pharmacological doses to lower TGs. n-3 fatty acids (2-4 g/day) affect serum lipids and 
lipoproteins, in particular VLDL concentrations. The underlying mechanism is poorly understood, although it may be related, at least in part, to their ability to interact with PPARs and to decreased secretion of ApoB.

\subsubsection{Effects on lipids}

n-3 fatty acids reduce TGs, but their effects on other lipoproteins are trivial. More detailed data on clinical outcomes are needed to justify wide use of prescription n-3 fatty acids [323]. The recommended doses of total EPA and DHA to lower TGs have varied between 2 and $4 \mathrm{~g}$ /day. Three recent studies in people with high TGs using EPA reported a significant reduction in serum TG levels of up to $45 \%$ in a dose-dependent manner [324-326]. The efficacy of omega-3 fatty acids in lowering serum TGs has also been reported in meta-analyses [157]. Recently, the EpanoVa fOr Lowering Very high triglyceridEs II (EVOLVE II) trial confirmed the efficacy of omega-3 fatty acids in lowering serum TGs [327].

\subsubsection{Effect on cardiovascular morbidity and mortality}

A Cochrane meta-analysis, including 112059 people from 79 trials, reported no overall effect of omega-3 PUFAs on total mortality (relative risk $0.98,95 \% \mathrm{CI} 0.90-1.03$ ) or CV events (relative risk $0.99,95 \% \mathrm{Cl}$ 0.94-1.04), with only a suggestion that omega-3 fatty acids reduced CHD events (relative risk $0.93,95 \% \mathrm{Cl} 0.88-0.97$ ) [328]. Recently, the A Study of Cardiovascular Events iN Diabetes (ASCEND) trial [329], which randomly assigned 15480 patients with DM but without atherosclerotic CV disease to n-3 fatty acids or placebo, showed no significant difference in the risk of serious vascular events after a mean follow-up of 7.4 years (relative risk 1.00, 95\% Cl 0.91-1.09).

The data remain inconclusive and the clinical efficacy of omega- 3 fatty acids appears to be related to non-lipid effects [330,331]. Moreover, studies with omega-3 fatty acids have suffered from the dose used ( $1 \mathrm{~g} /$ day), which does not affect plasma lipids to a large extent, as the dose required to decrease plasma TGs is $>2 \mathrm{~g} /$ day. The Reduction of Cardiovascular Events with EPA-Intervention Trial (REDUCE-IT) [195] aimed to evaluate the potential benefits of omega-3 oil (EPA) on ASCVD outcomes in people with elevated serum TGs; the trial enrolled $\sim 8000$ patients on statin therapy, with LDL-C levels between 1.0 and 2.6 $\mathrm{mmoL} / \mathrm{L}(41-100 \mathrm{mg} / \mathrm{dL})$ and various $\mathrm{CV}$ risk factors, including persistent elevated TGs between 1.7 and $5.6 \mathrm{mmoL} / \mathrm{L}(150-499 \mathrm{mg} / \mathrm{dL})$, and either established ASCVD or DM, and at least one other CV risk factor. Use of high doses ( $2 \mathrm{~g}$ b.i.d.) of EPA as compared with placebo (mineral oil) resulted in a $\sim 25 \%$ relative risk reduction $(P<0.001)$ in major adverse CV events (MACE). Another randomized placebo-controlled trial, Outcomes Study to Assess STatin Residual Risk Reduction with EpaNova in HiGh CV Risk PatienTs with Hypertriglyceridemia (STRENGTH) [332], which aims to determine whether reduction of TRLs and remnants in statin-treated patients will provide additional ASCVD risk reduction, is ongoing. The VITamin D and OmegA-3 TriaL (VITAL), which reported recently, was a $2 \times 2$ factorial design study in which healthy participants were randomized in a 1:1 fashion to either vitamin D3 (at a dose of 2000 IU per day) vs. matching placebo, and n-3 fatty acids ( $1 \mathrm{~g}$ per day as a fish-oil capsule containing $840 \mathrm{mg}$ of $\mathrm{n}-3$ fatty acids, including $460 \mathrm{mg}$ of EPA and $380 \mathrm{mg}$ of DHA) vs. matching placebo. It showed that supplementation with either $\mathrm{n}-3$ fatty acids at a dose of $1 \mathrm{~g} /$ day, or vitamin D3 at a dose of $2000 \mathrm{IU} /$ day, was not effective for primary prevention of $\mathrm{CV}$ or cancer events among healthy middle-aged men and women over 5 years of follow-up [333].

\subsubsection{Safety and interactions}

The administration of $n-3$ fatty acids appears to be safe and devoid of clinically significant interactions. The most common side effect is GI disturbance. The antithrombotic effects may increase the propensity for bleeding, especially when given in addition to aspirin/clopidogrel. Recently, data from one study have associated a risk of prostate cancer with high dietary intake of n-3 PUFAs [334].

\subsection{Nicotinic acid}

Nicotinic acid has key action sites in both the liver and adipose tissue. In the liver, nicotinic acid inhibits diacylglycerol acyltransferase2 resulting in decreased secretion of VLDL particles, which is also reflected in reductions of plasma levels of both IDL and LDL particles [335]. Nicotinic acid primarily raises HDL-C and ApoA1 by stimulating ApoA1 production in the liver [335]. Two large randomized trials with nicotinic acid—one with extended-release niacin [66] and one with niacin plus laropiprant [67] - have shown no beneficial effect and an increased frequency of serious adverse effects. No medication containing nicotinic acid is currently approved in Europe.

\subsection{Cholesteryl ester transfer protein inhibitors}

To date, the pharmacological approach that has led to the greatest elevations in HDL-C levels has been direct inhibition of CETP by smallmolecule inhibitors, which may induce an increase in HDL-C by $\geq 100 \%$ on a dose-dependent basis. Torcetrapib was studied in the Investigation of Lipid Level Management to Understand its Impact in Atherosclerotic Events (ILLUMINATE) trial, which was stopped early due to increased mortality [336]. Dalcetrapib raises HDL-C levels by $30-40 \%$ with no appreciable effect on LDL-C, offering specific insight into pure HDL-C raising. However, dalcetrapib failed to show any benefit in ACS patients in the dal-OUTCOMES trial. Evacetrapib, which raises HDL-C levels by $130 \%$ and lowers LDL-C by $37 \%$, was studied in the ACCELERATE trial [63], which was terminated due to futility. Recently, anacetrapib, which raises HDL-C and ApoA-I levels (by 104 and $36 \%$, respectively), and lowers LDL-C and ApoB (by 17 and 18\%, respectively), was studied in the REVEAL trial. Anacetrapib reduced major coronary events by $9 \%$ over a median of 4.1 years [64]. The magnitude of the relative risk reduction appeared to be consistent with the magnitude of LDL-C- or non-HDL-C-level lowering [337]. This drug has not been submitted for regulatory approval.

\subsection{Future perspectives}

\subsubsection{New approaches to reduce low-density lipoprotein cholesterol}

An alternative approach targeting PCSK9 consists of RNA interference. In a phase I and a phase II trial, the small interfering RNA (siRNA) molecule inclisiran-which inhibits the synthesis of PCSK9-reduced LDL-C by up to 50\% and the reduction was dose-dependent. Reductions in PCSK9 and LDL-C levels were maintained for $\leq 6$ months $[338,339]$. No specific serious adverse events were observed. HPS4/TIMI65/ORION4, with a planned mean duration of 5 years, is currently comparing inclisiran vs. placebo among 15000 patients with a prior MI or stroke.

Bempedoic acid is a novel, first-in-class, oral small molecule that inhibits cholesterol synthesis by inhibiting the action of ATP citrate lyase, a cytosolic enzyme upstream of 3-hydroxy-3-methylglutarylcoenzyme A reductase [340]. So far, it has been tested in diabetic patients, and patients with or without statin 'intolerance'. In monotherapy, bempedoic acid reduces LDL-C levels by $\sim 30 \%$ and by about $50 \%$ in combination with ezetimibe. Bempedoic acid is currently being tested in phase III trials and some trials have been completed [341,342]. 
8.11.2. New approaches to reduce triglyceride-rich lipoproteins and their remnants

As genetic studies indicate that angiopoietin-like protein 3 (ANGPTL3) deficiency protects against atherosclerotic disease and that this relationship is causal [343], an ANGPTL3 antibody (evinacumab) is being developed. Evinacumab has been shown to decrease TGs, LDL-C, and Lp(a) levels in HoFH patients [344]. Another approach that is currently being investigated is the inhibition of ANGPTL3 production by antisense oligonucleotides [345]. IONIS-ANGPTL3-LRx, an antisense oligonucleotide targeting ANGPTL3, another critical protein in the clearance of TRLs, reduces plasma TGs by about $85 \%$. Thus, the future may yield tools to improve TRL clearance that will be reflected in the atherogenic load of the remnant particles.

The rapid development of gene-silencing technology has allowed proteins (ApoC-III) that are critical in the regulation of TRL clearance processes to be targeted. A second-generation antisense oligonucleotide targeting ApoC-III mRNA has been developed [346]. Two phase III trials have evaluated the safety and efficacy of volanesorsen in patients with elevated TG levels [347,348]. Volanesorsen reduced plasma TGs by $\sim 70 \%$ and ApoC-III by $80-90 \%$ [349]. The EMA recently issued a marketing authorization for Waylivra (volanesorsen) as an adjunct to diet in adult patients with genetically confirmed familial chylomicronaemia syndrome (FCS) who are at high-risk for pancreatitis, in whom response to diet and TG-lowering therapy has been inadequate.

\subsubsection{New approaches to increase high-density lipoprotein cholesterol}

Although genetic studies suggest that low HDL-C levels are not a cause of ASCVD, casting doubt on the possibilities of future treatment options to raising HDL-C levels with attenuation of CVD, major developments in the search for efficacious agents to raise HDL-C and ApoA1 levels with concomitant benefits on atherosclerosis and CV events are on the horizon. On the one hand, interest is focused on ApoA1 mimetic peptides and recombinant forms of HDL possessing potential for in vivo HDL particle remodelling and enhanced cardioprotective activity [350]. On the other, agents that enhance catabolism of TG-rich lipoproteins, such as the antisense oligonucleotide to ApoC-III, and which lead to a concomitant reduction in TGs $(\sim 70 \%)$ and a marked elevation in HDL-C $(\sim 40 \%)$ in hypertriglyceridemia, are under development [351]. Importantly, however, we currently lack understanding of the relationship between the modality of raising HDL/ApoA-I levels and a possible antiatherogenic function of HDL particles.

\subsubsection{New approaches to reduce lipoprotein(a) levels}

Another approach under study is the selective decrease of Lp(a) concentrations. RNA-based therapies are now being evaluated in clinical settings. Results from studies of an antisense oligonucleotide in patients with normal $\mathrm{Lp}$ (a) values as well as in patients with elevated Lp (a) concentrations have shown a reduction of $>90 \%$ [352]. These approaches are currently being evaluated in phase II-III studies and an outcome trial is planned to study whether $\operatorname{Lp}(\mathrm{a})$ reduction translates into risk reduction.

\subsection{Strategies to control plasma cholesterol}

Although LDL-C goals are attained with monotherapy in many patients, a significant proportion of patients at high-risk or with very high LDL-C levels need additional treatment. In this case, combination therapy is reasonable. In patients at very-high risk and with persistent high-risk despite being treated with a maximally tolerated statin, combination with ezetimibe is recommended and, if still not at goal, the addition of a PCSK9 inhibitor is recommended (see Fig. 4 and Recommendations for pharmacological low-density lipoprotein cholesterol lowering). Of note, addition of a PCSK9 inhibitor directly to a statin is also feasible [120,290] (Fig. 4).

Recommendations for pharmacological low-density lipoprotein cholesterol lowering.

\begin{tabular}{|c|c|c|}
\hline Recommendations & Class $^{\mathrm{a}}$ & Level $^{\mathbf{b}}$ \\
\hline $\begin{array}{l}\text { It is recommended that a high-intensity statin is prescribed up to the highest tolerated dose to reach the goals set for the } \\
\text { specific level of risk. }\end{array}$ & $\mathbf{I}$ & A \\
\hline $\begin{array}{l}\text { If the goals }{ }^{c} \text { are not achieved with the maximum tolerated dose of a statin, combination with ezetimibe is } \\
\text { recommended. } .^{33}\end{array}$ & $\mathbf{I}$ & B \\
\hline $\begin{array}{l}\text { For primary prevention patients at very-high risk, but without FH, if the LDL-C goal is not achieved on a maximum toler- } \\
\text { ated dose of a statin and ezetimibe, a combination with a PCSK9 inhibitor may be considered. }\end{array}$ & Illb & C \\
\hline $\begin{array}{l}\text { For secondary prevention, patients at very-high risk not achieving their goal }{ }^{\mathrm{c}} \text { on a maximum tolerated dose of a statin and } \\
\text { ezetimibe, a combination with a PCSK9 inhibitor is recommended. }{ }^{19,120}\end{array}$ & $\mathbf{I}$ & A \\
\hline $\begin{array}{l}\text { For very-high-risk FH patients (that is, with ASCVD or with another major risk factor) who do not achieve their goal }{ }^{c} \text { on } \\
\text { a maximum tolerated dose of a statin and ezetimibe, a combination with a PCSK9 inhibitor is recommended. }\end{array}$ & $\mathbf{I}$ & C \\
\hline If a statin-based regimen is not tolerated at any dosage (even after rechallenge), ezetimibe should be considered. ${ }^{197,265,353}$ & Ila & C \\
\hline $\begin{array}{l}\text { If a statin-based regimen is not tolerated at any dosage (even after rechallenge), a PCSK9 inhibitor added to ezetimibe } \\
\text { may also be considered. }\end{array}$ & Ilb & C \\
\hline If the goal ${ }^{c}$ is not achieved, statin combination with a bile acid sequestrant may be considered. & Illb & C \\
\hline
\end{tabular}

ASCVD = atherosclerotic cardiovascular disease; FH = familial hypercholesterolaemia; LDL-C = low-density lipoprotein cholesterol; PCSK9 = proprotein convertase subtilisin/kexin type 9.

${ }^{a}$ Class of recommendation.

${ }^{\mathrm{b}}$ Level of evidence.

${ }^{\mathrm{c}}$ For definitions see Table 7. 


\section{Intensity of lipid lowering treatment}

\section{Treatment}

Moderate intensity statin

High intensity statin

High intensity statin plus

ezetimibe

PCSK9 inhibitor

PCSK9 inhibitor plus high intensity statin

PCSK9 inhibitor plus high intensity statin plus ezetimibe
Average LDL-C reduction

$$
\begin{aligned}
& \approx 30 \% \\
& \approx 50 \% \\
& \approx 65 \% \\
& \approx 60 \% \\
& \approx 75 \% \\
& \approx 85 \%
\end{aligned}
$$


A

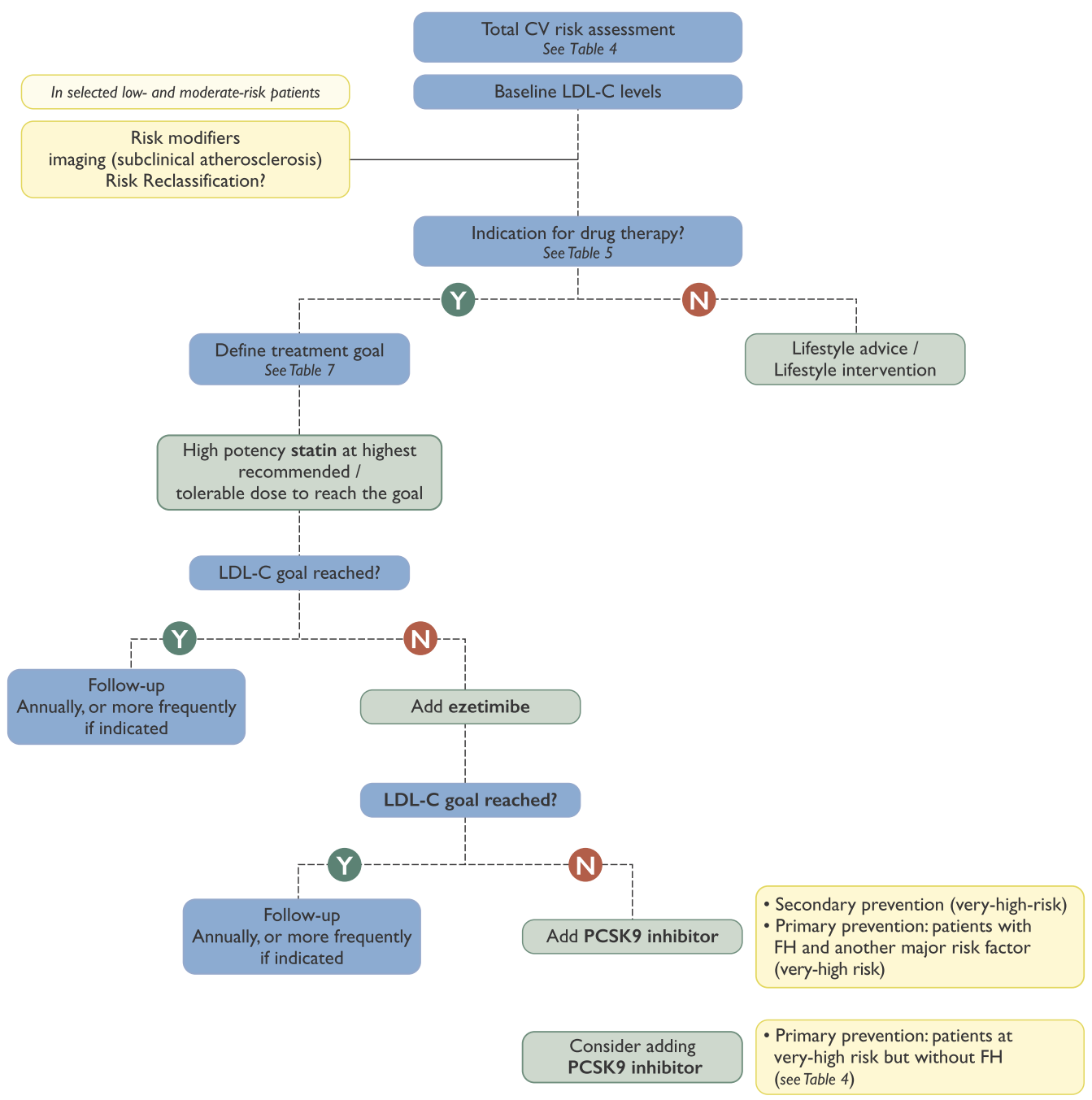

B Treatment goal for LDL-C

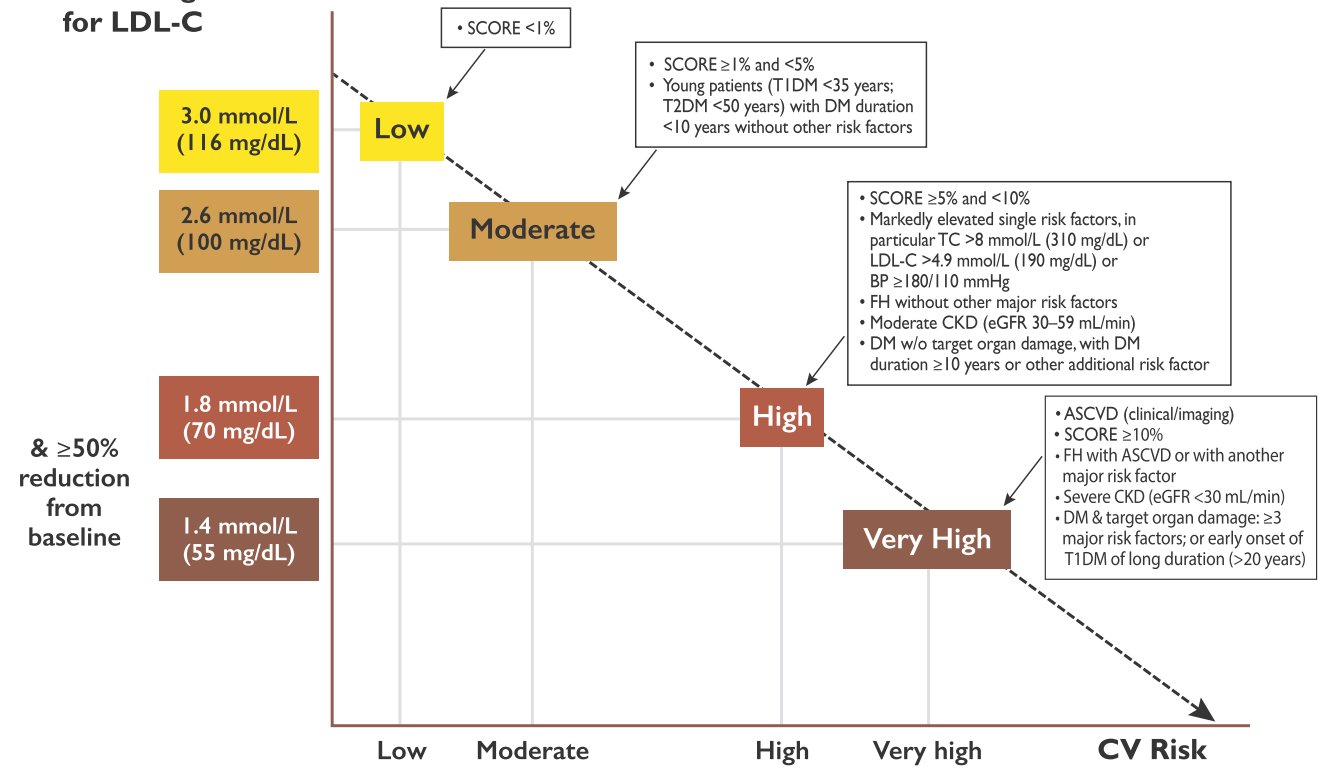

Fig. 4. Treatment goals and algorithm for low-density lipoprotein cholesterol lowering according to cardiovascular disease risk. $(A)$ Treatment goals for low-density lipoprotein cholesterol across categories of total cardiovascular disease risk. (B) Treatment algorithm for pharmacological low-density lipoprotein cholesterol lowering. ASCVD = atherosclerotic cardiovascular disease; $\mathrm{BP}=$ blood pressure; $\mathrm{CKD}=$ chronic kidney disease; $\mathrm{CV}=$ cardiovascular; $\mathrm{DM}=$ diabetes mellitus; eGFR $=$ estimated glomerular filtration rate; FH $=$ familial hypercholesterolaemia; LDL-C $=$ low-density lipoprotein cholesterol; PCSK9 $=$ proprotein convertase subtilisin/kexin type 9; SCORE $=$ Systematic Coronary Risk Estimation; T1DM = type $1 \mathrm{DM}$; T2DM = type $2 \mathrm{DM}$; TC $=$ total cholesterol. 
Recommendations for drug treatment of patients with hypertriglyceridaemia.

\begin{tabular}{|c|c|c|}
\hline Recommendations & Class $^{\mathrm{a}}$ & Level $^{\mathrm{t}}$ \\
\hline $\begin{array}{l}\text { Statin treatment is recommended as the first } \\
\text { drug of choice to reduce CVD risk in high-risk } \\
\text { individuals with hypertriglyceridaemia [TG lev- } \\
\text { els }>2.3 \mathrm{mmol} / \mathrm{L}(>200 \mathrm{mg} / \mathrm{dL})]^{355}\end{array}$ & I & B \\
\hline $\begin{array}{l}\text { In high-risk (or above) patients with TG levels } \\
\text { between } 1.5-5.6 \mathrm{mmol} / \mathrm{L}(135-499 \mathrm{mg} / \mathrm{dL} \text { ) } \\
\text { despite statin treatment, } \mathrm{n}-3 \text { PUFAs (icosapent } \\
\text { ethyl } 2 \times 2 \mathrm{~g} / \text { day) should be considered in } \\
\text { combination with a statin. }^{194}\end{array}$ & Ila & B \\
\hline $\begin{array}{l}\text { In primary prevention patients who are at } \\
\text { LDL-C goal with TG levels }>2.3 \mathrm{mmol} / \mathrm{L} \\
\text { (>200 } \mathrm{mg} / \mathrm{dL} \text { ), fenofibrate or bezafibrate may } \\
\text { be considered in combination with } \\
\text { statins. }^{305-307,356}\end{array}$ & Ilb & B \\
\hline $\begin{array}{l}\text { In high-risk patients who are at LDL-C goal } \\
\text { with TG levels }>2.3 \mathrm{mmol} / \mathrm{L}(>200 \mathrm{mg} / \mathrm{dL}) \text {, } \\
\text { fenofibrate or bezafibrate may be considered } \\
\text { in combination with statins. }{ }^{305-307,356}\end{array}$ & Ilb & C \\
\hline
\end{tabular}

CVD = cardiovascular disease; LDL-C = low-density lipoprotein cholesterol; PUFA = polyunsaturated fatty acids; $\mathrm{TG}=$ triglyceride.

a Class of recommendation.

${ }^{\mathrm{b}}$ Level of evidence.

\section{Management of dyslipidaemias in different clinical settings}

\subsection{Familial dyslipidaemias}

Plasma lipid levels are, to a very large extent, determined by genetic factors. In its more extreme forms this is manifested as familial dyslipidaemias. A number of monogenic lipid disorders have been identified; among these, $\mathrm{FH}$ is the most common and is strongly related to CVD (Table 11). In general, in a patient with dyslipidaemia, the pattern of inheritance commonly does not suggest that there is a major single gene (monogenic) disorder causing the abnormality; rather, it stems from the inheritance of more than one gene variant affecting lipoprotein metabolism that, on its own, might have relatively little effect, but in combination with another or others has a greater influence on TC, TGs, or HDL-C. The pattern of inheritance is polygenic [357]. It is common to find that high LDL-C, high TG, or low HDL-C levels affect several family members.

\subsubsection{Familial combined hyperlipidaemia}

Familial combined hyperlipidaemia $(\mathrm{FCH})$ is a highly prevalent mixed dyslipidaemia (1:100-200) characterized by elevated levels of LDL-C, TGs, or both, and is an important cause of premature CAD. FCH is a complex disease, and the phenotype is determined by the interaction of multiple susceptibility genes and the environment. It has considerable overlap with the dyslipidaemic phenotypes of T2DM and MetS. Even within a family, the phenotype shows high inter- and intrapersonal variability based on lipid values (TGs, LDL-C, HDL-C, and ApoB). FCH has no monogenic component and is not linked to a single genetic cause, but the phenotype is high LDL-C and/or high TGs [358,359]. Therefore, the diagnosis is commonly missed in clinical practice; the combination of ApoB $>120 \mathrm{mg} / \mathrm{dL}$ and TGs $>1.5 \mathrm{mmoL} /$ $\mathrm{L}$ ( $>133 \mathrm{mg} / \mathrm{dL}$ ) with a family history of premature CVD can be used to identify people who most probably have FCH [360].

The concept of mixed dyslipidaemia is also valuable clinically in assessing CV risk. It emphasizes both the importance of considering family history in deciding how rigorously to treat dyslipidaemia and that elevated LDL-C levels portend a higher risk when HTG is also present. Statin treatment decreases $\mathrm{CV}$ risk by the same relative amount in people with HTG as in those without. Because the absolute risk is often greater in those with HTG, they may therefore benefit greatly from LDL-lowering therapy.

\subsubsection{Familial hypercholesterolaemia}

9.1.2.1. Heterozygous familial hypercholesterolaemia. FH is a common codominant monogenic dyslipidaemia causing premature CVD due to lifelong elevation of plasma levels of LDL-C. If left untreated, men and women with $\mathrm{HeFH}$ typically develop early CAD before the ages of 55 and 60 years respectively. The risk of CHD among individuals with definite or probable $\mathrm{HeFH}$ is estimated to be increased at least 10-fold. However, early diagnosis and appropriate treatment can dramatically reduce the risk for $\mathrm{CAD}$.

The prevalence of $\mathrm{HeFH}$ in the population is estimated to be $1 /$ $200-250,{ }^{361}$ translating to a total number of cases between 14 and 34 million worldwide [362,363]. Only a minor fraction of these cases is identified and properly treated.

FH is a monogenic disease caused by loss-of-function mutations in the $L D L R$ or $a p o B$ genes, or a gain-of-function mutation in the PCSK9 gene; around $95 \%$ of $\mathrm{FH}$ cases are caused by mutations in $L D L R$. More than 1000 different mutations that cause $\mathrm{FH}$ have been identified in $L D L R$. The different mutations cause reduced function or complete lossof-function, the latter being associated with more severe hypercholesterolaemia and CVD.

The diagnosis of FH is usually based on clinical presentation. The commonly used criteria from the Dutch Lipid Clinic Network are shown in Table 12. Other criteria are the Simon Broome register or the WHO criteria [364,365].

The diagnosis can be verified by showing causative mutations in the pathogenic genes. However, in most studies, the frequency of detectable mutations in patients with a clinically definite or probable HeFH is between 60 and $80 \%$. This suggests that a considerable proportion of patients with $\mathrm{FH}$ have either a polygenic cause of the disease or that other genes, yet to be identified, are involved.

Genetic testing and cascade screening. Probands (index cases) should be identified according to the following criteria:

- $\mathrm{TC} \geq 8 \mathrm{mmoL} / \mathrm{L}$ ( $\geq 310 \mathrm{mg} / \mathrm{dL})$ without treatment in an adult or adult family member (or $>95$ th percentile by age and gender for country);

- Premature CHD in the patient or a family member;

- Tendon xanthomas in the patient or a family member; or

- Sudden premature cardiac death in a family member.

Cascade screening of family members of a known index case allows for the efficient identification of new cases. Cascade screening is best performed by a lipid clinic. In most families, the cases may be identified with TC or LDL-C analysis; however, genetic testing is recommended when the causative mutation is known.

Cholesterol-lowering treatment should be initiated as soon as possible after a diagnosis has been made. To improve risk assessment, the use of imaging techniques to detect asymptomatic atherosclerosis is recommended. The concept of cumulative cholesterol burden illustrates the importance of early treatment (for children, see below). Treatment should be initiated with high-intensity statin therapy, in most cases in combination with ezetimibe. In FH patients at very-high risk of ASCVD due to a prior history of ASCVD or another major risk factor, LDL-C goals are a $\geq 50 \%$ reduction of LDL-C from baseline and an LDL-C $<1.4 \mathrm{mmoL} / \mathrm{L}$ ( $<55 \mathrm{mg}$ / $\mathrm{dL}$ ). In the absence of ASCVD or another major risk factor, patients with FH are categorized as high-risk, and LDL-C goals are a $\geq 50 \%$ reduction of LDL-C from baseline and an LDL-C $<1.8 \mathrm{mmoL} / \mathrm{L}(<70 \mathrm{mg} / \mathrm{dL})$.

PCSK9 inhibitors lower LDL-C levels by up to $60 \%$ on top of statins. Two RCTs have reported a beneficial effect on clinical endpoints in ASCVD patients without FH $[119,120]$. PCSK9 inhibitors are recommended in very-high-risk patients with $\mathrm{FH}$ if the treatment goal is 
not achieved on maximal tolerated statin plus ezetimibe. PCSK9 inhibitors are also recommended in FH patients who cannot tolerate statins [366,367].

Recommendations for the detection and treatment of patients with HeFH are shown below.

Recommendations for the detection and treatment of patients with heterozygous familial hypercholesterolaemia.

\begin{tabular}{|c|c|c|}
\hline Recommendations & Class $^{a}$ & Level $^{\mathrm{t}}$ \\
\hline $\begin{array}{l}\text { It is recommended that a diagnosis of } \mathrm{FH} \text { is } \\
\text { considered in patients with } \mathrm{CHD} \text { aged }<55 \\
\text { years for men and }<60 \text { years for women, in } \\
\text { people with relatives with premature fatal or } \\
\text { non-fatal CVD, in people with relatives who } \\
\text { have tendon xanthomas, in people with } \\
\text { severely elevated LDL-C [in adults }>5 \mathrm{mmol} / \mathrm{L} \\
(>190 \mathrm{mg} / \mathrm{dL}) \text {, in children }>4 \text { mmol/L }(>150 \\
\mathrm{mg} / \mathrm{dL})] \text {, and in first-degree relatives of } \mathrm{FH} \\
\text { patients. }\end{array}$ & I & C \\
\hline $\begin{array}{l}\text { It is recommended that FH should be diag- } \\
\text { nosed using clinical criteria and confirmed, } \\
\text { when possible, via DNA analysis. }\end{array}$ & I & C \\
\hline $\begin{array}{l}\text { Once the index case is diagnosed, family cas- } \\
\text { cade screening is recommended. }\end{array}$ & I & C \\
\hline $\begin{array}{l}\text { It is recommended that FH patients with } \\
\text { ASCVD or who have another major risk fac- } \\
\text { tor are treated as very-high-risk, and that } \\
\text { those with no prior ASCVD or other risk fac- } \\
\text { tors are treated as high-risk. }\end{array}$ & I & C \\
\hline $\begin{array}{l}\text { For FH patients with ASCVD who are at very- } \\
\text { high risk, treatment to achieve a } \geq 50 \% \text { reduc- } \\
\text { tion from baseline and an LDL-C }<1.4 \mathrm{mmol} / \mathrm{L} \\
\text { ( }<55 \mathrm{mg} / \mathrm{dL} \text { ) is recommended. If goals cannot } \\
\text { be achieved, a drug combination is } \\
\text { recommended. }\end{array}$ & I & C \\
\hline $\begin{array}{l}\text { In primary prevention, for individuals with } \mathrm{FH} \\
\text { at very-high risk, an LDL-C reduction of } \geq 50 \% \\
\text { from baseline and an LDL-C goal of }<1.4 \\
\mathrm{mmol} / \mathrm{L}(<55 \mathrm{mg} / \mathrm{dL}) \text { should be considered. }\end{array}$ & Ila & C \\
\hline $\begin{array}{l}\text { Treatment with a PCSK9 inhibitor is recom- } \\
\text { mended in very-high-risk FH patients if the } \\
\text { treatment goal is not achieved on maximal tol- } \\
\text { erated statin plus ezetimibe. }\end{array}$ & I & C \\
\hline $\begin{array}{l}\text { In children, testing for } \mathrm{FH} \text { is recommended } \\
\text { from the age of } 5 \text { years, or earlier if } \mathrm{HoFH} \text { is } \\
\text { suspected. }\end{array}$ & I & C \\
\hline $\begin{array}{l}\text { Children with } \mathrm{FH} \text { should be educated to adopt } \\
\text { a proper diet and treated with a statin from } \\
8-10 \text { years of age. Goals for treatment should } \\
\text { be LDL-C }<3.5 \mathrm{mmol} / \mathrm{L}(<135 \mathrm{mg} / \mathrm{dL}) \text { at }>10 \\
\text { years of age. }\end{array}$ & Ila & C \\
\hline
\end{tabular}

ASCVD = atherosclerotic cardiovascular disease; CHD = coronary heart disease; $\mathrm{CVD}=$ cardiovascular disease; $\mathrm{FH}=$ familial hypercholesterolaemia; HoFH $=$ homozygous $\quad \mathrm{FH} ; \quad$ LDL-C $=$ low-density lipoprotein cholesterol; PCSK9 = proprotein convertase subtilisin/kexin type 9 .

${ }^{\text {a }}$ Class of recommendation.

${ }^{\mathrm{b}}$ Level of evidence.
9.1.2.2. Homozygous familial hypercholesterolaemia. HoFH is a rare and life-threatening disease. The clinical picture is characterized by extensive xanthomas, marked premature and progressive CVD, and TC $>13 \mathrm{mmoL} / \mathrm{L}(>500 \mathrm{mg} / \mathrm{dL})$. Most patients develop CAD and aortic stenosis before the age of 20 years and die before 30 years of age. The frequency of HoFH is estimated to be $1 / 160000-1 / 320000$. The early identification of these children and prompt referral to a specialized clinic is crucial. The patients should be treated with intensive LDL-lowering drug therapy and, when available, with lipoprotein apheresis. This treatment (every 1-2 weeks) can decrease plasma LDL-C levels by $55-70 \%$. The procedure frequency may be adjusted for each patient as lipid levels, symptoms, and other diseaserelated parameters change. Maximally tolerated pharmacological therapy must be maintained [368]. For a more detailed discussion on HoFH, see the EAS consensus statements [366,368].

9.1.2.3. Familial hypercholesterolaemia in children. $\mathrm{FH}$ is diagnosed in children based on phenotypic criteria including elevated LDL-C plus a family history of elevated LDL-C, premature CAD, and/or positive genetic testing [369]. Testing during childhood is optimal to discriminate between FH and non-FH using LDL-C. In children with a family history of high cholesterol or premature CHD, an accepted cutoff is $\geq 4.0 \mathrm{mmoL} / \mathrm{L}$ ( $\geq 160 \mathrm{mg} / \mathrm{dL})$. If a parent has a known genetic defect, the diagnostic level for the child is $\geq 3.5 \mathrm{mmoL} / \mathrm{L}$ ( $\geq 130 \mathrm{mg}$ / $\mathrm{dL}$ ). If possible, genetic testing of the child is suggested.

Although there have been no placebo-controlled trials in children, observational studies have suggested that early treatment can reduce LDL-C burden, improve endothelial function, substantially attenuate the development of atherosclerosis, and improve coronary outcomes [369-371]. Treatment of children with FH includes a healthy lifestyle and statin treatment. A heart-healthy diet should be adopted early in life and statin treatment should be considered at 6-10 years of age. Statin treatment should be started with low doses and the dose should be increased to reach goals [372]. The goal in children $>10$ years of age is an LDL-C $<3.5 \mathrm{mmoL} / \mathrm{L}(<135 \mathrm{mg} / \mathrm{dL})$ and at younger ages a $\geq 50 \%$ reduction of LDL-C.

\subsubsection{Familial dysbetalipoproteinaemia}

Familial dysbetalipoproteinaemia (i.e. type III hyperlipoproteinaemia; remnant removal disease) is rare and is generally inherited as an autosomal recessive disorder with variable penetrance. Familial dysbetalipoproteinaemia produces a characteristic clinical syndrome in which both TC and TGs are elevated before treatment, usually both in the range of 7-10 mmoL/L. In severe cases, patients develop tuberoeruptive xanthomas, particularly over the elbows and knees, and palmar xanthomata in the skin creases of the hands and wrists. The risk of CAD is very high, and accelerated atherosclerosis of the femoral and tibial arteries is also prevalent. The syndrome is usually not expressed at a young age or in women before menopause. The majority of cases are homozygous for the E2 isoform of ApoE. ApoE is important for the hepatic clearance of chylomicron remnants and IDL. ApoE2 binds less readily than the E3 and E4 isoforms to hepatic receptors. However, without some coincidental cause of dyslipidaemia such as dyslipidaemia associated with HTG, DM, obesity, or hypothyroidism [373-375], ApoE2 homozygosity does not generally cause familial dysbetalipoproteinaemia.

The detection of ApoE2 homozygosity in a dyslipidaemic patient is diagnostic and analysis of ApoE isoforms is now available in most clinical laboratories. The presence of cholesterol remnants characteristic of familial dysbetalipoproteinaemia can be reliably predicted on the basis of plasma levels of cholesterol, TGs, and ApoB [376]. If suspicion is confirmed, ApoE genotyping can be performed. In older patients with xanthomata resembling those of familial dysbetalipoproteinaemia who prove not to be homozygous for ApoE2, a paraprotein should be sought. The treatment of familial dysbetalipoproteinaemia should be undertaken in a specialist clinic. Most cases respond well to 
Table 11

Genetic disorders of lipoprotein metabolism.

\begin{tabular}{|c|c|c|c|}
\hline Disorder & Prevalence & Gene(s) & Effect on lipoproteins \\
\hline $\mathrm{HeFH}$ & 1 in $200-250$ & $\begin{array}{l}\text { LDLR } \\
\text { APO B } \\
\text { PCSK9 }\end{array}$ & $\uparrow L D L-C$ \\
\hline HoFH & 1 in $160000-320000$ & $\begin{array}{l}\text { LDLR } \\
\text { APO B } \\
\text { PCSK9 }\end{array}$ & $\uparrow \uparrow L D L-C$ \\
\hline $\mathrm{FCH}$ & 1 in $100 / 200$ & USF1 + modifying genes & $\uparrow L D L-C \uparrow$ VLDL-C $\uparrow A p o B$ \\
\hline Familial dysbetalipoproteinaemia & 1 in 5000 & $A P O E$ & $\uparrow \uparrow$ IDL and chylomicron remnants ( $\beta \mathrm{VLDL})$ \\
\hline Familial lipoprotein lipase deficiency (familial chylomicron syndrome) & 2 in $10^{6}$ & $\begin{array}{l}L P L \\
A P O C 2 \\
\text { ApoAV, GPIHBP1 } \\
\text { LMF1 }\end{array}$ & $\uparrow \uparrow$ chylomicrons and VLDL-C \\
\hline Tangier disease (analphalipoproteinaemia) & 1 in $10^{6}$ & $A B C A 1$ & $\downarrow \downarrow$ HDL-C \\
\hline Familial LCAT deficiency & 1 in $10^{6}$ & LCAT & $\downarrow$ HDL-C \\
\hline
\end{tabular}

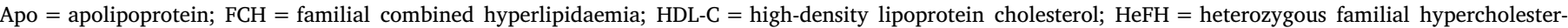

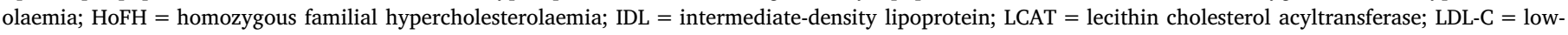
density lipoprotein cholesterol; VLDL = very low-density lipoprotein cholesterol.

treatment with a statin or, if dominated by high TGs, a fibrate; often a combination of a statin and a fibrate may be needed.

\subsubsection{Genetic causes of hypertriglyceridaemia}

Although the genetic aetiology for HTG seems to be very complex, recent data have extended our genetic understanding of HTG, in particular that of chylomicronaemia [37,226,377]. Moderate elevation of TG levels (between 2.0 and $10.0 \mathrm{mmoL} / \mathrm{L}$ ) is caused by the polygenic effect of multiple genes influencing both VLDL production and removal. Monogenic severe HTG causes chylomicronaemia, pancreatitis, and lipid deposits. Thus far, mutations in six genes ( $L P L$, apoC2, apoA5, $L M F 1$, GPIHBP1, and GPD1) with monogenic effects have been recognized to lead to severe elevation of serum TGs due to disruption of

\section{Table 12}

Dutch Lipid Clinic Network diagnostic criteria for familial hypercholesterolaemia.

\begin{tabular}{|c|c|}
\hline Criteria & Points \\
\hline \multicolumn{2}{|l|}{ 1) Family history } \\
\hline $\begin{array}{l}\text { First-degree relative with known premature (men aged }<55 \text { years; } \\
\text { women }<60 \text { years) coronary or vascular disease, or first-degree } \\
\text { relative with known LDL-C above the } 95 \text { th percentile }\end{array}$ & 1 \\
\hline $\begin{array}{l}\text { First-degree relative with tendinous xanthomata and/or arcus cornealis, } \\
\text { or children aged }<18 \text { years with LDL-C above the 95th percentile } \\
\text { 2) Clinical history }\end{array}$ & 2 \\
\hline $\begin{array}{l}\text { Patient with premature (men aged }<55 \text { years; women }<60 \text { years) } \\
\text { CAD }\end{array}$ & 2 \\
\hline $\begin{array}{l}\text { Patient with premature (men aged }<55 \text { years; women }<60 \text { years) } \\
\text { cerebral or peripheral vascular disease }\end{array}$ & 1 \\
\hline \multicolumn{2}{|l|}{ 3) Physical examination } \\
\hline Tendinous xanthomata & 6 \\
\hline Arcus cornealis before age 45 years & 4 \\
\hline \multicolumn{2}{|l|}{ 4) LDL-C levels (without treatment) } \\
\hline LDL-C $\geq 8.5 \mathrm{mmoL} / \mathrm{L}(\geq 325 \mathrm{mg} / \mathrm{dL})$ & 8 \\
\hline LDL-C $6.5-8.4 \mathrm{mmoL} / \mathrm{L}(251-325 \mathrm{mg} / \mathrm{dL})$ & 5 \\
\hline LDL-C 5.0-6.4 mmoL/L (191-250 mg/dL) & 3 \\
\hline LDL-C $4.0-4.9 \mathrm{mmoL} / \mathrm{L}(155-190 \mathrm{mg} / \mathrm{dL})$ & 1 \\
\hline \multicolumn{2}{|l|}{ 5) DNA analysis } \\
\hline Functional mutation in the $L D L R, a p o B$, or PCSK9 genes & 8 \\
\hline \multicolumn{2}{|c|}{$\begin{array}{l}\text { Choose only one score per group, the highest applicable; diagnosis is based on the total } \\
\text { number of points obtained }\end{array}$} \\
\hline \multicolumn{2}{|l|}{ A 'definite' FH diagnosis requires $>8$ points } \\
\hline \multicolumn{2}{|l|}{ A 'probable' $\mathrm{FH}$ diagnosis requires $6-8$ points } \\
\hline A 'possible' FH diagnosis requires $3-5$ points & \\
\hline
\end{tabular}

$\mathrm{CAD}=$ coronary artery disease; $\mathrm{FH}=$ familial hypercholesterolaemia; LDL$\mathrm{C}=$ low-density lipoprotein cholesterol; PCSK9 $=$ proprotein convertase subtilisin/kexin type 9 .

${ }^{\text {a }}$ Exclusive of each other (i.e. maximum 6 points if both are present). the chylomicron removal pathways. These mutations are inherited as autosomal recessive traits and are rare. The profound defect in the catabolism of chylomicrons and VLDL results in chylomicronaemia and TG levels $>11.2 \mathrm{mmoL} / \mathrm{L}$ ( $>1000 \mathrm{mg} / \mathrm{dL}$ ), with turbid and milky serum. Severe HTG is seen in patients who are homozygous or compound heterozygous for mutations of the enzyme LPL, and in other genes linked to the catabolism of TG-rich lipoproteins. Heterozygous carriers of these same gene mutations commonly express moderate elevations of serum TG levels that expose them to increased CVD risk [378]. Recently, gene therapy for LPL deficiency has been developed and tested in clinical trials [379], and the alipogene tiparvovec was approved by the EMA in 2013. However, this therapy is no longer available. A gain-of-function mutation in apoC3 that leads to high ApoC-III levels can also cause severe HTG by inhibiting the activity of LPL, whereas loss-of-function mutations are associated with a favourable lipid profile with low TG levels [380]. These findings have raised the possibility of ApoC-III being a novel lipid drug target.

9.1.4.1. Action to prevent acute pancreatitis in severe hypertriglyceridaemia. The risk of pancreatitis is clinically significant if TGs are $>10 \mathrm{mmoL} / \mathrm{L}(880 \mathrm{mg} / \mathrm{dL})$, particularly when occurring in association with familial chylomicronaemia, and actions to prevent acute pancreatitis are mandatory [381,382]. Notably, HTG is the cause of $\sim 10 \%$ of all cases with pancreatitis, and patients can develop pancreatitis even when their TG concentration is $5-10 \mathrm{mmoL} / \mathrm{L}$ (440-880 mg/dL). Recent data from a prospective cohort study reported that the risk of acute pancreatitis increased significantly over the quartiles of serum TGs, highlighting the fact that, as a risk factor, serum TGs may have been underestimated [383]. Any factor that increases VLDL production can aggravate the risk of pancreatitis, with alcohol consumption being the most common contributing factor. Either a patient should be admitted to hospital if symptomatic, or careful and close follow-up of the patient's TG values should be undertaken. Restriction of calories and fat content (10-15\% recommended) in the diet, and alcohol abstinence are obligatory. Fibrate therapy (fenofibrate) should be initiated, with n-3 fatty acids (2-4 g/day) as adjunct therapy. Lomitapide may also be considered in severe cases [37]. In patients with DM, insulin therapy should be initiated to achieve good glycaemic control. In general, a sharp decrease of TG values is seen within 2-5 days. In the acute setting, plasmapheresis is able to rapidly lower TG levels [384]. Volanesorsen has been recently approved by the EMA as an adjunct to diet in adult patients with genetically confirmed FCS who are at high-risk for pancreatitis. 
Box 6

Management of dyslipidaemia in women

Statin treatment is recommended for primary prevention of ASCVD in high-risk women [34,35].

Statins are recommended for secondary prevention in women with the same indications and goals as in men [34,35].

Lipid-lowering drugs should not be given when pregnancy is planned, during pregnancy, or during the breastfeeding period. However, for severe FH patients, bile acid sequestrants (which are not absorbed) and/or LDL apheresis may be considered.

ASCVD = atherosclerotic cardiovascular disease; FH = familial hypercholesterolaemia; LDL = low-density lipoprotein.

\subsubsection{Other genetic disorders of lipoprotein metabolism}

Sometimes patients are encountered with extremely low levels of LDL-C or HDL-C. The most common form of genetic hypolipidaemia is hypobetalipoproteinaemia, which is dominantly inherited and often due to truncation of ApoB. Serum LDL-C is typically between 0.5 and $1.5 \mathrm{mmoL} / \mathrm{L}(20-60 \mathrm{mg} / \mathrm{dL})$. A more profound deficiency of ApoB occurs in abetalipoproteinaemia when steatorrhoea, and neurological or other complications require specialist treatment. Almost absent levels of HDL-C occur in Tangier disease (analphalipoproteinaemia) and very low levels of HDL-C occur in lecithin cholesterol acyltransferase (LCAT) deficiency. Both these conditions are associated with distinct clinical syndromes and require specialist investigation. Very high levels of HDL$\mathrm{C}$ are detected in patients with CETP deficiency. In the heterozygous form, levels of $2.0-2.3 \mathrm{mmoL} / \mathrm{L}(80-90 \mathrm{mg} / \mathrm{dL}$ ) are typically observed, and levels $\geq 5 \mathrm{mmoL} / \mathrm{L}$ ( $\geq 200 \mathrm{mg} / \mathrm{dL}$ ) are observed in homozygotes. This is not associated with atherosclerotic disease and may be associated with reduced risk.

Lysosomal acid lipase deficiency or cholesterol ester storage disease (in children with Wolman disease) is a rare cause (recessive transmission) of elevated LDL-C and low HDL-C, accompanied by hepatomegaly and microvesicular hepatosteatosis. Statin treatment does decrease LDL-C levels and could therefore prevent ASCVD in these patients, but it cannot stop the progression of liver damage. Treatment with a PCSK9 inhibitor may lead to an even greater overload of lysosomes [385]. Enzyme replacement therapy with sebelipase alfa might offer a treatment solution in the near future [386].

\subsection{Women}

Few randomized trials of statin therapy have reported independently significant CV benefits in women [387,389], chiefly because women have not been adequately represented in statin trials.

\subsubsection{Effects of statins in primary and secondary prevention}

There has previously been controversy over whether statins are effective for primary prevention in women. Using published data, a 2013 Cochrane analysis showed that statin therapy reduced all-cause mortality, vascular events, and revascularizations in primary prevention, and the proportional effects in women were similar to those in men [213]. The CTT collaboration has provided a more complete assessment of the evidence through a comprehensive analysis of IPD from 22 trials of statins vs. control and five trials of more-vs. less-intensive statin therapy [35]. Overall, 46675 (27\%) of 174149 participants were women, and after adjustment for non-gender differences, the proportional reductions per mmol/L reduction in LDL-C in major vascular events, major coronary events, coronary revascularization, and stroke were similar in women and men [35].

\subsubsection{Non-statin lipid-lowering drugs}

Definitive evidence of the cardioprotective effects of non-statin drugs that lower LDL-C is now available, and the beneficial effects are similar in both women and men. In the IMPROVE-IT study [33], the relative benefit of adding ezetimibe to simvastatin was similar in women and men [33]. In the ACCORD lipid study, there was no evidence that fenofibrate added to the effects of simvastatin in patients with T2DM [306], but an analysis of the FIELD study showed consistent CV event reduction in both women and men [389]. Several outcome trials assessing the effects of adding a PCSK9 inhibitor to high-intensity statin therapy have now been reported, with similar proportional reductions in major vascular events in women and men [120,286,290].

\subsubsection{Hormone therapy}

Currently prescribed third-generation, low-dose oestrogen-progestin oral contraceptives do not appear to increase adverse coronary events [390] and can be used, after baseline lipid profile assessment, in women with acceptable TC levels. In contrast, alternative contraceptive measures should be recommended in women with hypercholesterolaemia [LDL-C $>4 \mathrm{mmoL} / \mathrm{L}(>160 \mathrm{mg} / \mathrm{dL})$ ] or with multiple risk factors, and in those at high-risk of thrombotic events [391]. Oestrogen replacement therapy, despite some favourable effects on lipid profiles, has not been demonstrated to reduce $\mathrm{CV}$ risk and cannot be recommended for CVD prevention in women [392]. No lipid-lowering drugs should be administered during pregnancy and the period of breastfeeding because data on possible adverse effects are lacking. However, bile acid sequestrants may be considered.

Box 6 lists the main measures in the management of dyslipidaemia in women.

\subsection{Older people}

The proportion of older people (defined herein as those aged $>65$ years) in society is increasing and, as a consequence, $>80 \%$ of individuals who die from CVD are $>65$ years of age. The proportion of patients with MI $>85$ years of age has increased several-fold [393].

Box 7

Summary of dyslipidaemia in metabolic syndrome and type 2 diabetes mellitus

Dyslipidaemia represents a cluster of lipid and lipoprotein abnormalities, including elevation of both fasting and post-prandial TG, ApoB, and small dense LDL, and low HDL-C and ApoA1 levels.

Non-HDL-C or ApoB are good markers of TRLs and remnants, and are a secondary objective of therapy. Non-HDL-C $<2.6 \mathrm{mmoL} / \mathrm{L}(<100$ $\mathrm{mg} / \mathrm{dL})$ and $\mathrm{ApoB}<80 \mathrm{mg} / \mathrm{dL}$ are desirable in those at high-risk, and non-HDL-C $<2.2 \mathrm{mmoL} / \mathrm{L}(<85 \mathrm{mg} / \mathrm{dL})$ and ApoB $<65 \mathrm{mg} / \mathrm{dL}$ in those at very high-risk. For those at very high-risk with recurrent ASCVD events, a goal of non-HDL-C $<1.8 \mathrm{mmoL} / \mathrm{L}(<70 \mathrm{mg} / \mathrm{dL})$ and ApoB $<55 \mathrm{mg} / \mathrm{dL}$ may be considered.

Atherogenic dyslipidaemia is one of the major risk factors for CVD in people with type 2 diabetes, and in people with abdominal obesity and insulin resistance or impaired glucose tolerance.

Apo = apolipoprotein; ASCVD = atherosclerotic cardiovascular disease; CVD = cardiovascular disease; HDL-C = high-density lipoprotein cholesterol; LDL-C = low-density lipoprotein cholesterol; TG = triglyceride; TRLs = triglyceride-rich lipoproteins. 
A meta-analysis of observational studies has shown that higher TC is associated with increased CAD mortality at all ages [62,394]. However, since the absolute risk of CAD is higher in older persons, the associated absolute increase in risk for a given increment in TC is larger with increasing age [217].

\subsubsection{Effects of statins in primary and secondary prevention}

The use of statin therapy declines with increasing age, reflecting differences in both prescription and compliance [395,396]. This trend is even more prominent among older patients who do not have evidence of occlusive vascular disease [396]. One explanation for this pattern may be uncertainty about the effects of statins in older people due to the relatively small number of people aged $>75$ years who have been included in statin trials $[233,397,398]$. The CTT collaboration recently provided a comprehensive assessment of the randomized evidence on the effects of statin therapy at different ages [217]. Among 186854 participants in 28 trials, $14483(8 \%)$ were aged $>75$ years at randomization. Overall, statin therapy produced a $21 \%$ relative reduction in major vascular events (relative risk $0.79,95 \%$ CI $0.77-0.81$ ) per 1.0 $\mathrm{mmoL} / \mathrm{L}$ reduction in LDL-C, and there was direct evidence of benefit among those aged $>75$ years. The relative reduction in major vascular events was similar, irrespective of age, among patients with pre-existing vascular disease, but appeared smaller among older individuals not known to have vascular disease. Therefore, the available evidence from trials indicates that statin therapy produces significant reductions in major vascular events irrespective of age. However, there is less direct evidence of benefit among patients aged $>75$ years who do not already have evidence of occlusive vascular disease, and this limitation is currently being addressed by the STAtin therapy for Reducing Events in the Elderly (STAREE) trial in Australia.

\subsubsection{Adverse effects, interactions, and adherence}

The safety and adverse effects of statins are a matter of special concern in older adults because they often have comorbidities, take multiple medications, and have altered pharmacokinetics and pharmacodynamics. Statin-drug interactions are a concern, primarily because of their potential to increase muscle-related statin-associated adverse effects such as myalgia without CK elevation, myopathy with CK elevation, and the rare but serious rhabdomyolysis. It is recommended that a statin is started at a low dose if there is significant renal impairment and/or the potential for drug interactions, and then titrated upwards to achieve LDL-C treatment goals.

The recommendations for the treatment of dyslipidaemias in older people are shown below.
[399]. Despite significant advantages in the management strategies that lessen atherosclerotic CVD risk factors, CVD has remained the leading cause of morbidity and mortality in patients with T2DM. The good news is that fatal CVD outcomes have declined significantly in both T1DM and T2DM between 1998 and 2014 [400]. DM itself is an independent risk factor for CVD and is associated with a higher risk of CVD, even more so in women. The difference in CVD risk between individuals with and without DM has narrowed substantially over the last few decades [401], and there are strong associations between DM and vascular outcomes [402,403]. Recent data indicate that DM per se increases CVD risk about two-fold on average, but the risk is subject to wide variation depending on the population and current aggressive prophylactic therapy $[401,404]$. Importantly, those with DM and CAD are at substantially higher CVD risk for future events. In T2DM, the risk of ASCVD is strongly determined by the presence of target organ damage-including nephropathy (microalbuminuria), neuropathy, or retinopathy-with the risks increasing in relation to the number of conditions present [405]. Hypertension, dyslipidaemia, abdominal obesity, and non-alcoholic fatty liver disease (NAFLD) commonly coexist with T2DM and further aggravate the risk, which is highest in people with T2DM and multiple cardiometabolic risk factors [406-408]. Importantly, DM confers excess mortality risk following ACS despite modern therapies, highlighting the poor prognosis of coronary patients with T2DM and the need for intensive therapy [409].

How to capture the extra risk beyond the traditional risk factors in clinical practice is a debated issue. A practical approach is that if one component is identified, a systematic search should be made for the others [410].

\subsubsection{Specific features of dyslipidaemia in insulin resistance and type 2 diabetes mellitus}

Diabetic dyslipidaemia is a cluster of plasma lipid and lipoprotein abnormalities that are metabolically interrelated. The increase in large VLDL particles in T2DM initiates a sequence of events that generates atherogenic remnants, small dense LDL, and small TG-rich dense HDL particles [411]. These components are not isolated abnormalities but are closely linked to each other. Both LDL and HDL particles show variable compositional changes that are reflected in their functions. Notably, ApoC-III levels are increased in people with T2DM [412]. High ApoC-III concentrations prevent the clearance of both TRLs and remnants, resulting in prolonged residence times of these particles in the circulation $[413,414]$. In fact, the defective catabolism of TRLs seems to be a more important contributor to the elevation of plasma TGs than the increased production rate leading to an excess of remnant particles.

Recommendations for the treatment of dyslipidaemias in older people (aged $>65$ years).

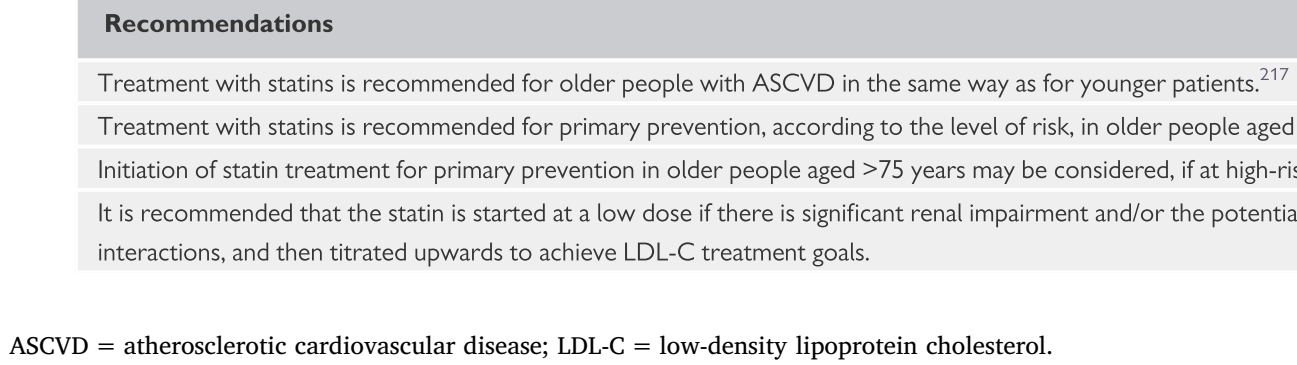

ASCVD = atherosclerotic cardiovascular disease; LDL-C = low-density lipoprotein cholesterol.

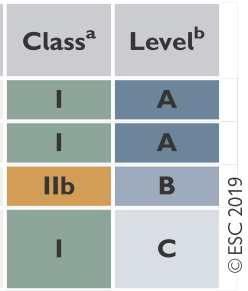

${ }^{a}$ Class of recommendation.

${ }^{\mathrm{b}}$ Level of evidence.

\subsection{Diabetes and metabolic syndrome}

The number of people with DM will increase from $\sim 415$ million today up to 550 million by 2030 , but the situation may get even worse
Together, TRL remnants, small dense LDL, and small dense HDL comprise the atherogenic lipid profile, which is also characterized by an increase in $\mathrm{ApoB}$ concentration due to an increased number of ApoBcontaining particles. Importantly, TRLs-including chylomicrons, 
VLDL, and their remnants-carry a single ApoB molecule, also like LDL particles. Therefore, the malignant nature of diabetic dyslipidaemia is not always revealed by the lipid measures used in clinical practice, as LDL-C levels may remain within the normal range. It may be better revealed by non-HDL-C levels [415]. Elevation of TGs or low HDL-C levels in the fasting or post-prandial state is seen in about one-half of all people with T2DM [416,417], and is also often present in people with abdominal adiposity, insulin resistance or impaired glucose tolerance [413].

Box 7 summarizes dyslipidaemia in MetS and T2DM.

\subsubsection{Evidence for lipid-lowering therapy}

9.4.2.1. Low-density lipoprotein cholesterol. LDL-C is the primary target of lipid-lowering therapy in patients with DM. Trials specifically performed in people with T2DM, as well as subsets of individuals with DM in major statin trials, have consistently demonstrated significant benefits of statin therapy on CVD events in people with T2DM [418]. Statin therapy reduces the 5 year incidence of major CVD events by $23 \%$ per $1 \mathrm{mmoL} / \mathrm{L}$ reduction in LDL-C, regardless of the initial LDL-C level or other baseline characteristics based on metaanalysis [418]. The CTT meta-analysis further indicates that people with T2DM will have a relative risk reduction that is comparable to that seen in non-diabetic patients; however, being at higher absolute risk, the absolute benefit will be greater, resulting in a lower number needed to treat (NNT). Thus, statin therapy is the first-line treatment for LDL-C lowering and for the reduction of CVD burden [419].

Ezetimibe lowers LDL-C by $\sim 24 \%$ and, when added to statin therapy, decreases the risk of major vascular events [33]. The relative risk reduction in major vascular events is proportional to the absolute degree of LDL-C lowering and consistent with the relationship seen for statins. The subset of patients with DM in IMPROVE-IT had, as expected, a higher rate of major vascular events than patients without DM (46 vs. $31 \% 7$ year Kaplan-Meier rate in the placebo arm). Ezetimibe appeared particularly efficacious in patients with DM, with a relative risk reduction of $15 \%$ (95\% CI 6-22\%) and an absolute risk reduction of 5.5\% [299].

The mAb PCSK9 inhibitors evolocumab and alirocumab lower LDL$\mathrm{C}$ levels by $\sim 60 \%$ and, when added to statin therapy, decrease the risk of major vascular events [119]. In the FOURIER study, the relative risk reduction for major vascular events was similar in patients with and without DM; however, given the higher baseline risk in patients with $\mathrm{DM}$, the absolute risk reductions tended to be greater in patients with DM $(2.7 \%$ absolute decrease in major vascular events over 3 years) [297]. Of note, the achieved LDL-C in the evolocumab arm was 0.8 $\mathrm{mmoL} / \mathrm{L}$. The same benefits were also recently demonstrated for diabetic patients after ACS in the ODYSSEY trial [420].

Recent studies have suggested an increased incidence of DM in patients treated with statins [247]. These observations have been seen in Mendelian randomization studies and in clinical trials, although the effect appears greatest in patients already at high risk for DM (e.g. those with pre-diabetes). These observations should not lessen our attention to the treatment of patients, as the overall benefits in $\mathrm{CV}$ event reduction remain and greatly outweigh the increased incidence of DM. In RCTs, neither ezetimibe nor the PCSK9 inhibitors have been reported to increase the risk of DM [297].

9.4.2.2. Triglycerides and high-density lipoprotein cholesterol. Lifestyle modification provides the first option to improve atherogenic dyslipidaemia due to its multifaceted effects. Weight loss is, in most cases, the most effective measure since it is associated with very pronounced effects on plasma TG and HDL levels, together with a modest decrease in TC and LDL-C levels. Moderate-to-heavy aerobic exercise is also associated with improvement of the plasma lipid profile by reducing TG levels and increasing HDL-C concentrations. In relation to diet composition, besides the need to eliminate trans fat, the available evidence supports the reduction of saturated fat intake and its substitution with unsaturated fat, as well as the replacement of a major proportion of refined starchy foods and simple sugars with fibrerich foods like fruits, vegetables, and wholegrains [179].

The clinical benefits achieved by the treatment of high TG and low HDL-C levels (frequently seen with DM) are still a matter of debate, as the effects of fenofibrate therapy on the major outcome (MACE) remained negative in both the FIELD and the ACCORD studies performed in T2DM cohorts [306,307]. In a post hoc analysis of the FIELD study, fenofibrate reduced CVD events by $27 \%$ in those with elevated TGs $[\sim 2.3 \mathrm{mmoL} / \mathrm{L}(200 \mathrm{mg} / \mathrm{dL})]$ and increased HDL-C levels $(\mathrm{NNT}=23)$ [416]. The ACCORD trial confirmed the following: patients who had both TG levels in the higher third $[\sim 2.3 \mathrm{mmoL} / \mathrm{L}(200 \mathrm{mg} / \mathrm{dL})]$ and an HDL-C level in the lower third $[\leq 0.4 \mathrm{mmoL} / \mathrm{L}(\leq 34 \mathrm{mg} / \mathrm{dL})]$, representing $17 \%$ of all participants, appeared to benefit from the addition of fenofibrate to simvastatin [306].

Recently, post-trial follow-up of the ACCORD lipid trial participants reported the beneficial effect of fenofibrate in people with HTG and low HDL-C levels at baseline [421]. Consistent with these findings, a metaanalysis of fibrates in the prevention of CVD in 11590 people with T2DM showed that fibrates significantly reduced the risk of non-fatal MI by $21 \%$, but had no effect on the risk of overall mortality or coronary mortality [422]. In CV outcome trials of fibrates, the risk reduction has appeared to simply be proportional to the degree of nonHDL-C lowering [50].

Overall, available data indicate that diabetic patients with atherogenic dyslipidaemia may derive clinical benefits from TG-lowering therapy as an add-on to statin treatment [354]. The ongoing PROMINENT trial is exploring the efficacy of pemafibrate, a new selective PPAR- $\alpha$ modulator, in reducing CVD outcomes in $\sim 10000$ diabetic patients with atherogenic dyslipidaemia on a statin [317,423].

There are limited data on the impacts on CVD of adding omega-3 fatty acids to statin therapy in patients with high plasma TG levels who are treated with statins. The REDUCE-IT trial examined the effects of icosapent ethyl $2 \mathrm{~g}$ b.i.d. on CV events in 8179 high-risk patients with HTG who were taking a statin. Over a median of 4.9 years, there was a significant $(P<0.001) 25 \%$ reduction in the composite primary outcome of CV death, non-fatal MI, non-fatal stroke, coronary revascularization, or unstable angina, corresponding with an absolute reduction of $4.8 \%$, which was offset by a $1 \%$ increased absolute risk of hospitalization for atrial fibrillation or flutter [194]. The STRENGTH trial is investigating the effect of omega-3 fatty acids, in addition to a statin, in individuals with HTG and low HDL-C levels who are at high-risk for CVD. The ASCEND trial was a randomized $2 \times 2$ factorial design study of aspirin and omega-3 fatty acid supplements for the primary prevention of CV events in people with DM, but not specifically with HTG. Among 15480 people randomized to omega- 3 fatty acid supplements vs. placebo over a mean follow-up of 7.4 years, there was no significant effect (HR 0.97, 95\% CI 0.87-1.08) on serious vascular events [nonfatal MI, non-fatal stroke, transient ischaemic attack (TIA), or vascular death] [329,424-426].

\subsubsection{Type 1 diabetes mellitus}

T1DM is associated with high CVD risk, in particular in patients with microalbuminuria and renal disease [427]. Conclusive evidence supports the proposition that hyperglycaemia accelerates atherosclerosis. Emerging evidence highlights the frequent coexistence of MetS with T1DM, resulting in the so-called double diabetes increasing CVD risk [428].

The lipid profile in T1DM patients with good glycaemic control is 'supernormal', and is characterized by subnormal TG and LDL-C levels, whereas HDL-C levels are usually within the upper normal range or slightly elevated. This is explained by subcutaneous administration of insulin that increases LPL activity in adipose tissue and skeletal muscle, and consequently the turnover rate of VLDL particles [429]. However, there are potentially atherogenic changes in the compositions of both HDL and LDL particles. 
Consistent data have demonstrated the efficacy of statins in preventing $\mathrm{CV}$ events and reducing CV mortality in patients with DM, with no evidence of sex differences [430,431]. A meta-analysis including 18686 patients with DM demonstrated that a statin-induced reduction of LDL-C yielded a $9 \%$ reduction in all-cause mortality and a $21 \%$ reduction in the incidence of major CV events per $1.0 \mathrm{mmoL} / \mathrm{L}(40 \mathrm{mg} / \mathrm{dL})$ lower LDL cholesterol [418]. Similar benefits were seen in patients with T1DM and T2DM. In diabetic patients with ACS, intensive statin treatment led to a reduction in all-cause mortality and CV death, and contributed to a reduction in atheroma progression [432].

\subsubsection{Management of dyslipidaemia for pregnant women with diabetes}

In both T1DM and young-onset T2DM patients, there is a paucity of evidence to indicate the age at which statin therapy should be initiated. To guide an approach, statins are not indicated in pregnancy [433], and should be avoided in both T1DM and T2DM individuals who are planning pregnancy. If diabetic individuals aged $\leq 30$ years have no evidence of vascular damage and, in particular, microalbuminuria, it seems reasonable to delay statin therapy in asymptomatic patients until the age of 30. Below this age, statin therapy should be managed on a case-by-case basis, taking into account the presence of microalbuminuria, end organ damage, and ambient LDL-C levels.

Recommendations for the treatment of dyslipidaemias in DM are shown in the table below.

Recommendations for the treatment of dyslipidaemias in diabetes mellitus.

\begin{tabular}{|c|c|c|}
\hline Recommendations & Class $^{a}$ & Level $^{\mathrm{b}}$ \\
\hline $\begin{array}{l}\text { In patients with } T 2 D M \text { at very-high } \text { risk }^{c} \text {, an } \\
L D L-C \text { reduction of } \geq 50 \% \text { from baseline and } \\
\text { an } L D L-C \text { goal of }<1.4 \mathrm{mmol} / \mathrm{L}(<55 \mathrm{mg} / \mathrm{dL}) \text { is } \\
\text { recommended. }{ }^{34,418,432}\end{array}$ & $\mathbf{I}$ & A \\
\hline $\begin{array}{l}\text { In patients with T2DM at high risk, }{ }^{c} \text { an } L D L-C \\
\text { reduction of } \geq 50 \% \text { from baseline and an LDL- } \\
C \text { goal of }<1.8 \mathrm{mmol} / \mathrm{L}(<70 \mathrm{mg} / \mathrm{dL}) \text { is } \\
\text { recommended. }{ }^{418}\end{array}$ & I & $\mathbf{A}$ \\
\hline $\begin{array}{l}\text { Statins are recommended in patients with } \\
\text { T1DM who are at high or very-high risk. }{ }^{\text {c }} 27\end{array}$ & I & $\mathbf{A}$ \\
\hline $\begin{array}{l}\text { Intensification of statin therapy should be con- } \\
\text { sidered before the introduction of combina- } \\
\text { tion therapy. }\end{array}$ & Ila & C \\
\hline $\begin{array}{l}\text { If the goal is not reached, statin combination } \\
\text { with ezetimibe should be considered. }{ }^{33,299}\end{array}$ & IIa & B \\
\hline $\begin{array}{l}\text { Statin therapy is not recommended in pre- } \\
\text { menopausal patients with diabetes who are } \\
\text { considering pregnancy or are not using } \\
\text { adequate contraception. }\end{array}$ & III & C \\
\hline $\begin{array}{l}\text { Statin therapy may be considered in both } \\
\text { T1DM and T2DM patients aged } \leq 30 \text { years } \\
\text { with evidence of end organ damage and/or an } \\
\text { LDL-C level }>2.5 \mathrm{mmol} / \mathrm{L} \text {, as long as preg- } \\
\text { nancy is not being planned. }\end{array}$ & Illb & C \\
\hline
\end{tabular}

LDL-C = low-density lipoprotein cholesterol; T1DM = type 1 diabetes mellitus; T2DM $=$ type 2 diabetes mellitus.

${ }^{\text {a }}$ Class of recommendation.

${ }^{\mathrm{b}}$ Level of evidence.

${ }^{\mathrm{c}}$ See Table 6.

\subsection{Patients with acute coronary syndromes and patients undergoing} percutaneous coronary intervention

Patients who present with ACS are at increased risk of experiencing recurrent $\mathrm{CV}$ events. For these patients, lipid management should be undertaken in the context of a comprehensive global risk reduction strategy including lifestyle adaptations, risk factor management, and the implementation of cardioprotective drug strategies. Ideally, patients should be signed up to cardiac rehabilitation programmes to enhance the control of lipid levels [434] and improve overall survival following ACS [435]. Despite the acknowledged clinical benefits of lowering LDLC in patients with ACS [436], attainment of LDL-C target values remains suboptimal in this very high-risk setting [437].

\subsubsection{Lipid-lowering therapy in patients with acute coronary syndromes}

LDL-C levels tend to decrease during the first days of ACS and therefore a lipid profile should be obtained as soon as possible after admission for ACS. Patients do not have to be fasting as this has little impact on LDL-C levels [100]. Lipid-lowering treatment should be initiated as early as possible to increase patient adherence after discharge. Lipid levels should be re-evaluated 4-6 weeks after ACS to determine whether treatment goals have been achieved and to check for any safety issues; the therapeutic regimen can then be adapted accordingly.

9.5.1.1. Statins. Data from RCTs and meta-analyses indicate that routine early use of high-intensity statin therapy is associated with rapid and sustained clinical benefits [438-442]. We recommend the initiation of high-intensity statin therapy in all statin-naïve ACS patients with no contraindication, regardless of initial LDL-C values; the treatment goal is to reach a 50\% LDL-C reduction from baseline and an LDL-C goal of $<1.4 \mathrm{mmoL} / \mathrm{L}$ ( $<55 \mathrm{mg} / \mathrm{dL}$ ). In those with recurrent events within 2 years while taking maximally tolerated statin therapy, a goal of $<1.0 \mathrm{mmoL} / \mathrm{L}$ ( $<40 \mathrm{mg} / \mathrm{dL}$ ) for LDL-C should be considered. The intensity of statin therapy should be increased in those patients receiving low- or moderate-intensity statin treatment at presentation, unless there is a definite history of intolerance to high-intensity statin therapy. The use of lower-intensity statin therapy should be considered in patients at increased risk of adverse effects with high-intensity statin therapy, such as in the elderly, patients diagnosed with hepatic or renal impairment, or in the case of a potential risk of drug-drug interactions with other essential concomitant therapies.

Regarding the timing of statin treatment initiation, the Statins Evaluation in Coronary Procedures and Revascularization (SECUREPCI) randomized, placebo-controlled trial recently assessed the impact of peri-procedural loading with atorvastatin [two loading doses of 80 $\mathrm{mg}$, before and $24 \mathrm{~h}$ after the planned percutaneous coronary intervention (PCI)] on MACE at 30 days in 4191 patients with ACS and planned invasive management [443]. All patients received atorvastatin $40 \mathrm{mg}$ per day starting $24 \mathrm{~h}$ after the second loading dose. The authors found no significant treatment benefit in the overall study population. In a pre-specified analysis, a significant $28 \%$ relative risk reduction in MACE was observed among patients who underwent PCI (65\% of all patients). The benefit was even more pronounced (46\% relative risk reduction) in a post hoc analysis including 865 ST-elevation MI (STEMI) patients undergoing reperfusion by primary PCI [443]. Based on current evidence, we recommend the initiation of high-intensity statin therapy during the first 1-4 days of hospitalization for the index ACS [438-442]. Moreover, pre-treatment (or loading dose for patients already on a statin) with a high-intensity statin should be considered in ACS patients with planned invasive management [443].

9.5.1.2. Ezetimibe. In the IMPROVE-IT trial, the addition of ezetimibe to simvastatin therapy provided an additional benefit (6.4\% relative risk reduction in the composite clinical endpoint) to post-ACS patients [33]. The clinical benefit of adding ezetimibe was consistent across patient subgroups [299,444] and also led to a reduction of total CV events [445], 
stroke [446], and rehospitalizations [447]. Patients at higher atherothrombotic risk [as assessed by the TIMI (Thrombolysis In Myocardial Infarction) risk score for secondary prevention] benefitted the most from the addition of ezetimibe [448]. In another randomized, open-label trial including 1734 patients with ACS, the addition of ezetimibe to moderate-intensity statin (pitavastatin $2 \mathrm{mg}$ ) therapy failed to improve outcomes overall, but did reduce the composite primary endpoint (death, MI, stroke, unstable angina, and ischaemia-driven revascularization) during 3.9-year follow-up in patients with increased intestinal absorption of cholesterol (as assessed by elevated levels of sitosterol) [449]; however, this finding requires further confirmation.

9.5.1.3. Proprotein convertase subtilisin/kexin type 9 inhibitors. In the FOURIER trial, which included 27564 patients with atherosclerotic CV disease, the addition of evolocumab to statin therapy (69\% high-intensity therapy) resulted in a $15 \%$ relative risk reduction of the composite primary endpoint throughout a 2.2 year follow-up. Results were consistent in the subgroup of patients with a history of MI $(81 \%$ of all patients) $[119,450]$. A subanalysis of the FOURIER trial showed that patients who achieved the lowest LDL-C values under PCSK9 treatment also had the lowest risk of future MACE [451]. In the ODYSSEY Outcomes trial, which included 18924 patients with recent ACS (1-12 months prior to enrolment, median 2.6 months), alirocumab added to statin therapy (89\% high-intensity therapy) also resulted in a $15 \%$ relative risk reduction in the primary composite endpoint and was associated with a $15 \%$ relative reduction in all-cause mortality throughout a 2.8 year follow-up [120]. No serious side effects or safety concerns were reported in these two large trials. The optimal timing of initiating PCSK9 inhibition after ACS and its impact on clinical outcomes remain to be determined. Regarding the timing of PCSK9 inhibitor treatment initiation, post hoc analyses from the FOURIER trial have shown that the closer to the event this is done, the better. Treatment initiation with PCSK9 inhibitors during the acute phase of ACS is under investigation in the EVOlocumab for Early Reduction of LDL-cholesterol Levels in Patients With Acute Coronary Syndromes (EVOPACS) trial [452]. Based on current evidence, we recommend the initiation of treatment with PCSK9 inhibitors in patients with ACS who do not reach their respective LDL-C goals (as outlined in Table 7) after 4-6 weeks of maximum tolerated statin and ezetimibe therapy. In patients who present with an ACS and whose LDL-C levels are not at goal, despite already taking a maximally tolerated statin dose and ezetimibe prior to the event, the addition of a PCSK9 inhibitor early after the event (during the hospitalization for the ACS event if possible) should be considered.

9.5.1.4. n-3 polyunsaturated fatty acids. Oral supplementation with highly purified n-3 PUFAs reduced mortality in survivors of MI in one study [Gruppo Italiano per lo Studio della Sopravvivenza nell'Infarto Miocardico-Prevenzione (GISSI-P)] but failed to affect clinical outcomes in subsequent trials using contemporary secondary prevention therapies. A recent meta-analysis of available RCTs showed no reduction in mortality, MI, or major vascular events associated with n-3 PUFAs, including the subgroup of patients with known CAD [453]. Therefore, routine treatment with n-3 PUFAs cannot be recommended.

9.5.1.5. Cholesteryl ester transfer protein inhibitors. In 2007, a large prospective study using the CETP inhibitor torcetrapib failed to show any clinical benefit in more than 15000 high-risk patients, and was potentially harmful [336]. The CETP inhibitors dalcetrapib (in $>30000$ patients with recent ACS [65]) and evacetrapib (in > 12000 high-risk patients [63]) were investigated in 2012 and 2017 , respectively. Neither clinical study was able to show any clinical benefit associated with CETP inhibitors [65]. More recently, the REVEAL study investigated anacetrapib in $>30000$ patients with atherosclerotic vascular disease and resulted in a lower incidence of MACE compared with placebo after 4 years, with no safety concerns [64]. However, this compound was not filed for marketing authorization.

\subsubsection{Lipid-lowering therapy in patients undergoing percutaneous coronary} intervention

In a meta-analysis of 13 randomized studies including 3341 patients who were planned to undergo PCI, pre-treatment with a high-dose statin (statin-naïve patients, 11 studies) or a high-dose statin loading dose reduced the risk of MACE (death, MI, or target vessel revascularization) by $44 \%$ both for peri-procedural $\mathrm{MI}$ and MACE at 30 days [454]. In all but one study, PCI was performed in the setting of stable angina or in a non-emergency setting in non-ST elevation ACS (NSTEACS). One of the studies that was included in the meta-analysis showed an improvement in coronary flow when primary PCI was used for the treatment of STEMI [455]. A routine strategy of either short pre-treatment or loading (on the background of pre-existing therapy) with a high-dose statin before PCI should be considered in elective PCI or NSTE-ACS [454,456,457].

In addition, pre-treatment with a statin has also been shown to reduce the risk of contrast-induced acute kidney injury after coronary angiography or intervention [458].

Recommendations for lipid-lowering therapy in patients with ACS and patients undergoing PCI are summarized below.

Recommendations for lipid-lowering therapy in very-high-risk patients with acute coronary syndromes.

\begin{tabular}{|c|c|c|}
\hline Recommendations & Class $^{a}$ & Level $^{b}$ \\
\hline $\begin{array}{l}\text { In all ACS patients without any contraindica- } \\
\text { tion or definite history of intolerance, it is rec- } \\
\text { ommended that high-dose statin therapy is } \\
\text { initiated or continued as early as possible, } \\
\text { regardless of initial LDL-C values. }{ }^{438,440,442}\end{array}$ & I & $\mathbf{A}$ \\
\hline $\begin{array}{l}\text { Lipid levels should be re-evaluated } 4-6 \text { weeks } \\
\text { after ACS to determine whether a reduction } \\
\text { of } \geq 50 \% \text { from baseline and goal levels of LDL- } \\
C<1.4 \mathrm{mmol} / \mathrm{L} \text { ( }<55 \mathrm{mg} / \mathrm{dL}) \text { have been } \\
\text { achieved. Safety issues need to be assessed at } \\
\text { this time and statin treatment doses adapted } \\
\text { accordingly. }\end{array}$ & Ila & C \\
\hline $\begin{array}{l}\text { If the LDL-C goal is not achieved after } 4-6 \\
\text { weeks with the maximally tolerated statin } \\
\text { dose, combination with ezetimibe is } \\
\text { recommended. }{ }^{33}\end{array}$ & I & B \\
\hline $\begin{array}{l}\text { If the LDL-C goal is not achieved after } 4-6 \\
\text { weeks despite maximal tolerated statin ther- } \\
\text { apy and ezetimibe, the addition of a PCSK9 } \\
\text { inhibitor is recommended. }{ }^{119,120}\end{array}$ & I & B \\
\hline $\begin{array}{l}\text { In patients with confirmed statin intolerance } \\
\text { or in patients in whom a statin is contraindi- } \\
\text { cated, ezetimibe should be considered. }\end{array}$ & Ila & C \\
\hline $\begin{array}{l}\text { For patients who present with an ACS and } \\
\text { whose LDL-C levels are not at goal, despite } \\
\text { already taking a maximally tolerated statin } \\
\text { dose and ezetimibe, the addition of a PCSK9 } \\
\text { inhibitor early after the event (during hospital- } \\
\text { ization for the ACS event if possible) should } \\
\text { be considered. }\end{array}$ & Ila & C \\
\hline
\end{tabular}

ACS = acute coronary syndrome; LDL-C = low-density lipoprotein cholesterol; PCSK9 $=$ proprotein convertase subtilisin/kexin type 9 .

a Class of recommendation.

b Level of evidence. 
Recommendations for lipid-lowering therapy in very-high-risk patients undergoing percutaneous coronary intervention.

\begin{tabular}{|l|l|l|}
\hline Recommendations & Class $^{\mathbf{a}}$ & Level $^{\mathbf{b}}$ \\
\hline $\begin{array}{l}\text { Routine pre-treatment or loading (on a back- } \\
\text { ground of chronic therapy) with a high-dose }\end{array}$ & Ila \\
statin should be considered in patients under- \\
going PCl for an ACS or elective $\mathrm{PCl}^{443,454,456}$
\end{tabular}

ACS $=$ acute coronary syndrome; PCI = percutaneous coronary intervention.

${ }^{\text {a }}$ Class of recommendation.

${ }^{\mathrm{b}}$ Level of evidence.

\subsection{Stroke}

Stroke has a heterogeneous aetiology, including cardiac thromboembolism [often associated with atrial fibrillation, but also of uncertain source (embolic stroke of undetermined source)], carotid artery and proximal aortic atherosclerosis and thromboembolism, small-vessel CVD, and intracranial haemorrhage (including intracerebral and subarachnoid haemorrhage). Dyslipidaemia may play a variable role in the pathogenesis of stroke according to the particular aetiology. The relationship between dyslipidaemia and atherothrombotic events, including ischaemic stroke and TIA, is well recognized, while the association of dyslipidaemia with other types of stroke is uncertain. Notwithstanding, concomitant control of other aetiological factors, such as hypertension, is of paramount importance.

Following ischaemic stroke or TIA, patients are at risk not only of recurrent cerebrovascular events, but also of other major CV events, including MI. Secondary prevention therapy with statins reduces the risk of recurrent stroke (by $12 \%$ per $\mathrm{mmol} / \mathrm{L}$ reduction in LDL cholesterol), MI, and vascular death $[459,460]$. Statin pre-treatment at TIA onset was associated with reduced recurrent early stroke risk in patients with carotid stenosis in a pooled data analysis, supporting as-early-aspossible initiation of statins after stroke [460-462]. Statin therapy may yield a small increase in the risk of haemorrhagic stroke, but the evidence regarding this risk is uncertain [34,36,251,252].

Recommendations for lipid-lowering therapy for the prevention of atherosclerotic cardiovascular disease events in patients with prior ischaemic stroke.

\begin{tabular}{l|l|l|}
\hline Recommendations & Class $^{\mathbf{a}}$ & Level $^{\mathbf{b}}$ \\
\hline Patients with a history of ischaemic stroke or & \\
TIA are at very high-risk of ASCVD, particu- \\
larly recurrent ischaemic stroke, so it is rec- \\
ommended that they receive intensive LDL-C- \\
lowering therapy.
\end{tabular}

ASCVD = atherosclerotic cardiovascular disease; LDL-C = low-density lipoprotein cholesterol; TIA $=$ transient ischaemic attack.

${ }^{\text {a }}$ Class of recommendation.

${ }^{\mathrm{b}}$ Level of evidence.

\subsection{Heart failure and valvular diseases}

9.7.1. Prevention of incident heart failure in coronary artery disease patients

Cholesterol lowering with statins reduces the incidence of HF in patients with CAD (stable CAD or a history of ACS) without previous HF; this has been shown consistently in RCTs that have compared statin vs. no statin treatment [463,464] as well as more-intensive vs. lessintensive statin therapy [465-468]. A large-scale meta-analysis of primary and secondary prevention RCTs with statins showed a modest (10\%) reduction in first non-fatal HF hospitalizations with statin treatment, with no effect on HF death within the limited RCT period [469]. There is no evidence that statins can prevent HF of nonischaemic origin.

\subsubsection{Chronic heart failure}

Two large RCTs [466,470] (mainly including patients with systolic $\mathrm{HF}$ ), as well as a meta-analysis of 24 RCTs, have shown no benefit of statin treatment on CV mortality or stroke [471]; a reduction in HF hospitalizations [218,471], as well as a small reduction in MI, was observed in a pooled analysis of the Controlled Rosuvastatin Multinational Trial in Heart Failure (CORONA) and GISSI-HF trials [472]. Based on current evidence, routine administration of statins in patients with $\mathrm{HF}$ without other indications for their use (e.g. CAD) is not recommended. Because there is no evidence of harm in patients on statin treatment after the occurrence of HF, there is no need for statin discontinuation for patients already on treatment.

There is no evidence regarding the effect of PCSK9 inhibition in patients with chronic HF. In the recent PCSK9 clinical outcomes trials, FOURIER [119] and ODYSSEY Outcomes [120], PCSK9 inhibition in patients with atherosclerotic CVD or after an ACS did not reduce the risk of HF hospitalization. In the BIOlogy Study to TAilored Treatment in Chronic Heart Failure (BIOSTAT-CHF) study of 2174 patients with worsening HF, multivariable analysis revealed a positive linear association between PCSK9 levels and the risk of mortality, and the composite of mortality and unplanned HF hospitalization [473]. Similarly, there was a negative association between LDLR levels and mortality, indicating a potential relationship between the PCSK9-LDLR axis and outcomes among patients with HF that requires further investigation $[473,474]$.

Treatment with n-3 PUFAs $1 \mathrm{~g}$ o.d. may confer a small benefit in patients with chronic HF, as shown by a significant $9 \%$ relative risk reduction for mortality in the GISSI-HF RCT [475].

\subsubsection{Valvular heart diseases}

Aortic stenosis increases the risk of CV events and mortality, and frequently coexists with atherosclerotic CVD. Life-long high levels of LDL-C [476] and Lp(a) [477] have been associated with incident aortic valve stenosis and aortic valve calcification in genetic Mendelian randomization studies. Observational studies have suggested possible beneficial effects of intensive lipid lowering in slowing the progression of native valve aortic stenosis [478]. However, this has not been confirmed in RCTs [266,479-481], or in meta-analyses of observational and randomized trials [482,483]. Three modestly sized trials [479-481] and one large randomized trial (SEAS, which included 1873 patients treated with simvastatin $40 \mathrm{mg}$ plus ezetimibe $10 \mathrm{mg}$ or placebo) [266] failed to show a reduction in the clinical progression of aortic stenosis in patients with mild-to-moderate native valve aortic stenosis. In a post hoc analysis of the SEAS trial, the efficacy of lipidlowering therapy in impeding the progression of aortic stenosis increased with higher pre-treatment LDL-C levels and lower peak aortic jet velocity (i.e. milder stenosis at baseline) [484]. Similarly, a post hoc analysis of three RCTs, including patients without known aortic valve stenosis at baseline [Treating to New Targets (TNT), Incremental Decrease In End-points Through Aggressive Lipid-lowering (IDEAL), and Stroke Prevention by Aggressive Reduction in Cholesterol Levels (SPARCL)] showed no impact of high-dose vs. usual-dose statin therapy on the incidence of aortic valve stenosis [485]. In patients who underwent transcatheter aortic valve replacement, statin therapy was associated with improved outcomes in a small observational study [486].

Aortic valve sclerosis (calcification of the aortic leaflets without significant transvalvular pressure gradient) is associated with an increased risk of CAD even in the absence of increased risk profiles. Whether or not statins may be useful both for aortic valve disease and CAD progression in such patients warrants further investigation [487].

Recommendations for lipid-lowering therapy in patients with HF and valvular diseases are shown below. 
Recommendations for the treatment of dyslipidaemias in chronic heart failure or valvular heart diseases.

\begin{tabular}{|c|c|c|}
\hline Recommendations & Class $^{a}$ & Level $^{\mathrm{b}}$ \\
\hline $\begin{array}{l}\text { Initiation of lipid-lowering therapy is not rec- } \\
\text { ommended in patients with HF in the absence } \\
\text { of other indications for their use. }{ }^{466,470}\end{array}$ & III & A \\
\hline $\begin{array}{l}\text { Initiation of lipid-lowering treatment in } \\
\text { patients with aortic valvular stenosis without } \\
\text { CAD to slow progression of aortic valve } \\
\text { stenosis in the absence of other indications for } \\
\text { their use is not recommended. }{ }^{266,479-481}\end{array}$ & III & $\mathbf{A}$ \\
\hline
\end{tabular}

$\mathrm{CAD}=$ coronary artery disease $\mathrm{HF}=$ heart failure.

a Class of recommendation.

${ }^{\mathrm{b}}$ Level of evidence.

\subsection{Chronic kidney disease}

CKD is defined as abnormalities of kidney structure or function, present for $>3$ months, with implications for health. CKD is classified on the basis of the cause, GFR category, and category of albuminuria [488]. In the adult population, decreasing GFR is associated with increased CVD risk, independent of other CV risk factors [489-492]. There is an increased risk of both atherosclerotic vascular disease and structural heart disease [492]. Patients with CKD and established CVD have a much higher mortality rate compared with patients with CVD and normal renal function [493]. Therefore, patients with CKD are considered to be at high (stage $3 \mathrm{CKD}$ ) or very-high risk (stage 4-5 CKD or on dialysis) of CVD, and there is no need to use risk estimation models in these patients.

\subsubsection{Lipoprotein profile in chronic kidney disease}

In the initial stages of CKD, TG levels are specifically elevated and HDL-C levels are lowered. LDL subclasses display a shift to an excess of small dense LDL particles. Studies suggest that the kidney has a role in $\mathrm{Lp}$ (a) catabolism and that $\mathrm{Lp}$ (a) levels are increased in association with kidney disease. Such acquired abnormalities can be reversed by kidney transplantation or remission of nephrosis.

9.8.2. Evidence for risk reduction through statin-based therapy in patients with chronic kidney disease

In the Die Deutsche Diabetes Dialyse Studie (4D) trial, which involved 1200 patients with diabetes on haemodialysis, atorvastatin had no significant effect on risk of CVD [220]. Similar results were obtained in the AURORA (A study to evaluate the Use of Rosuvastatin in subjects On Regular haemodialysis: an Assessment of survival and cardiovascular events) trial, which involved 2776 patients on haemodialysis [221].

In the SHARP study [222], simvastatin and ezetimibe combination therapy reduced the risk for major atherosclerotic events (coronary death, MI, non-haemorrhagic stroke, or any revascularization) compared with placebo in persons with CKD stage 3A-5. The trial did not have sufficient power to separately assess the effects on the primary outcome in dialysis and non-dialysis patients. Although statin-based therapy is clearly effective in mild-to-moderate CKD, a major controversy that remained after the publication of the 4D, AURORA, and SHARP studies was whether statin therapy is effective in more advanced CKD, particularly dialysis patients. By combining data from the three CKD trials with other trials in the existing database, the CTT investigators found that, even after adjusting for the smaller LDL-C reductions achieved among patients with more advanced CKD and for differences in outcome definitions between dialysis trials, there was a trend towards smaller relative reductions per mmol/L reduction in LDL-
C in major atherosclerotic events as estimated GFR (eGFR) declines (with little evidence of benefit among dialysis patients) [214]. This diminution in relative risk reduction as GFR declines implies that, at least in non-dialysis patients, more intensive LDL-lowering regimens are required to achieve the same benefit.

\subsubsection{Safety of lipid management in patients with chronic kidney disease}

Safety issues and dose adjustments are important in advanced stages of CKD (stages 3-5), as adverse events are commonly dose-related and due to increased blood concentrations of compounds. Although it has been suggested that preference should be given to regimens and doses that have been shown to be beneficial in RCTs conducted specifically in such patients [494], the CTT meta-analysis makes clear that the goal-as in patients without CKD—should be to achieve the largest possible absolute reduction in LDL-C safely. Although there were no specific safety concerns raised by the $4 \mathrm{D}$, AURORA, or SHARP trials, statins metabolized via CYP3A4 may result in adverse effects due to drug-drug interactions and caution is required.

Based on the evidence for lipid management in patients with CKD, the Kidney Disease: Improving Global Outcomes (KDIGO) organization has developed an updated clinical practice guideline for lipid management in CKD [494]. In line with this, but with a focus on those patients at high or very-high risk for developing CVD, recommendations are summarized below.

Recommendations for lipid management in patients with moderate-to-severe (Kidney Disease Outcomes Quality Initiative stages 3-5) chronic kidney disease.

\begin{tabular}{|c|c|c|}
\hline Recommendations & Class $^{a}$ & Level $^{\text {b }}$ \\
\hline $\begin{array}{l}\text { It is recommended that patients with Kidney } \\
\text { Disease Outcomes Quality Initiative stage } 3-5^{c} \\
\text { CKD are considered to be at high or very-high risk } \\
\text { of ASCVD. }{ }^{489-493}\end{array}$ & I & $\mathbf{A}$ \\
\hline $\begin{array}{l}\text { The use of statins or statin/ezetimibe combina- } \\
\text { tion is recommended in patients with non-dialy- } \\
\text { sis-dependent stage } 3-5 \text { CKD. }{ }^{214,222,495,496}\end{array}$ & I & $\mathbf{A}$ \\
\hline $\begin{array}{l}\text { In patients already on statins, ezetimibe, or a sta- } \\
\text { tin/ezetimibe combination at the time of dialysis } \\
\text { initiation, continuation of these drugs should be } \\
\text { considered, particularly in patients with ASCVD. }\end{array}$ & Ila & C \\
\hline $\begin{array}{l}\text { In patients with dialysis-dependent CKD who } \\
\text { are free of ASCVD, commencement of statin } \\
\text { therapy is not recommended. }{ }^{220,221}\end{array}$ & III & $\mathbf{A}$ \\
\hline
\end{tabular}

ASCVD $=$ atherosclerotic cardiovascular disease; $\mathrm{CKD}=$ chronic kidney disease; eGFR = estimated glomerular filtration rate.

a Class of recommendation.

${ }^{\mathrm{b}}$ Level of evidence.

c Defined as eGFR $<60 \mathrm{ml} / \mathrm{min} / 1.73 \mathrm{~m}^{2}$ on two measurements more than 3 months apart.

\subsection{Transplantation}

Dyslipidaemias are very common in patients who have undergone heart, lung, liver, kidney, or allogenic haematopoietic stem cell transplantation, and predispose such patients to an increased risk of developing ASCVD and transplant arterial vasculopathy [497-501]. In patients with CKD undergoing renal transplantation, the risk of ASCVD may be determined, at least in part, by the increased risk resulting from CKD itself.

Immunosuppressive drug regimens may have adverse effects on lipid metabolism leading to increases in TC, VLDL, and TGs, and in the size and density of LDL particles. These effects vary with different 
immunosuppressive drugs [497,498,502-506].

The management of dyslipidaemias in transplant recipients is comparable to what is recommended for patients at high or very high ASCVD risk, although more attention is needed regarding the causes of the lipid disturbances and possible side effects due to drug-drug interactions (see Recommendations for low-density lipoprotein in solid organ transplant patients below).

The clinical effectiveness of statins in renal transplant patients is uncertain owing to a lack of randomized trials in this population. A systematic review of the benefits and harms of statins in patients with a functioning kidney transplant included 3465 patients, free of CHD, from 22 studies. Although the authors concluded that statins may reduce CV events, they also suggested a need for additional studies [253]. However, in patients with a functioning renal transplant at increased risk of CVD, it may be appropriate to extrapolate from the clear evidence of benefit from statin therapy, without safety concerns, in people with moderate reductions in GFR [214].

Several potential drug interactions must also be considered, especially with ciclosporin, which is metabolized through CYP3A4, and may increase systemic statin exposure and the risk of myopathy. Ciclosporin increases the blood levels of all statins.

Fluvastatin, pravastatin, pitavastatin, and rosuvastatin are metabolized through different CYP enzymes than the others and have less potential for interaction [507].

Tacrolimus is also metabolized by CYP3A4, but appears to have less potential for harmful interaction with statins than ciclosporin. Other drugs that influence CYP3A4 activity should be avoided if possible, and used with extreme caution in patients receiving both calcineurin inhibitors and statins.

For transplant patients with dyslipidaemia, ezetimibe could be considered as an alternative for patients unable to take a statin or added to the highest tolerated statin dose [507-509]. No outcome data are available for this drug, which should generally be reserved for secondline use. Ciclosporin can induce a $2-12$-fold increase in the ezetimibe level.

Care is required with the use of fibrates, as they can decrease ciclosporin levels and have the potential to cause myopathy. Extreme caution is required if fibrate therapy is planned in combination with a statin. Cholestyramine is not effective as monotherapy in heart transplant patients and has the potential to reduce absorption of immunosuppressants; this potential is minimized by separate administration.

Recommendations for low-density lipoprotein lowering in solid organ transplant patients.

\begin{tabular}{|c|c|c|}
\hline Recommendations & Class $^{\mathbf{a}}$ & Level $^{\mathrm{b}}$ \\
\hline $\begin{array}{l}\text { Statins should be considered as first-line agents in } \\
\text { transplant patients. Initiation should be at low } \\
\text { doses with careful uptitration and with caution } \\
\text { regarding potential drug-drug interactions, partic- } \\
\text { ularly for patients on ciclosporin. }{ }^{507}\end{array}$ & Ila & B \\
\hline $\begin{array}{l}\text { In patients who are intolerant of statins or those } \\
\text { with significant dyslipidaemia despite maximally } \\
\text { tolerated statin treatment, alternative or addi- } \\
\text { tional therapy with ezetimibe may be considered. }\end{array}$ & Ilb & C \\
\hline
\end{tabular}

${ }^{\text {a }}$ Class of recommendation.

${ }^{\mathrm{b}}$ Level of evidence.

\subsection{Peripheral arterial disease}

PAD encompasses all vascular sites, including carotid, vertebral, upper extremity, mesenteric, renal, and lower extremity arteries. The aorta is often included in the term [510]. PAD is a common manifestation of atherosclerosis and such patients are at elevated risk of coronary events, with PAD representing an independent risk factor for MI and CV death [510,511]. Patients with PAD are at very-high risk and should be managed according to the recommendations in Table 7 . Elevated CV risk has led to the inclusion of PAD among the list of 'risk equivalent' conditions, and therapeutic strategies of secondary prevention should be implemented [see Recommendations for lipid-lowering drugs in patients with peripheral arterial disease (including carotid artery disease) below]. Yet, despite the high CV morbidity and mortality risk, PAD patients are usually inadequately managed compared with CAD patients [511].

\subsubsection{Lower extremity arterial disease}

A low ABI (0.90) is diagnostic for lower extremity arterial disease (LEAD). Either a low (0.90) or high (1.40, related to stiffened arteries) $\mathrm{ABI}$ is predictive of CV morbidity and mortality. Lowering LDL-C levels reduces the risk of ischaemic CV events and worsening of claudication, while it also improves walking performance. As for cardiac events, a systematic review of 18 trials including 10000 patients, with cholesterol levels ranging from normal to elevated, reported that lipid-lowering therapy in people affected by atherosclerosis of the lower limbs was associated with a $20 \%$ reduction in total CV events, together with a non-significant $14 \%$ reduction of all-cause mortality [512]. In the Heart Protection Study, the need for non-coronary revascularization was reduced by $16 \%$ with statin therapy [513].

In addition to statins, PSCK9 inhibitors have also been shown to reduce $\mathrm{CV}$ events in $\mathrm{PAD}$ patients. In a pre-specified subgroup analysis of the FOURIER trial, evolocumab significantly reduced the primary endpoint in patients with PAD [514]. PAD had larger absolute risk reductions for the primary endpoint (3.5\% with PAD and 1.6\% without PAD). Evolocumab also reduced the risk of major adverse limb events by $42 \%$ in patients, with consistent effects in those with and without known PAD. In the FIELD trial, fenofibrate reduced the risk of amputations, particularly minor amputations without known large-vessel disease, probably through non-lipid mechanisms [515].

\subsubsection{Carotid artery disease}

While there are currently no randomized studies that have assessed whether lipid-lowering treatments reduce the incidence of $\mathrm{CV}$ events in patients enrolled on the basis of carotid atherosclerotic disease and without previous CV events, lipid-lowering therapy had reduced stroke in numerous studies. In a meta-analysis of RCTs enrolling $>90000$ patients, statin therapy did lead to a $21 \%$ reduction in the incidence of all strokes in different populations; this effect was mainly driven by the extent of LDL-C reduction [460].

\subsubsection{Retinal vascular disease}

Atherosclerotic changes of retinal arteries correlate with TC, LDL-C, TG, and apoB levels and also with CAD [516]. Fenofibrate reduces the progression of diabetic retinopathy $[517,518]$.

\subsubsection{Secondary prevention in patients with abdominal aortic aneurysm}

The presence of an abdominal aortic aneurysm represents a riskequivalent condition for $\mathrm{CAD}$ and is associated with age, male gender, personal history of atherosclerotic CVD, smoking, hypertension, and dyslipidaemia [519]; in contrast, diabetic patients are at decreased risk.

There are currently no available clinical trials on the reduction of CV risk with lipid-lowering therapy in patients affected by this condition. Systematic reviews [520], mostly based on retrospective nonrandomized studies, have reported that there is still inconclusive evidence that statin therapy reduces peri-operative CV morbidity and mortality. In an RCT comparing atorvastatin $20 \mathrm{mg}$ with placebo, the composite endpoint of cardiac death, MI, stroke, and unstable angina was significantly reduced in 100 patients undergoing vascular noncardiac surgery, including abdominal aortic aneurysm repair [521]. In 
another double-blind, placebo-controlled trial in 497 patients undergoing vascular surgery, peri-operative fluvastatin therapy $(80 \mathrm{mg} /$ day) was associated with an improvement in post-operative cardiac outcome [522].

\subsubsection{Renovascular atherosclerosis}

Lipid-lowering therapy has never been tested in an RCT in patients affected by renovascular atherosclerosis; however, a recent non-randomized population-based study showed that in patients older than 65 years of age with atherosclerotic renovascular disease raised the hypothesis that such treatment may yield cardiorenal benefits; the risk of a major cardiorenal composite endpoint (MI, stroke, HF, acute renal failure, dialysis, and death) was significantly lower in statin users than in non-users [523].

Recommendations for lipid-lowering drugs in patients with peripheral arterial disease (including carotid artery disease).

\begin{tabular}{l|l|l|}
\hline Recommendations & Class $^{\mathbf{a}}$ & Level $^{\mathbf{b}}$ \\
\hline In patients with PAD, lipid-lowering therapy, \\
including a maximum tolerated dose of statin, \\
plus ezetimibe or a combination with a PCSK9 \\
inhibitor if needed, is recommended to reduce \\
the risk of ASCVD events.
\end{tabular}

ASCVD = atherosclerotic cardiovascular disease; $\mathrm{PAD}=$ peripheral arterial disease; PCSK9 = proprotein convertase subtilisin/kexin type 9.

${ }^{a}$ Class of recommendation.

${ }^{\mathrm{b}}$ Level of evidence.

\subsection{Other special populations at risk of atherosclerotic cardiovascular} disease

In general, the effects of lowering LDL-C are determined by the absolute risk of ASCVD and the achieved reduction in LDL-C, so it is important to identify and treat all those at increased risk of ASCVD. There are a few specific groups of patients in whom an underlying disease confers such increased risk, and in addition in whom the standard treatments may themselves cause dyslipidaemia that may contribute to the risk of ASCVD. These include: (i) chronic immune-mediated inflammatory disease, (ii) patients with human immunodeficiency virus (HIV), and (iii) patients with severe mental illness. The management principles are the same in these patient groups, but their management may need to address specific issues related to individual dyslipidaemias and drug safety. Details are provided in the Supplementary Data document.

\section{Inflammation}

Recent advances in basic science have established a fundamental role for low-degree chronic inflammation in mediating all stages of atherosclerosis, from initiation through progression and, ultimately, to the rupture of plaque and ensuing thrombotic complications of atherosclerosis. The cellular and molecular interactions involved during atherogenesis are fundamentally not different from those in chronic inflammatory-fibroproliferative diseases, such as rheumatoid arthritis (RA), glomerulosclerosis, or pulmonary fibrosis [525]. Almost all cell types of the immuno-inflammatory system, such as macrophages, and $\mathrm{T}$ - and B-cells, as well as many pro- and anti-inflammatory cytokines and chemokines, have been identified during the process of atherosclerosis [526].

Interestingly, cholesterol accumulation in cells triggers the inflammasome response and results in the production of inflammatory mediators such as interleukin (IL)-1 $\beta$. Numerous animal studies, using the knockout model, have demonstrated that inflammation and the immune system both play crucial roles during atherogenesis [527].

During inflammatory processes, large numbers of acute-phase proteins have been identified, and several clinical studies have reported Creactive protein [528] to be the most useful serum marker of inflammation, even though it has poor specificity for any particular inflammation process, including atherosclerosis. The high-sensitivity Creactive protein diagnostic test was developed to detect very low levels of C-reactive protein, and thereby enable a more accurate and precise measure of chronic inflammation compared with standard C-reactive protein [529]. This diagnostic tool differs only in the range of C-reactive protein levels that it can detect. Several studies have found that elevated levels of high-sensitivity C-reactive protein in the blood are associated with an increased risk of $\mathrm{CV}$ events and could be used to predict clinical outcomes independently of cholesterol levels [530,531]. Other studies have not been able to show any relationship between lowgrade chronic inflammation, as indicated by high-sensitivity C-reactive protein levels, and increased risk of CV [532-535]. Finally, genetic studies of large population cohorts have not demonstrated that chronic elevated high-sensitivity C-reactive protein increases the risk of atherosclerotic events [536]. Nevertheless, in some guidelines, high-sensitivity C-reactive protein has been added to traditional risk factors for prognostic information, especially for patients at intermediate risk $[537,538]$.

Statins have been shown to reduce C-reactive protein secretion by hepatocytes [539], and a series of clinical trials and post hoc analyses have found that beneficial outcomes after statin therapy relate both to a reduction in cholesterol levels and reduced inflammation [540-544]. The Justification for the Use of Statins in Prevention: an Intervention Trial Evaluating Rosuvastatin (JUPITER) trial [542] demonstrated that in primary prevention for individuals with chronically elevated C-reactive protein ( $>2 \mathrm{mg} / \mathrm{L}$ ), statin treatment markedly reduced CV events [545]. It is of note that other lipid-lowering agents, such as ezetimibe and more recently the anti-PCSK9 mAbs, do not influence high-sensitivity C-reactive protein levels [546,547], but lead to further significant reductions in CV events when added to statin therapy.

Specific anti-inflammatory treatment was tested in the Canakinumab Antiinflammatory Thrombosis Outcome Study (CANTOS) trial [548]. In patients with previous MI and chronic elevated highsensitivity C-reactive protein levels, all on optimal medical treatment, including statins, the anti-IL- $1 \beta \mathrm{mAb}$ canakinumab dose-dependently reduced high-sensitivity $\mathrm{C}$-reactive protein and significantly lowered the rate of recurrent CV events compared with placebo, independently of the level of lipid lowering. Not surprisingly, there was a slight increase in the risk of severe and fatal infections associated with canakinumab. This study was the first to highlight the positive correlation between high-sensitivity C-reactive protein and CV events, where lower achieved high-sensitivity C-reactive protein values were directly correlated with a lower risk of future CV events [548]. Nevertheless, the FDA declined to approve canakinumab for $\mathrm{CV}$ risk reduction on the strength of data from the CANTOS study. As canakinumab treatment has not been tested against anti-PCSK9 $\mathrm{mAb}$ and/or ezetimibe added to statin therapy, the question of residual risk remains for patients with elevated high-sensitivity C-reactive protein despite achieving very low (below goal) LDL-C values, and whether patients with very low LDL-C would benefit from anti-IL-1 $\beta$ treatment or other anti-inflammatory agents. In addition, all currently recommended lipid-lowering drugs, including anti-PCSK9 mAbs, have demonstrated beneficial effects on atherosclerotic plaque composition as well as plaque volume regression; such results are still missing for anti-inflammatory treatment. Another anti-inflammatory approach using methotrexate was tested in the Cardiovascular Inflammation Reduction Trial (CIRT) [549]. Very low-dose methotrexate (10 mg weekly), a proven anti-inflammatory regimen that reduces tumour necrosis factor (TNF), IL-6, and C-reactive protein levels and is widely used in the treatment of RA, was allocated vs. placebo to 7000 stable CAD patients. This study was stopped prematurely due to futility. Interestingly, this regimen of methotrexate had 
Table 13

Summary of recommendations for monitoring lipids and enzymes in patients, before and on lipid-lowering therapy.

Testing lipids

How often should lipids be tested?

- Before starting lipid-lowering drug treatment, at least two measurements should be made, with an interval of 1-12 weeks, with the exception of conditions where prompt drug treatment is suggested, such as ACS and very high-risk patients.

How often should a patient's lipids be tested after starting lipid-lowering treatment?

- After starting treatment: $8( \pm 4)$ weeks.

- After adjustment of treatment: $8( \pm 4)$ weeks until the goal is achieved.

How often should lipids be tested once a patient has achieved the target or optimal lipid level?

- Annually (unless there are adherence problems or other specific reasons for more frequent reviews).

Monitoring liver and muscle enzymes

How often should liver enzymes (ALT) be routinely measured in patients on lipid-lowering drugs?

- Before treatment.

- Once, 8-12 weeks after starting a drug treatment or after dose increase.

- Routine control of ALT thereafter is not recommended during statin treatment, unless symptoms suggesting liver disease evolve. During treatment with fibrates, control of ALT is still recommended.

What if liver enzymes become elevated in a person taking lipid-lowering drugs? If ALT $<3 \times \mathrm{ULN}$

- Continue therapy.

- Recheck liver enzymes in 4-6 weeks.

If ALT rises to $\geq 3 \times$ ULN

- Stop lipid-lowering therapy or reduce dose and recheck liver enzymes within 4-6 weeks.

- Cautious reintroduction of therapy may be considered after ALT has returned to normal.

- If ALT remains elevated check for the other reasons.

How often should CK be measured in patients taking lipid-lowering drugs? Pre-treatment

o Before starting therapy.

o If baseline CK is $>4 \times \mathrm{ULN}$, do not start drug therapy; recheck.

Monitoring:

o Routine monitoring of CK is not necessary.

o Check CK if patient develops myalgia.

Be alert regarding myopathy and CK elevation in patients at risk, such as: elderly patients, those on concomitant interfering therapy, multiple medications, liver or renal disease, or athletes.

What if CK becomes elevated in a person taking lipid-lowering drugs? Reevaluate indication for statin treatment. If $\geq 4 \times$ ULN:

- If CK > $10 \times$ ULN: stop treatment, check renal function, and monitor CK every 2 weeks.

- If CK $<10 \times$ ULN: if no symptoms, continue lipid-lowering therapy while monitoring CK between 2 and 6 weeks.

- If CK $<10 \times$ ULN: if symptoms present, stop statin and monitor normalization of CK, before rechallenge with a lower statin dose.

- Consider the possibility of transient CK elevation for other reasons such as exertion.

- Consider myopathy if CK remains elevated.

- Consider combination therapy or an alternative drug. O If $<4 \times$ ULN:

- If no muscle symptoms, continue statin (patient should be alerted to report symptoms; check CK).

- If muscle symptoms, monitor symptoms and CK regularly.

- If symptoms persist, stop statin and re-evaluate symptoms after 6 weeks; re-evaluate indication for statin treatment.

- Consider rechallenge with the same or another statin.

- Consider low-dose statin, alternate day or once/twice weekly dosing regimen, or combination therapy.

For details on CK elevation and treatment of muscular symptoms during statin treatment see algorithm in Supplementary Fig. 4. In which patients should HbA1c or blood glucose be checked?

- Regular checks of HbA1c or glucose should be considered in patients at high-risk of developing diabetes, and on high-dose statin treatment.

- Groups to be considered for glucose control are the elderly and patients with metabolic syndrome, obesity, or other signs of insulin resistance.

ACS = acute coronary syndromesyndrome; ALT = alanine aminotransferase; $\mathrm{CK}=$ creatine kinase; ULN = upper limit of normal.

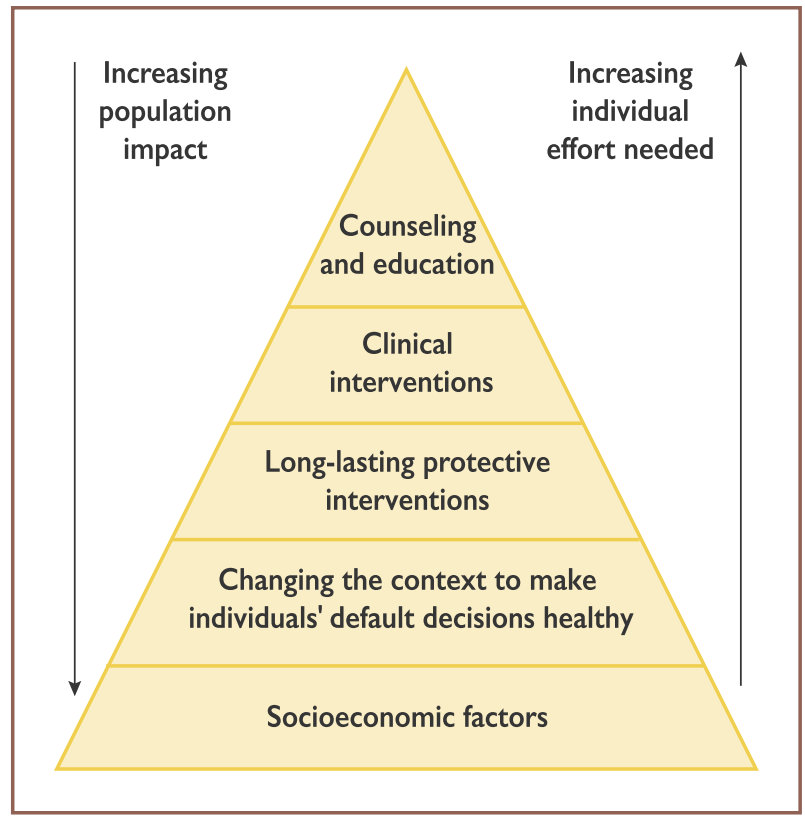

Fig. 5. Health impact pyramid.

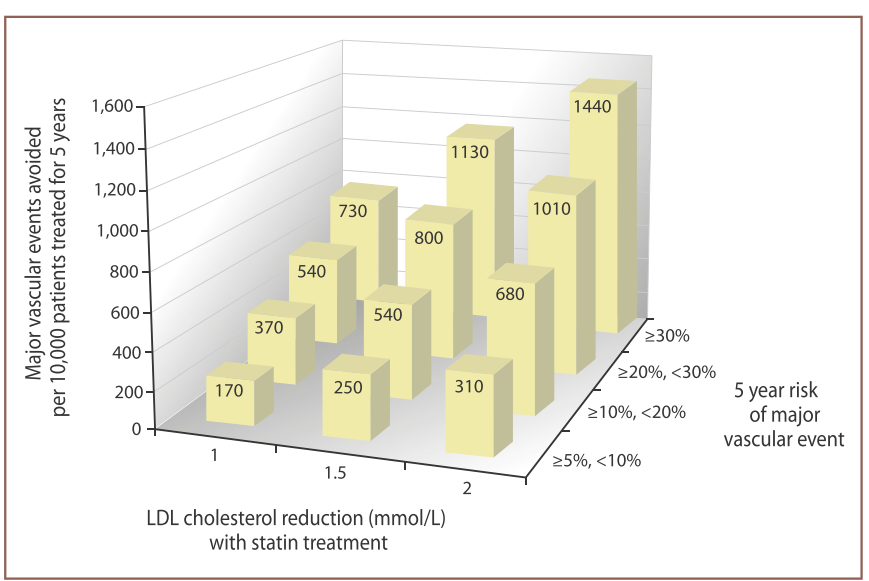

Fig. 6. Absolute reductions in major vascular events with statin therapy [233]. LDL = low-density lipoprotein. Reproduced from The Lancet, 388/10059, Collins et al., 'Interpretation of the evidence for the efficacy and safety of statin therapy', 2532-2561, 2016, with permission from Elsevier.

no effect on either IL-6 or high-sensitivity C-reactive protein blood levels in this population, which could explain the neutral results of this trial [550]. Based on the current level of evidence, no further recommendations on the use of anti-inflammatory agents can be made [551].

\section{Monitoring of lipids and enzymes in patients on lipid-lowering therapy}

Evidence concerning which tests should be carried out to monitor lipids in patients on treatment is limited. Similar limited evidence applies to tests of possible toxicity, such as ALT and CK. Recommendations stem from consensus rather than evidence-based medicine. 
Response to therapy can be assessed at 6-8 weeks from initiation of therapy, but response to lifestyle may take longer. Standard practice for subsequent follow-up monitoring is 6-12 months, but such monitoring intervals are arbitrary. As a minimum, LDL-C should be assessed whenever available, but better management decisions will probably occur if a full lipid profile is performed, including HDL-C and TGs. NonHDL-C or ApoB should also be analysed, and used as a secondary treatment target. A separate issue is the impact of regular lipid monitoring in promoting patient adherence to lifestyle changes or drug regimens that impact positively on their health, as found in a range of studies. It is unclear whether only the process of monitoring is critical in achieving this or whether a combination of education, regular contact, and adherence assessment is required.

Where pharmacological lipid-lowering therapy is implemented, safety blood tests are advised, including ALT and CK at baseline, to identify the limited number of patients where treatment is contraindicated. CK should be checked in patients at high-risk for myopathy, such as the very elderly with comorbidities, patients with antecedents of muscle symptoms, or patients receiving interacting drugs. A mild and typically transient elevation of ALT is seen in about $2 \%$ of patients and normalization is seen with continuing therapy [240,244,552]. Recent reviews are encouraging regarding the safety of long-term statin therapy and statin-induced liver injury is reported to be very uncommon [243,244,553-555]. ALT is recommended before the start of statin therapy; routine control of ALT during treatment is not recommended but should be performed, if indicated, based on clinical observations. During fibrate therapy, regular ALT control is still recommended. In patients whose liver function tests increase to above three times the ULN, explanations such as alcohol ingestion or NAFLD should be sought, and the levels monitored. If levels remain elevated then lipid-lowering therapy should be stopped, but may be cautiously reintroduced under monitoring after levels have returned to normal.

There is no predictive value of routine repeat CK testing for rhabdomyolysis since the level can increase for many reasons, including muscle injury or excess muscular exercise. However, CK must be assessed immediately in patients who present with muscle pain and weakness, and especially in the elderly, and treatment stopped if CK rises to $>10$ times the ULN. Strategies to handle CK elevations are given in Table 13.

Due to the increased frequency of DM during statin treatment [247,249,556,557], regular checks of HbA1c should be considered in patients at high-risk of developing DM and under high-dose statin treatment. Groups to be considered for glucose control are the elderly or those with MetS, obesity, or signs of insulin resistance.

\section{Cost-effectiveness of cardiovascular disease prevention by lipid modification}

In 2015, there were $>85$ million people in Europe living with CVD [558]. Aging populations [559], unhealthy diets, smoking, sedentary lifestyles, increasing obesity, and diabetes [560-563] are the main contributors. CVD cost the European Union about $€ 210$ billion in 2015, one-half of which was in healthcare costs $(\sim 8 \%$ of total healthcare expenditure), and the other half in productivity losses and informal care [558].

In these Guidelines, the Joint Task Force recommends a range of actions to reduce CVD by targeting plasma lipids, ranging from population-wide initiatives to promote healthy lifestyles to individual-level interventions to reduce CVD risk factors, such as unhealthy diets and high lipid levels. Cost-effectiveness analysis can help target resources for interventions where the net health gain is greatest in relation to the net resources, and is increasingly required across Europe [564].
However, cost-effectiveness depends on available resources, the costs of services, and disease risk in the population, and results obtained in one country might not be valid in another [565]. In addition, to fully capture the long-term effects of interventions, cost-effectiveness studies combining evidence from RCTs with modelling and limitations in both could affect the reliability of findings. Here, the evidence for the costeffectiveness of ASCVD preventive interventions with respect to lipid modification is summarized; further scrutiny in view of local circumstances is recommended.

The health impact pyramid summarizes the evidence on the relative efforts and costs in relation to health impact (Fig. 5), with interventions with the broadest impact on populations at the base and interventions requiring considerable individual effort at the top [566]. There is consensus that all the levels of the pyramid should be targeted but that emphasis should be placed on the lower levels. This would address the persistent socio-economic divide in $\mathrm{CV}$ health despite major improvements in ASCVD treatment [558].

More than one-half of the reduction in CV mortality over the last three decades has been attributed to population-level changes in CV risk factors, primarily reductions in plasma cholesterol, BP levels, and smoking [560-563,567]. Lifestyle changes at the population level may be more cost-effective than lifestyle and drug interventions at the individual level, particularly when targeted to populations at increased risk. Awareness and knowledge of how lifestyle risk factors lead to CVD has increased in recent decades. Moreover, legislation promoting a healthy lifestyle, such as reduced salt intake and smoking bans, has been reported to be cost-effective in preventing CVD [568-573], and initiatives to improve infrastructure and promote physical activity have shown promise [574,575]. A number of structural strategies at international, national, and regional levels combined can substantially reduce CVD morbidity and mortality [576,577]. Individual-level interventions to improve diet [578,579], increase physical activity [580], and stop smoking [581] could also be cost-effective [582]. However, suboptimal adherence limits benefits [583,584], and interventions to improve adherence, such as electronic device reminders to reinforce favourable health behaviours, are increasingly being investigated [585].

All statin regimens and ezetimibe are now generically available across Europe. There is strong evidence that lowering blood cholesterol levels using low-cost statins is widely cost-effective [586-590] in many categories of patients. For secondary prevention of CVD, the evidence suggests that statin treatments are highly cost-effective [586,590,591], and adding low-cost ezetimibe to high-intensity statin therapy further reduces LDL-C and CVD risk cost-effectively [592]. In primary ASCVD prevention, the evidence indicates that generic statin-based treatments are cost-effective for people at least down to 1\% annual total CVD risk and could be cost-effective at even lower risk [589], with the highest tolerated statin dose likely the most cost-effective [591,593,594]. Importantly, many patients on statin treatment fail to take their medications adequately and/or to reach their therapeutic goals [595], with clinical and economic consequences [596,597]. Reinforcing measures aimed at improving adherence to treatment is cost-effective [598-600].

Studies have shown that at mid-2018 prices PCSK9 inhibitors were largely not cost-effective. ${ }^{601-604}$ Their cost-effectiveness is improved in selected high-risk patients, such as those with clinical CVD or FH, other comorbidities, and high LDL-C levels. ${ }^{605,606}$ However, at lower prices, PCSK9 inhibitors would become cost-effective in a wider range of highrisk patients; recent price reductions may therefore lead to increased use. $^{607}$ Cost-effectiveness evidence for other lipid-modifying therapies is lacking.

Effective interventions to prevent ASCVD, including statins, typically exhibit similar relative risk reductions across categories of 
Box 8

Key messages

Prevention of CVD by lifestyle changes, medication, or both is cost-effective in many scenarios, including population-based approaches and actions directed at individuals at increased CVD risk.

Cost-effectiveness depends on several factors, including baseline CVD risk and LDL levels, cost of treatment, and uptake of preventive strategies.

Interventions to prevent CVD are more cost-effective among individuals and populations at higher CVD risk.

Cost-effectiveness analyses are importantly informed by assumptions about long-term disease prognosis and treatment effects.

Strengthening of the evidence to inform these assumptions is encouraged.

CVD = cardiovascular disease; LDL = low-density lipoprotein.

Box 9

Gaps in the evidence

Cost-effectiveness requires evidence for effects of interventions on health and healthcare over a long time period; modelling techniques fill gaps. More data are needed from RCTs and observational studies.

Direct evidence of effects of lipid-modifying treatments on overall mortality, particularly among people at low-to-moderate CVD risk, older people, and for newer interventions, is lacking. Long-term post-trial follow-up in RCTs should be encouraged.

The cost-effectiveness of using lifetime CVD risk and more precise CVD risk scores to target interventions needs further investigation.

$\mathrm{CVD}=$ cardiovascular disease RCT $=$ randomized controlled trial.

patients, including by ASCVD risk; therefore, health benefits and costeffectiveness are greater among people at higher ASCVD risk (Fig. 6) $[36,233]$. Consequently, increased efforts and higher-intensity interventions should be aimed at individuals and populations at higher ASCVD risk.

Box 8 lists the key messages regarding the cost-effectiveness of CVD prevention by lipid modification, and Box 9 highlights gaps in the evidence.

\section{Strategies to encourage adoption of healthy lifestyle changes and adherence to lipid-modifying therapies}

Helping patients to change to healthier lifestyle habits is most effectively achieved through formal programmes of preventive care, possibly because of the intensive follow-up and multidisciplinary expertise they provide. ${ }^{608}$ However, in everyday care, adherence to both healthy lifestyle changes and medication regimens is a challenge to patients and professionals.

A comprehensive patient- and family-centred approach located in one healthcare setting is recommended rather than addressing single risk factors with more than one intervention in different locations. Box 10 includes some useful techniques when counselling patients for behavioural change.

A comprehensive approach to improving adherence to medication is described in the Supplementary Data document.

\section{Key messages}

1. Cholesterol and risk. Prospective studies, randomized trials, and Mendelian randomization studies have all shown that raised LDL-C is a cause of ASCVD. Throughout the range of LDL-C levels, 'lower is better' with no lower threshold, at least down to $\sim 1 \mathrm{mmoL} / \mathrm{L}$. Lowering LDL-C may yield worthwhile benefits in patients with average or below average LDL-C who are already receiving LDL-Clowering treatment. The proportional reduction in ASCVD risk achieved by lowering LDL-C (e.g. with a statin, ezetimibe, or PCSK9-inhibitor) depends on the absolute reduction in LDL-C, with each $1 \mathrm{mmoL} / \mathrm{L}$ reduction corresponding to a reduction of about one-fifth in ASCVD.

2. PCSK-9 inhibitors. Large trials have shown that PCSK9 inhibitors further reduce ASCVD risk when given on top of statin-based therapy and their use may need to be restricted to those at the highest risk for ASCVD.

3. Use of cardiac imaging for risk stratification. CAC score

Box 10

Methods for enhancing adherence to lifestyle changes

1. Explore motivation and identify ambivalence. Weigh pros and cons for change, assess and build self-efficacy and confidence, and avoid circular discussion.

2. Offer support, and establish an alliance with the patient and his/her family.

3. Involve the partner, other household members, or caregiver who may be influential in the lifestyle of the patient.

4. Use the OARS method (Open-ended questions, Affirmation, Reflective listening, Summarising when discussing behaviour changes (www. smartrecovery.org/wp-content/uploads/2017/03/UsingMIinSR.pdf).

5. Tailor advice to an individual patient's culture, habits, and situation

6. Use SMART goal setting (negotiate goals for change that are Specific, Measurable, Achievable, Realistic, and Timely). Follow-up on goals and record progress on a shared record. 
assessment with CT may be helpful in reaching decisions about treatment in people who are at moderate risk of ASCVD. Obtaining such a score may assist in discussions about treatment strategies in patients where the LDL-C goal is not achieved with lifestyle intervention alone and there is a question of whether to institute LDL-Clowering treatment. Assessment of arterial (carotid or femoral) plaque burden on ultrasonography may also be informative in these circumstances.

4. Use of ApoB in risk stratification. ApoB may be a better measure of an individual's exposure to atherosclerotic lipoproteins, and hence its use may be particularly helpful for risk assessment in people where measurement of LDL-C underestimates this burden, such as those with high TG, DM, obesity, or very low LDL-C.

5. Use of $L p(a)$ in risk stratification. A one-off measurement of $L p(a)$ may help to identify people with very high inherited Lp(a) levels who may have a substantial lifetime risk of ASCVD. It may also be helpful in further risk stratification of patients at high risk of ASCVD, in patients with a family history of premature CVD, and to determine treatment strategies in people whose estimated risk is on the border of risk categories.

6. Intensification of treatment goals. It is important to ensure that treatment of the highest-risk patients achieves the largest LDL-C reduction possible. These Guidelines aim to support this by setting both a minimum percentage LDL-C reduction (50\%) and an absolute LDL-C treatment goal of $<1.4 \mathrm{mmoL} / \mathrm{L}(<55 \mathrm{mg} / \mathrm{dL})$ for very-high-risk patients, and $<1.8 \mathrm{mmoL} / \mathrm{L}(<70 \mathrm{mg} / \mathrm{dL})$ for highrisk patients. It is recommended that $\mathrm{FH}$ patients with ASCVD or who have another major risk factor are treated as very-high risk, and those with no prior ASCVD or other risk factors as high-risk.

7. Treatment of patients with recent ACS. New randomized trials support a strategy of intensification of LDL-C-lowering therapy in very-high-risk patients with ACS (MI or unstable angina). If the specified LDL-C treatment goal is not achieved after 4-6 weeks with the highest tolerated statin dose and ezetimibe, it is appropriate to add a PCSK9 inhibitor.

8. Safety of low LDL cholesterol concentrations. There are no known adverse effects of very low LDL-C concentrations [e.g. $<1$ $\mathrm{mmoL} / \mathrm{L}(40 \mathrm{mg} / \mathrm{dL})]$.

9. Management of statin 'intolerance'. While statins rarely cause serious muscle damage (myopathy, or rhabdomyolysis in the most severe cases), there is much public concern that statins may commonly cause less serious muscle symptoms. Such statin 'intolerance' is frequently encountered by practitioners and may be difficult to manage. However, placebo-controlled randomized trials have shown very clearly that true statin intolerance is rare, and that it is generally possible to institute some form of statin therapy (e.g. by changing the statin or reducing the dose) in the overwhelming majority of patients at risk of ASCVD.

10. Statin treatment for older people. A meta-analysis of randomized trials has shown that the effects of statin therapy are determined by the absolute reduction in LDL-C as well as the baseline ASCVD risk, and are independent of all known risk factors, including age. Statin therapy in older people should therefore be considered according to the estimated level of risk and baseline LDL-C, albeit with due regard to an individual's underlying health status and the risk of drug interactions. There is less certainty about the effects of statins in individuals aged $>75$ years, particularly in primary prevention. Statin therapy should be started at a low dose if there is significant renal impairment and/or the potential for drug interactions, and then titrated upwards to achieve LDL-C treatment goals.

\section{Gaps in the evidence}

- Prospective studies are needed to investigate the incremental value of reclassifying total CV risk and defining eligibility for lipid-lowering therapy based on CAC scores in individuals at moderate or high-risk.

- Outcome-based comparisons of CAC scores vs. assessment of arterial (carotid or femoral) plaque burden by ultrasonography for CV risk reclassification in people at moderate or high-risk are needed.

- Although calibrated country-specific versions of the SCORE system are available for many European countries, risk charts based on country-specific cohort data are missing for most countries. Regional total event charts (vs. mortality-only charts) are needed.

- Total CV risk estimation by means of the SCORE system and, accordingly, recommendations on eligibility for statins as well as treatment goals are based on TC, whereas LDL-C is the primary lipid analysis method for screening, diagnosis, and management.

- There are no outcome-based comparisons of LDL-C vs. ApoB as primary measurement methods for screening, diagnosis, and management.

- Against a background of genetic and randomized clinical trial evidence showing no significant effect of increasing HDL levels on the risk of CVD events, the clinical impact of therapies altering the function of HDL particles is unknown. More evidence is needed regarding the apparently adverse association of extremely high levels of HDL-C with clinical outcomes.

- Dedicated studies assessing outcomes with specific Lp(a)-lowering therapies are warranted.

- More evidence is needed for PCSK9 inhibitors in specific populations, including patients with severe CKD and on dialysis, patients with HIV infection, in children and adolescents with $\mathrm{FH}$, after heart transplantation, and during pregnancy.

- The effects of PCSK9 inhibition in all body compartments (as with siRNA or antisense) or only within plasma (as with monoclonal antibodies) remain to be established.

- How early should a PCSK9 inhibitor be initiated in patients with ACS or stroke? In view of evidence of sustained clinical benefit associated with the early initiation of statin treatment in the acute phase of ACS or stroke, the optimal timing of PCSK9 inhibitor treatment in ACS and stroke patients remains to be addressed in outcome studies.

- Whether very low LDL-C levels achieved with the combination of statin, ezetimibe, and PCSK9 inhibitor reduce the need for further PCI remains to be addressed in outcome studies.

- In patients with chronic HF, a small benefit of n-3 PUFAs has been shown in one RCT and merits further investigation.

- What is the optimal screening programme for detecting FH?

- In view of limited access to genetic testing in several environments, more evidence is needed regarding outcomes with only clinical vs. genetic screening and diagnosis of FH.

- More RCT evidence is required to support the use of statin-based treatment in older people (aged $\geq 75$ years, but particularly in those aged $\geq 80$ years).

- More RCT evidence is needed for statin treatment in kidney transplant recipients.

- There are no data on the effects of statins, ezetimibe, or fibrates on CV events in dyslipidaemic HIV-infected patients.

- More evidence is needed regarding attainment of recommended LDL goals among very high-risk patients in real-world practice in the era of increasingly prescribed combination therapies for LDL lowering. 


\section{6. 'What to do' and 'what not to do' messages from the guidelines}

\section{Recommendations}

\section{CVD risk estimation}

Total risk estimation using a risk estimation system such as SCORE is recommended for asymptomatic adults aged $>40$ years without evidence of CVD, DMCKD, FH, or LDL $>4.9 \mathrm{mmol} / \mathrm{L}$ (>190 mg/dL).

High- and very-high-risk individuals may be identified on the basis of documented CVD, DM, moderate-to-severe renal disease, very high levels of individual risk factors, $\mathrm{FH}$, or a high SCORE risk, and are a priority for advice and management of all risk factors.

Risk scores developed for the general population are not recommended for $\mathrm{CV}$ risk assessment in patients with $\mathrm{DM}$ or $\mathrm{FH}$. Lipid analyses for CVD risk estimation

TC is to be used for the estimation of total CV risk by means of the SCORE system.

HDL-C analysis is recommended to further refine risk estimation using the online SCORE system.

LDL-C analysis is recommended as the primary lipid analysis method for screening, diagnosis, and management.

TG analysis is recommended as a part of the routine lipid analysis approach.

Non-HDL-C evaluation is recommended for risk assessment, particularly in people with high TG levels, DM, obesity, or very low LDL-C levels.

ApoB analysis is recommended for risk assessment, particularly in people with high TG levels, DM, obesity, or MetS, or very low LDL-C levels. It can be used as an alternative to LDL-C, if available, as the primary measurement for screening, diagnosis, and management, and may be preferred over non-HDL-C in people with high TG levels, DM, obesity, or very low LDL-C levels.

\section{Treatment goals for LDL-C}

In secondary prevention for patients at very-high risk, an LDL-C reduction of $\geq 50 \%$ from baseline and an LDL-C goal of $<1.4$ $\mathrm{mmol} / \mathrm{L}(<55 \mathrm{mg} / \mathrm{dL})$ are recommended.

In primary prevention for individuals at very-high risk, an LDL-C reduction of $\geq 50 \%$ from baseline and an LDL-C goal of $<1.4$ $\mathrm{mmol} / \mathrm{L}(<55 \mathrm{mg} / \mathrm{dL})$ are recommended.

In patients at high risk, an LDL-C reduction of $\geq 50 \%$ from baseline and an LDL-C goal of $<1.8 \mathrm{mmol} / \mathrm{L}(<70 \mathrm{mg} / \mathrm{dL})$ are recommended.

\section{Pharmacological LDL-C lowering}

It is recommended that a high-intensity statin is prescribed up to the highest tolerated dose to reach the goals set for the specific level of risk.

If the goals are not achieved with the maximum tolerated dose of a statin, combination with ezetimibe is recommended.

For secondary prevention in patients at very-high risk not achieving their goal on a maximum tolerated dose of a statin and ezetimibe, a combination with a PCSK9 inhibitor is recommended.

For very-high-risk FH patients (that is, with ASCVD or with another major risk factor) who do not achieve their goal on a maximum tolerated dose of a statin and ezetimibe, a combination with a PCSK9 inhibitor is recommended.

\section{Drug treatment of patients with HTG}

Statin treatment is recommended as the first drug of choice for reducing CVD risk in high-risk individuals with HTG [TGs $>2.3$ $\mathrm{mmol} / \mathrm{L}(>200 \mathrm{mg} / \mathrm{dL})]$.

\section{Management of patients with $\mathrm{HeFH}$}

It is recommended that a diagnosis of $\mathrm{FH}$ is considered in patients with $\mathrm{CHD}$ aged $<55$ years for men and $<60$ years for women, in people with relatives with premature fatal or non-fatal CVD, in people with relatives having tendon xanthomas, in people with severely elevated LDL-C levels [in adults $>5 \mathrm{mmol} / \mathrm{L}(>190 \mathrm{mg} / \mathrm{dL})$, in children $>4 \mathrm{mmol} / \mathrm{L}(>150 \mathrm{mg} / \mathrm{dL})$ ], and in first-degree relatives of $\mathrm{FH}$ patients.

It is recommended that $\mathrm{FH}$ is diagnosed using clinical criteria and confirmed, when possible, with DNA analysis.

Once the index case is diagnosed, family cascade screening is recommended.

It is recommended that FH patients with ASCVD or who have another major risk factor are treated as very-high-risk, and those with no prior ASCVD or other risk factors as high-risk.

\begin{tabular}{|l|l|}
\hline I \\
\hline
\end{tabular}


For $\mathrm{FH}$ patients with ASCVD who are at very-high risk, treatment to achieve a $\geq 50 \%$ reduction from baseline and an LDL-C $<1.4 \mathrm{mmol} / \mathrm{L}(<55 \mathrm{mg} / \mathrm{dL})$ is recommended. If goals cannot be achieved, a drug combination is recommended.

Treatment with a PCSK9 inhibitor is recommended in very-high risk FH patients if the treatment goal is not achieved on a maximal tolerated statin plus ezetimibe.

In children, testing for $\mathrm{FH}$ is recommended from the age of 5 years, or earlier if $\mathrm{HoFH}$ is suspected.

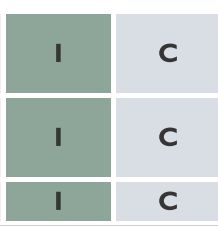

\section{Treatment of dyslipidaemias in older people}

Treatment with statins is recommended for older people with ASCVD in the same way as for younger patients.

Treatment with statins is recommended for primary prevention, according to the level of risk, in older people aged $\leq 75$ years. It is recommended that the statin is started at a low dose if there is significant renal impairment and/or the potential for drug interactions, and then titrated upwards to achieve LDL-C treatment goals.

\section{Treatment of dyslipidaemias in DM}

In patients with T2DM at very-high risk, ${ }^{c}$ an LDL-C reduction of $\geq 50 \%$ from baseline and an LDL-C goal of $<1.4 \mathrm{mmol} / \mathrm{L}$ $(<55 \mathrm{mg} / \mathrm{dL})$ is recommended.

In patients with T2DM at high risk, ${ }^{c}$ an LDL-C reduction of $\geq 50 \%$ from baseline and an LDL-C goal of $<1.8 \mathrm{mmol} / \mathrm{L}(<70 \mathrm{mg} / \mathrm{dL})$ is recommended.

Statins are recommended in patients with T1DM who are at high or very-high risk. ${ }^{c}$

Statin therapy is not recommended in pre-menopausal patients with or without DM who are considering pregnancy, or not using adequate contraception.

\section{Management of patients with ACS}

In all ACS patients without any contraindication or definite history of intolerance, it is recommended that high-dose statin therapy is initiated or continued as early as possible, regardless of initial LDL-C values.

If the LDL-C goal is not achieved after $4-6$ weeks with the maximally tolerated statin dose, combination with ezetimibe is recommended.

If the LDL-C goal is not achieved after 4-6 weeks despite maximal tolerated statin therapy and ezetimibe, adding a PCSK9 inhibitor is recommended.

\begin{tabular}{|l|l|}
\hline I & A \\
\hline I & A \\
\hline I & C \\
\hline
\end{tabular}

\section{Lipid-lowering therapy for prevention of ASCVD events in patients with prior ischaemic stroke}

Patients with a history of ischaemic stroke or TIA are at very-high risk of ASCVD, particularly recurrent ischaemic stroke, so it is recommended that they receive intensive LDL-C-lowering therapy.

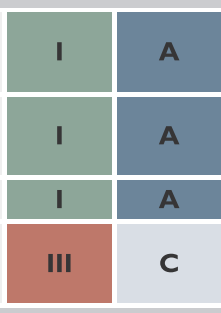

\section{Treatment of dyslipidaemias in chronic HF or valvular heart diseases}

Initiation of lipid-lowering therapy is not recommended in patients with HF in the absence of other indications for their use. Initiation of lipid-lowering treatment is not recommended in patients with aortic valvular stenosis without CAD to slow progression of aortic valve stenosis in the absence of other indications for their use.

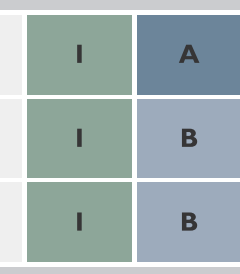

Lipid management in patients with moderate-to-severe (Kidney Disease Outcomes Quality Initiative stages 3-5) CKD

It is recommended that patients with stage 3-5 CKD are considered to be at high or very-high risk of ASCVD.

The use of statins or statin/ezetimibe combination is recommended in patients with non-dialysis-dependent stage 3-5 CKD.

In patients with dialysis-dependent CKD who are free of ASCVD, commencement of statin therapy is not recommended.

\section{Lipid-lowering drugs in patients with PAD (including carotid artery disease)}

In patients with PAD, lipid-lowering therapy-including a maximum tolerated dose of a statin, plus ezetimibe, or a combination with a PCSK9 inhibitor if needed - is recommended to reduce the risk of ASCVD events.

\section{Lipid-lowering drugs in patients with CIID}

The use of lipid-lowering drugs only on the basis of the presence of CIID is not recommended.

\section{Lipid-lowering drugs in patients with SMI}

It is recommended that SMls are used as modifiers for estimating total ASCVD risk.

It is recommended that the same guidelines for the management of total ASCVD risk are used in patients with SMI as are used in patients without such disease.

It is recommended that in patients with SMI, intensified attention is paid to adherence to lifestyle changes and to compliance with drug treatment.

$\mathrm{ACS}=$ acute coronary syndrome(s); Apo = apolipoprotein; ASCVD = atherosclerotic cardiovascular disease; $C A D=$ coronary artery disease; $C H D=$ coronary heart disease; $\mathrm{CIID}=$ chronic immune-mediated inflammatory diseases; $\mathrm{CKD}=$ chronic kidney disease; $\mathrm{CV}=$ cardiovascular; $\mathrm{CVD}=$ cardiovascular disease; $\mathrm{DM}=$ diabetes mellitus; $\mathrm{FH}=$ fam ilial hypercholesterolaemia; HDL-C = high-density lipoprotein cholesterol; HeFH = heterozygous FH; HF = heart failure; HoFH = homozygous FH; HTG = hypertriglyceridaemia; LDL-C = low-density lipoprotein cholesterol; MetS = metabolic syndrome; PAD = peripheral arterial disease; PCSK9 = proprotein convertase subtilisin/kexin type 9; SCORE = Systematic Coronary Risk Estimation; SMI = severe mental illness; TC = total cholesterol; TG = triglycerides; TIA = transient ischaemic event; T1DM = type $1 \mathrm{DM}$; T2DM = type 2 DM.

${ }^{a}$ Class of recommendation.

' Level of evidence. 


\section{ESC entities having participated in the development of this document}

Associations: Acute Cardiovascular Care Association (ACCA), Association of Cardiovascular Nursing \& Allied Professions (ACNAP), European Association of Cardiovascular Imaging (EACVI), European Association of Preventive Cardiology (EAPC), European Association of Percutaneous Cardiovascular Interventions (EAPCI).

Councils: Council for Cardiology Practice, Council on Hypertension, Council on Stroke.

Working Groups: Aorta and Peripheral Vascular Diseases, Atherosclerosis and Vascular Biology, Cardiovascular Pharmacotherapy, e-Cardiology, Thrombosis.

The content of these European Society of Cardiology (ESC) Guidelines has been published for personal and educational use only. No commercial use is authorized. No part of the ESC Guidelines may be translated or reproduced in any form without written permission from the ESC. Permission can be obtained upon submission of a written request to Oxford University Press, the publisher of the European Heart Journal and the party authorized to handle such permissions on behalf of the ESC (journals.permissions@oxfordjournals.org).

Disclaimer. The ESC/EAS Guidelines represent the views of the ESC and EAS, and were produced after careful consideration of the scientific and medical knowledge, and the evidence available at the time of their publication. The ESC and EAS is not responsible in the event of any contradiction, discrepancy, and/or ambiguity between the ESC/EAS Guidelines and any other official recommendations or guidelines issued by the relevant public health authorities, in particular in relation to good use of healthcare or therapeutic strategies. Health professionals are encouraged to take the ESC/EAS Guidelines fully into account when exercising their clinical judgment, as well as in the determination and the implementation of preventive, diagnostic, or therapeutic medical strategies; however, the ESC/EAS Guidelines do not override, in any way whatsoever, the individual responsibility of health professionals to make appropriate and accurate decisions in consideration of each patient's health condition and in consultation with that patient and, where appropriate and/or necessary, the patient's caregiver. Nor do the ESC/ EAS Guidelines exempt health professionals from taking into full and careful consideration the relevant official updated recommendations or guidelines issued by the competent public health authorities, in order to manage each patient's case in light of the scientifically accepted data pursuant to their respective ethical and professional obligations. It is also the health professional's responsibility to verify the applicable rules and regulations relating to drugs and medical devices at the time of prescription.

\section{Appendix A. Supplementary data}

Supplementary Data with additional Supplementary Tables, Boxes, and text complementing the full text-as well as sections on other features of a healthy diet contributing to cardiovascular disease prevention, chronic immune-mediated inflammatory diseases, HIV patients, severe mental illness, and adhering to medications along with the related references-are available on the European Heart Journal website and via the ESC website at www.escardio.org/guidelines.

Supplementary data to this article can be found online at https:// doi.org/10.1016/j.atherosclerosis.2019.08.014.

\section{References}

[1] A.L. Catapano, I. Graham, G. De Backer, O. Wiklund, M.J. Chapman, H. Drexel, A.W. Hoes, C.S. Jennings, U. Landmesser, T.R. Pedersen, Z. Reiner, G. Riccardi, M.R. Taskinen, L. Tokgozoglu, W.M.M. Verschuren, C. Vlachopoulos, D.A. Wood, J.L. Zamorano, M.T. Cooney, ESC Scientific Document Group, ESC/EAS Guidelines for the management of dyslipidaemias, Eur. Heart J. 37 (2016) 2999-3058 2016.

[2] B.A. Ference, H.N. Ginsberg, I. Graham, K.K. Ray, C.J. Packard, E. Bruckert, R.A. Hegele, R.M. Krauss, F.J. Raal, H. Schunkert, G.F. Watts, J. Boren, S. Fazio,
J.D. Horton, L. Masana, S.J. Nicholls, B.G. Nordestgaard, B. van de Sluis, M.R. Taskinen, L. Tokgozoglu, U. Landmesser, U. Laufs, O. Wiklund, J.K. Stock, M.J. Chapman, A.L. Catapano, Low-density lipoproteins cause atherosclerotic cardiovascular disease. 1. Evidence from genetic, epidemiologic, and clinical studies. A consensus statement from the European Atherosclerosis Society Consensus Panel, Eur. Heart J. 38 (2017) 2459-2472.

[3] N. Townsend, M. Nichols, P. Scarborough, M. Rayner, Cardiovascular disease in Europe-epidemiological update 2015, Eur. Heart J. 36 (2015) 2696-2705.

[4] M.T. Cooney, A. Dudina, P. Whincup, S. Capewell, A. Menotti, P. Jousilahti, I. Njolstad, R. Oganov, T. Thomsen, A. Tverdal, H. Wedel, L. Wilhelmsen, I. Graham, S.C.O.R.E. Investigators, Re-evaluating the Rose approach: comparative benefits of the population and high-risk preventive strategies, Eur. J. Cardiovasc. Prev. Rehabil. 16 (2009) 541-549.

[5] World Health Organization, Global status report on noncommunicable diseases 2014, World Health Organization (2014) Geneva, Switzerland https://www.who. int/nmh/publications/ncd-status-report-2014/en/, Accessed date: 17 July 2019.

[6] M.T. Cooney, A.L. Dudina, I.M. Graham, Value and limitations of existing scores for the assessment of cardiovascular risk: a review for clinicians, J. Am. Coll. Cardiol. 54 (2009) 1209-1227.

[7] K. Hajifathalian, P. Ueda, Y. Lu, M. Woodward, A. Ahmadvand, C.A. Aguilar Salinas, F. Azizi, R. Cifkova, M. Di Cesare, L. Eriksen, F. Farzadfar, N. Ikeda, D. Khalili, Y.H. Khang, V. Lanska, L. Leon-Munoz, D. Magliano, K.P. Msyamboza, K. Oh, F. Rodriguez-Artalejo, R. Rojas-Martinez, J.E. Shaw, G.A. Stevens, J. Tolstrup, B. Zhou, J.A. Salomon, M. Ezzati, G. Danaei, A novel risk score to predict cardiovascular disease risk in national populations (Globorisk): a pooled analysis of prospective cohorts and health examination surveys, Lancet Diabetes Endocrinol 3 (2015) 339-355.

[8] M.T. Cooney, A. Dudina, R. D'Agostino, I.M. Graham, Cardiovascular risk-estimation systems in primary prevention: do they differ? Do they make a difference? Can we see the future? Circulation 122 (2010) 300-310.

[9] J. Perk, G. De Backer, H. Gohlke, I. Graham, Z. Reiner, M. Verschuren, C. Albus, P. Benlian, G. Boysen, R. Cifkova, C. Deaton, S. Ebrahim, M. Fisher, G. Germano, R. Hobbs, A. Hoes, S. Karadeniz, A. Mezzani, E. Prescott, L. Ryden, M. Scherer, M. Syvanne, W.J. Scholte op Reimer, C. Vrints, D. Wood, J.L. Zamorano, F. Zannad, European association for cardiovascular prevention \& rehabilitation; ESC Committee for practice guidelines (CPG). European guidelines on cardiovascular disease prevention in clinical practice (version 2012). The fifth Joint task force of the European society of Cardiology and other societies on cardiovascular disease prevention in clinical practice (constituted by representatives of nine societies and by invited experts), Eur. Heart J. 33 (2012) 1635-1701.

[10] M.F. Piepoli, A.W. Hoes, S. Agewall, C. Albus, C. Brotons, A.L. Catapano, M.T. Cooney, U. Corra, B. Cosyns, C. Deaton, I. Graham, M.S. Hall, F.D. Hobbs, M.L. Lochen, H. Lollgen, P. Marques-Vidal, J. Perk, E. Prescott, J. Redon, D.J. Richter, N. Sattar, Y. Smulders, M. Tiberi, H.B. van der Worp, I. van Dis, W.M. Verschuren, M. Authors/Task Force, European guidelines on cardiovascular disease prevention in clinical practice: the sixth Joint task force of the European society of Cardiology and other societies on cardiovascular disease prevention in clinical practice (constituted by representatives of 10 societies and by invited experts)Developed with the special contribution of the European association for cardiovascular prevention \& rehabilitation (EACPR), Eur. Heart J. 37 (2016) 2315-2381 2016 .

[11] M.T. Cooney, R. Selmer, A. Lindman, A. Tverdal, A. Menotti, T. Thomsen, G. DeBacker, D. De Bacquer, G.S. Tell, I. Njolstad, I.M. Graham, SCORE and CONOR investigators, Cardiovascular risk estimation in older persons: SCORE O.P, Eur J Prev Cardiol 23 (2016) 1093-1103.

[12] J.D. Berry, A. Dyer, X. Cai, D.B. Garside, H. Ning, A. Thomas, P. Greenland, L. Van Horn, R.P. Tracy, D.M. Lloyd-Jones, Lifetime risks of cardiovascular disease, N. Engl. J. Med. 366 (2012) 321-329.

[13] H.M.E. Foster, C.A. Celis-Morales, B.I. Nicholl, F. Petermann-Rocha, J.P. Pell, J.M.R. Gill, C.A. O'Donnell, F.S. Mair, The effect of socioeconomic deprivation on the association between an extended measurement of unhealthy lifestyle factors and health outcomes: a prospective analysis of the UK Biobank cohort, Lancet Public Health 3 (2018) e576-e585.

[14] M. Kavousi, S. Elias-Smale, J.H. Rutten, M.J. Leening, R. Vliegenthart, G.C. Verwoert, G.P. Krestin, M. Oudkerk, M.P. de Maat, F.W. Leebeek, F.U. Mattace-Raso, J. Lindemans, A. Hofman, E.W. Steyerberg, A. van der Lugt, A.H. van den Meiracker, J.C. Witteman, Evaluation of newer risk markers for coronary heart disease risk classification: a cohort study, Ann. Intern. Med. 156 (2012) 438-444.

[15] C. Vlachopoulos, P. Xaplanteris, V. Aboyans, M. Brodmann, R. Cifkova, F. Cosentino, M. De Carlo, A. Gallino, U. Landmesser, S. Laurent, J. Lekakis, D.P. Mikhailidis, K.K. Naka, A.D. Protogerou, D. Rizzoni, A. Schmidt-Trucksass, L. Van Bortel, T. Weber, A. Yamashina, R. Zimlichman, P. Boutouyrie, J. Cockcroft, M. O'Rourke, J.B. Park, G. Schillaci, H. Sillesen, R.R. Townsend, The role of vascular biomarkers for primary and secondary prevention. A position paper from the European Society of Cardiology Working Group on peripheral circulation: en dorsed by the Association for Research into Arterial Structure and Physiology (ARTERY) Society, Atherosclerosis 241 (2015) 507-532.

[16] J. Yeboah, R.L. Mcclelland, T.S. Polonsky, G.L. Burke, C.T. Sibley, D. O'Leary, J.J. Carr, D.C. Goff, P. Greenland, D.M. Herrington, Comparison of novel risk markers for improvement in cardiovascular risk assessment in intermediate-risk individuals, J. Am. Med. Assoc. 308 (2012) 788-795.

[17] C.M. Madsen, A. Varbo, B.G. Nordestgaard, Extreme high high-density lipoprotein cholesterol is paradoxically associated with high mortality in men and women: two prospective cohort studies, Eur. Heart J. 38 (2017) 2478-2486.

[18] M.B. Mortensen, E. Falk, D. Li, K. Nasir, M.J. Blaha, V. Sandfort, C.J. Rodriguez, 
P. Ouyang, M. Budoff, Statin trials, cardiovascular events, and coronary artery calcification: implications for a trial-based approach to statin therapy in MESA, JACC Cardiovasc Imaging 11 (2018) 221-230.

[19] J.S. Lin, C.V. Evans, E. Johnson, N. Redmond, E.L. Coppola, N. Smith, Nontraditional risk factors in cardiovascular disease risk assessment: updated evidence report and systematic review for the US Preventive Services Task Force, J. Am. Med. Assoc. 320 (2018) 281-297.

[20] U. Baber, R. Mehran, S. Sartori, M.M. Schoos, H. Sillesen, P. Muntendam, M.J. Garcia, J. Gregson, S. Pocock, E. Falk, V. Fuster, Prevalence, impact, and predictive value of detecting subclinical coronary and carotid atherosclerosis in asymptomatic adults: the BioImage study, J. Am. Coll. Cardiol. 65 (2015) 1065-1074.

[21] M.M. McDermott, C.M. Kramer, L. Tian, J. Carr, J.M. Guralnik, T. Polonsky, T. Carroll, M. Kibbe, M.H. Criqui, L. Ferrucci, L. Zhao, D.S. Hippe, J. Wilkins, D. Xu, Y. Liao, W. McCarthy, C. Yuan, Plaque composition in the proximal superficial femoral artery and peripheral artery disease events, JACC Cardiovasc Imaging 10 (2017) 1003-1012.

[22] H. Sillesen, S. Sartori, B. Sandholt, U. Baber, R. Mehran, V. Fuster, Carotid plaque thickness and carotid plaque burden predict future cardiovascular events in asymptomatic adult Americans, Eur Heart J Cardiovasc Imaging 19 (2018) 1042-1050.

[23] P. Perrone-Filardi, S. Achenbach, S. Mohlenkamp, Z. Reiner, G. Sambuceti, J.D. Schuijf, E. Van der Wall, P.A. Kaufmann, J. Knuuti, S. Schroeder, M.J. Zellweger, Cardiac computed tomography and myocardial perfusion scintigraphy for risk stratification in asymptomatic individuals without known cardiovascular disease: a position statement of the Working Group on Nuclear Cardiology and Cardiac CT of the European Society of Cardiology, Eur. Heart J. 32 (2011) 1986-1993 1993a, 1993b.

[24] H.M. Den Ruijter, S.A. Peters, T.J. Anderson, A.R. Britton, J.M. Dekker, M.J. Eijkemans, G. Engstrom, G.W. Evans, J. de Graaf, D.E. Grobbee, B. Hedblad, A. Hofman, S. Holewijn, A. Ikeda, M. Kavousi, K. Kitagawa, A. Kitamura, H. Koffijberg, E.M. Lonn, M.W. Lorenz, E.B. Mathiesen, G. Nijpels, S. Okazaki, D.H. O'Leary, J.F. Polak, J.F. Price, C. Robertson, C.M. Rembold, M. Rosvall, T. Rundek, J.T. Salonen, M. Sitzer, C.D. Stehouwer, J.C. Witteman, K.G. Moons, M.L. Bots, Common carotid intima-media thickness measurements in cardiovascular risk prediction: a meta-analysis, J. Am. Med. Assoc. 308 (2012) 796-803.

[25] M.W. Lorenz, C. Schaefer, H. Steinmetz, M. Sitzer, Is carotid intima media thickness useful for individual prediction of cardiovascular risk? Ten-year results from the Carotid Atherosclerosis Progression Study (CAPS), Eur. Heart J. 31 (2010) 2041-2048.

[26] P.K. Garg, N.W. Jorgensen, R.L. McClelland, J.A. Leigh, P. Greenland, M.J. Blaha, A.J. Yoon, N.D. Wong, J. Yeboah, M.J. Budoff, Use of coronary artery calcium testing to improve coronary heart disease risk assessment in a lung cancer screening population: the Multi-Ethnic Study of Atherosclerosis (MESA), J Cardiovasc Comput Tomogr 12 (2018) 493-499.

[27] J.C. Hong, R. Blankstein, L.J. Shaw, W.V. Padula, A. Arrieta, J.A. Fialkow, R.S. Blumenthal, M.J. Blaha, H.M. Krumholz, K. Nasir, Implications of coronary artery calcium testing for treatment decisions among statin candidates according to the ACC/AHA cholesterol management guidelines: a cost-effectiveness analysis, JACC Cardiovasc Imaging 10 (2017) 938-952.

[28] I. Cho, S.J. Al'Aref, A.B.O.H. Berger, H. Gransar, V. Valenti, F.Y. Lin, S. Achenbach, D.S. Berman, M.J. Budoff, T.Q. Callister, M.H. Al-Mallah, F. Cademartiri, K. Chinnaiyan, B.J.W. Chow, A. DeLago, T.C. Villines, M. Hadamitzky, J. Hausleiter, J. Leipsic, L.J. Shaw, P.A. Kaufmann, G. Feuchtner, Y.J. Kim, E. Maffei, G. Raff, G. Pontone, D. Andreini, H. Marques, R. Rubinshtein, H.J. Chang, J.K. Min, Prognostic value of coronary computed tomographic angiography findings in asymptomatic individuals: a 6-year follow-up from the prospective multicentre international CONFIRM study, Eur. Heart J. 39 (2018) 934-941.

[29] M. Kavousi, C.S. Desai, C. Ayers, R.S. Blumenthal, M.J. Budoff, A.A. Mahabadi, M.A. Ikram, A. van der Lugt, A. Hofman, R. Erbel, A. Khera, M.H. Geisel, K.H. Jockel, N. Lehmann, U. Hoffmann, C.J. O'Donnell, J.M. Massaro, K. Liu, S. Mohlenkamp, H. Ning, O.H. Franco, P. Greenland, Prevalence and prognostic implications of coronary artery calcification in low-risk women: a meta-analysis, J. Am. Med. Assoc. 316 (2016) 2126-2134.

[31] S.M. Boekholdt, G.K. Hovingh, S. Mora, B.J. Arsenault, P. Amarenco, T.R. Pedersen, J.C. LaRosa, D.D. Waters, D.A. DeMicco, R.J. Simes, A.C. Keech, D. Colquhoun, G.A. Hitman, D.J. Betteridge, M.B. Clearfield, J.R. Downs, H.M. Colhoun, A.M. Gotto Jr., P.M. Ridker, S.M. Grundy, J.J. Kastelein, Very low levels of atherogenic lipoproteins and the risk for cardiovascular events: a metaanalysis of statin trials, J. Am. Coll. Cardiol. 64 (2014) 485-494.

[32] J.J. Brugts, T. Yetgin, S.E. Hoeks, A.M. Gotto, J. Shepherd, R.G. Westendorp, A.J. de Craen, R.H. Knopp, H. Nakamura, P. Ridker, R. van Domburg, J.W. Deckers, The benefits of statins in people without established cardiovascular disease but with cardiovascular risk factors: meta-analysis of randomised controlled trials, BMJ 338 (2009) b2376.

[33] C.P. Cannon, M.A. Blazing, R.P. Giugliano, A. McCagg, J.A. White, P. Theroux, H. Darius, B.S. Lewis, T.O. Ophuis, J.W. Jukema, G.M. De Ferrari, W. Ruzyllo, P. De Lucca, K. Im, E.A. Bohula, C. Reist, S.D. Wiviott, A.M. Tershakovec, T.A. Musliner, E. Braunwald, R.M. Califf, IMPROVE-IT Investigators, Ezetimibe added to statin therapy after acute coronary syndromes, N. Engl. J. Med. 372 (2015) 2387-2397.

[34] Cholesterol Treatment Trialists Collaboration, C. Baigent, L. Blackwell, J. Emberson, L.E. Holland, C. Reith, N. Bhala, R. Peto, E.H. Barnes, A. Keech, J. Simes, R. Collins, Efficacy and safety of more intensive lowering of LDL cholesterol: a meta-analysis of data from 170,000 participants in 26 randomised trials,
Lancet 376 (2010) 1670-1681.

[35] Cholesterol Treatment Trialists Collaboration, J. Fulcher, R. O'Connell, M. Voysey, J. Emberson, L. Blackwell, B. Mihaylova, J. Simes, R. Collins, A. Kirby, H. Colhoun, E. Braunwald, J. La Rosa, T.R. Pedersen, A. Tonkin, B. Davis, P. Sleight, M.G. Franzosi, C. Baigent, A. Keech, Efficacy and safety of LDL-lowering therapy among men and women: meta-analysis of individual data from 174,000 participants in 27 randomised trials, Lancet 385 (2015) 1397-1405.

[36] Cholesterol Treatment Trialists Collaboration, B. Mihaylova, J. Emberson, L. Blackwell, A. Keech, J. Simes, E.H. Barnes, M. Voysey, A. Gray, R. Collins, C. Baigent, The effects of lowering LDL cholesterol with statin therapy in people at low risk of vascular disease: meta-analysis of individual data from 27 randomised trials, Lancet 380 (2012) 581-590.

[37] R.A. Hegele, H.N. Ginsberg, M.J. Chapman, B.G. Nordestgaard, J.A. Kuivenhoven, M. Averna, J. Boren, E. Bruckert, A.L. Catapano, O.S. Descamps, G.K. Hovingh, S.E. Humphries, P.T. Kovanen, L. Masana, P. Pajukanta, K.G. Parhofer, F.J. Raal, K.K. Ray, R.D. Santos, A.F. Stalenhoef, E. Stroes, M.R. Taskinen, A. TybjaergHansen, G.F. Watts, O. Wiklund, European Atherosclerosis Society Consensus Panel. The polygenic nature of hypertriglyceridaemia: implications for definition, diagnosis, and management, Lancet Diabetes Endocrinol 2 (2014) 655-666.

[38] E.J. Mills, B. Rachlis, P. Wu, P.J. Devereaux, P. Arora, D. Perri, Primary prevention of cardiovascular mortality and events with statin treatments: a network metaanalysis involving more than 65,000 patients, J. Am. Coll. Cardiol. 52 (2008) $1769-1781$.

[39] T.R. Pedersen, O. Faergeman, J.J. Kastelein, A.G. Olsson, M.J. Tikkanen, I. Holme, M.L. Larsen, F.S. Bendiksen, C. Lindahl, M. Szarek, J. Tsai, Incremental Decrease in End Points through Aggressive Lipid Lowering (IDEAL) Study Group. High-dose atorvastatin vs usual-dose simvastatin for secondary prevention after myocardial infarction: the IDEAL study: a randomized controlled trial, J. Am. Med. Assoc. 294 (2005) 2437-2445.

[40] N.J. Stone, J.G. Robinson, A.H. Lichtenstein, C.N. Bairey Merz, C.B. Blum, R.H. Eckel, A.C. Goldberg, D. Gordon, D. Levy, D.M. Lloyd-Jones, P. McBride, J.S. Schwartz, S.T. Shero, S.C. Smith Jr., K. Watson, P.W. Wilson, K.M. Eddleman, N.M. Jarrett, K. LaBresh, L. Nevo, J. Wnek, J.L. Anderson, J.L. Halperin, N.M. Albert, B. Bozkurt, R.G. Brindis, L.H. Curtis, D. DeMets, J.S. Hochman, R.J. Kovacs, E.M. Ohman, S.J. Pressler, F.W. Sellke, W.K. Shen, S.C. Smith Jr., G.F. Tomaselli, American College of Cardiology/American Heart Association Task Force on Practice Guidelines, ACC/AHA guideline on the treatment of blood cholesterol to reduce atherosclerotic cardiovascular risk in adults: a report of the American College of Cardiology/American Heart Association Task Force on Practice Guidelines, Circulation 129 (2013) S1-S45 2014.

[41] A.J. Vallejo-Vaz, M. Robertson, A.L. Catapano, G.F. Watts, J.J. Kastelein, C.J. Packard, I. Ford, K.K. Ray, Low-density lipoprotein cholesterol lowering for the primary prevention of cardiovascular disease among men with primary elevations of low-density lipoprotein cholesterol levels of $190 \mathrm{mg} / \mathrm{dL}$ or above: analyses from the WOSCOPS (West of Scotland Coronary Prevention Study) 5-year randomized trial and 20-year observational follow-up, Circulation 136 (2017) 1878-1891.

[42] I. Tabas, K.J. Williams, J. Boren, Subendothelial lipoprotein retention as the initiating process in atherosclerosis: update and therapeutic implications, Circulation 116 (2007) 1832-1844.

[43] J. Boren, K.J. Williams, The central role of arterial retention of cholesterol-rich apolipoprotein-B-containing lipoproteins in the pathogenesis of atherosclerosis: a triumph of simplicity, Curr. Opin. Lipidol. 27 (2016) 473-483.

[44] B.A. Ference, I. Graham, L. Tokgozoglu, A.L. Catapano, Impact of lipids on cardiovascular health: JACC health promotion series, J. Am. Coll. Cardiol. 72 (2018) 1141-1156.

[45] Emerging Risk Factors Collaboration, E. Di Angelantonio, P. Gao, L. Pennells, S. Kaptoge, M. Caslake, A. Thompson, A.S. Butterworth, N. Sarwar, D. Wormser, D. Saleheen, C.M. Ballantyne, B.M. Psaty, J. Sundstrom, P.M. Ridker, D. Nagel, R.F. Gillum, I. Ford, P. Ducimetiere, S. Kiechl, W. Koenig, R.P. Dullaart, G. Assmann, R.B. D'Agostino Sr., G.R. Dagenais, J.A. Cooper, D. Kromhout, A. Onat, R.W. Tipping, A. Gomez-de-la-Camara, A. Rosengren, S.E. Sutherland, J. Gallacher, F.G. Fowkes, E. Casiglia, A. Hofman, V. Salomaa, E. Barrett-Connor, R. Clarke, E. Brunner, J.W. Jukema, L.A. Simons, M. Sandhu, N.J. Wareham, K.T. Khaw, J. Kauhanen, JT. Salonen, W.J. Howard, B.G. Nordestgaard, A.M. Wood, S.G. Thompson, S.M. Boekholdt, N. Sattar, C. Packard, V. Gudnason, J. Danesh, Lipid-related markers and cardiovascular disease prediction, J. Am. Med. Assoc. 307 (2012) 2499-2506.

[46] C.J. Willer, E.M. Schmidt, S. Sengupta, G.M. Peloso, S. Gustafsson, S. Kanoni, A. Ganna, J. Chen, M.L. Buchkovich, S. Mora, J.S. Beckmann, J.L. Bragg-Gresham, H.Y. Chang, A. Demirkan, H.M. Den Hertog, R. Do, L.A. Donnelly, G.B. Ehret, T. Esko, M.F. Feitosa, T. Ferreira, K. Fischer, P. Fontanillas, R.M. Fraser, D.F. Freitag, D. Gurdasani, K. Heikkila, E. Hypponen, A. Isaacs, A.U. Jackson, A. Johansson, T. Johnson, M. Kaakinen, J. Kettunen, M.E. Kleber, X. Li, J. Luan, L.P. Lyytikainen, P.K.E. Magnusson, M. Mangino, E. Mihailov, M.E. Montasser, M. Muller-Nurasyid, I.M. Nolte, J.R. O'Connell, C.D. Palmer, M. Perola, A.K. Petersen, S. Sanna, R. Saxena, S.K. Service, S. Shah, D. Shungin, C. Sidore, C. Song, R.J. Strawbridge, I. Surakka, T. Tanaka, T.M. Teslovich, G. Thorleifsson, E.G. Van den Herik, B.F. Voight, K.A. Volcik, L.L. Waite, A. Wong, Y. Wu, W. Zhang, D. Absher, G. Asiki, I. Barroso, L.F. Been, J.L. Bolton, L.L. Bonnycastle, P. Brambilla, M.S. Burnett, G. Cesana, M. Dimitriou, A.S.F. Doney, A. Doring, P. Elliott, S.E. Epstein, G. Ingi Eyjolfsson, B. Gigante, M.O. Goodarzi, H. Grallert, M.L. Gravito, C.J. Groves, G. Hallmans, A.L. Hartikainen, C. Hayward, D. Hernandez, A.A. Hicks, H. Holm, Y.J. Hung, T. Illig, M.R. Jones, P. Kaleebu, J.J.P. Kastelein, K.T. Khaw, E. Kim, N. Klopp, P. Komulainen, M. Kumari, C. Langenberg, T. Lehtimaki, S.Y. Lin, J. Lindstrom, R.J.F. Loos, F. Mach, 
W.L. McArdle, C. Meisinger, B.D. Mitchell, G. Muller, R. Nagaraja, N. Narisu, T.V.M. Nieminen, R.N. Nsubuga, I. Olafsson, K.K. Ong, A. Palotie, T. Papamarkou, C. Pomilla, A. Pouta, D.J. Rader, M.P. Reilly, P.M. Ridker, F. Rivadeneira, I. Rudan, A. Ruokonen, N. Samani, H. Scharnagl, J. Seeley, K. Silander, A. Stancakova, K. Stirrups, A.J. Swift, L. Tiret, A.G. Uitterlinden, L.J. van Pelt, S. Vedantam, N. Wainwright, C. Wijmenga, S.H. Wild, G. Willemsen, T. Wilsgaard, J.F. Wilson, E.H. Young, J.H. Zhao, L.S. Adair, D. Arveiler, T.L. Assimes, S. Bandinelli, F. Bennett, M. Bochud, B.O. Boehm, D.I. Boomsma, I.B. Borecki, S.R. Bornstein, P. Bovet, M. Burnier, H. Campbell, A. Chakravarti, J.C. Chambers, Y.I. Chen, F.S. Collins, R.S. Cooper, J. Danesh, G. Dedoussis, U. de Faire, A.B. Feranil, J. Ferrieres, L. Ferrucci, N.B. Freimer, C. Gieger, L.C. Groop, V. Gudnason, U. Gyllensten, A. Hamsten, T.B. Harris, A. Hingorani, J.N. Hirschhorn, A. Hofman, G.K. Hovingh, C.A. Hsiung, S.E. Humphries, S.C. Hunt, K. Hveem, C. Iribarren, M.R. Jarvelin, A. Jula, M. Kahonen, J. Kaprio, A. Kesaniemi, M. Kivimaki, J.S. Kooner, P.J. Koudstaal, R.M. Krauss, D. Kuh, J. Kuusisto, K.O. Kyvik, M. Laakso, T.A. Lakka, L. Lind, C.M. Lindgren, N.G. Martin, W. Marz, M.I. McCarthy, C.A. McKenzie, P. Meneton, A. Metspalu, L. Moilanen, A.D. Morris, P.B. Munroe, I. Njolstad, N.L. Pedersen, C. Power, P.P. Pramstaller, J.F. Price, B.M. Psaty, T. Quertermous, R. Rauramaa, D. Saleheen, V. Salomaa, D.K. Sanghera, J. Saramies, P.E.H. Schwarz, W.H. Sheu, A.R. Shuldiner, A. Siegbahn, T.D. Spector, K. Stefansson, D.P. Strachan, B.O. Tayo, E. Tremoli, J. Tuomilehto, M. Uusitupa, C.M. van Duijn, P. Vollenweider, L. Wallentin, N.J. Wareham, J.B. Whitfield, B.H.R. Wolffenbuttel, J.M. Ordovas, E. Boerwinkle, C.N.A. Palmer, U. Thorsteinsdottir, D.I. Chasman, J.I. Rotter, P.W. Franks, S. Ripatti, L.A. Cupples, M.S. Sandhu, S.S. Rich, M. Boehnke, P. Deloukas, S. Kathiresan, K.L. Mohlke, E. Ingelsson, G.R. Abecasis, Global Lipids Genetics Consortium. Discovery and refinement of loci associated with lipid levels, Nat. Genet. 45 (2013) 1274-1283.

[47] M. Nikpay, A. Goel, H.H. Won, L.M. Hall, C. Willenborg, S. Kanoni, D. Saleheen, T. Kyriakou, C.P. Nelson, J.C. Hopewell, T.R. Webb, L. Zeng, A. Dehghan, M. Alver, S.M. Armasu, K. Auro, A. Bjonnes, D.I. Chasman, S. Chen, I. Ford, N. Franceschini, C. Gieger, C. Grace, S. Gustafsson, J. Huang, S.J. Hwang, Y.K. Kim, M.E. Kleber, K.W. Lau, X. Lu, Y. Lu, L.P. Lyytikainen, E. Mihailov, A.C. Morrison, N. Pervjakova, L. Qu, L.M. Rose, E. Salfati, R. Saxena, M. Scholz, A.V. Smith, E. Tikkanen, A. Uitterlinden, X. Yang, W. Zhang, W. Zhao, M. de Andrade, P.S. de Vries, N.R. van Zuydam, S.S. Anand, L. Bertram, F. Beutner, G. Dedoussis, P. Frossard, D. Gauguier, A.H. Goodall, O. Gottesman, M. Haber, B.G. Han, J. Huang, S. Jalilzadeh, T. Kessler, I.R. Konig, L. Lannfelt, W. Lieb, L. Lind, C.M. Lindgren, M.L. Lokki, P.K. Magnusson, N.H. Mallick, N. Mehra, T. Meitinger, F.U. Memon, A.P. Morris, M.S. Nieminen, N.L. Pedersen, A. Peters, L.S. Rallidis, A. Rasheed, M. Samuel, S.H. Shah, J. Sinisalo, K.E. Stirrups, S. Trompet, L. Wang, K.S. Zaman, D. Ardissino, E. Boerwinkle, I.B. Borecki, E.P. Bottinger, J.E. Buring, J.C. Chambers, R. Collins, L.A. Cupples, J. Danesh, I. Demuth, R. Elosua, S.E. Epstein, T. Esko, M.F. Feitosa, O.H. Franco, M.G. Franzosi, C.B. Granger, D. Gu, V. Gudnason, A.S. Hall, A. Hamsten, T.B. Harris, S.L. Hazen, C. Hengstenberg, A. Hofman, E. Ingelsson, C. Iribarren, J.W. Jukema, P.J. Karhunen, B.J. Kim, J.S. Kooner, I.J. Kullo, T. Lehtimaki, R.J.F. Loos, O. Melander, A. Metspalu, W. Marz, C.N. Palmer, M. Perola, T. Quertermous, D.J. Rader, P.M. Ridker, S. Ripatti, R. Roberts, V. Salomaa, D.K. Sanghera, S.M. Schwartz, U. Seedorf, A.F. Stewart, D.J. Stott, J. Thiery, P.A. Zalloua, C.J. O'Donnell, M.P. Reilly, T.L. Assimes, J.R. Thompson, J. Erdmann, R. Clarke, H. Watkins, S. Kathiresan, R. McPherson, P. Deloukas, H. Schunkert, N.J. Samani, M. Farrall, A comprehensive 1,000 Genomes-based genome-wide association meta-analysis of coronary artery disease, Nat. Genet. 47 (2015) 1121-1130.

[48] B.A. Ference, W. Yoo, I. Alesh, N. Mahajan, K.K. Mirowska, A. Mewada, J. Kahn, L. Afonso, K.A. Williams Sr., J.M. Flack, Effect of long-term exposure to lower lowdensity lipoprotein cholesterol beginning early in life on the risk of coronary heart disease: a Mendelian randomization analysis, J. Am. Coll. Cardiol. 60 (2012) 2631-2639.

[49] M.V. Holmes, F.W. Asselbergs, T.M. Palmer, F. Drenos, M.B. Lanktree, C.P. Nelson, C.E. Dale, S. Padmanabhan, C. Finan, D.I. Swerdlow, V. Tragante, E.P. van Iperen, S. Sivapalaratnam, S. Shah, C.C. Elbers, T. Shah, J. Engmann, C. Giambartolomei, J. White, D. Zabaneh, R. Sofat, S. McLachlan, U. consortium, P.A. Doevendans, A.J. Balmforth, A.S. Hall, K.E. North, B. Almoguera, R.C. Hoogeveen, M. Cushman, M. Fornage, S.R. Patel, S. Redline, D.S. Siscovick, M.Y. Tsai, K.J. Karczewski, M.H. Hofker, W.M. Verschuren, M.L. Bots, Y.T. van der Schouw, O. Melander, A.F. Dominiczak, R. Morris, Y. Ben-Shlomo, J. Price, M. Kumari, J. Baumert, A. Peters, B. Thorand, W. Koenig, T.R. Gaunt, S.E. Humphries, R. Clarke, H. Watkins, M. Farrall, J.G. Wilson, S.S. Rich, P.I. de Bakker, L.A. Lange, G. Davey Smith, A.P. Reiner, P.J. Talmud, M. Kivimaki, D.A. Lawlor, F. Dudbridge, N.J. Samani, B.J. Keating, A.D. Hingorani, J.P. Casas, Mendelian randomization of blood lipids for coronary heart disease, Eur. Heart J. 36 (2015) 539-550.

[50] M.G. Silverman, B.A. Ference, K. Im, S.D. Wiviott, R.P. Giugliano, S.M. Grundy, E. Braunwald, M.S. Sabatine, Association between lowering LDL-C and cardiovascular risk reduction among different therapeutic interventions: a systematic review and meta-analysis, J. Am. Med. Assoc. 316 (2016) 1289-1297.

[51] C. Baigent, A. Keech, P.M. Kearney, L. Blackwell, G. Buck, C. Pollicino, A. Kirby, T. Sourjina, R. Peto, R. Collins, R. Simes, Cholesterol Treatment Trialists' (CTT) Collaborators, Efficacy and safety of cholesterol-lowering treatment: prospective meta-analysis of data from 90,056 participants in 14 randomised trials of statins, Lancet 366 (2005) 1267-1278.

[52] J.C. Cohen, E. Boerwinkle, T.H. Mosley Jr., H.H. Hobbs, Sequence variations in PCSK9, low LDL, and protection against coronary heart disease, N. Engl. J. Med. 354 (2006) 1264-1272.

[53] B.A. Ference, J.J.P. Kastelein, K.K. Ray, H.N. Ginsberg, M.J. Chapman,
C.J. Packard, U. Laufs, C. Oliver-Williams, A.M. Wood, A.S. Butterworth, E. Di Angelantonio, J. Danesh, S.J. Nicholls, D.L. Bhatt, M.S. Sabatine, A.L. Catapano, Association of triglyceride-lowering LPL variants and LDL-C-lowering LDLR variants with risk of coronary heart disease, J. Am. Med. Assoc. 321 (2019) 364-373.

[54] B.A. Ference, F. Majeed, R. Penumetcha, J.M. Flack, R.D. Brook, Effect of naturally random allocation to lower low-density lipoprotein cholesterol on the risk of coronary heart disease mediated by polymorphisms in NPC1L1, HMGCR, or both: a 2 x 2 factorial Mendelian randomization study, J. Am. Coll. Cardiol. 65 (2015) $1552-1561$.

[55] B.A. Ference, J.G. Robinson, R.D. Brook, A.L. Catapano, M.J. Chapman, D.R. Neff, S. Voros, R.P. Giugliano, G. Davey Smith, S. Fazio, M.S. Sabatine, Variation in PCSK9 and HMGCR and risk of cardiovascular disease and diabetes, N. Engl. J. Med. 375 (2016) 2144-2153.

[56] Triglyceride Coronary Disease Genetics Consortium, Emerging Risk Factors Collaboration, N. Sarwar, M.S. Sandhu, S.L. Ricketts, A.S. Butterworth, E. Di Angelantonio, S.M. Boekholdt, W. Ouwehand, H. Watkins, N.J. Samani, D. Saleheen, D. Lawlor, M.P. Reilly, A.D. Hingorani, P.J. Talmud, J. Danesh, Triglyceride-mediated pathways and coronary disease: collaborative analysis of 101 studies, Lancet 375 (2010) 1634-1639.

[57] A. Varbo, M. Benn, A. Tybjaerg-Hansen, A.B. Jorgensen, R. Frikke-Schmidt, B.G. Nordestgaard, Remnant cholesterol as a causal risk factor for ischemic heart disease, J. Am. Coll. Cardiol. 61 (2013) 427-436.

[58] G.F. Lewis, C. Xiao, R.A. Hegele, Hypertriglyceridemia in the genomic era: a new paradigm, Endocr. Rev. 36 (2015) 131-147.

[59] J.S. Dron, R.A. Hegele, Complexity of mechanisms among human proprotein convertase subtilisin-kexin type 9 variants, Curr. Opin. Lipidol. 28 (2017) 161-169.

[60] Prospective Studies Collaboration, S. Lewington, G. Whitlock, R. Clarke, P. Sherliker, J. Emberson, J. Halsey, N. Qizilbash, R. Peto, R. Collins, Blood cholesterol and vascular mortality by age, sex, and blood pressure: a meta-analysis of individual data from 61 prospective studies with 55,000 vascular deaths, Lancet 370 (2007) 1829-1839.

[61] R. Frikke-Schmidt, B.G. Nordestgaard, M.C. Stene, A.A. Sethi, A.T. Remaley, P. Schnohr, P. Grande, A. Tybjaerg-Hansen, Association of loss-of-function mutations in the ABCA1 gene with high-density lipoprotein cholesterol levels and risk of ischemic heart disease, J. Am. Med. Assoc. 299 (2008) 2524-2532.

[62] B.F. Voight, G.M. Peloso, M. Orho-Melander, R. Frikke-Schmidt, M. Barbalic, M.K. Jensen, G. Hindy, H. Holm, E.L. Ding, T. Johnson, H. Schunkert, N.J. Samani, R. Clarke, J.C. Hopewell, J.F. Thompson, M. Li, G. Thorleifsson, C. Newton-Cheh, K. Musunuru, J.P. Pirruccello, D. Saleheen, L. Chen, A. Stewart, A. Schillert, U. Thorsteinsdottir, G. Thorgeirsson, S. Anand, J.C. Engert, T. Morgan, J. Spertus, M. Stoll, K. Berger, N. Martinelli, D. Girelli, P.P. McKeown, C.C. Patterson, S.E. Epstein, J. Devaney, M.S. Burnett, V. Mooser, S. Ripatti, I. Surakka, M.S. Nieminen, J. Sinisalo, M.L. Lokki, M. Perola, A. Havulinna, U. de Faire, B. Gigante, E. Ingelsson, T. Zeller, P. Wild, P.I. de Bakker, O.H. Klungel, A.H. Maitland-van der Zee, B.J. Peters, A. de Boer, D.E. Grobbee, P.W. Kamphuisen, V.H. Deneer, C.C. Elbers, N.C. Onland-Moret, M.H. Hofker, C. Wijmenga, W.M. Verschuren, J.M. Boer, Y.T. van der Schouw, A. Rasheed, P. Frossard, S. Demissie, C. Willer, R. Do, J.M. Ordovas, G.R. Abecasis, M. Boehnke, K.L. Mohlke, M.J. Daly, C. Guiducci, N.P. Burtt, A. Surti, E. Gonzalez, S. Purcell, S. Gabriel, J. Marrugat, J. Peden, J. Erdmann, P. Diemert, C. Willenborg, I.R. Konig, M. Fischer, C. Hengstenberg, A. Ziegler, I. Buysschaert, D. Lambrechts, F. Van de Werf, K.A. Fox, N.E. El Mokhtari, D. Rubin, J. Schrezenmeir, S. Schreiber, A. Schafer, J. Danesh, S. Blankenberg, R. Roberts, R. McPherson, H. Watkins, A.S. Hall, K. Overvad, E. Rimm, E. Boerwinkle, A. Tybjaerg-Hansen, L.A. Cupples, M.P. Reilly, O. Melander, P.M. Mannucci, D. Ardissino, D. Siscovick, R. Elosua, K. Stefansson, C.J. O'Donnell, V. Salomaa, D.J. Rader, L. Peltonen, S.M. Schwartz, D. Altshuler, S. Kathiresan, Plasma HDL cholesterol and risk of myocardial infarction: a Mendelian randomisation study, Lancet 380 (2012) 572-580.

[63] A.M. Lincoff, S.J. Nicholls, J.S. Riesmeyer, P.J. Barter, H.B. Brewer, K.A.A. Fox, C.M. Gibson, C. Granger, V. Menon, G. Montalescot, D. Rader, A.R. Tall, E. McErlean, K. Wolski, G. Ruotolo, B. Vangerow, G. Weerakkody, S.G. Goodman, D. Conde, D.K. McGuire, J.C. Nicolau, J.L. Leiva-Pons, Y. Pesant, W. Li, D. Kandath, S. Kouz, N. Tahirkheli, D. Mason, S.E. Nissen, ACCELERATE Investigators. Evacetrapib and cardiovascular outcomes in high-risk vascular disease, N. Engl. J. Med. 376 (2017) 1933-1942.

[64] HPS/TIMI/REVEAL Collaborative Group, L. Bowman, J.C. Hopewell, F. Chen, K. Wallendszus, W. Stevens, R. Collins, S.D. Wiviott, C.P. Cannon, E. Braunwald, E. Sammons, M.J. Landray, Effects of anacetrapib in patients with atherosclerotic vascular disease, N. Engl. J. Med. 377 (2017) 1217-1227.

[65] G.G. Schwartz, A.G. Olsson, M. Abt, C.M. Ballantyne, P.J. Barter, J. Brumm, B.R. Chaitman, I.M. Holme, D. Kallend, L.A. Leiter, E. Leitersdorf, J.J. McMurray, H. Mundl, S.J. Nicholls, P.K. Shah, J.C. Tardif, R.S. Wright, dalOUTCOMES. Investigators, Effects of dalcetrapib in patients with a recent acute coronary syndrome, N. Engl. J. Med. 367 (2012) 2089-2099.

[66] Aim-High Investigators, W.E. Boden, J.L. Probstfield, T. Anderson, B.R. Chaitman, P. Desvignes-Nickens, K. Koprowicz, R. McBride, K. Teo, W. Weintraub, Niacin in patients with low HDL cholesterol levels receiving intensive statin therapy, N. Engl. J. Med. 365 (2011) 2255-2267.

[67] H.T.C. Group, M.J. Landray, R. Haynes, J.C. Hopewell, S. Parish, T. Aung, J. Tomson, K. Wallendszus, M. Craig, L. Jiang, R. Collins, J. Armitage, Effects of extended-release niacin with laropiprant in high-risk patients, N. Engl. J. Med. 371 (2014) 203-212.

[68] J. Andrews, A. Janssan, T. Nguyen, A.D. Pisaniello, D.J. Scherer, J.J. Kastelein, B. Merkely, S.E. Nissen, K. Ray, G.G. Schwartz, S.G. Worthley, C. Keyserling, 
J.L. Dasseux, J. Butters, J. Girardi, R. Miller, S.J. Nicholls, Effect of serial infusions of reconstituted high-density lipoprotein (CER-001) on coronary atherosclerosis: rationale and design of the CARAT study, Cardiovasc. Diagn. Ther. 7 (2017) 45-51.

[69] J.C. Tardif, C.M. Ballantyne, P. Barter, J.L. Dasseux, Z.A. Fayad, M.C. Guertin, J.J. Kastelein, C. Keyserling, H. Klepp, W. Koenig, P.L. L'Allier, J. Lesperance, T.F. Luscher, J.F. Paolini, A. Tawakol, D.D. Waters, Can HDL Infusions Significantly QUicken Atherosclerosis REgression (CHI-SQUARE) Investigators. Effects of the high-density lipoprotein mimetic agent CER-001 on coronary atherosclerosis in patients with acute coronary syndromes: a randomized trial, Eur. Heart J. 35 (2014) 3277-3286.

[70] B.G. Nordestgaard, A. Langsted, Lipoprotein (a) as a cause of cardiovascular disease: insights from epidemiology, genetics, and biology, J. Lipid Res. 57 (2016) 1953-1975.

[71] F.M. van der Valk, S. Bekkering, J. Kroon, C. Yeang, J. Van den Bossche, J.D. van Buul, A. Ravandi, A.J. Nederveen, H.J. Verberne, C. Scipione, M. Nieuwdorp, L.A. Joosten, M.G. Netea, M.L. Koschinsky, J.L. Witztum, S. Tsimikas, N.P. Riksen, E.S. Stroes, Oxidized phospholipids on lipoprotein(a) elicit arterial wall inflammation and an inflammatory monocyte response in humans, Circulation 134 (2016) 611-624.

[72] B.G. Nordestgaard, M.J. Chapman, K. Ray, J. Borén, F. Andreotti, G.F. Watts, H. Ginsberg, P. Amarenco, A. Catapano, O.S. Descamps, E. Fisher, P.T. Kovanen, J.A. Kuivenhoven, P. Lesnik, L. Masana, Z. Reiner, M.R. Taskinen, L. Tokgözoglu, A. Tybjærg-Hansen, European Atherosclerosis Society Consensus Panel. Lipoprotein(a) as a cardiovascular risk factor: current status, Eur. Heart J. 31 (2010) 2844-2853.

[73] Emerging Risk Factors Collaboration, S. Erqou, S. Kaptoge, P.L. Perry, E. Di Angelantonio, A. Thompson, I.R. White, S.M. Marcovina, R. Collins, S.G. Thompson, J. Danesh, Lipoprotein(a) concentration and the risk of coronary heart disease, stroke, and nonvascular mortality, J. Am. Med. Assoc. 302 (2009) 412-423.

[74] R. Clarke, J.F. Peden, J.C. Hopewell, T. Kyriakou, A. Goel, S.C. Heath, S. Parish, S. Barlera, M.G. Franzosi, S. Rust, D. Bennett, A. Silveira, A. Malarstig, F.R. Green, M. Lathrop, B. Gigante, K. Leander, U. de Faire, U. Seedorf, A. Hamsten, R. Collins, H. Watkins, M. Farrall, PROCARDIS Consortium, Genetic variants associated with Lp(a) lipoprotein level and coronary disease, N. Engl. J. Med. 361 (2009) $2518-2528$.

[75] P.R. Kamstrup, A. Tybjaerg-Hansen, R. Steffensen, B.G. Nordestgaard, Genetically elevated lipoprotein(a) and increased risk of myocardial infarction, J. Am. Med. Assoc. 301 (2009) 2331-2339.

[76] M.L. O'Donoghue, S. Fazio, R.P. Giugliano, E.S.G. Stroes, E. Kanevsky, I. GouniBerthold, K. Im, A. Lira Pineda, S.M. Wasserman, R. Ceska, M.V. Ezhov, J.W. Jukema, H.K. Jensen, S.L. Tokgozoglu, F. Mach, K. Huber, P.S. Sever, A.C. Keech, T.R. Pedersen, M.S. Sabatine, Lipoprotein(a), PCSK9 inhibition, and cardiovascular risk, Circulation 139 (2019) 1483-1492.

[77] S. Burgess, B.A. Ference, J.R. Staley, D.F. Freitag, A.M. Mason, S.F. Nielsen, P. Willeit, R. Young, P. Surendran, S. Karthikeyan, T.R. Bolton, J.E. Peters, P.R. Kamstrup, A. Tybjaerg-Hansen, M. Benn, A. Langsted, P. Schnohr, S. VedelKrogh, C.J. Kobylecki, I. Ford, C. Packard, S. Trompet, J.W. Jukema, N. Sattar, E. Di Angelantonio, D. Saleheen, J.M.M. Howson, B.G. Nordestgaard, A.S. Butterworth, J. Danesh, European Prospective Investigation into Cancer and Nutrition-Cardiovascular Disease (EPIC-CVD) Consortium. Association of LPA variants with risk of coronary disease and the implications for lipoprotein(a) lowering therapies: a Mendelian randomization analysis, JAMA Cardiol 3 (2018) 619-627.

[78] S. Parish, J.C. Hopewell, M.R. Hill, S. Marcovina, E. Valdes-Marquez, R. Haynes, A. Offer, T.R. Pedersen, C. Baigent, R. Collins, M. Landray, J. Armitage, HPS2THRIVE Collaborative Group, Impact of apolipoprotein(a) isoform size on lipoprotein(a) lowering in the HPS2-THRIVE Study, Circ Genom Precis Med 11 (2018) e001696.

[79] M.R. Langlois, M.J. Chapman, C. Cobbaert, S. Mora, A.T. Remaley, E. Ros, G.F. Watts, J. Borén, H. Baum, E. Bruckert, A. Catapano, O.S. Descamps, A. von Eckardstein, P.R. Kamstrup, G. Kolovou, F. Kronenberg, A. Langsted, K. Pulkki, N. Rifai, G. Sypniewska, O. Wiklund, B.G. Nordestgaard, European Atherosclerosis Society (EAS) and the European Federation of Clinical Chemistry and Laboratory Medicine (EFLM) Joint Consensus Initiative. Quantifying atherogenic lipoproteins: current and future challenges in the era of personalized medicine and very low concentrations of LDL cholesterol. A consensus statement from EAS and EFLM, Clin. Chem. 64 (2018) 1006-1033.

[80] I. Jialal, M. Inn, D. Siegel, S. Devaraj, Underestimation of low density lipoproteincholesterol with the Friedewald equation versus a direct homogenous low density lipoprotein-cholesterol assay, Lab. Med. 48 (2017) 220-224.

[81] S.S. Martin, M.J. Blaha, M.B. Elshazly, E.A. Brinton, P.P. Toth, J.W. McEvoy, P.H. Joshi, K.R. Kulkarni, P.D. Mize, P.O. Kwiterovich, A.P. Defilippis, R.S. Blumenthal, S.R. Jones, Friedewald-estimated versus directly measured lowdensity lipoprotein cholesterol and treatment implications, J. Am. Coll. Cardiol. 62 (2013) 732-739.

[82] M. Nauck, G.R. Warnick, N. Rifai, Methods for measurement of LDL-cholesterol: a critical assessment of direct measurement by homogeneous assays versus calculation, Clin. Chem. 48 (2002) 236-254.

[83] F. Razi, K. Forouzanfar, F. Bandarian, E. Nasli-Esfahani, LDL-cholesterol measurement in diabetic type 2 patients: a comparison between direct assay and popular equations, J. Diabetes Metab. Disord. 16 (2017) 43.

[84] J.G. Robinson, R.S. Rosenson, M. Farnier, U. Chaudhari, W.J. Sasiela, L. Merlet, K. Miller, J.J. Kastelein, Safety of very low low-density lipoprotein cholesterol levels with alirocumab: pooled data from randomized trials, J. Am. Coll. Cardiol.
69 (2017) 471-482.

[85] V. Sathiyakumar, J. Park, A. Golozar, M. Lazo, R. Quispe, E. Guallar, R.S. Blumenthal, S.R. Jones, S.S. Martin, Fasting versus nonfasting and low-density lipoprotein cholesterol accuracy, Circulation 137 (2018) 10-19.

[86] S.P. Whelton, J.W. Meeusen, L.J. Donato, A.S. Jaffe, A. Saenger, L.J. Sokoll, R.S. Blumenthal, S.R. Jones, S.S. Martin, Evaluating the atherogenic burden of individuals with a Friedewald-estimated low-density lipoprotein cholesterol $<70$ $\mathrm{mg} / \mathrm{dL}$ compared with a novel low-density lipoprotein estimation method, J Clin Lipidol 11 (2017) 1065-1072.

[87] J.W. Meeusen, A.J. Lueke, A.S. Jaffe, A.K. Saenger, Validation of a proposed novel equation for estimating LDL cholesterol, Clin. Chem. 60 (2014) 1519-1523.

[88] M.R. Langlois, O.S. Descamps, A. van der Laarse, C. Weykamp, H. Baum, K. Pulkki, A. von Eckardstein, D. De Bacquer, J. Borén, O. Wiklund, P. Laitinen, W.P. Oosterhuis, C. Cobbaert, EAS-EFLM Collaborative Project, Clinical impact of direct HDLc and LDLc method bias in hypertriglyceridemia. A simulation study of the EAS-EFLM Collaborative Project Group, Atherosclerosis 233 (2014) 83-90.

[89] T. Miida, K. Nishimura, T. Okamura, S. Hirayama, H. Ohmura, H. Yoshida, Y. Miyashita, M. Ai, A. Tanaka, H. Sumino, M. Murakami, I. Inoue, Y. Kayamori, M. Nakamura, T. Nobori, Y. Miyazawa, T. Teramoto, S. Yokoyama, A multicenter study on the precision and accuracy of homogeneous assays for LDL-cholesterol: comparison with a beta-quantification method using fresh serum obtained from non-diseased and diseased subjects, Atherosclerosis 225 (2012) 208-215.

[90] W.G. Miller, G.L. Myers, I. Sakurabayashi, L.M. Bachmann, S.P. Caudill, A. Dziekonski, S. Edwards, M.M. Kimberly, W.J. Korzun, E.T. Leary, K. Nakajima, M. Nakamura, G. Nilsson, R.D. Shamburek, G.W. Vetrovec, G.R. Warnick, A.T. Remaley, Seven direct methods for measuring HDL and LDL cholesterol compared with ultracentrifugation reference measurement procedures, Clin. Chem. 56 (2010) 977-986.

[91] S.M. Marcovina, J.J. Albers, Lipoprotein (a) measurements for clinical application, J. Lipid Res. 57 (2016) 526-537.

[92] S.M. Marcovina, M.L. Koschinsky, J.J. Albers, S. Skarlatos, Report of the national heart, lung, and blood institute workshop on lipoprotein(a) and cardiovascular disease: recent advances and future directions, Clin. Chem. 49 (2003) 1785-1796.

[93] S. Tsimikas, S. Fazio, N.J. Viney, S. Xia, J.L. Witztum, S.M. Marcovina, Relationship of lipoprotein(a) molar concentrations and mass according to lipoprotein(a) thresholds and apolipoprotein(a) isoform size, J Clin Lipidol 12 (2018) $1313-1323$

[94] S. Mora, N. Rifai, J.E. Buring, P.M. Ridker, Fasting compared with nonfasting lipids and apolipoproteins for predicting incident cardiovascular events, Circulation 118 (2008) 993-1001.

[95] A.B. Jorgensen, R. Frikke-Schmidt, A.S. West, P. Grande, B.G. Nordestgaard, A. Tybjaerg-Hansen, Genetically elevated non-fasting triglycerides and calculated remnant cholesterol as causal risk factors for myocardial infarction, Eur. Heart J. 34 (2013) 1826-1833.

[96] G.D. Kolovou, D.P. Mikhailidis, J. Kovar, D. Lairon, B.G. Nordestgaard, T.C. Ooi, P. Perez-Martinez, H. Bilianou, K. Anagnostopoulou, G. Panotopoulos, Assessment and clinical relevance of non-fasting and postprandial triglycerides: an expert panel statement, Curr. Vasc. Pharmacol. 9 (2011) 258-270.

[97] C. Mihas, G.D. Kolovou, D.P. Mikhailidis, J. Kovar, D. Lairon, B.G. Nordestgaard, T.C. Ooi, P. Perez-Martinez, H. Bilianou, K. Anagnostopoulou, G. Panotopoulos, Diagnostic value of postprandial triglyceride testing in healthy subjects: a metaanalysis, Curr. Vasc. Pharmacol. 9 (2011) 271-280.

[98] B.G. Nordestgaard, A. Varbo, Triglycerides and cardiovascular disease, Lancet 384 (2014) 626-635.

[99] A.M. Baca, G.R. Warnick, Estimation of LDL-associated apolipoprotein B from measurements of triglycerides and total apolipoprotein B, Clin. Chem. 54 (2008) 907-910.

[100] M.J. Chapman, H.N. Ginsberg, P. Amarenco, F. Andreotti, J. Borén, A.L. Catapano, O.S. Descamps, E. Fisher, P.T. Kovanen, J.A. Kuivenhoven, P. Lesnik, L. Masana, B.G. Nordestgaard, K.K. Ray, Z. Reiner, M.R. Taskinen, L. Tokgözoglu, A. Tybjærg-Hansen, G.F. Watts, European Atherosclerosis Society Consensus Panel. Triglyceride-rich lipoproteins and high-density lipoprotein cholesterol in patients at high risk of cardiovascular disease: evidence and guidance for management, Eur. Heart J. 32 (2011) 1345-1361.

[101] L.J. Cartier, C. Collins, M. Lagace, P. Douville, Comparison of fasting and nonfasting lipid profiles in a large cohort of patients presenting at a community hospital, Clin. Biochem. 52 (2018) 61-66.

[102] National Clinical Guideline Centre (UK), Lipid Modification: Cardiovascular Risk Assessment and the Modification of Blood Lipids for the Primary and Secondary Prevention of Cardiovascular Disease, National Institute for Health and Care Excellence, London, 2014 UK.

[103] Joint British Societies Board, Joint British Societies' consensus recommendations for the prevention of cardiovascular disease (JBS3), Heart 100 (2014) ii1-ii67.

[104] B. Doran, Y. Guo, J. Xu, H. Weintraub, S. Mora, D.J. Maron, S. Bangalore, Prognostic value of fasting versus nonfasting low-density lipoprotein cholesterol levels on long-term mortality: insight from the National Health and Nutrition Examination Survey III (NHANES-III), Circulation 130 (2014) 546-553.

[105] G. Harari, M.S. Green, A. Magid, S. Zelber-Sagi, Usefulness of non-high-density lipoprotein cholesterol as a predictor of cardiovascular disease mortality in men in 22-year follow-up, Am. J. Cardiol. 119 (2017) 1193-1198.

[106] X. Gu, X. Yang, Y. Li, J. Cao, J. Li, X. Liu, J. Chen, C. Shen, L. Yu, J. Huang, D. Gu, Usefulness of low-density lipoprotein cholesterol and non-high-density lipoprotein cholesterol as predictors of cardiovascular disease in Chinese, Am. J. Cardiol. 116 (2015) 1063-1070.

[107] M.J. van den Berg, Y. van der Graaf, G.J. de Borst, L.J. Kappelle, H.M. Nathoe, F.L.J. Visseren, SMART Study Group, Low-density lipoprotein cholesterol, non- 
high-density lipoprotein cholesterol, triglycerides, and apolipoprotein B and cardiovascular risk in patients with manifest arterial disease, Am. J. Cardiol. 118 (2016) 804-810.

[108] H.E. van Deventer, W.G. Miller, G.L. Myers, I. Sakurabayashi, L.M. Bachmann, S.P. Caudill, A. Dziekonski, S. Edwards, M.M. Kimberly, W.J. Korzun, E.T. Leary, K. Nakajima, M. Nakamura, R.D. Shamburek, G.W. Vetrovec, G.R. Warnick, A.T. Remaley, Non-HDL cholesterol shows improved accuracy for cardiovascular risk score classification compared to direct or calculated LDL cholesterol in a dyslipidemic population, Clin. Chem. 57 (2011) 490-501.

[109] A.D. Sniderman, S. Islam, S. Yusuf, M.J. McQueen, Discordance analysis of apolipoprotein B and non-high density lipoprotein cholesterol as markers of cardiovascular risk in the INTERHEART study, Atherosclerosis 225 (2012) 444-449.

[110] N.R. Cook, S. Mora, P.M. Ridker, Lipoprotein(a) and cardiovascular risk prediction among women, J. Am. Coll. Cardiol. 72 (2018) 287-296.

[111] P.R. Kamstrup, A. Tybjaerg-Hansen, B.G. Nordestgaard, Extreme lipoprotein(a) levels and improved cardiovascular risk prediction, J. Am. Coll. Cardiol. 61 (2013) 1146-1156.

[112] P. Willeit, S. Kiechl, F. Kronenberg, J.L. Witztum, P. Santer, M. Mayr, Q. Xu, A. Mayr, J. Willeit, S. Tsimikas, Discrimination and net reclassification of cardi ovascular risk with lipoprotein(a): prospective 15-year outcomes in the Bruneck Study, J. Am. Coll. Cardiol. 64 (2014) 851-860.

[113] European Association for Cardiovascular Prevention \& Rehabilitation, Z. Reiner, A.L. Catapano, G. De Backer, I. Graham, M.R. Taskinen, O. Wiklund, S. Agewall, E. Alegria, M.J. Chapman, P. Durrington, S. Erdine, J. Halcox, R. Hobbs, J. Kjekshus, P.P. Filardi, G. Riccardi, R.F. Storey, D. Wood, ESC Committee for practice guidelines Committees (CPG) 2008-2010 and 2010-2012 Committees. ESC/EAS guidelines for the management of dyslipidaemias: the task force for the management of dyslipidaemias of the European society of Cardiology (ESC) and the European atherosclerosis society (EAS), Eur. Heart J. 32 (2011) 1769-1818.

[114] D.M. Lloyd-Jones, P.B. Morris, C.M. Ballantyne, K.K. Birtcher, D.D. Daly Jr., S.M. DePalma, M.B. Minissian, C.E. Orringer, S.C. Smith Jr., Focused update of the 2016 ACC expert consensus decision pathway on the role of non-statin therapies for LDL-cholesterol lowering in the management of atherosclerotic cardiovascular disease risk: a report of the American College of Cardiology task force on expert consensus decision pathways, J. Am. Coll. Cardiol. 70 (2017) 1785-1822 2017.

[115] E.P. Navarese, J.G. Robinson, M. Kowalewski, M. Kolodziejczak, F. Andreotti, K. Bliden, U. Tantry, J. Kubica, P. Raggi, P.A. Gurbel, Association between baseline LDL-C level and total and cardiovascular mortality after LDL-C lowering: a systematic review and meta-analysis, J. Am. Med. Assoc. 319 (2018) 1566-1579.

[116] J. Hsia, J.G. MacFadyen, J. Monyak, P.M. Ridker, Cardiovascular event reduction and adverse events among subjects attaining low-density lipoprotein cholesterol $<50 \mathrm{mg} / \mathrm{dl}$ with rosuvastatin. The JUPITER trial (Justification for the Use of Statins in Prevention: an Intervention Trial Evaluating Rosuvastatin), J. Am. Coll. Cardiol. 57 (2011) 1666-1675.

[117] T. McCormack, R. Dent, M. Blagden, Very low LDL-C levels may safely provide additional clinical cardiovascular benefit: the evidence to date, Int. J. Clin. Pract. 70 (2016) 886-897.

[118] B. Williams, G. Mancia, W. Spiering, E. Agabiti Rosei, M. Azizi, M. Burnier, D.L. Clement, A. Coca, G. de Simone, A. Dominiczak, T. Kahan, F. Mahfoud, J. Redon, L. Ruilope, A. Zanchetti, M. Kerins, S.E. Kjeldsen, R. Kreutz, S. Laurent, G.Y.H. Lip, R. McManus, K. Narkiewicz, F. Ruschitzka, R.E. Schmieder, E. Shlyakhto, C. Tsioufis, V. Aboyans, I. Desormais, Authors/Task Force Members, ESC/ESH guidelines for the management of arterial hypertension: the task force for the management of arterial hypertension of the European society of Cardiology and the European society of hypertension: the task force for the management of arterial hypertension of the European society of Cardiology and the European society of hypertension, J. Hypertens. 36 (2018) 1953-2041 2018.

[119] M.S. Sabatine, R.P. Giugliano, A.C. Keech, N. Honarpour, S.D. Wiviott, S.A. Murphy, J.F. Kuder, H. Wang, T. Liu, S.M. Wasserman, P.S. Sever, T.R. Pedersen, FOURIER Steering Committee and Investigators. Evolocumab and clinical outcomes in patients with cardiovascular disease, N. Engl. J. Med. 376 (2017) 1713-1722.

[120] G.G. Schwartz, P.G. Steg, M. Szarek, D.L. Bhatt, V.A. Bittner, R. Diaz, J.M. Edelberg, S.G. Goodman, C. Hanotin, R.A. Harrington, J.W. Jukema, G. Lecorps, K.W. Mahaffey, A. Moryusef, R. Pordy, K. Quintero, M.T. Roe, W.J. Sasiela, J.F. Tamby, P. Tricoci, H.D. White, A.M. Zeiher, ODYSSEY OUTCOMES Committees and Investigators. Alirocumab and cardiovascular outcomes after acute coronary syndrome, N. Engl. J. Med. 379 (2018) 2097-2107.

[121] V. Charlton-Menys, D.J. Betteridge, H. Colhoun, J. Fuller, M. France, G.A. Hitman, S.J. Livingstone, H.A. Neil, C.B. Newman, M. Szarek, D.A. DeMicco, P.N. Durrington, Targets of statin therapy: LDL cholesterol, non-HDL cholesterol, and apolipoprotein B in type 2 diabetes in the Collaborative Atorvastatin Diabetes Study (CARDS), Clin. Chem. 55 (2009) 473-480.

[122] A. Thompson, J. Danesh, Associations between apolipoprotein B, apolipoprotein $\mathrm{AI}$, the apolipoprotein $\mathrm{B} / \mathrm{AI}$ ratio and coronary heart disease: a literature-based meta-analysis of prospective studies, J. Intern. Med. 259 (2006) 481-492.

[123] G. Thanassoulis, K. Williams, K. Ye, R. Brook, P. Couture, P.R. Lawler, J. de Graaf, C.D. Furberg, A. Sniderman, Relations of change in plasma levels of LDL-C, nonHDL-C and apoB with risk reduction from statin therapy: a meta-analysis of randomized trials, J Am Heart Assoc 3 (2014) e000759.

[124] J.G. Robinson, S. Wang, B.J. Smith, T.A. Jacobson, Meta-analysis of the relationship between non-high-density lipoprotein cholesterol reduction and coronary heart disease risk, J. Am. Coll. Cardiol. 53 (2009) 316-322.

[125] J.E. Dalen, S. Devries, Diets to prevent coronary heart disease 1957-2013: what have we learned? Am. J. Med. 127 (2014) 364-369.

[126] R.H. Eckel, J.M. Jakicic, J.D. Ard, J.M. de Jesus, N. Houston Miller, V.S. Hubbard,
I.M. Lee, A.H. Lichtenstein, C.M. Loria, B.E. Millen, C.A. Nonas, F.M. Sacks, S.C. Smith Jr., L.P. Svetkey, T.A. Wadden, S.Z. Yanovski, K.A. Kendall, L.C. Morgan, M.G. Trisolini, G. Velasco, J. Wnek, J.L. Anderson, J.L. Halperin, N.M. Albert, B. Bozkurt, R.G. Brindis, L.H. Curtis, D. DeMets, J.S. Hochman, R.J. Kovacs, E.M. Ohman, S.J. Pressler, F.W. Sellke, W.K. Shen, S.C. Smith Jr., G.F. Tomaselli, American College of Cardiology/American heart association task force on practice guidelines. 2013 AHA/ACC guideline on lifestyle management to reduce cardiovascular risk: a report of the American College of Cardiology/ American heart association task force on practice guidelines, Circulation 129 (2014) S76-S99.

[127] A. Mente, L. de Koning, H.S. Shannon, S.S. Anand, A systematic review of the evidence supporting a causal link between dietary factors and coronary heart disease, Arch. Intern. Med. 169 (2009) 659-669.

[128] R. Chowdhury, S. Warnakula, S. Kunutsor, F. Crowe, H.A. Ward, L. Johnson, O.H. Franco, A.S. Butterworth, N.G. Forouhi, S.G. Thompson, K.T. Khaw, D. Mozaffarian, J. Danesh, E. Di Angelantonio, Association of dietary, circulating, and supplement fatty acids with coronary risk: a systematic review and metaanalysis, Ann. Intern. Med. 160 (2014) 398-406.

[129] D. Mozaffarian, R. Micha, S. Wallace, Effects on coronary heart disease of increasing polyunsaturated fat in place of saturated fat: a systematic review and meta-analysis of randomized controlled trials, PLoS Med. 7 (2010) e1000252.

[130] N.G. Forouhi, R.M. Krauss, G. Taubes, W. Willett, Dietary fat and cardiometabolic health: evidence, controversies, and consensus for guidance, BMJ 361 (2018) k2139.

[131] D. Mozaffarian, Natural trans fat, dairy fat, partially hydrogenated oils, and cardiometabolic health: the Ludwigshafen Risk and Cardiovascular Health Study, Eur. Heart J. 37 (2016) 1079-1081.

[132] M. Chen, Y. Li, Q. Sun, A. Pan, J.E. Manson, K.M. Rexrode, W.C. Willett, E.B. Rimm, F.B. Hu, Dairy fat and risk of cardiovascular disease in 3 cohorts of US adults, Am. J. Clin. Nutr. 104 (2016) 1209-1217.

[133] T.J. Moore, W.M. Vollmer, L.J. Appel, F.M. Sacks, L.P. Svetkey, T.M. Vogt, P.R. Conlin, D.G. Simons-Morton, L. Carter-Edwards, D.W. Harsha, Effect of dietary patterns on ambulatory blood pressure : results from the dietary approaches to stop hypertension (DASH) trial. DASH collaborative research group, Hypertension 34 (1999) 472-477.

[134] F. Sofi, C. Macchi, R. Abbate, G.F. Gensini, A. Casini, Mediterranean diet and health status: an updated meta-analysis and a proposal for a literature-based adherence score, Public Health Nutr. 17 (2014) 2769-2782.

[135] G. Grosso, S. Marventano, J. Yang, A. Micek, A. Pajak, L. Scalfi, F. Galvano, S.N. Kales, A comprehensive meta-analysis on evidence of Mediterranean diet and cardiovascular disease: are individual components equal? Crit. Rev. Food Sci. Nutr. 57 (2017) 3218-3232.

[136] M. de Lorgeril, P. Salen, J.L. Martin, I. Monjaud, J. Delaye, N. Mamelle, Mediterranean diet, traditional risk factors, and the rate of cardiovascular complications after myocardial infarction: final report of the Lyon Diet Heart Study, Circulation 99 (1999) 779-785.

[137] R. Estruch, E. Ros, J. Salas-Salvadó, M.I. Covas, D. Corella, F. Arós, E. GómezGracia, V. Ruiz-Gutiérrez, M. Fiol, J. Lapetra, R.M. Lamuela-Raventos, L. SerraMajem, X. Pintó, J. Basora, M.A. Muñoz, J.V. Sorlí, J.A. Martínez, M.A. MartínezGonzález, Retraction and republication: primary prevention of cardiovascular disease with a Mediterranean diet, N. Engl. J. Med. 378 (2018) 2441-2442.

[140] L. Brown, B. Rosner, W.W. Willett, F.M. Sacks, Cholesterol-lowering effects of dietary fiber: a meta-analysis, Am. J. Clin. Nutr. 69 (1999) 30-42.

[142] H. Gylling, J. Plat, S. Turley, H.N. Ginsberg, L. Ellegård, W. Jessup, P.J. Jones, D. Lütjohann, W. Maerz, L. Masana, G. Silbernagel, B. Staels, J. Borén, A.L. Catapano, G. De Backer, J. Deanfield, O.S. Descamps, P.T. Kovanen, G. Riccardi, L. Tokgözoglu, M.J. Chapman, European Atherosclerosis Society Consensus Panel on Phytosterols. Plant sterols and plant stanols in the management of dyslipidaemia and prevention of cardiovascular disease, Atherosclerosis 232 (2014) 346-360.

[143] K. Musa-Veloso, T.H. Poon, J.A. Elliot, C. Chung, A comparison of the LDL-cholesterol lowering efficacy of plant stanols and plant sterols over a continuous dose range: results of a meta-analysis of randomized, placebo-controlled trials, Prostaglandins Leukot. Essent. Fatty Acids 85 (2011) 9-28.

[144] A. Poli, C.M. Barbagallo, A.F.G. Cicero, A. Corsini, E. Manzato, B. Trimarco, F. Bernini, F. Visioli, A. Bianchi, G. Canzone, C. Crescini, S. de Kreutzenberg, N. Ferrara, M. Gambacciani, A. Ghiselli, C. Lubrano, G. Marelli, W. Marrocco, V. Montemurro, D. Parretti, R. Pedretti, F. Perticone, R. Stella, F. Marangoni, Nutraceuticals and functional foods for the control of plasma cholesterol levels. An intersociety position paper, Pharmacol. Res. 134 (2018) 51-60.

[145] Y. Li, L. Jiang, Z. Jia, W. Xin, S. Yang, Q. Yang, L. Wang, A meta-analysis of red yeast rice: an effective and relatively safe alternative approach for dyslipidemia, PLoS One 9 (2014) e98611.

[146] Z. Lu, W. Kou, B. Du, Y. Wu, S. Zhao, O.A. Brusco, J.M. Morgan, D.M. Capuzzi, Chinese Coronary Secondary Prevention Study Group, Li S. Effect of Xuezhikang, an extract from red yeast Chinese rice, on coronary events in a Chinese population with previous myocardial infarction, Am. J. Cardiol. 101 (2008) 1689-1693.

[148] E. Zomer, K. Gurusamy, R. Leach, C. Trimmer, T. Lobstein, S. Morris, W.P. James, N. Finer, Interventions that cause weight loss and the impact on cardiovascular risk factors: a systematic review and meta-analysis, Obes. Rev. 17 (2016) 1001-1011.

[151] K. Shaw, H. Gennat, P. O'Rourke, C. Del Mar, Exercise for overweight or obesity, Cochrane Database Syst. Rev. 4 (2006) CD003817.

[153] E.B. Rimm, P. Williams, K. Fosher, M. Criqui, M.J. Stampfer, Moderate alcohol intake and lower risk of coronary heart disease: meta-analysis of effects on lipids and haemostatic factors, BMJ 319 (1999) 1523-1528. 
[156] A.A. Rivellese, A. Maffettone, B. Vessby, M. Uusitupa, K. Hermansen, L. Berglund, A. Louheranta, B.J. Meyer, G. Riccardi, Effects of dietary saturated, monounsaturated and n-3 fatty acids on fasting lipoproteins, LDL size and post-prandial lipid metabolism in healthy subjects, Atherosclerosis 167 (2003) 149-158.

[157] M.Y. Wei, T.A. Jacobson, Effects of eicosapentaenoic acid versus docosahexaenoic acid on serum lipids: a systematic review and meta-analysis, Curr. Atheroscler. Rep. 13 (2011) 474-483.

[158] R. Kelishadi, M. Mansourian, M. Heidari-Beni, Association of fructose consumption and components of metabolic syndrome in human studies: a systematic review and meta-analysis, Nutrition 30 (2014) 503-510.

[159] K.L. Stanhope, J.M. Schwarz, N.L. Keim, S.C. Griffen, A.A. Bremer, J.L. Graham, B. Hatcher, C.L. Cox, A. Dyachenko, W. Zhang, J.P. McGahan, A. Seibert, R.M. Krauss, S. Chiu, E.J. Schaefer, M. Ai, S. Otokozawa, K. Nakajima, T. Nakano, C. Beysen, M.K. Hellerstein, L. Berglund, P.J. Havel, Consuming fructose-sweetened, not glucose-sweetened, beverages increases visceral adiposity and lipids and decreases insulin sensitivity in overweight/obese humans, J. Clin. Investig. 119 (2009) 1322-1334.

[163] K. Maeda, Y. Noguchi, T. Fukui, The effects of cessation from cigarette smoking on the lipid and lipoprotein profiles: a meta-analysis, Prev. Med. 37 (2003) 283-290.

[164] R.P. Mensink, P.L. Zock, A.D. Kester, M.B. Katan, Effects of dietary fatty acids and carbohydrates on the ratio of serum total to HDL cholesterol and on serum lipids and apolipoproteins: a meta-analysis of 60 controlled trials, Am. J. Clin. Nutr. 77 (2003) 1146-1155.

[168] K.M. Huffman, V.H. Hawk, S.T. Henes, C.I. Ocampo, M.C. Orenduff, C.A. Slentz, J.L. Johnson, J.A. Houmard, G.P. Samsa, W.E. Kraus, C.W. Bales, Exercise effects on lipids in persons with varying dietary patterns-does diet matter if they exercise? Responses in studies of a targeted risk reduction intervention through defined exercise I, Am. Heart J. 164 (2012) 117-124.

[169] W.E. Kraus, J.A. Houmard, B.D. Duscha, K.J. Knetzger, M.B. Wharton, J.S. McCartney, C.W. Bales, S. Henes, G.P. Samsa, J.D. Otvos, K.R. Kulkarni, C.A. Slentz, Effects of the amount and intensity of exercise on plasma lipoproteins, N. Engl. J. Med. 347 (2002) 1483-1492.

[170] S.E. Brien, P.E. Ronksley, B.J. Turner, K.J. Mukamal, W.A. Ghali, Effect of alcohol consumption on biological markers associated with risk of coronary heart disease: systematic review and meta-analysis of interventional studies, BMJ 342 (2011) d636.

[171] C. De Natale, G. Annuzzi, L. Bozzetto, R. Mazzarella, G. Costabile, O. Ciano, G. Riccardi, A.A. Rivellese, Effects of a plant-based high-carbohydrate/high-fiber diet versus high-monounsaturated fat/low-carbohydrate diet on postprandial lipids in type 2 diabetic patients, Diabetes Care 32 (2009) 2168-2173.

[172] S. Liu, J.E. Manson, M.J. Stampfer, M.D. Holmes, F.B. Hu, S.E. Hankinson, W.C. Willett, Dietary glycemic load assessed by food-frequency questionnaire in relation to plasma high-density-lipoprotein cholesterol and fasting plasma triacylglycerols in postmenopausal women, Am. J. Clin. Nutr. 73 (2001) 560-566.

[173] K.L. Stanhope, V. Medici, A.A. Bremer, V. Lee, H.D. Lam, M.V. Nunez, G.X. Chen, N.L. Keim, P.J. Havel, A dose-response study of consuming high-fructose corn syrup-sweetened beverages on lipid/lipoprotein risk factors for cardiovascular disease in young adults, Am. J. Clin. Nutr. 101 (2015) 1144-1154.

[174] M.R. Taskinen, S. Soderlund, L.H. Bogl, A. Hakkarainen, N. Matikainen, K.H. Pietilainen, S. Rasanen, N. Lundbom, E. Bjornson, B. Eliasson, R.M. Mancina, S. Romeo, N. Almeras, G.D. Pepa, C. Vetrani, A. Prinster, G. Annuzzi, A. Rivellese, J.P. Despres, J. Boren, Adverse effects of fructose on cardiometabolic risk factors and hepatic lipid metabolism in subjects with abdominal obesity, J. Intern. Med. 282 (2017) 187-201.

[175] Look Ahead Research Group, R.R. Wing, P. Bolin, F.L. Brancati, G.A. Bray, J.M. Clark, M. Coday, R.S. Crow, J.M. Curtis, C.M. Egan, M.A. Espeland, M. Evans, J.P. Foreyt, S. Ghazarian, E.W. Gregg, B. Harrison, H.P. Hazuda, J.O. Hill, E.S. Horton, V.S. Hubbard, J.M. Jakicic, R.W. Jeffery, K.C. Johnson, S.E. Kahn, A.E. Kitabchi, W.C. Knowler, C.E. Lewis, B.J. Maschak-Carey, M.G. Montez, A. Murillo, D.M. Nathan, J. Patricio, A. Peters, X. Pi-Sunyer, H. Pownall, D. Reboussin, J.G. Regensteiner, A.D. Rickman, D.H. Ryan, M. Safford, T.A. Wadden, L.E. Wagenknecht, D.S. West, D.F. Williamson, S.Z. Yanovski, Cardiovascular effects of intensive lifestyle intervention in type 2 diabetes, $\mathrm{N}$. Engl. J. Med. 369 (2013) 145-154.

[176] J.A. Batsis, L.E. Gill, R.K. Masutani, A.M. Adachi-Mejia, H.B. Blunt, P.J. Bagley, F. Lopez-Jimenez, S.J. Bartels, Weight loss interventions in older adults with obesity: a systematic review of randomized controlled trials since 2005, J. Am. Geriatr. Soc. 65 (2017) 257-268.

[177] W.S. Harris, D. Mozaffarian, E. Rimm, P. Kris-Etherton, L.L. Rudel, L.J. Appel, M.M. Engler, M.B. Engler, F. Sacks, Omega- 6 fatty acids and risk for cardiovascular disease: a science advisory from the American heart association nutrition subcommittee of the Council on nutrition, physical activity, and metabolism; Council on cardiovascular nursing; and Council on epidemiology and prevention, Circulation 119 (2009) 902-907.

[178] D. Mozaffarian, R.N. Lemaitre, I.B. King, X. Song, H. Huang, F.M. Sacks, E.B. Rimm, M. Wang, D.S. Siscovick, Plasma phospholipid long-chain omega-3 fatty acids and total and cause-specific mortality in older adults: a cohort study, Ann. Intern. Med. 158 (2013) 515-525.

[179] G. Riccardi, O. Vaccaro, G. Costabile, A.A. Rivellese, How well can we control dyslipidemias through lifestyle modifications? Curr. Cardiol. Rep. 18 (2016) 66.

[180] S.B. Seidelmann, B. Claggett, S. Cheng, M. Henglin, A. Shah, L.M. Steffen, A.R. Folsom, E.B. Rimm, W.C. Willett, S.D. Solomon, Dietary carbohydrate intake and mortality: a prospective cohort study and meta-analysis, Lancet Public Health 3 (2018) e419-e428.

[181] M. Dehghan, A. Mente, S. Rangarajan, P. Sheridan, V. Mohan, R. Iqbal, R. Gupta, S. Lear, E. Wentzel-Viljoen, A. Avezum, P. Lopez-Jaramillo, P. Mony, R.P. Varma,
R. Kumar, J. Chifamba, K.F. Alhabib, N. Mohammadifard, A. Oguz, F. Lanas, D. Rozanska, K.B. Bostrom, K. Yusoff, L.P. Tsolkile, A. Dans, A. Yusufali, A. Orlandini, P. Poirier, R. Khatib, B. Hu, L. Wei, L. Yin, A. Deeraili, K. Yeates, R. Yusuf, N. Ismail, D. Mozaffarian, K. Teo, S.S. Anand, S. Yusuf, Prospective Urban Rural Epidemiology study investigators. Association of dairy intake with cardiovascular disease and mortality in 21 countries from five continents (PURE): a prospective cohort study, Lancet 392 (2018) 2288-2297.

[182] A. Poli, F. Marangoni, R. Paoletti, E. Mannarino, G. Lupattelli, A. Notarbartolo, P. Aureli, F. Bernini, A. Cicero, A. Gaddi, A. Catapano, C. Cricelli, M. Gattone, W. Marrocco, M. Porrini, R. Stella, A. Vanotti, M. Volpe, R. Volpe, C. Cannella, A. Pinto, E. Del Toma, C. La Vecchia, A. Tavani, E. Manzato, G. Riccardi, C. Sirtori, A. Zambon, Nutrition Foundation of Italy. Non-pharmacological control of plasma cholesterol levels, Nutr. Metab. Cardiovasc. Dis. 18 (2008) S1-S16.

[183] Global Burden of Disease 2016 Alcohol Collaborators, Alcohol use and burden for 195 countries and territories, 1990-2016: a systematic analysis for the Global Burden of Disease Study 2016, Lancet 392 (2018) 1015-1035.

[184] A.M. Wood, S. Kaptoge, A.S. Butterworth, P. Willeit, S. Warnakula, T. Bolton, E. Paige, D.S. Paul, M. Sweeting, S. Burgess, S. Bell, W. Astle, D. Stevens, A. Koulman, R.M. Selmer, W.M.M. Verschuren, S. Sato, I. Njolstad, M. Woodward, V. Salomaa, B.G. Nordestgaard, B.B. Yeap, A. Fletcher, O. Melander, L.H. Kuller, B. Balkau, M. Marmot, W. Koenig, E. Casiglia, C. Cooper, V. Arndt, O.H. Franco, P. Wennberg, J. Gallacher, A.G. de la Camara, H. Volzke, C.C. Dahm, C.E. Dale, M.M. Bergmann, C.J. Crespo, Y.T. van der Schouw, R. Kaaks, L.A. Simons, P. Lagiou, J.D. Schoufour, J.M.A. Boer, T.J. Key, B. Rodriguez, C. Moreno-Iribas, K.W. Davidson, J.O. Taylor, C. Sacerdote, R.B. Wallace, J.R. Quiros, R. Tumino, D.G. Blazer II, A. Linneberg, M. Daimon, S. Panico, B. Howard, G. Skeie, T. Strandberg, E. Weiderpass, P.J. Nietert, B.M. Psaty, D. Kromhout, E. SalamancaFernandez, S. Kiechl, H.M. Krumholz, S. Grioni, D. Palli, J.M. Huerta, J. Price, J. Sundstrom, L. Arriola, H. Arima, R.C. Travis, D.B. Panagiotakos, A. Karakatsani, A. Trichopoulou, T. Kuhn, D.E. Grobbee, E. Barrett-Connor, N. van Schoor, H. Boeing, K. Overvad, J. Kauhanen, N. Wareham, C. Langenberg, N. Forouhi, M. Wennberg, J.P. Despres, M. Cushman, J.A. Cooper, C.J. Rodriguez, M. Sakurai, J.E. Shaw, M. Knuiman, T. Voortman, C. Meisinger, A. Tjonneland, H. Brenner, L. Palmieri, J. Dallongeville, E.J. Brunner, G. Assmann, M. Trevisan, R.F. Gillum, I. Ford, N. Sattar, M. Lazo, S.G. Thompson, P. Ferrari, D.A. Leon, G.D. Smith, R. Peto, R. Jackson, E. Banks, E. Di Angelantonio, J. Danesh, Emerging Risk Factors Collaboration/EPIC-CVD/UK Biobank Alcohol Study Group. Risk thresholds for alcohol consumption: combined analysis of individual-participant data for 599912 current drinkers in 83 prospective studies, Lancet 391 (2018) 1513-1523.

[185] G.G. De Backer, Food supplements with red yeast rice: more regulations are needed, Eur J Prev Cardiol 24 (2017) 1429-1430.

[186] L. Hartley, M.D. May, E. Loveman, J.L. Colquitt, K. Rees, Dietary fibre for the primary prevention of cardiovascular disease, Cochrane Database Syst. Rev. 1 (2016) CD011472.

[187] M. Pirro, C. Vetrani, C. Bianchi, M.R. Mannarino, F. Bernini, A.A. Rivellese, Joint position statement on "Nutraceuticals for the treatment of hypercholesterolemia" of the Italian society of diabetology (SID) and of the Italian society for the study of arteriosclerosis (SISA), Nutr. Metab. Cardiovasc. Dis. 27 (2017) 2-17.

[188] A. Dewell, P.L. Hollenbeck, C.B. Hollenbeck, Clinical review: a critical evaluation of the role of soy protein and isoflavone supplementation in the control of plasma cholesterol concentrations, J. Clin. Endocrinol. Metab. 91 (2006) 772-780.

[189] R. Mas, G. Castano, J. Illnait, L. Fernandez, J. Fernandez, C. Aleman, V. Pontigas, M. Lescay, Effects of policosanol in patients with type II hypercholesterolemia and additional coronary risk factors, Clin. Pharmacol. Ther. 65 (1999) 439-447.

[190] Z. Reiner, E. Tedeschi-Reiner, Z. Romic, Effects of rice policosanol on serum lipoproteins, homocysteine, fibrinogen and C-reactive protein in hypercholesterolaemic patients, Clin. Drug Investig. 25 (2005) 701-707.

[191] J. Lan, Y. Zhao, F. Dong, Z. Yan, W. Zheng, J. Fan, G. Sun, Meta-analysis of the effect and safety of berberine in the treatment of type 2 diabetes mellitus, hyperlipemia and hypertension, J. Ethnopharmacol. 161 (2015) 69-81.

[192] F.M. Sacks, A.H. Lichtenstein, J.H.Y. Wu, L.J. Appel, M.A. Creager, P.M. KrisEtherton, M. Miller, E.B. Rimm, L.L. Rudel, J.G. Robinson, N.J. Stone, L.V. Van Horn, American Heart Association. Dietary fats and cardiovascular disease: a presidential advisory from the American Heart Association, Circulation 136 (2017) e1-e23.

[193] T.A. Jacobson, S.B. Glickstein, J.D. Rowe, P.N. Soni, Effects of eicosapentaenoic acid and docosahexaenoic acid on low-density lipoprotein cholesterol and other lipids: a review, J Clin Lipidol 6 (2012) 5-18.

[194] D.L. Bhatt, P.G. Steg, M. Miller, E.A. Brinton, T.A. Jacobson, S.B. Ketchum, R.T. Doyle Jr., R.A. Juliano, L. Jiao, C. Granowitz, J.C. Tardif, C.M. Ballantyne, REDUCE-IT Investigators, Cardiovascular risk reduction with icosapent ethyl for hypertriglyceridemia, N. Engl. J. Med. 380 (2019) 11-22.

[195] D.I. Chasman, F. Giulianini, J. MacFadyen, B.J. Barratt, F. Nyberg, P.M. Ridker, Genetic determinants of statin-induced low-density lipoprotein cholesterol reduction: the justification for the use of statins in prevention: an intervention trial evaluating rosuvastatin (JUPITER) trial, Circ Cardiovasc Genet 5 (2012) 257-264.

[196] Z. Reiner, Resistance and intolerance to statins, Nutr. Metab. Cardiovasc. Dis. 24 (2014) 1057-1066.

[197] P.M. Moriarty, P.D. Thompson, C.P. Cannon, J.R. Guyton, J. Bergeron, F.J. Zieve, E. Bruckert, T.A. Jacobson, S.L. Kopecky, M.T. Baccara-Dinet, Y. Du, R. Pordy, D.A. Gipe, ODYSSEY ALTERNATIVE Investigators. Efficacy and safety of alirocumab vs ezetimibe in statin-intolerant patients, with a statin rechallenge arm: the ODYSSEY ALTERNATIVE randomized trial, J Clin Lipidol 9 (2015) 758-769.

[198] E. Stroes, D. Colquhoun, D. Sullivan, F. Civeira, R.S. Rosenson, G.F. Watts, E. Bruckert, L. Cho, R. Dent, B. Knusel, A. Xue, R. Scott, S.M. Wasserman, M. Rocco, GAUSS-2 Investigators, Anti-PCSK9 antibody effectively lowers 
cholesterol in patients with statin intolerance: the GAUSS-2 randomized, placebocontrolled phase 3 clinical trial of evolocumab, J. Am. Coll. Cardiol. 63 (2014) 2541-2548.

[199] Z. Reiner, Managing the residual cardiovascular disease risk associated with HDLcholesterol and triglycerides in statin-treated patients: a clinical update, Nutr. Metab. Cardiovasc. Dis. 23 (2013) 799-807.

[200] A. Sharma, P.H. Joshi, S. Rinehart, K.M. Thakker, A. Lele, S. Voros, Baseline very low-density lipoprotein cholesterol is associated with the magnitude of triglyceride lowering on statins, fenofibric acid, or their combination in patients with mixed dyslipidemia, J Cardiovasc Transl Res 7 (2014) 465-474.

[201] P.J. Barter, G. Brandrup-Wognsen, M.K. Palmer, S.J. Nicholls, Effect of statins on HDL-C: a complex process unrelated to changes in LDL-C: analysis of the VOYAGER Database, J. Lipid Res. 51 (2010) 1546-1553.

[202] S. Tsimikas, J.L. Witztum, E.R. Miller, W.J. Sasiela, M. Szarek, A.G. Olsson, G.G. Schwartz, Myocardial Ischemia Reduction with Aggressive Cholesterol Lowering (MIRACL) Study Investigators. High-dose atorvastatin reduces total plasma levels of oxidized phospholipids and immune complexes present on apolipoprotein B-100 in patients with acute coronary syndromes in the MIRACL trial, Circulation 110 (2004) 1406-1412.

[203] A.V. Khera, B.M. Everett, M.P. Caulfield, F.M. Hantash, J. Wohlgemuth, P.M. Ridker, S. Mora, Lipoprotein(a) concentrations, rosuvastatin therapy, and residual vascular risk: an analysis from the JUPITER trial (justification for the use of statins in prevention: an intervention trial evaluating rosuvastatin), Circulation 129 (2014) 635-642.

[204] J. Davignon, Beneficial cardiovascular pleiotropic effects of statins, Circulation 109 (2004) III39-III43.

[205] A. Oesterle, U. Laufs, J.K. Liao, Pleiotropic effects of statins on the cardiovascular system, Circ. Res. 120 (2017) 229-243.

[206] T.R. Pedersen, Pleiotropic effects of statins: evidence against benefits beyond LDLcholesterol lowering, Am. J. Cardiovasc. Drugs 10 (2010) 10-17.

[207] B. Genser, W. Marz, Low density lipoprotein cholesterol, statins and cardiovascular events: a meta-analysis, Clin. Res. Cardiol. 95 (2006) 393-404.

[208] A.L. Gould, J.E. Rossouw, N.C. Santanello, J.F. Heyse, C.D. Furberg, Cholesterol reduction yields clinical benefit. A new look at old data, Circulation 91 (1995) 2274-2282.

[209] A.L. Gould, J.E. Rossouw, N.C. Santanello, J.F. Heyse, C.D. Furberg, Cholesterol reduction yields clinical benefit: impact of statin trials, Circulation 97 (1998) 946-952.

[210] J.C. LaRosa, J. He, S. Vupputuri, Effect of statins on risk of coronary disease: a meta-analysis of randomized controlled trials, J. Am. Med. Assoc. 282 (1999) 2340-2346.

[211] E.J. Mills, P. Wu, G. Chong, I. Ghement, S. Singh, E.A. Akl, O. Eyawo, G. Guyatt, O. Berwanger, M. Briel, Efficacy and safety of statin treatment for cardiovascular disease: a network meta-analysis of 170,255 patients from 76 randomized trials, QJM 104 (2011) 109-124.

[212] K.K. Ray, S.R. Seshasai, S. Erqou, P. Sever, J.W. Jukema, I. Ford, N. Sattar, Statins and all-cause mortality in high-risk primary prevention: a meta-analysis of 11 randomized controlled trials involving 65,229 participants, Arch. Intern. Med. 170 (2010) 1024-1031.

[213] F. Taylor, M.D. Huffman, A.F. Macedo, T.H. Moore, M. Burke, G. Davey Smith, K. Ward, S. Ebrahim, Statins for the primary prevention of cardiovascular disease, Cochrane Database Syst. Rev. 1 (2013) CD004816.

[214] Cholesterol Treatment Trialists Collaboration, W.G. Herrington, J. Emberson, B. Mihaylova, L. Blackwell, C. Reith, M.D. Solbu, P.B. Mark, B. Fellstrom, A.G. Jardine, C. Wanner, H. Holdaas, J. Fulcher, R. Haynes, M.J. Landray, A. Keech, J. Simes, R. Collins, C. Baigent, Impact of renal function on the effects of LDL cholesterol lowering with statin-based regimens: a meta-analysis of individual participant data from 28 randomised trials, Lancet Diabetes Endocrinol 4 (2016) 829-839.

[215] H. Naci, J.J. Brugts, R. Fleurence, B. Tsoi, H. Toor, A.E. Ades, Comparative benefits of statins in the primary and secondary prevention of major coronary events and all-cause mortality: a network meta-analysis of placebo-controlled and activecomparator trials, Eur J Prev Cardiol 20 (2013) 641-657.

[216] I. Ford, H. Murray, C. McCowan, C.J. Packard, Long-term safety and efficacy of lowering low-density lipoprotein cholesterol with statin therapy: 20-year followup of West of Scotland Coronary Prevention Study, Circulation 133 (2016) 1073-1080.

[217] C. Cholesterol Treatment Trialists, Efficacy and safety of statin therapy in older people: a meta-analysis of individual participant data from 28 randomised controlled trials, Lancet 393 (2019) 407-415.

[218] J.K. Rogers, P.S. Jhund, A.C. Perez, M. Bohm, J.G. Cleland, L. Gullestad, J. Kjekshus, D.J. van Veldhuisen, J. Wikstrand, H. Wedel, J.J. McMurray, S.J. Pocock, Effect of rosuvastatin on repeat heart failure hospitalizations: the CORONA trial (controlled rosuvastatin multinational trial in heart failure), JACC Heart Fail 2 (2014) 289-297.

[219] L. Tavazzi, A.P. Maggioni, R. Marchioli, S. Barlera, M.G. Franzosi, R. Latini, D. Lucci, G.L. Nicolosi, M. Porcu, G. Tognoni, Gissi-HF. Investigator, Effect of rosuvastatin in patients with chronic heart failure (the GISSI-HF trial): a randomised, double-blind, placebo-controlled trial, Lancet 372 (2008) 1231-1239.

[220] C. Wanner, V. Krane, W. Marz, M. Olschewski, J.F. Mann, G. Ruf, E. Ritz, German Diabetes and Dialysis Study Investigators. Atorvastatin in patients with type 2 diabetes mellitus undergoing hemodialysis, N. Engl. J. Med. 353 (2005) 238-248.

[221] B.C. Fellström, A.G. Jardine, R.E. Schmieder, H. Holdaas, K. Bannister, J. Beutler, D.W. Chae, A. Chevaile, S.M. Cobbe, C. Gronhagen-Riska, J.J. De Lima, R. Lins, G. Mayer, A.W. McMahon, H.H. Parving, G. Remuzzi, O. Samuelsson, S. Sonkodi, D. Sci, G. Süleymanlar, D. Tsakiris, V. Tesar, V. Todorov, A. Wiecek,
R.P. Wüthrich, M. Gottlow, E. Johnsson, F. Zannad, AURORA Study Group, Rosuvastatin and cardiovascular events in patients undergoing hemodialysis, $\mathrm{N}$. Engl. J. Med. 360 (2009) 1395-1407.

[222] C. Baigent, M.J. Landray, C. Reith, J. Emberson, D.C. Wheeler, C. Tomson, C. Wanner, V. Krane, A. Cass, J. Craig, B. Neal, L. Jiang, L.S. Hooi, A. Levin, L. Agodoa, M. Gaziano, B. Kasiske, R. Walker, Z.A. Massy, B. Feldt-Rasmussen, U. Krairittichai, V. Ophascharoensuk, B. Fellstrom, H. Holdaas, V. Tesar, A. Wiecek, D. Grobbee, D. de Zeeuw, C. Gronhagen-Riska, T. Dasgupta, D. Lewis, W. Herrington, M. Mafham, W. Majoni, K. Wallendszus, R. Grimm, T. Pedersen, J. Tobert, J. Armitage, A. Baxter, C. Bray, Y. Chen, Z. Chen, M. Hill, C. Knott, S. Parish, D. Simpson, P. Sleight, A. Young, R. Collins, SHARP Investigators, The effects of lowering LDL cholesterol with simvastatin plus ezetimibe in patients with chronic kidney disease (Study of Heart and Renal Protection): a randomised placebo-controlled trial, Lancet 377 (2011) 2181-2192.

[223] Cholesterol Treatment Trialists Collaboration, J.R. Emberson, P.M. Kearney, L. Blackwell, C. Newman, C. Reith, N. Bhala, L. Holland, R. Peto, A. Keech, R. Collins, J. Simes, C. Baigent, Lack of effect of lowering LDL cholesterol on cancer: meta-analysis of individual data from 175,000 people in 27 randomised trials of statin therapy, PLoS One 7 (2012) e29849.

[224] B. McGuinness, D. Craig, R. Bullock, R. Malouf, P. Passmore, Statins for the treatment of dementia, Cochrane Database Syst. Rev. 7 (2014) CD007514.

[225] L. Eslami, S. Merat, R. Malekzadeh, S. Nasseri-Moghaddam, H. Aramin, Statins for non-alcoholic fatty liver disease and non-alcoholic steatohepatitis, Cochrane Database Syst. Rev. 12 (2013) CD008623.

[226] K. Rahimi, N. Bhala, P. Kamphuisen, J. Emberson, S. Biere-Rafi, V. Krane, M. Robertson, J. Wikstrand, J. McMurray, Effect of statins on venous thromboembolic events: a meta-analysis of published and unpublished evidence from randomised controlled trials, PLoS Med. 9 (2012) e1001310, , https://doi.org/10. 1371/journal.pmed.1001310 Epub 18 September 2012.

[227] K. Rahimi, J. Emberson, P. McGale, W. Majoni, A. Merhi, F.W. Asselbergs, V. Krane, P.W. Macfarlane, PROSPER Executive, Effect of statins on atrial fibrillation: collaborative meta-analysis of published and unpublished evidence from randomised controlled trials, BMJ 342 (2011) d1250.

[228] Z. Zheng, R. Jayaram, L. Jiang, J. Emberson, Y. Zhao, Q. Li, J. Du, S. Guarguagli, M. Hill, Z. Chen, R. Collins, B. Casadei, Perioperative rosuvastatin in cardiac surgery, N. Engl. J. Med. 374 (2016) 1744-1753.

[229] A.D. Raval, T. Hunter, B. Stuckey, R.J. Hart, Statins for women with polycystic ovary syndrome not actively trying to conceive, Cochrane Database Syst. Rev. 10 (2011) CD008565.

[230] B. McGuinness, D. Craig, R. Bullock, P. Passmore, Statins for the prevention of dementia, Cochrane Database Syst. Rev. 1 (2016) CD003160.

[231] R.P. Giugliano, F. Mach, K. Zavitz, C. Kurtz, K. Im, E. Kanevsky, J. Schneider, H. Wang, A. Keech, T.R. Pedersen, M.S. Sabatine, P.S. Sever, J.G. Robinson, N. Honarpour, S.M. Wasserman, B.R. Ott, EBBINGHAUS Investigators, Cognitive function in a randomized trial of evolocumab, N. Engl. J. Med. 377 (2017) 633-643.

[232] F. Mach, K.K. Ray, O. Wiklund, A. Corsini, A.L. Catapano, E. Bruckert, G. De Backer, R.A. Hegele, G.K. Hovingh, T.A. Jacobson, R.M. Krauss, U. Laufs, L.A. Leiter, W. Marz, B.G. Nordestgaard, F.J. Raal, M. Roden, R.D. Santos, E.A. Stein, E.S. Stroes, P.D. Thompson, L. Tokgozoglu, G.D. Vladutiu, B. Gencer, J.K. Stock, H.N. Ginsberg, M.J. Chapman, European Atherosclerosis Society Consensus Panel. Adverse effects of statin therapy: perception vs. the evidence focus on glucose homeostasis, cognitive, renal and hepatic function, haemorrhagic stroke and cataract, Eur. Heart J. 39 (2018) 2526-2539.

[233] R. Collins, C. Reith, J. Emberson, J. Armitage, C. Baigent, L. Blackwell, R. Blumenthal, J. Danesh, G.D. Smith, D. DeMets, S. Evans, M. Law, S. MacMahon, S. Martin, B. Neal, N. Poulter, D. Preiss, P. Ridker, I. Roberts, A. Rodgers, P. Sandercock, K. Schulz, P. Sever, J. Simes, L. Smeeth, N. Wald, S. Yusuf, R. Peto, Interpretation of the evidence for the efficacy and safety of statin therapy, Lancet 388 (2016) 2532-2561.

[234] E.S. Stroes, P.D. Thompson, A. Corsini, G.D. Vladutiu, F.J. Raal, K.K. Ray, M. Roden, E. Stein, L. Tokgozoglu, B.G. Nordestgaard, E. Bruckert, G. De Backer, R.M. Krauss, U. Laufs, R.D. Santos, R.A. Hegele, G.K. Hovingh, L.A. Leiter, F. Mach, W. Marz, C.B. Newman, O. Wiklund, T.A. Jacobson, A.L. Catapano, M.J. Chapman, H.N. Ginsberg, European atherosclerosis society consensus panel. Statin-associated muscle symptoms: impact on statin therapy-European atherosclerosis society consensus panel statement on assessment, aetiology and management, Eur. Heart J. 36 (2015) 1012-1022.

[235] M. Law, A.R. Rudnicka, Statin safety: a systematic review, Am. J. Cardiol. 97 (2006) 52C-60C.

[236] E. Bruckert, G. Hayem, S. Dejager, C. Yau, B. Begaud, Mild to moderate muscular symptoms with high-dosage statin therapy in hyperlipidemic patients-the PRIMO study, Cardiovasc. Drugs Ther. 19 (2005) 403-414.

[237] M.H. Davidson, J.A. Clark, L.M. Glass, A. Kanumalla, Statin safety: an appraisal from the adverse event reporting system, Am. J. Cardiol. 97 (2006) 32C-43C.

[238] A. Gupta, D. Thompson, A. Whitehouse, T. Collier, B. Dahlof, N. Poulter, R. Collins, P. Sever, A.S.C.O.T. Investigators, Adverse events associated with unblinded, but not with blinded, statin therapy in the Anglo-Scandinavian Cardiac Outcomes Trial-Lipid-Lowering Arm (ASCOT-LLA): a randomised double-blind placebo-controlled trial and its non-randomised non-blind extension phase, Lancet 389 (2017) 2473-2481.

[239] J.A. Finegold, C.H. Manisty, B. Goldacre, A.J. Barron, D.P. Francis, What proportion of symptomatic side effects in patients taking statins are genuinely caused by the drug? Systematic review of randomized placebo-controlled trials to aid individual patient choice, Eur J Prev Cardiol 21 (2014) 464-474.

[240] H. Naci, J. Brugts, T. Ades, Comparative tolerability and harms of individual 
statins: a study-level network meta-analysis of 246955 participants from 135 randomized, controlled trials, Circ Cardiovasc Qual Outcomes 6 (2013) 390-399.

[241] P.M. Moriarty, T.A. Jacobson, E. Bruckert, P.D. Thompson, J.R. Guyton, M.T. Baccara-Dinet, D. Gipe, Efficacy and safety of alirocumab, a monoclonal antibody to PCSK9, in statin-intolerant patients: design and rationale of ODYSSEY ALTERNATIVE, a randomized phase 3 trial, J Clin Lipidol 8 (2014) 554-561.

[242] A.J. Keating, K.B. Campbell, J.R. Guyton, Intermittent nondaily dosing strategies in patients with previous statin-induced myopathy, Ann. Pharmacother. 47 (2013) 398-404.

[243] Z.A. Marcum, J.P. Vande Griend, S.A. Linnebur, FDA drug safety communications: a narrative review and clinical considerations for older adults, Am. J. Geriatr. Pharmacother. 10 (2012) 264-271.

[244] N. Chalasani, H. Aljadhey, J. Kesterson, M.D. Murray, S.D. Hall, Patients with elevated liver enzymes are not at higher risk for statin hepatotoxicity, Gastroenterology 126 (2004) 1287-1292.

[245] P. Dongiovanni, S. Petta, V. Mannisto, R.M. Mancina, R. Pipitone, V. Karja, M. Maggioni, P. Kakela, O. Wiklund, E. Mozzi, S. Grimaudo, D. Kaminska, R. Rametta, A. Craxi, S. Fargion, V. Nobili, S. Romeo, J. Pihlajamaki, L. Valenti, Statin use and non-alcoholic steatohepatitis in at risk individuals, J. Hepatol. 63 (2015) 705-712.

[246] R. Vuppalanchi, E. Teal, N. Chalasani, Patients with elevated baseline liver enzymes do not have higher frequency of hepatotoxicity from lovastatin than those with normal baseline liver enzymes, Am. J. Med. Sci. 329 (2005) 62-65.

[247] N. Sattar, D. Preiss, H.M. Murray, P. Welsh, B.M. Buckley, A.J. de Craen, S.R. Seshasai, J.J. McMurray, D.J. Freeman, J.W. Jukema, P.W. Macfarlane, C.J. Packard, D.J. Stott, R.G. Westendorp, J. Shepherd, B.R. Davis, S.L. Pressel, R. Marchioli, R.M. Marfisi, A.P. Maggioni, L. Tavazzi, G. Tognoni, J. Kjekshus, T.R. Pedersen, T.J. Cook, A.M. Gotto, M.B. Clearfield, J.R. Downs, H. Nakamura, Y. Ohashi, K. Mizuno, K.K. Ray, I. Ford, Statins and risk of incident diabetes: a collaborative meta-analysis of randomised statin trials, Lancet 375 (2010) 735-742.

[248] D. Preiss, S.R. Seshasai, P. Welsh, S.A. Murphy, J.E. Ho, D.D. Waters D.A. DeMicco, P. Barter, C.P. Cannon, M.S. Sabatine, E. Braunwald, J.J. Kastelein, J.A. de Lemos, M.A. Blazing, T.R. Pedersen, M.J. Tikkanen, N. Sattar, K.K. Ray, Risk of incident diabetes with intensive-dose compared with moderate-dose statin therapy: a meta-analysis, J. Am. Med. Assoc. 305 (2011) 2556-2564.

[249] D.D. Waters, J.E. Ho, S.M. Boekholdt, D.A. DeMicco, J.J. Kastelein, M. Messig, A. Breazna, T.R. Pedersen, Cardiovascular event reduction versus new-onset diabetes during atorvastatin therapy: effect of baseline risk factors for diabetes, J. Am. Coll. Cardiol. 61 (2013) 148-152.

[250] D.I. Swerdlow, D. Preiss, K.B. Kuchenbaecker, M.V. Holmes, J.E. Engmann, T. Shah, R. Sofat, S. Stender, P.C. Johnson, R.A. Scott, M. Leusink, N. Verweij, S.J. Sharp, Y. Guo, C. Giambartolomei, C. Chung, A. Peasey, A. Amuzu, K. Li, J. Palmen, P. Howard, J.A. Cooper, F. Drenos, Y.R. Li, G. Lowe, J. Gallacher, M.C. Stewart, I. Tzoulaki, S.G. Buxbaum, A.D. van der, N.G. Forouhi, N.C. OnlandMoret, Y.T. van der Schouw, R.B. Schnabel, J.A. Hubacek, Kubinova R, M. Baceviciene, A. Tamosiunas, A. Pajak, R. Topor-Madry, U. Stepaniak, S. Malyutina, D. Baldassarre, B. Sennblad, E. Tremoli, U. de Faire, F. Veglia, I. Ford, J.W. Jukema, R.G. Westendorp, G.J. de Borst, P.A. de Jong, A. Algra, W. Spiering, A.H. Maitland-van der Zee, O.H. Klungel, A. de Boer, P.A. Doevendans, C.B. Eaton, J.G. Robinson, D. Duggan, D. Consortium, M. Consortium, C. InterAct, J. Kjekshus, J.R. Downs, A.M. Gotto, A.C. Keech, R. Marchioli, G. Tognoni, P.S. Sever, N.R. Poulter, D.D. Waters, T.R. Pedersen, P. Amarenco, H. Nakamura, J.J. McMurray, J.D. Lewsey, D.I. Chasman, P.M. Ridker, A.P. Maggioni, L. Tavazzi, K.K. Ray, S.R. Seshasai, J.E. Manson, J.F. Price, P.H. Whincup, R.W. Morris, D.A. Lawlor, G.D. Smith, Y. Ben-Shlomo, P.J. Schreiner, M. Fornage, D.S. Siscovick, M. Cushman, M. Kumari, N.J. Wareham, W.M. Verschuren, S. Redline, S.R. Patel, J.C. Whittaker, A. Hamsten, J.A. Delaney, C. Dale, T.R. Gaunt, A. Wong, D. Kuh, R. Hardy, S. Kathiresan, B.A. Castillo, P. van der Harst, E.J. Brunner, A. Tybjaerg-Hansen, M.G. Marmot, R.M. Krauss, M. Tsai, J. Coresh, R.C. Hoogeveen, B.M. Psaty, L.A. Lange, H. Hakonarson, F. Dudbridge, S.E. Humphries, P.J. Talmud, M. Kivimaki, N.J. Timpson, C. Langenberg, F.W. Asselbergs, M. Voevoda, M. Bobak, H. Pikhart, J.G. Wilson, A.P. Reiner, B.J. Keating, A.D. Hingorani, N. Sattar, HMG-coenzyme A reductase inhibition, type 2 diabetes, and bodyweight: evidence from genetic analysis and randomised trials, Lancet 385 (2015) 351-361.

[251] J.S. McKinney, W.J. Kostis, Statin therapy and the risk of intracerebral hemorrhage: a meta-analysis of 31 randomized controlled trials, Stroke 43 (2012) 2149-2156.

[252] D.G. Hackam, M. Woodward, L.K. Newby, D.L. Bhatt, M. Shao, E.E. Smith, A. Donner, M. Mamdani, J.D. Douketis, H. Arima, J. Chalmers, S. MacMahon, D.L. Tirschwell, B.M. Psaty, C.D. Bushnell, M.I. Aguilar, D.J. Capampangan, D.J. Werring, P. De Rango, A. Viswanathan, N. Danchin, C.L. Cheng, Y.H. Yang, B.M. Verdel, M.S. Lai, J. Kennedy, S. Uchiyama, T. Yamaguchi, Y. Ikeda, M. Mrkobrada, Statins and intracerebral hemorrhage: collaborative systematic review and meta-analysis, Circulation 124 (2011) 2233-2242.

[253] S.C. Palmer, S.D. Navaneethan, J.C. Craig, V. Perkovic, D.W. Johnson, S.U. Nigwekar, J. Hegbrant, G.F. Strippoli, HMG CoA reductase inhibitors (statins) for kidney transplant recipients, Cochrane Database Syst. Rev. 1 (2014) CD005019.

[254] R. Agarwal, Effects of statins on renal function, Am. J. Cardiol. 97 (2006) 748-755.

[255] J.E. Sidaway, R.G. Davidson, F. McTaggart, T.C. Orton, R.C. Scott, G.J. Smith, N.J. Brunskill, Inhibitors of 3-hydroxy-3-methylglutaryl-CoA reductase reduce receptor-mediated endocytosis in opossum kidney cells, J. Am. Soc. Nephrol. 15
(2004) 2258-2265.

[256] M.H. Davidson, Rosuvastatin safety: lessons from the FDA review and post-approval surveillance, Expert Opin. Drug Saf. 3 (2004) 547-557.

[257] A. Egan, E. Colman, Weighing the benefits of high-dose simvastatin against the risk of myopathy, N. Engl. J. Med. 365 (2011) 285-287.

[258] O. Wiklund, C. Pirazzi, S. Romeo, Monitoring of lipids, enzymes, and creatine kinase in patients on lipid-lowering drug therapy, Curr. Cardiol. Rep. 15 (2013) 397.

[259] R. Franssen, M. Vergeer, E.S. Stroes, J.J. Kastelein, Combination statin-fibrate therapy: safety aspects, Diabetes Obes. Metab. 11 (2009) 89-94.

[260] N. Holoshitz, A.A. Alsheikh-Ali, R.H. Karas, Relative safety of gemfibrozil and fenofibrate in the absence of concomitant cerivastatin use, Am. J. Cardiol. 101 (2008) 95-97.

[261] B.A. Phan, T.D. Dayspring, P.P. Toth, Ezetimibe therapy: mechanism of action and clinical update, Vasc. Health Risk Manag. 8 (2012) 415-427.

[262] A. Pandor, R.M. Ara, I. Tumur, A.J. Wilkinson, S. Paisley, A. Duenas, P.N. Durrington, J. Chilcott, Ezetimibe monotherapy for cholesterol lowering in 2,722 people: systematic review and meta-analysis of randomized controlled trials, J. Intern. Med. 265 (2009) 568-580.

[263] D. Morrone, W.S. Weintraub, P.P. Toth, M.E. Hanson, R.S. Lowe, J. Lin, A.K. Shah, A.M. Tershakovec, Lipid-altering efficacy of ezetimibe plus statin and statin monotherapy and identification of factors associated with treatment response: a pooled analysis of over 21,000 subjects from 27 clinical trials, Atherosclerosis 223 (2012) 251-261.

[264] M.R. Jones, O.M. Nwose, Role of colesevelam in combination lipid-lowering therapy, Am. J. Cardiovasc. Drugs 13 (2013) 315-323.

[265] S.E. Nissen, E. Stroes, R.E. Dent-Acosta, R.S. Rosenson, S.J. Lehman, N. Sattar, D. Preiss, E. Bruckert, R. Ceska, N. Lepor, C.M. Ballantyne, I. Gouni-Berthold, M. Elliott, D.M. Brennan, S.M. Wasserman, R. Somaratne, R. Scott, E.A. Stein, Gauss-3 Investigators, Efficacy and tolerability of evolocumab vs ezetimibe in patients with muscle-related statin intolerance: the GAUSS-3 randomized clinical trial, J. Am. Med. Assoc. 315 (2016) 1580-1590.

[266] A.B. Rossebø, T.R. Pedersen, K. Boman, P. Brudi, J.B. Chambers, K. Egstrup, E. Gerdts, C. Gohlke-Bärwolf, I. Holme, Y.A. Kesäniemi, W. Malbecq, C.A. Nienaber, S. Ray, T. Skjaerpe, K. Wachtell, R. Willenheimer, SEAS Investigators. Intensive lipid lowering with simvastatin and ezetimibe in aortic stenosis, N. Engl. J. Med. 359 (2008) 1343-1356.

[267] Sharp Collaborative Group, Study of Heart and Renal Protection (SHARP): randomized trial to assess the effects of lowering low-density lipoprotein cholesterol among 9,438 patients with chronic kidney disease, Am. Heart J. 160 (2010) 785-794 e10.

[268] B.A. Ference, C.P. Cannon, U. Landmesser, T.F. Luscher, A.L. Catapano, K.K. Ray, Reduction of low density lipoprotein-cholesterol and cardiovascular events with proprotein convertase subtilisin-kexin type 9 (PCSK9) inhibitors and statins: an analysis of FOURIER, SPIRE, and the Cholesterol Treatment Trialists Collaboration, Eur. Heart J. 39 (2018) 2540-2545.

[269] Myocardial Infarction Genetics Consortium Investigators, N.O. Stitziel, H.H. Won, A.C. Morrison, G.M. Peloso, R. Do, L.A. Lange, P. Fontanillas, N. Gupta, S. Duga, A. Goel, M. Farrall, D. Saleheen, P. Ferrario, I. Konig, R. Asselta, P.A. Merlini, N. Marziliano, M.F. Notarangelo, U. Schick, P. Auer, T.L. Assimes, M. Reilly, R. Wilensky, D.J. Rader, G.K. Hovingh, T. Meitinger, T. Kessler, A. Kastrati, K.L. Laugwitz, D. Siscovick, J.I. Rotter, S.L. Hazen, R. Tracy, S. Cresci, J. Spertus, R. Jackson, S.M. Schwartz, P. Natarajan, J. Crosby, D. Muzny, C. Ballantyne, S.S. Rich, C.J. O'Donnell, G. Abecasis, S. Sunyaev, D.A. Nickerson, J.E. Buring, P.M. Ridker, D.I. Chasman, E. Austin, Z. Ye, I.J. Kullo, P.E. Weeke, C.M. Shaffer, L.A. Bastarache, J.C. Denny, D.M. Roden, C. Palmer, P. Deloukas, D.Y. Lin, Z.Z. Tang, J. Erdmann, H. Schunkert, J. Danesh, J. Marrugat, R. Elosua, D. Ardissino, R. McPherson, H. Watkins, A.P. Reiner, J.G. Wilson, D. Altshuler, R.A. Gibbs, E.S. Lander, E. Boerwinkle, S. Gabriel, S. Kathiresan, Inactivating mutations in NPC1L1 and protection from coronary heart disease, N. Engl. J. Med. 371 (2014) 2072-2082.

[270] A. Pirillo, A.L. Catapano, G.D. Norata, Niemann-Pick C1-Like 1 (NPC1L1) inhibition and cardiovascular diseases, Curr. Med. Chem. 23 (2016) 983-999.

[271] K. Tsujita, S. Sugiyama, H. Sumida, H. Shimomura, T. Yamashita, K. Yamanaga, N. Komura, K. Sakamoto, H. Oka, K. Nakao, S. Nakamura, M. Ishihara, K. Matsui, N. Sakaino, N. Nakamura, N. Yamamoto, S. Koide, T. Matsumura, K. Fujimoto, R. Tsunoda, Y. Morikami, K. Matsuyama, S. Oshima, K. Kaikita, S. Hokimoto, H. Ogawa, PRECISE-IVUS Investigators. Impact of dual lipid-lowering strategy with ezetimibe and atorvastatin on coronary plaque regression in patients with percutaneous coronary intervention: the multicenter randomized controlled PRECISE-IVUS trial, J. Am. Coll. Cardiol. 66 (2015) 495-507.

[272] S.U. Khan, S. Talluri, H. Riaz, H. Rahman, F. Nasir, I. Bin Riaz, S. Sattur, H. Ahmed, E. Kaluski, R. Krasuski, A Bayesian network meta-analysis of PCSK9 inhibitors, statins and ezetimibe with or without statins for cardiovascular outcomes, Eur J Prev Cardiol 25 (2018) 844-853.

[273] K.C. Koskinas, G.C.M. Siontis, R. Piccolo, D. Mavridis, L. Raber, F. Mach, S. Windecker, Effect of statins and non-statin LDL-lowering medications on car diovascular outcomes in secondary prevention: a meta-analysis of randomized trials, Eur. Heart J. 39 (2018) 1172-1180.

[274] M. Mazidi, P. Rezaie, E. Karimi, A.P. Kengne, The effects of bile acid sequestrants on lipid profile and blood glucose concentrations: a systematic review and metaanalysis of randomized controlled trials, Int. J. Cardiol. 227 (2017) 850-857.

[275] C.P. Ooi, S.C. Loke, Colesevelam for type 2 diabetes mellitus: an abridged Cochrane review, Diabet. Med. 31 (2014) 2-14.

[276] The Lipid Research Clinics Program, Pre-entry characteristics of participants in the lipid research clinics' coronary primary prevention trial, J. Chronic Dis. 36 (1983) 
467-479.

[277] The Lipid Research Clinics Program, The lipid research clinics coronary primary prevention trial results. I. Reduction in incidence of coronary heart disease, J. Am. Med. Assoc. 251 (3) (1984) 351-364.

[278] The Lipid Research Clinics Program, The lipid research clinics coronary primary prevention trial. Results of 6 years of post-trial follow-up. The lipid research clinics investigators, Arch. Intern. Med. 152 (1992) 1399-1410.

[279] L. He, P. Wickremasingha, J. Lee, B. Tao, J. Mendell-Harary, J. Walker, D. Wight, Lack of effect of colesevelam $\mathrm{HCl}$ on the single-dose pharmacokinetics of aspirin, atenolol, enalapril, phenytoin, rosiglitazone, and sitagliptin, Diabetes Res. Clin. Pract. 104 (2014) 401-409.

[280] M. Abifadel, M. Varret, J.P. Rabes, D. Allard, K. Ouguerram, M. Devillers, C. Cruaud, S. Benjannet, L. Wickham, D. Erlich, A. Derre, L. Villeger, M. Farnier, I. Beucler, E. Bruckert, J. Chambaz, B. Chanu, J.M. Lecerf, G. Luc, P. Moulin, J. Weissenbach, A. Prat, M. Krempf, C. Junien, N.G. Seidah, C. Boileau, Mutations in PCSK9 cause autosomal dominant hypercholesterolemia, Nat. Genet. 34 (2003) 154-156.

[281] G.D. Norata, G. Tibolla, A.L. Catapano, Targeting PCSK9 for hypercholesterolemia, Annu. Rev. Pharmacol. Toxicol. 54 (2014) 273-293.

[282] T. Nozue, Lipid lowering therapy and circulating PCSK9 concentration, J. Atheroscler. Thromb. 24 (2017) 895-907.

[283] L. Cho, M. Rocco, D. Colquhoun, D. Sullivan, R.S. Rosenson, R. Dent, A. Xue, R. Scott, S.M. Wasserman, E. Stroes, Clinical profile of statin intolerance in the phase 3 GAUSS-2 Study, Cardiovasc. Drugs Ther. 30 (2016) 297-304.

[284] A.F. Schmidt, L.S. Pearce, J.T. Wilkins, J.P. Overington, A.D. Hingorani, J.P. Casas, PCSK9 monoclonal antibodies for the primary and secondary prevention of cardiovascular disease, Cochrane Database Syst. Rev. 4 (2017) CD011748.

[285] A. Thedrez, D.J. Blom, S. Ramin-Mangata, V. Blanchard, M. Croyal, K. Chemello, B. Nativel, M. Pichelin, B. Cariou, S. Bourane, L. Tang, M. Farnier, F.J. Raal, G. Lambert, Homozygous familial hypercholesterolemia patients with identical mutations variably express the LDLR (low-density lipoprotein receptor): implications for the efficacy of evolocumab, Arterioscler. Thromb. Vasc. Biol. 38 (2018) 592-598.

[286] J.G. Robinson, M. Farnier, M. Krempf, J. Bergeron, G. Luc, M. Averna, E.S. Stroes, G. Langslet, F.J. Raal, M. El Shahawy, M.J. Koren, N.E. Lepor, C. Lorenzato, R. Pordy, U. Chaudhari, J.J. Kastelein, ODYSSEY LONG TERM Investigators, Efficacy and safety of alirocumab in reducing lipids and cardiovascular events, N. Engl. J. Med. 372 (2015) 1489-1499.

[287] E.A. Stein, T.A. Turner, Are the PCSK9 inhibitors the panacea of atherosclerosis treatment? Expert Rev. Cardiovasc Ther. 15 (2017) 491-494.

[288] D. Gaudet, D.J. Kereiakes, J.M. McKenney, E.M. Roth, C. Hanotin, D. Gipe, Y. Du, A.C. Ferrand, H.N. Ginsberg, E.A. Stein, Effect of alirocumab, a monoclonal proprotein convertase subtilisin/kexin 9 antibody, on lipoprotein(a) concentrations (a pooled analysis of $150 \mathrm{mg}$ every two weeks dosing from phase 2 trials), Am. J. Cardiol. 114 (2014) 711-715.

[289] F.J. Raal, R.P. Giugliano, M.S. Sabatine, M.J. Koren, G. Langslet, H. Bays, D. Blom, M. Eriksson, R. Dent, S.M. Wasserman, F. Huang, A. Xue, M. Albizem, R. Scott, E.A. Stein, Reduction in lipoprotein(a) with PCSK9 monoclonal antibody evolocumab (AMG 145): a pooled analysis of more than 1,300 patients in 4 phase II trials, J. Am. Coll. Cardiol. 63 (2014) 1278-1288.

[290] M.S. Sabatine, R.P. Giugliano, S.D. Wiviott, F.J. Raal, D.J. Blom, J. Robinson, C.M. Ballantyne, R. Somaratne, J. Legg, S.M. Wasserman, R. Scott, M.J. Koren, E.A. Stein, Open-Label Study of Long-Term Evaluation against LDL Cholesterol (OSLER) Investigators. Efficacy and safety of evolocumab in reducing lipids and cardiovascular events, N. Engl. J. Med. 372 (2015) 1500-1509.

[291] E.P. Navarese, M. Kolodziejczak, D.J. Kereiakes, U.S. Tantry, C. O'Connor, P.A. Gurbel, Proprotein convertase subtilisin/kexin type 9 monoclonal antibodies for acute coronary syndrome: a narrative review, Ann. Intern. Med. 164 (2016) 600-607.

[292] A.F. Cicero, E. Tartagni, S. Ertek, Safety and tolerability of injectable lipid-lowering drugs: a review of available clinical data, Expert Opin. Drug Saf. 13 (2014) 1023-1030.

[293] M.J. Lipinski, U. Benedetto, R.O. Escarcega, G. Biondi-Zoccai, T. Lhermusier, N.C. Baker, R. Torguson, H.B. Brewer Jr., R. Waksman, The impact of proprotein convertase subtilisin-kexin type 9 serine protease inhibitors on lipid levels and outcomes in patients with primary hypercholesterolaemia: a network meta-analysis, Eur. Heart J. 37 (2016) 536-545.

[294] A.F. Schmidt, D.I. Swerdlow, M.V. Holmes, R.S. Patel, Z. Fairhurst-Hunter, D.M. Lyall, F.P. Hartwig, B.L. Horta, E. Hypponen, C. Power, M. Moldovan, E van Iperen, G.K. Hovingh, I. Demuth, K. Norman, E. Steinhagen-Thiessen, J. Demuth, L. Bertram, T. Liu, S. Coassin, J. Willeit, S. Kiechl, K. Willeit, D. Mason, J. Wright, R. Morris, G. Wanamethee, P. Whincup, Y. Ben-Shlomo, S. McLachlan, J.F. Price, M. Kivimaki, C. Welch, A. Sanchez-Galvez, P. Marques-Vidal, A. Nicolaides, A.G. Panayiotou, N.C. Onland-Moret, Y.T. van der Schouw, G. Matullo, G. Fiorito, S. Guarrera, C. Sacerdote, N.J. Wareham, C. Langenberg, R. Scott, J. Luan, M. Bobak, S. Malyutina, A. Pajak, R. Kubinova, A. Tamosiunas, H. Pikhart, L.L. Husemoen, N. Grarup, O. Pedersen, T. Hansen, A. Linneberg, K.S. Simonsen, J. Cooper, S.E. Humphries, M. Brilliant, T. Kitchner, H. Hakonarson, D.S. Carrell, C.A. McCarty, H.L. Kirchner, E.B. Larson, D.R. Crosslin, M. de Andrade, D.M. Roden, J.C. Denny, C. Carty, S. Hancock, J. Attia, E. Holliday, M. O'Donnell, S. Yusuf, M. Chong, G. Pare, P. van der Harst, M.A. Said, R.N. Eppinga, N. Verweij, H. Snieder, g LifeLines Cohort study, T. Christen, D.O. Mook-Kanamori, S. Gustafsson, L. Lind, E. Ingelsson, R. Pazoki, O. Franco, A. Hofman, A. Uitterlinden, A. Dehghan, A. Teumer, S. Baumeister, M. Dorr, M.M. Lerch, U. Volker, H. Volzke, J. Ward, J.P. Pell, D.J. Smith, T. Meade, A.H. Maitland-van der Zee, E.V. Baranova, R. Young, I. Ford, A. Campbell, S. Padmanabhan,
M.L. Bots, D.E. Grobbee, P. Froguel, D. Thuillier, B. Balkau, A. Bonnefond, B. Cariou, M. Smart, Y. Bao, M. Kumari, A. Mahajan, P.M. Ridker, D.I. Chasman, A.P. Reiner, L.A. Lange, M.D. Ritchie, F.W. Asselbergs, J.P. Casas, B.J. Keating, D. Preiss, Hingorani ADUCLEB consortiumSattar N, PCSK9 genetic variants and risk of type 2 diabetes: a Mendelian randomisation study, Lancet Diabetes Endocrinol 5 (2017) 97-105.

[295] Y.X. Cao, H.H. Liu, Q.T. Dong, S. Li, J.J. Li, Effect of proprotein convertase subtilisin/kexin type 9 (PCSK9) monoclonal antibodies on new-onset diabetes mellitus and glucose metabolism: a systematic review and meta-analysis, Diabetes Obes. Metab. 20 (2018) 1391-1398.

[296] L.S.F. de Carvalho, A.M. Campos, A.C. Sposito, Proprotein convertase subtilisin/ kexin type 9 (PCSK9) inhibitors and incident type 2 diabetes: a systematic review and meta-analysis with over 96,000 patient-years, Diabetes Care 41 (2018) 364-367.

[297] M.S. Sabatine, L.A. Leiter, S.D. Wiviott, R.P. Giugliano, P. Deedwania, G.M. De Ferrari, S.A. Murphy, J.F. Kuder, I. Gouni-Berthold, B.S. Lewis, Y. Handelsman, A.L. Pineda, N. Honarpour, A.C. Keech, P.S. Sever, T.R. Pedersen, Cardiovascular safety and efficacy of the PCSK9 inhibitor evolocumab in patients with and without diabetes and the effect of evolocumab on glycaemia and risk of new-onset diabetes: a prespecified analysis of the FOURIER randomised controlled trial, Lancet Diabetes Endocrinol 5 (2017) 941-950.

[298] X.L. Zhang, L. Zhu, Z.H. Wei, Q.Q. Zhu, J.Z. Qiao, Q. Dai, W. Huang, X.H. Li, J. Xie, L.N. Kang, L. Wang, B. Xu, Comparative efficacy and safety of everolimus-eluting bioresorbable scaffold versus everolimus-eluting metallic stents: a systematic review and meta-analysis, Ann. Intern. Med. 164 (2016) 752-763.

[299] R.P. Giugliano, C.P. Cannon, M.A. Blazing, J.C. Nicolau, R. Corbalan, J. Spinar, J.G. Park, J.A. White, E.A. Bohula, E. Braunwald, IMPROVE-IT (improved reduc tion of outcomes: Vytorin efficacy international trial) investigators. Benefit of adding ezetimibe to statin therapy on cardiovascular outcomes and safety in patients with versus without diabetes mellitus: results from IMPROVE-IT (improved reduction of outcomes: Vytorin efficacy international trial), Circulation 137 (2018) 1571-1582.

[300] P.M. Ridker, J.C. Tardif, P. Amarenco, W. Duggan, R.J. Glynn, J.W. Jukema, J.J.P. Kastelein, A.M. Kim, W. Koenig, S. Nissen, J. Revkin, L.M. Rose, R.D. Santos, P.F. Schwartz, C.L. Shear, C. Yunis, S.P.I.R.E. Investigators, Lipid-reduction variability and antidrug-antibody formation with bococizumab, N. Engl. J. Med. 376 (2017) 1517-1526.

[301] M. Cuchel, E.A. Meagher, H. du Toit Theron, D.J. Blom, A.D. Marais, R.A. Hegele, M.R. Averna, C.R. Sirtori, P.K. Shah, D. Gaudet, C. Stefanutti, G.B. Vigna, A.M. Du Plessis, K.J. Propert, W.J. Sasiela, L.T. Bloedon, D.J. Rader, Phase 3 HoFH Lomitapide Study investigators. Efficacy and safety of a microsomal triglyceride transfer protein inhibitor in patients with homozygous familial hypercholesterolaemia: a single-arm, open-label, phase 3 study, Lancet 381 (2013) 40-46.

[302] M. Cuchel, L.T. Bloedon, P.O. Szapary, D.M. Kolansky, M.L. Wolfe, A. Sarkis, J.S. Millar, K. Ikewaki, E.S. Siegelman, R.E. Gregg, D.J. Rader, Inhibition of microsomal triglyceride transfer protein in familial hypercholesterolemia, N. Engl. J. Med. 356 (2007) 148-156.

[303] A. Agarwala, P. Jones, V. Nambi, The role of antisense oligonucleotide therapy in patients with familial hypercholesterolemia: risks, benefits, and management recommendations, Curr. Atheroscler. Rep. 17 (2015) 467.

[304] N. Li, Q. Li, X.Q. Tian, H.Y. Qian, Y.J. Yang, Mipomersen is a promising therapy in the management of hypercholesterolemia: a meta-analysis of randomized controlled trials, Am. J. Cardiovasc. Drugs 14 (2014) 367-376.

[305] M.J. Chapman, J.S. Redfern, M.E. McGovern, P. Giral, Niacin and fibrates in atherogenic dyslipidemia: pharmacotherapy to reduce cardiovascular risk, Pharmacol. Ther. 126 (2010) 314-345.

[306] Accord Study Group, H.N. Ginsberg, M.B. Elam, L.C. Lovato, J.R. Crouse III, L.A. Leiter, P. Linz, W.T. Friedewald, J.B. Buse, H.C. Gerstein, J. Probstfield, R.H. Grimm, F. Ismail-Beigi, J.T. Bigger, D.C. Goff Jr., W.C. Cushman, D.G. Simons-Morton, R.P. Byington, Effects of combination lipid therapy in type 2 diabetes mellitus, N. Engl. J. Med. 362 (2010) 1563-1574.

[307] A. Keech, R.J. Simes, P. Barter, J. Best, R. Scott, M.R. Taskinen, P. Forder, A. Pillai, T. Davis, P. Glasziou, P. Drury, Y.A. Kesaniemi, D. Sullivan, D. Hunt, P. Colman, M. d'Emden, M. Whiting, C. Ehnholm, M. Laakso, FIELD study investigators. Effects of long-term fenofibrate therapy on cardiovascular events in 9795 people with type 2 diabetes mellitus (the FIELD study): randomised controlled trial, Lancet 366 (2005) 1849-1861.

[308] M.H. Frick, O. Elo, K. Haapa, O.P. Heinonen, P. Heinsalmi, P. Helo, J.K. Huttunen, P. Kaitaniemi, P. Koskinen, V. Manninen, Helsinki Heart Study: primary-prevention trial with gemfibrozil in middle-aged men with dyslipidemia. Safety of treatment, changes in risk factors, and incidence of coronary heart disease, N. Engl. J. Med. 317 (1987) 1237-1245.

[309] H.B. Rubins, S.J. Robins, D. Collins, C.L. Fye, J.W. Anderson, M.B. Elam, F.H. Faas, E. Linares, E.J. Schaefer, G. Schectman, T.J. Wilt, J. Wittes, Gemfibrozil for the secondary prevention of coronary heart disease in men with low levels of highdensity lipoprotein cholesterol. Veterans Affairs High-Density Lipoprotein Cholesterol Intervention Trial Study Group, N. Engl. J. Med. 341 (1999) 410-418.

[310] Lipids and lipoproteins in symptomatic coronary heart disease. Distribution, intercorrelations, and significance for risk classification in 6,700 men and 1,500 women. The Bezafibrate Infarction Prevention (BIP) Study Group, Israel, Circulation 86 (1992) 839-848.

[311] T. Meade, R. Zuhrie, C. Cook, J. Cooper, Bezafibrate in men with lower extremity arterial disease: randomised controlled trial, BMJ 325 (2002) 1139.

[312] C.K. Ip, D.M. Jin, J.J. Gao, Z. Meng, J. Meng, Z. Tan, J.F. Wang, D.F. Geng, Effects of add-on lipid-modifying therapy on top of background statin treatment on major cardiovascular events: a meta-analysis of randomized controlled trials, Int. J. 
Cardiol. 191 (2015) 138-148.

[313] D. Keene, C. Price, M.J. Shun-Shin, D.P. Francis, Effect on cardiovascular risk of high density lipoprotein targeted drug treatments niacin, fibrates, and CETP inhibitors: meta-analysis of randomised controlled trials including 117,411 patients, BMJ 349 (2014) g4379.

[314] E. Bruckert, J. Labreuche, D. Deplanque, P.J. Touboul, P. Amarenco, Fibrates effect on cardiovascular risk is greater in patients with high triglyceride levels or atherogenic dyslipidemia profile: a systematic review and meta-analysis, J. Cardiovasc. Pharmacol. 57 (2011) 267-272.

[315] M. Jun, B. Zhu, M. Tonelli, M.J. Jardine, A. Patel, B. Neal, T. Liyanage, A. Keech, A. Cass, V. Perkovic, Effects of fibrates in kidney disease: a systematic review and meta-analysis, J. Am. Coll. Cardiol. 60 (2012) 2061-2071.

[316] M. Lee, J.L. Saver, A. Towfighi, J. Chow, B. Ovbiagele, Efficacy of fibrates for cardiovascular risk reduction in persons with atherogenic dyslipidemia: a metaanalysis, Atherosclerosis 217 (2011) 492-498.

[317] J.C. Fruchart, Pemafibrate (K-877), a novel selective peroxisome proliferator-ac tivated receptor alpha modulator for management of atherogenic dyslipidaemia Cardiovasc. Diabetol. 16 (2017) 124.

[318] A.D. Pradhan, N.P. Paynter, B.M. Everett, R.J. Glynn, P. Amarenco, M. Elam, H. Ginsberg, W.R. Hiatt, S. Ishibashi, W. Koenig, B.G. Nordestgaard, J.C. Fruchart, P. Libby, P.M. Ridker, Rationale and design of the pemafibrate to reduce cardiovascular outcomes by reducing triglycerides in patients with diabetes (PROMINENT) study, Am. Heart J. 206 (2018) 80-93.

[319] M.H. Davidson, A. Armani, J.M. McKenney, T.A. Jacobson, Safety considerations with fibrate therapy, Am. J. Cardiol. 99 (2007) 3C-18C.

[320] D.J. Graham, J.A. Staffa, D. Shatin, S.E. Andrade, S.D. Schech, L. La Grenade, J.H. Gurwitz, K.A. Chan, M.J. Goodman, R. Platt, Incidence of hospitalized rhabdomyolysis in patients treated with lipid-lowering drugs, J. Am. Med. Assoc. 292 (2004) 2585-2590.

[321] D. Preiss, M.J. Tikkanen, P. Welsh, I. Ford, L.C. Lovato, M.B. Elam, J.C. LaRosa, D.A. DeMicco, H.M. Colhoun, I. Goldenberg, M.J. Murphy, T.M. MacDonald, T.R. Pedersen, A.C. Keech, P.M. Ridker, J. Kjekshus, N. Sattar, J.J. McMurray, Lipid-modifying therapies and risk of pancreatitis: a meta-analysis, J. Am. Med. Assoc. 308 (2012) 804-811.

[322] M.R. Taskinen, D.R. Sullivan, C. Ehnholm, M. Whiting, D. Zannino, R.J. Simes, A.C. Keech, P.J. Barter, FIELD study investigators. Relationships of HDL cholesterol, ApoA-I, and ApoA-II with homocysteine and creatinine in patients with type 2 diabetes treated with fenofibrate, Arterioscler. Thromb. Vasc. Biol. 29 (2009) 950-955.

[323] E.M. Balk, A.H. Lichtenstein, M. Chung, B. Kupelnick, P. Chew, J. Lau, Effects of omega-3 fatty acids on serum markers of cardiovascular disease risk: a systematic review, Atherosclerosis 189 (2006) 19-30.

[324] C.M. Ballantyne, H.E. Bays, J.J. Kastelein, E. Stein, J.L. Isaacsohn, R.A. Braeckman, P.N. Soni, Efficacy and safety of eicosapentaenoic acid ethyl ester (AMR101) therapy in statin-treated patients with persistent high triglycerides (from the ANCHOR study), Am. J. Cardiol. 110 (2012) 984-992.

[325] H.E. Bays, C.M. Ballantyne, J.J. Kastelein, J.L. Isaacsohn, R.A. Braeckman, P.N. Soni, Eicosapentaenoic acid ethyl ester (AMR101) therapy in patients with very high triglyceride levels (from the Multi-center, plAcebo-controlled, Randomized, double-blind, 12-week study with an open-label Extension [MARINE] trial), Am. J. Cardiol. 108 (2011) 682-690.

[326] J.J. Kastelein, K.C. Maki, A. Susekov, M. Ezhov, B.G. Nordestgaard, B.N. Machielse, D. Kling, M.H. Davidson, Omega-3 free fatty acids for the treatment of severe hypertriglyceridemia: the EpanoVa fOr Lowering Very high triglyceridEs (EVOLVE) trial, J Clin Lipidol 8 (2014) 94-106.

[327] E.S.G. Stroes, A.V. Susekov, T.W.A. de Bruin, M. Kvarnstrom, H. Yang, M.H. Davidson, Omega-3 carboxylic acids in patients with severe hypertriglycer idemia: EVOLVE II, a randomized, placebo-controlled trial, J Clin Lipidol 12 (2018) 321-330.

[328] A.S. Abdelhamid, T.J. Brown, J.S. Brainard, P. Biswas, G.C. Thorpe, H.J. Moore, K.H. Deane, F.K. AlAbdulghafoor, C.D. Summerbell, H.V. Worthington, F. Song, L. Hooper, Omega-3 fatty acids for the primary and secondary prevention of cardiovascular disease, Cochrane Database Syst. Rev. 7 (2018) CD003177.

[329] Ascend Study Collaborative Group, L. Bowman, M. Mafham, K. Wallendszus, W. Stevens, G. Buck, J. Barton, K. Murphy, T. Aung, R. Haynes, J. Cox, A. Murawska, A. Young, M. Lay, F. Chen, E. Sammons, E. Waters, A. Adler, J. Bodansky, A. Farmer, R. McPherson, A. Neil, D. Simpson, R. Peto, C. Baigent, R. Collins, S. Parish, J. Armitage, Effects of n-3 fatty acid supplements in diabetes mellitus, N. Engl. J. Med. 379 (2018) 1540-1550.

[330] L. Hooper, R.L. Thompson, R.A. Harrison, C.D. Summerbell, A.R. Ness, H.J. Moore, H.V. Worthington, P.N. Durrington, J.P. Higgins, N.E. Capps, R.A. Riemersma, S.B. Ebrahim, G. Davey Smith, Risks and benefits of omega 3 fats for mortality, cardiovascular disease, and cancer: systematic review, BMJ 332 (2006) 752-760.

[331] R. Marchioli, F. Barzi, E. Bomba, C. Chieffo, D. Di Gregorio, R. Di Mascio, M.G. Franzosi, E. Geraci, G. Levantesi, A.P. Maggioni, L. Mantini, R.M. Marfisi, G. Mastrogiuseppe, N. Mininni, G.L. Nicolosi, M. Santini, C. Schweiger, L. Tavazzi, G. Tognoni, C. Tucci, F. Valagussa, GISSI-Prevenzione Investigators, Early protection against sudden death by n-3 polyunsaturated fatty acids after myocardial infarction: time-course analysis of the results of the Gruppo Italiano per lo Studio della Sopravvivenza nell'Infarto Miocardico (GISSI)-Prevenzione, Circulation 105 (2002) 1897-1903.

[332] S.J. Nicholls, A.M. Lincoff, D. Bash, C.M. Ballantyne, P.J. Barter, M.H. Davidson, J.J.P. Kastelein, W. Koenig, D.K. McGuire, D. Mozaffarian, T.R. Pedersen, P.M. Ridker, K. Ray, B.W. Karlson, T. Lundstrom, K. Wolski, S.E. Nissen, Assessment of omega-3 carboxylic acids in statin-treated patients with high levels of triglycerides and low levels of high-density lipoprotein cholesterol: rationale and design of the STRENGTH trial, Clin. Cardiol. 41 (2018) 1281-1288.

[333] J.E. Manson, N.R. Cook, I.M. Lee, W. Christen, S.S. Bassuk, S. Mora, H. Gibson, C.M. Albert, D. Gordon, T. Copeland, D. D'Agostino, G. Friedenberg, C. Ridge, V. Bubes, E.L. Giovannucci, W.C. Willett, J.E. Buring, VITAL Research Group, Marine n-3 fatty acids and prevention of cardiovascular disease and cancer, $\mathrm{N}$. Engl. J. Med. 380 (2019) 23-32.

[334] T.M. Brasky, A.K. Darke, X. Song, C.M. Tangen, P.J. Goodman, I.M. Thompson, F.L. Meyskens Jr., G.E. Goodman, L.M. Minasian, H.L. Parnes, E.A. Klein, A.R. Kristal, Plasma phospholipid fatty acids and prostate cancer risk in the SELECT trial, J. Natl. Cancer Inst. 105 (2013) 1132-1141.

[335] V.S. Kamanna, M.L. Kashyap, Mechanism of action of niacin, Am. J. Cardiol. 101 (2008) 20B-26B.

[336] P.J. Barter, M. Caulfield, M. Eriksson, S.M. Grundy, J.J. Kastelein, M. Komajda, J. Lopez-Sendon, L. Mosca, J.C. Tardif, D.D. Waters, C.L. Shear, J.H. Revkin, K.A. Buhr, M.R. Fisher, A.R. Tall, B. BrewerILLUMINATE Investigators, Effects of torcetrapib in patients at high risk for coronary events, N. Engl. J. Med. 357 (2007) 2109-2122.

[337] B.A. Ference, J.J.P. Kastelein, H.N. Ginsberg, M.J. Chapman, S.J. Nicholls, K.K. Ray, C.J. Packard, U. Laufs, R.D. Brook, C. Oliver-Williams, A.S. Butterworth, J. Danesh, G.D. Smith, A.L. Catapano, M.S. Sabatine, Association of genetic variants related to CETP inhibitors and statins with lipoprotein levels and cardiovascular risk, J. Am. Med. Assoc. 318 (2017) 947-956.

[338] K. Fitzgerald, S. White, A. Borodovsky, B.R. Bettencourt, A. Strahs, V. Clausen, P. Wijngaard, J.D. Horton, J. Taubel, A. Brooks, C. Fernando, R.S. Kauffman, D. Kallend, A. Vaishnaw, A. Simon, A highly durable RNAi therapeutic inhibitor of PCSK9, N. Engl. J. Med. 376 (2017) 41-51.

[339] K.K. Ray, U. Landmesser, L.A. Leiter, D. Kallend, R. Dufour, M. Karakas, T. Hall, R.P. Troquay, T. Turner, F.L. Visseren, P. Wijngaard, R.S. Wright, J.J. Kastelein, Inclisiran in patients at high cardiovascular risk with elevated LDL cholesterol, $\mathrm{N}$. Engl. J. Med. 376 (2017) 1430-1440.

[340] A. Saeed, C.M. Ballantyne, Bempedoic acid (ETC-1002): a current review, Cardiol. Clin. 36 (2018) 257-264

[341] C.M. Ballantyne, M. Banach, G.B.J. Mancini, N.E. Lepor, J.C. Hanselman, X. Zhao, L.A. Leiter, Efficacy and safety of bempedoic acid added to ezetimibe in statinintolerant patients with hypercholesterolemia: a randomized, placebo-controlled study, Atherosclerosis 277 (2018) 195-203.

[342] K.K. Ray, H.E. Bays, A.L. Catapano, N.D. Lalwani, L.T. Bloedon, L.R. Sterling, P.L. Robinson, C.M. Ballantyne, CLEAR Harmony Trial, Safety and efficacy of bempedoic acid to reduce LDL cholesterol, N. Engl. J. Med. 380 (2019) 1022-1032.

[343] N.O. Stitziel, A.V. Khera, X. Wang, A.J. Bierhals, A.C. Vourakis, A.E. Sperry, P. Natarajan, D. Klarin, C.A. Emdin, S.M. Zekavat, A. Nomura, J. Erdmann, H. Schunkert, N.J. Samani, W.E. Kraus, S.H. Shah, B. Yu, E. Boerwinkle, D.J. Rader, N. Gupta, P.M. Frossard, A. Rasheed, J. Danesh, E.S. Lander, S. Gabriel, D. Saleheen, K. Musunuru, S. Kathiresan, PROMIS and Myocardial Infarction Genetics Consortium Investigators. ANGPTL3 deficiency and protection against coronary artery disease, J. Am. Coll. Cardiol. 69 (2017) 2054-2063.

[344] D. Gaudet, D.A. Gipe, R. Pordy, Z. Ahmad, M. Cuchel, P.K. Shah, K.Y. Chyu, W.J. Sasiela, K.C. Chan, D. Brisson, E. Khoury, P. Banerjee, V. Gusarova, J. Gromada, N. Stahl, G.D. Yancopoulos, G.K. Hovingh, ANGPTL3 inhibition in homozygous familial hypercholesterolemia, N. Engl. J. Med. 377 (2017) 296-297.

[345] M.J. Graham, R.G. Lee, T.A. Brandt, L.J. Tai, W. Fu, R. Peralta, R. Yu, E. Hurh, E. Paz, B.W. McEvoy, B.F. Baker, N.C. Pham, A. Digenio, S.G. Hughes, R.S. Geary, J.L. Witztum, R.M. Crooke, S. Tsimikas, Cardiovascular and metabolic effects of ANGPTL3 antisense oligonucleotides, N. Engl. J. Med. 377 (2017) 222-232.

[346] M.J. Graham, R.G. Lee, T.A. Bell III, W. Fu, A.E. Mullick, V.J. Alexander, W. Singleton, N. Viney, R. Geary, J. Su, B.F. Baker, J. Burkey, S.T. Crooke, R.M. Crooke, Antisense oligonucleotide inhibition of apolipoprotein C-III reduces plasma triglycerides in rodents, nonhuman primates, and humans, Circ. Res. 112 (2013) 1479-1490.

[347] I. Gouni-Berthold, V. Alexander, A. Digenio, R. DuFour, E. Steinhagen-Thiessen, S. Martin, P. Moriarty, S. Hughes, R. Jones, J.L. Witztum, D. Gaudet, Apolipoprotein C-III inhibition with volanesorsen in patients with hypertriglyceridemia (COMPASS): a randomized, double-blind, placebo-controlled trial, Atherosclerosis Suppl. 28 (2017) e1-e2.

[348] D. Gaudet, A. Digenio, V. Alexander, M. Arca, A. Jones, E. Stroes, J. Bergeron, F. Civeira, L. Hemphill, D. Blom, J. Flaim, S. Hughes, R. Geary, S. Tsimikas, J. Witztum, E. Bruckert, The approach study: a randomized, double-blind, placebo-controlled, phase 3 study of volanesorsen administered subcutaneously to patients with familial chylomicronemia syndrome (FCS), Atherosclerosis 263 (2017) e10.

[349] N.A. Rocha, C. East, J. Zhang, P.A. McCullough, ApoCIII as a cardiovascular risk factor and modulation by the novel lipid-lowering agent volanesorsen, Curr. Atheroscler. Rep. 19 (2017) 62.

[350] S.A. Didichenko, A.V. Navdaev, A.M. Cukier, A. Gille, P. Schuetz, M.O. Spycher, P. Therond, M.J. Chapman, A. Kontush, S.D. Wright, Enhanced HDL functionality in small HDL species produced upon remodeling of HDL by reconstituted HDL, CSL112: effects on cholesterol efflux, anti-inflammatory and antioxidative activity, Circ. Res. 119 (2016) 751-763.

[351] A. Digenio, R.L. Dunbar, V.J. Alexander, M. Hompesch, L. Morrow, R.G. Lee, M.J. Graham, S.G. Hughes, R. Yu, W. Singleton, B.F. Baker, S. Bhanot, R.M. Crooke, Antisense-mediated lowering of plasma apolipoprotein C-III by volanesorsen improves dyslipidemia and insulin sensitivity in type 2 diabetes, Diabetes Care 39 (2016) 1408-1415.

[352] N.J. Viney, J.C. van Capelleveen, R.S. Geary, S. Xia, J.A. Tami, R.Z. Yu, S.M. Marcovina, S.G. Hughes, M.J. Graham, R.M. Crooke, S.T. Crooke, 
J.L. Witztum, E.S. Stroes, S. Tsimikas, Antisense oligonucleotides targeting apolipoprotein(a) in people with raised lipoprotein(a): two randomised, double-blind, placebo-controlled, dose-ranging trials, Lancet 388 (2016) 2239-2253.

[354] K.C. Maki, J.R. Guyton, C.E. Orringer, I. Hamilton-Craig, D.D. Alexander, M.H. Davidson, Triglyceride-lowering therapies reduce cardiovascular disease event risk in subjects with hypertriglyceridemia, J Clin Lipidol 10 (2016) 905-914.

[357] Global Lipids Genetics Consortium, C.J. Willer, E.M. Schmidt, S. Sengupta, G.M. Peloso, S. Gustafsson, S. Kanoni, A. Ganna, J. Chen, M.L. Buchkovich, S. Mora, J.S. Beckmann, J.L. Bragg-Gresham, H.Y. Chang, A. Demirkan, H.M. Den Hertog, R. Do, L.A. Donnelly, G.B. Ehret, T. Esko, M.F. Feitosa, T. Ferreira, K. Fischer, P. Fontanillas, R.M. Fraser, D.F. Freitag, D. Gurdasani, K. Heikkila, E. Hypponen, A. Isaacs, A.U. Jackson, A. Johansson, T. Johnson, M. Kaakinen, J. Kettunen, M.E. Kleber, X. Li, J. Luan, L.P. Lyytikainen, P.K. Magnusson, M. Mangino, E. Mihailov, M.E. Montasser, M. Muller-Nurasyid, I.M. Nolte, J.R. O'Connell, C.D. Palmer, M. Perola, A.K. Petersen, S. Sanna, R. Saxena, S.K. Service, S. Shah, D. Shungin, C. Sidore, C. Song, R.J. Strawbridge, I. Surakka, T. Tanaka, T.M. Teslovich, G. Thorleifsson, E.G. Van den Herik, B.F. Voight, K.A. Volcik, L.L. Waite, A. Wong, Y. Wu, W. Zhang, D. Absher, G. Asiki, I. Barroso, L.F. Been, J.L. Bolton, L.L. Bonnycastle, P. Brambilla, M.S. Burnett, G. Cesana, M. Dimitriou, A.S. Doney, A. Doring, P. Elliott, S.E. Epstein, G.I. Eyjolfsson, B. Gigante, M.O. Goodarzi, H. Grallert, M.L. Gravito, C.J. Groves, G. Hallmans, A.L. Hartikainen, C. Hayward, D. Hernandez, A.A. Hicks, H. Holm, Y.J. Hung, T. Illig, M.R. Jones, P. Kaleebu, J.J. Kastelein, K.T. Khaw, E. Kim, N. Klopp, P. Komulainen, M. Kumari, C. Langenberg, T. Lehtimaki, S.Y. Lin, J. Lindstrom, R.J. Loos, F. Mach, W.L. McArdle, C. Meisinger, B.D. Mitchell, G. Muller, R. Nagaraja, N. Narisu, T.V. Nieminen, R.N. Nsubuga, I. Olafsson, K.K. Ong, A. Palotie, T. Papamarkou, C. Pomilla, A. Pouta, D.J. Rader, M.P. Reilly, P.M. Ridker, F. Rivadeneira, I. Rudan, A. Ruokonen, N. Samani, H. Scharnagl, J. Seeley, K. Silander, A. Stancakova, K. Stirrups, A.J. Swift, L. Tiret, A.G. Uitterlinden, L.J. van Pelt, S. Vedantam, N. Wainwright, C. Wijmenga, S.H. Wild, G. Willemsen, T. Wilsgaard, J.F. Wilson, E.H. Young, J.H. Zhao, L.S. Adair, D. Arveiler, T.L. Assimes, S. Bandinelli, F. Bennett, M. Bochud, B.O. Boehm, D.I. Boomsma, I.B. Borecki, S.R. Bornstein, P. Bovet, M. Burnier, H. Campbell, A. Chakravarti, J.C. Chambers, Y.D. Chen, F.S. Collins, R.S. Cooper, J. Danesh, G. Dedoussis, U. de Faire, A.B. Feranil, J. Ferrieres, L. Ferrucci, N.B. Freimer, C. Gieger, L.C. Groop, V. Gudnason, U. Gyllensten, A. Hamsten, T.B. Harris, A. Hingorani, J.N. Hirschhorn, A. Hofman, G.K. Hovingh, C.A. Hsiung, S.E. Humphries, S.C. Hunt, K. Hveem, C. Iribarren, M.R. Jarvelin, A. Jula, M. Kahonen, J. Kaprio, A. Kesaniemi, M. Kivimaki, J.S. Kooner, P.J. Koudstaal, R.M. Krauss, D. Kuh, J. Kuusisto, K.O. Kyvik, M. Laakso, T.A. Lakka, L. Lind, C.M. Lindgren, N.G. Martin, W. Marz, M.I. McCarthy, C.A. McKenzie, P. Meneton, A. Metspalu, L. Moilanen, A.D. Morris, P.B. Munroe, I. Njolstad, N.L. Pedersen, C. Power, P.P. Pramstaller, J.F. Price, B.M. Psaty, T. Quertermous, R. Rauramaa, D. Saleheen, V. Salomaa, D.K. Sanghera, J. Saramies, P.E. Schwarz, W.H. Sheu, A.R. Shuldiner, A. Siegbahn, T.D. Spector, K. Stefansson, D.P. Strachan, B.O. Tayo, E. Tremoli, J. Tuomilehto, M. Uusitupa, C.M. van Duijn, P. Vollenweider, L. Wallentin, N.J. Wareham, J.B. Whitfield, B.H. Wolffenbuttel, J.M. Ordovas, E. Boerwinkle, C.N. Palmer, U. Thorsteinsdottir, D.I. Chasman, J.I. Rotter, P.W. Franks, S. Ripatti, L.A. Cupples, M.S. Sandhu, S.S. Rich, M. Boehnke, P. Deloukas, S. Kathiresan, K.L. Mohlke, E. Ingelsson, G.R. Abecasis, Discovery and refinement of loci associated with lipid levels, Nat. Genet. 45 (2013) 1274-1283.

[358] A.J. Brahm, R.A. Hegele, Combined hyperlipidemia: familial but not (usually) monogenic, Curr. Opin. Lipidol. 27 (2016) 131-140.

[359] P. Ripatti, J.T. Ramo, S. Soderlund, I. Surakka, N. Matikainen, M. Pirinen, P. Pajukanta, A.P. Sarin, S.K. Service, P.P. Laurila, C. Ehnholm, V. Salomaa, R.K. Wilson, A. Palotie, N.B. Freimer, M.R. Taskinen, S. Ripatti, The contribution of GWAS loci in familial dyslipidemias, PLoS Genet. 12 (2016) e1006078.

[360] M.J. Veerkamp, J. de Graaf, S.J. Bredie, J.C. Hendriks, P.N. Demacker, A.F. Stalenhoef, Diagnosis of familial combined hyperlipidemia based on lipid phenotype expression in 32 families: results of a 5-year follow-up study, Arterioscler. Thromb. Vasc. Biol. 22 (2002) 274-282.

[362] S.D. de Ferranti, A.M. Rodday, M.M. Mendelson, J.B. Wong, L.K. Leslie, R.C. Sheldrick, Prevalence of familial hypercholesterolemia in the 1999 to 2012 United States national health and nutrition examination surveys (NHANES), Circulation 133 (2016) 1067-1072.

[363] B.G. Nordestgaard, M.J. Chapman, S.E. Humphries, H.N. Ginsberg, L. Masana, O.S. Descamps, O. Wiklund, R.A. Hegele, F.J. Raal, J.C. Defesche, A. Wiegman, R.D. Santos, G.F. Watts, K.G. Parhofer, G.K. Hovingh, P.T. Kovanen, C. Boileau, M. Averna, J. Borén, E. Bruckert, A.L. Catapano, J.A. Kuivenhoven, P. Pajukanta, K. Ray, A.F. Stalenhoef, E. Stroes, M.R. Taskinen, A. Tybjærg-Hansen, European Atherosclerosis Society Consensus Panel. Familial hypercholesterolaemia is underdiagnosed and undertreated in the general population: guidance for clinicians to prevent coronary heart disease: consensus statement of the European Atherosclerosis Society, Eur. Heart J. 34 (2013) 3478-3490a.

[364] Risk of fatal coronary heart disease in familial hypercholesterolaemia. Scientific Steering Committee on behalf of the Simon Broome Register Group, BMJ 303 (1991) 893-896.

[365] World Health Organization, Human genetics programme. Familial hypercholesterolemia: report of a second WHO consultation. WHO/HGN/FH/Cons/99.2. Geneva, World Health Organization (1999), https://apps.who.int/iris/handle/ 10665/66346, Accessed date: 17 July 2019.

[366] U. Landmesser, M.J. Chapman, M. Farnier, B. Gencer, S. Gielen, G.K. Hovingh, T.F. Luscher, D. Sinning, L. Tokgozoglu, O. Wiklund, J.L. Zamorano, F.J. Pinto, A.L. Catapano, European Society of Cardiology (ESC); European Atherosclerosis Society (EAS). European Society of Cardiology/European Atherosclerosis Society
Task Force consensus statement on proprotein convertase subtilisin/kexin type 9 inhibitors: practical guidance for use in patients at very high cardiovascular risk, Eur. Heart J. 38 (2017) 2245-2255.

[367] P.M. Ridker, J. Revkin, P. Amarenco, R. Brunell, M. Curto, F. Civeira, M. Flather, R.J. Glynn, J. Gregoire, J.W. Jukema, Y. Karpov, J.J.P. Kastelein, W. Koenig, A. Lorenzatti, P. Manga, U. Masiukiewicz, M. Miller, A. Mosterd, J. Murin, J.C. Nicolau, S. Nissen, P. Ponikowski, R.D. Santos, P.F. Schwartz, H. Soran, H. White, R.S. Wright, M. Vrablik, C. Yunis, C.L. Shear, J.C. Tardif, SPIRE Cardiovascular Outcome Investigators. Cardiovascular efficacy and safety of bococizumab in high-risk patients, N. Engl. J. Med. 376 (2017) 1527-1539.

[368] M. Cuchel, E. Bruckert, H.N. Ginsberg, F.J. Raal, R.D. Santos, R.A. Hegele, J.A. Kuivenhoven, B.G. Nordestgaard, O.S. Descamps, E. Steinhagen-Thiessen, A. Tybjaerg-Hansen, G.F. Watts, M. Averna, C. Boileau, J. Boren, A.L. Catapano, J.C. Defesche, G.K. Hovingh, S.E. Humphries, P.T. Kovanen, L. Masana, P. Pajukanta, K.G. Parhofer, K.K. Ray, A.F. Stalenhoef, E. Stroes, M.R. Taskinen, A. Wiegman, O. Wiklund, M.J. Chapman, European atherosclerosis society consensus panel on familial hypercholesterolaemia. Homozygous familial hypercholesterolaemia: new insights and guidance for clinicians to improve detection and clinical management. A position paper from the consensus panel on familial hypercholesterolaemia of the European atherosclerosis society, Eur. Heart J. 35 (2014) 2146-2157.

[369] A. Wiegman, S.S. Gidding, G.F. Watts, M.J. Chapman, H.N. Ginsberg, M. Cuchel, L. Ose, M. Averna, C. Boileau, J. Boren, E. Bruckert, A.L. Catapano, J.C. Defesche, O.S. Descamps, R.A. Hegele, G.K. Hovingh, S.E. Humphries, P.T. Kovanen, J.A. Kuivenhoven, L. Masana, B.G. Nordestgaard, P. Pajukanta, K.G. Parhofer, F.J. Raal, K.K. Ray, R.D. Santos, A.F. Stalenhoef, E. Steinhagen-Thiessen, E.S. Stroes, M.R. Taskinen, A. Tybjaerg-Hansen, O. Wiklund, European Atherosclerosis Society Consensus Panel. Familial hypercholesterolaemia in children and adolescents: gaining decades of life by optimizing detection and treatment, Eur. Heart J. 36 (2015) 2425-2437.

[370] M. Braamskamp, G. Langslet, B.W. McCrindle, D. Cassiman, G.A. Francis, C. Gagne, D. Gaudet, K.M. Morrison, A. Wiegman, T. Turner, E. Miller, D.M. Kusters, J.S. Raichlen, P.D. Martin, E.A. Stein, J.J.P. Kastelein, B.A. Hutten, Effect of rosuvastatin on carotid intima-media thickness in children with heterozygous familial hypercholesterolemia: the CHARON study (Hypercholesterolemia in Children and Adolescents Taking Rosuvastatin Open Label), Circulation 136 (2017) 359-366.

[371] A. Vuorio, J. Kuoppala, P.T. Kovanen, S.E. Humphries, S. Tonstad, A. Wiegman, E. Drogari, U. Ramaswami, Statins for children with familial hypercholesterolemia, Cochrane Database Syst. Rev. 7 (2017) CD006401.

[372] Z. Reiner, Treatment of children with homozygous familial hypercholester olaemia, Eur J Prev Cardiol 25 (2018) 1095-1097.

[373] C. Koopal, A.D. Marais, F.L. Visseren, Familial dysbetalipoproteinemia: an underdiagnosed lipid disorder, Curr. Opin. Endocrinol. Diabetes Obes. 24 (2017) 133-139.

[374] R.W. Mahley, K.H. Weisgraber, Y. Huang, Apolipoprotein E: structure determines function, from atherosclerosis to Alzheimer's disease to AIDS, J. Lipid Res. 50 (2009) S183-S188.

[375] D. Marais, Dysbetalipoproteinemia: an extreme disorder of remnant metabolism, Curr. Opin. Lipidol. 26 (2015) 292-297.

[376] A. Sniderman, P. Couture, J. de Graaf, Diagnosis and treatment of apolipoprotein B dyslipoproteinemias, Nat. Rev. Endocrinol. 6 (2010) 335-346.

[377] A.J. Brahm, R.A. Hegele, Chylomicronaemia-current diagnosis and future therapies, Nat. Rev. Endocrinol. 11 (2015) 352-362.

[378] R.C. Bauer, S.A. Khetarpal, N.J. Hand, D.J. Rader, Therapeutic targets of triglyceride metabolism as informed by human genetics, Trends Mol. Med. 22 (2016) 328-340.

[379] D. Gaudet, J. Methot, S. Dery, D. Brisson, C. Essiembre, G. Tremblay, K. Tremblay, J. de Wal, J. Twisk, N. van den Bulk, V. Sier-Ferreira, S. van Deventer, Efficacy and long-term safety of alipogene tiparvovec (AAV1-LPLS447X) gene therapy for lipoprotein lipase deficiency: an open-label trial, Gene Ther. 20 (2013) 361-369.

[380] M.W. Huff, R.A. Hegele, Apolipoprotein C-III: going back to the future for a lipid drug target, Circ. Res. 112 (2013) 1405-1408.

[381] P. Moulin, R. Dufour, M. Averna, M. Arca, A.B. Cefalu, D. Noto, L. D'Erasmo, A. Di Costanzo, C. Marcais, L.A. Alvarez-Sala Walther, M. Banach, J. Boren, R. Cramb, I. Gouni-Berthold, E. Hughes, C. Johnson, X. Pinto, Z. Reiner, J.R. van Lennep, H. Soran, C. Stefanutti, E. Stroes, E. Bruckert, Identification and diagnosis of patients with familial chylomicronaemia syndrome (FCS): expert panel recommendations and proposal of an "FCS score, Atherosclerosis 275 (2018) 265-272.

[382] C.D. Meyers, K. Tremblay, A. Amer, J. Chen, L. Jiang, D. Gaudet, Effect of the DGAT1 inhibitor pradigastat on triglyceride and apoB48 levels in patients with familial chylomicronemia syndrome, Lipids Health Dis. 14 (2015) 8.

[383] B. Lindkvist, S. Appelros, S. Regner, J. Manjer, A prospective cohort study on risk of acute pancreatitis related to serum triglycerides, cholesterol and fasting glucose, Pancreatology 12 (2012) 317-324.

[384] N. Ewald, P.D. Hardt, H.U. Kloer, Severe hypertriglyceridemia and pancreatitis: presentation and management, Curr. Opin. Lipidol. 20 (2009) 497-504.

[385] J.R. Chora, A.C. Alves, A.M. Medeiros, C. Mariano, G. Lobarinhas, A. Guerra, H. Mansilha, H. Cortez-Pinto, M. Bourbon, Lysosomal acid lipase deficiency: a hidden disease among cohorts of familial hypercholesterolemia? J Clin Lipidol 11 (2017) 477-484.e2.

[386] B.K. Burton, M. Balwani, F. Feillet, I. Baric, T.A. Burrow, C. Camarena Grande, M. Coker, A. Consuelo-Sanchez, P. Deegan, M. Di Rocco, G.M. Enns, R. Erbe, F. Ezgu, C. Ficicioglu, K.N. Furuya, J. Kane, C. Laukaitis, E. Mengel, E.G. Neilan, S. Nightingale, H. Peters, M. Scarpa, K.O. Schwab, V. Smolka, V. Valayannopoulos, 
M. Wood, Z. Goodman, Y. Yang, S. Eckert, S. Rojas-Caro, A.G. Quinn, A phase 3 trial of sebelipase alfa in lysosomal acid lipase deficiency, N. Engl. J. Med. 373 (2015) 1010-1020.

[387] W. Hague, P. Forder, J. Simes, D. Hunt, A. Tonkin, LIPID Investigators. Effect of pravastatin on cardiovascular events and mortality in 1516 women with coronary heart disease: results from the Long-Term Intervention with Pravastatin in Ischemic Disease (LIPID) study, Am. Heart J. 145 (2003) 643-651.

[389] M.C. d'Emden, A.J. Jenkins, L. Li, D. Zannino, K.P. Mann, J.D. Best, B.G. Stuckey, K. Park, J. Saltevo, A.C. Keech, FIELD Study Investigators, Favourable effects of fenofibrate on lipids and cardiovascular disease in women with type 2 diabetes: results from the Fenofibrate Intervention and Event Lowering in Diabetes (FIELD) study, Diabetologia 57 (2014) 2296-2303.

[390] W.O. Spitzer, J.M. Faith, K.D. MacRae, Myocardial infarction and third generation oral contraceptives: aggregation of recent studies, Hum. Reprod. 17 (2002) 2307-2314.

[391] C.L. Shufelt, C.N. Bairey Merz, Contraceptive hormone use and cardiovascular disease, J. Am. Coll. Cardiol. 53 (2009) 221-231.

[392] J.E. Manson, J. Hsia, K.C. Johnson, J.E. Rossouw, A.R. Assaf, N.L. Lasser, M. Trevisan, H.R. Black, S.R. Heckbert, R. Detrano, O.L. Strickland, N.D. Wong, J.R. Crouse, E. Stein, M. Cushman, Women's Health Initiative Investigators. Estrogen plus progestin and the risk of coronary heart disease, N. Engl. J. Med. 349 (2003) 523-534.

[393] A. Rosengren, Better treatment and improved prognosis in elderly patients with AMI: but do registers tell the whole truth? Eur. Heart J. 33 (2012) 562-563.

[394] Second Joint Task Force of European and other Societies. Prevention of coronary heart disease in clinical practice. Recommendations of the Second Joint Task Force of European and other Societies on coronary prevention, Eur. Heart J. 19 (1998) 1434-1503.

[395] C. Koopman, I. Vaartjes, E.M. Heintjes, W. Spiering, I. van Dis, R.M. Herings, M.L. Bots, Persisting gender differences and attenuating age differences in cardiovascular drug use for prevention and treatment of coronary heart disease, 19982010, Eur. Heart J. 34 (2013) 3198-3205.

[396] J.A. Salami, H. Warraich, J. Valero-Elizondo, E.S. Spatz, N.R. Desai, J.S. Rana, S.S. Virani, R. Blankstein, A. Khera, M.J. Blaha, R.S. Blumenthal, D. Lloyd-Jones, K. Nasir, National trends in statin use and expenditures in the US adult population from 2002 to 2013: insights from the medical expenditure panel survey, JAMA Cardiol 2 (2017) 56-65.

[397] T.E. Strandberg, L. Kolehmainen, A. Vuorio, Evaluation and treatment of older patients with hypercholesterolemia: a clinical review, J. Am. Med. Assoc. 312 (2014) 1136-1144.

[398] D. Mangin, K. Sweeney, I. Heath, Preventive health care in elderly people needs rethinking, BMJ 335 (2007) 285-287.

[399] P.Z. Zimmet, Diabetes and its drivers: the largest epidemic in human history? Clin Diabetes Endocrinol 3 (2017) 1.

[400] A. Rawshani, A. Rawshani, S. Franzen, B. Eliasson, A.M. Svensson, M. Miftaraj, D.K. McGuire, N. Sattar, A. Rosengren, S. Gudbjornsdottir, Mortality and cardiovascular disease in type 1 and type 2 diabetes, N. Engl. J. Med. 376 (2017) 1407-1418.

[401] K.K.W. Olesen, M. Madsen, G. Egholm, T. Thim, L.O. Jensen, B. Raungaard, H.E. Botker, H.T. Sorensen, M. Maeng, Patients with diabetes without significant angiographic coronary artery disease have the same risk of myocardial infarction as patients without diabetes in a real-world population receiving appropriate prophylactic treatment, Diabetes Care 40 (2017) 1103-1110.

[402] Emerging Risk Factors Collaboration, N. Sarwar, P. Gao, S.R. Seshasai, R. Gobin, S. Kaptoge, E. Di Angelantonio, E. Ingelsson, D.A. Lawlor, E. Selvin, M. Stampfer, C.D. Stehouwer, S. Lewington, L. Pennells, A. Thompson, N. Sattar, I.R. White, K.K. Ray, J. Danesh, Diabetes mellitus, fasting blood glucose concentration, and risk of vascular disease: a collaborative meta-analysis of 102 prospective studies, Lancet 375 (2010) 2215-2222.

[403] E.W. Gregg, Y. Li, J. Wang, N.R. Burrows, M.K. Ali, D. Rolka, D.E. Williams, L. Geiss, Changes in diabetes-related complications in the United States, 19902010, N. Engl. J. Med. 370 (2014) 1514-1523.

[404] N. Sattar, Revisiting the links between glycaemia, diabetes and cardiovascular disease, Diabetologia 56 (2013) 686-695.

[405] J.R. Brownrigg, C.O. Hughes, D. Burleigh, A. Karthikesalingam, B.O. Patterson, P.J. Holt, M.M. Thompson, S. de Lusignan, K.K. Ray, R.J. Hinchliffe, Microvascular disease and risk of cardiovascular events among individuals with type 2 diabetes: a population-level cohort study, Lancet Diabetes Endocrinol 4 (2016) 588-597.

[406] J.P. Despres, Body fat distribution and risk of cardiovascular disease: an update, Circulation 126 (2012) 1301-1313.

[407] G. Targher, Non-alcoholic fatty liver disease as driving force in coronary heart disease? Gut 66 (2017) 213-214.

[408] G. Targher, A. Lonardo, C.D. Byrne, Nonalcoholic fatty liver disease and chronic vascular complications of diabetes mellitus, Nat. Rev. Endocrinol. 14 (2018) 99-114.

[409] S.M. Donahoe, G.C. Stewart, C.H. McCabe, S. Mohanavelu, S.A. Murphy, C.P. Cannon, E.M. Antman, Diabetes and mortality following acute coronary syndromes, J. Am. Med. Assoc. 298 (2007) 765-775.

[410] V. Levesque, P. Poirier, J.P. Despres, N. Almeras, Relation between a simple lifestyle risk score and established biological risk factors for cardiovascular disease, Am. J. Cardiol. 120 (2017) 1939-1946.

[411] M.R. Taskinen, J. Boren, New insights into the pathophysiology of dyslipidemia in type 2 diabetes, Atherosclerosis 239 (2015) 483-495.

[412] M.R. Taskinen, J. Boren, Why is apolipoprotein CIII emerging as a novel therapeutic target to reduce the burden of cardiovascular disease? Curr. Atheroscler. Rep. 18 (2016) 59.
[413] J. Boren, G.F. Watts, M. Adiels, S. Soderlund, D.C. Chan, A. Hakkarainen, N. Lundbom, N. Matikainen, J. Kahri, B. Verges, P.H. Barrett, M.R. Taskinen, Kinetic and related determinants of plasma triglyceride concentration in abdominal obesity: multicenter tracer kinetic study, Arterioscler. Thromb. Vasc. Biol. 35 (2015) 2218-2224.

[414] P.L. Gordts, R. Nock, N.H. Son, B. Ramms, I. Lew, J.C. Gonzales, B.E. Thacker D. Basu, R.G. Lee, A.E. Mullick, M.J. Graham, I.J. Goldberg, R.M. Crooke, J.L. Witztum, J.D. Esko, ApoC-III inhibits clearance of triglyceride-rich lipopro teins through LDL family receptors, J. Clin. Investig. 126 (2016) 2855-2866.

[415] L. Mark, A.J. Vallejo-Vaz, I. Reiber, G. Paragh, S.R. Kondapally Seshasai, K.K. Ray, Non-HDL cholesterol goal attainment and its relationship with triglyceride concentrations among diabetic subjects with cardiovascular disease: a nationwide survey of 2674 individuals in Hungary, Atherosclerosis 241 (2015) 62-68.

[416] R. Scott, R. O'Brien, G. Fulcher, C. Pardy, M. D'Emden, D. Tse, M.R. Taskinen, C. Ehnholm, A. Keech, Fenofibrate Intervention Event Lowering in Diabetes (FIELD) Study Investigators. Effects of fenofibrate treatment on cardiovascular disease risk in 9,795 individuals with type 2 diabetes and various components of the metabolic syndrome: the Fenofibrate Intervention and Event Lowering in Diabetes (FIELD) study, Diabetes Care 32 (2009) 493-498.

[417] G. Annuzzi, R. Giacco, L. Patti, L. Di Marino, C. De Natale, G. Costabile, M. Marra, C. Santangelo, R. Masella, A.A. Rivellese, Postprandial chylomicrons and adipose tissue lipoprotein lipase are altered in type 2 diabetes independently of obesity and whole-body insulin resistance, Nutr. Metab. Cardiovasc. Dis. 18 (2008) $531-538$.

[418] Cholesterol Treatment Trialists Collaboration, P.M. Kearney, L. Blackwell, R. Collins, A. Keech, J. Simes, R. Peto, J. Armitage, C. Baigent, Efficacy of cholesterol-lowering therapy in 18,686 people with diabetes in 14 randomised trials of statins: a meta-analysis, Lancet 371 (2008) 117-125.

[419] American Diabetes Association, Cardiovascular disease and risk management: standards of medical care in diabetes-2018, Diabetes Care 41 (2018) S86-S104.

[420] K.K. Ray, H.M. Colhoun, M. Szarek, M. Baccara-Dinet, D.L. Bhatt, V.A. Bittner, A.J. Budaj, R. Diaz, S.G. Goodman, C. Hanotin, R.A. Harrington, J.W. Jukema, V. Loizeau, R.D. Lopes, A. Moryusef, J. Murin, R. Pordy, A.D. Ristic, M.T. Roe, J. Tuñón, H.D. White, A.M. Zeiher, G.G. Schwartz, G. StegODYSSEY OUTCOMES Committees and Investigators, Effects of alirocumab on cardiovascular and metabolic outcomes after acute coronary syndrome in patients with or without diabetes: a prespecified analysis of the Odyssey outcomes randomised controlled trial, Lancet Diabetes Endocrinol (2019), https://doi.org/10.1016/S2213-8587(19) 30158-5 pii: S2213-8587(19)30158-5 [Epub ahead of print] Erratum in: Lancet Diabetes Endocrinol. 2019 July 8.

[421] M.B. Elam, H.N. Ginsberg, L.C. Lovato, M. Corson, J. Largay, L.A. Leiter, C. Lopez, P.J. O'Connor, M.E. Sweeney, D. Weiss, W.T. Friedewald, J.B. Buse, H.C. Gerstein, J. Probstfield, R. Grimm, F. Ismail-Beigi, D.C. Goff Jr., J.L. Fleg, Y. Rosenberg, R.P. ByingtonACCORDION Study Investigators, Association of fenofibrate therapy with long-term cardiovascular risk in statin-treated patients with type 2 diabetes, JAMA Cardiol 2 (2017) 370-380.

[422] S.A. Saha, R.R. Arora, Fibrates in the prevention of cardiovascular disease in patients with type 2 diabetes mellitus-a pooled meta-analysis of randomized placebo-controlled clinical trials, Int. J. Cardiol. 141 (2010) 157-166.

[423] E. Araki, S. Yamashita, H. Arai, K. Yokote, J. Satoh, T. Inoguchi, J. Nakamura, H. Maegawa, N. Yoshioka, Y. Tanizawa, H. Watada, H. Suganami, S. Ishibashi, Effects of pemafibrate, a novel selective PPARalpha modulator, on lipid and glucose metabolism in patients with type 2 diabetes and hypertriglyceridemia: a randomized, double-blind, placebo-controlled, phase 3 trial, Diabetes Care 41 (2018) 538-546.

[424] M. Arca, C. Borghi, R. Pontremoli, G.M. De Ferrari, F. Colivicchi, G. Desideri, P.L. Temporelli, Hypertriglyceridemia and omega-3 fatty acids: their often overlooked role in cardiovascular disease prevention, Nutr. Metab. Cardiovasc. Dis. 28 (2018) 197-205.

[425] D.L. Bhatt, P.G. Steg, E.A. Brinton, T.A. Jacobson, M. Miller, J.C. Tardif, S.B. Ketchum, R.T. Doyle Jr., S.A. Murphy, P.N. Soni, R.A. Braeckman, R.A. Juliano, C.M. BallantyneREDUCE-IT Investigators, Rationale and design of REDUCE-IT: Reduction of Cardiovascular Events with Icosapent Ethyl-Intervention Trial, Clin. Cardiol. 40 (2017) 138-148.

[426] ASCEND Study Collaborative Group, L. Bowman, M. Mafham, K. Wallendszus, W. Stevens, G. Buck, J. Barton, K. Murphy, T. Aung, R. Haynes, J. Cox, A. Murawska, A. Young, M. Lay, F. Chen, E. Sammons, E. Waters, A. Adler, J. Bodansky, A. Farmer, R. McPherson, A. Neil, D. Simpson, R. Peto, C. Baigent, R. Collins, S. Parish, J. Armitage, Effects of aspirin for primary prevention in persons with diabetes mellitus, N. Engl. J. Med. 379 (2018) 1529-1539.

[427] S.P. Laing, A.J. Swerdlow, S.D. Slater, A.C. Burden, A. Morris, N.R. Waugh, W. Gatling, P.J. Bingley, C.C. Patterson, Mortality from heart disease in a cohort of 23,000 patients with insulin-treated diabetes, Diabetologia 46 (2003) 760-765.

[428] J.J. Chillaron, J.A. Flores Le-Roux, D. Benaiges, J. Pedro-Botet, Type 1 diabetes, metabolic syndrome and cardiovascular risk, Metabolism 63 (2014) 181-187.

[429] G. Annuzzi, C. Iovine, B. Mandarino, L. Patti, L. Di Marino, G. Riccardi, A.A. Rivellese, Effect of acute exogenous hyperinsulinaemia on very low density lipoprotein subfraction composition in normal subjects, Eur. J. Clin. Investig. 31 (2001) 118-124.

[430] C.P. Cannon, B.A. Steinberg, S.A. Murphy, J.L. Mega, E. Braunwald, Meta-analysis of cardiovascular outcomes trials comparing intensive versus moderate statin therapy, J. Am. Coll. Cardiol. 48 (2006) 438-445.

[431] E.J. Mills, C. O'Regan, O. Eyawo, P. Wu, F. Mills, O. Berwanger, M. Briel, Intensive statin therapy compared with moderate dosing for prevention of cardiovascular events: a meta-analysis of > 40000 patients, Eur. Heart J. 32 (2011) 1409-1415.

[432] S.J. Nicholls, E.M. Tuzcu, S. Kalidindi, K. Wolski, K.W. Moon, I. Sipahi, 
P. Schoenhagen, S.E. Nissen, Effect of diabetes on progression of coronary atherosclerosis and arterial remodeling: a pooled analysis of 5 intravascular ultrasound trials, J. Am. Coll. Cardiol. 52 (2008) 255-262.

[433] D.G. Karalis, A.N. Hill, S. Clifton, R.A. Wild, The risks of statin use in pregnancy: a systematic review, J Clin Lipidol 10 (2016) 1081-1090.

[434] B. Gencer, K.C. Koskinas, L. Räber, A. Karagiannis, D. Nanchen, R. Auer, D. Carballo, S. Carballo, R. Klingenberg, D. Heg, C.M. Matter, T.F. Lüscher, N. Rodondi, F. Mach, S. Windecker, Eligibility for PCSK9 inhibitors according to American College of Cardiology (ACC) and European Society of Cardiology/ European Atherosclerosis Society (ESC/EAS) guidelines after acute coronary syndromes, J Am Heart Assoc 6 (2017) e006537.

[435] F. Kureshi, K.F. Kennedy, P.G. Jones, R.J. Thomas, S.V. Arnold, P. Sharma, T. Fendler, D.M. Buchanan, M. Qintar, P.M. Ho, B.K. Nallamothu, N.B. Oldridge, J.A. Spertus, Association between cardiac rehabilitation participation and health status outcomes after acute myocardial infarction, JAMA Cardiol 1 (2016) 980-988.

[436] K. Szummer, L. Wallentin, L. Lindhagen, J. Alfredsson, D. Erlinge, C. Held, S. James, T. Kellerth, B. Lindahl, A. Ravn-Fischer, E. Rydberg, T. Yndigegn, T. Jernberg, Improved outcomes in patients with ST-elevation myocardial infarction during the last 20 years are related to implementation of evidence-based treatments: experiences from the SWEDEHEART registry 1995-2014, Eur. Heart J. 38 (2017) 3056-3065.

[437] A.K. Gitt, D. Lautsch, J. Ferrieres, G.M. De Ferrari, A. Vyas, C.A. Baxter, L.D. Bash, V. Ashton, M. Horack, W. Almahmeed, F.T. Chiang, K.K. Poh, P. Brudi, B. Ambegaonkar, Cholesterol target value attainment and lipid-lowering therapy in patients with stable or acute coronary heart disease: results from the Dyslipidemia International Study II, Atherosclerosis 266 (2017) 158-166.

[438] J.A. de Lemos, M.A. Blazing, S.D. Wiviott, E.F. Lewis, K.A. Fox, H.D. White, J.L. Rouleau, T.R. Pedersen, L.H. Gardner, R. Mukherjee, K.E. Ramsey, J. Palmisano, D.W. Bilheimer, M.A. Pfeffer, R.M. Califf, E. Braunwaldfor the A to Z Investigators, Early intensive vs a delayed conservative simvastatin strategy in patients with acute coronary syndromes: phase Z of the A to Z trial, J. Am. Med. Assoc. 292 (2004) 1307-1316.

[439] E.P. Navarese, M. Kowalewski, F. Andreotti, M. van Wely, C. Camaro, M. Kolodziejczak, B. Gorny, J. Wirianta, J. Kubica, M. Kelm, M.J. de Boer, H. Suryapranata, Meta-analysis of time-related benefits of statin therapy in patients with acute coronary syndrome undergoing percutaneous coronary intervention, Am. J. Cardiol. 113 (2014) 1753-1764.

[440] K.K. Ray, C.P. Cannon, C.H. McCabe, R. Cairns, A.M. Tonkin, F.M. Sacks, G. Jackson, E. BraunwaldPROVE IT-TIMI 22 Investigators, Early and late benefits of high-dose atorvastatin in patients with acute coronary syndromes: results from the PROVE IT-TIMI 22 trial, J. Am. Coll. Cardiol. 46 (2005) 1405-1410.

[441] G.G. Schwartz, R. Fayyad, M. Szarek, D. DeMicco, A.G. Olsson, Early, intensive statin treatment reduces 'hard' cardiovascular outcomes after acute coronary syndrome, Eur J Prev Cardiol 24 (2017) 1294-1296.

[442] G.G. Schwartz, A.G. Olsson, M.D. Ezekowitz, P. Ganz, M.F. Oliver, D. Waters, A. Zeiher, B.R. Chaitman, S. Leslie, T. SternMyocardial Ischemia Reduction with Aggressive Cholesterol Lowering (MIRACL) Study Investigators, Effects of atorvastatin on early recurrent ischemic events in acute coronary syndromes: the MIRACL study: a randomized controlled trial, J. Am. Med. Assoc. 285 (2001) 1711-1718.

[443] O. Berwanger, E.V. Santucci, E.S.P.G.M. de Barros, I.A. Jesuino, L.P. Damiani, L.M. Barbosa, R.H.N. Santos, L.N. Laranjeira, F.M. Egydio, J.A. Borges de Oliveira, F.T.C. Dall Orto, P. Beraldo de Andrade, I.R.C. Bienert, C.E. Bosso, J.A. Mangione, C.A. Polanczyk, A. Sousa, R.A.K. Kalil, L.M. Santos, A.C. Sposito, R.L. Rech, A.C.S. Sousa, F. Baldissera, B.R. Nascimento, R. Giraldez, A.B. Cavalcanti, S.B. Pereira, L.A. Mattos, L.V. Armaganijan, H.P. Guimaraes, J. Sousa, J.H. Alexander, C.B. Granger, R.D. LopesSECURE-PCI Investigators, Effect of loading dose of atorvastatin prior to planned percutaneous coronary intervention on major adverse cardiovascular events in acute coronary syndrome: the SECUREPCI randomized clinical trial, J. Am. Med. Assoc. 319 (2018) 1331-1340.

[444] E.T. Kato, C.P. Cannon, M.A. Blazing, E. Bohula, S. Guneri, J.A. White, S.A. Murphy, J.G. Park, E. Braunwald, R.P. Giugliano, Efficacy and safety of adding ezetimibe to statin therapy among women and men: insight from IMPROVE-IT (Improved Reduction of Outcomes: Vytorin Efficacy International Trial), J Am Heart Assoc 6 (2017) e006901.

[445] S.A. Murphy, C.P. Cannon, M.A. Blazing, R.P. Giugliano, J.A. White, Y. Lokhnygina, C. Reist, K. Im, E.A. Bohula, D. Isaza, J. Lopez-Sendon, M. Dellborg, U. Kher, A.M. Tershakovec, E. Braunwald, Reduction in total cardiovascular events with ezetimibe/simvastatin post-acute coronary syndrome: the IMPROVE-IT trial, J. Am. Coll. Cardiol. 67 (2016) 353-361.

[446] E.A. Bohula, S.D. Wiviott, R.P. Giugliano, M.A. Blazing, J.G. Park, S.A. Murphy, J.A. White, F. Mach, F. Van de Werf, A.J. Dalby, H.D. White, A.M. Tershakovec, C.P. Cannon, E. Braunwald, Prevention of stroke with the addition of ezetimibe to statin therapy in patients with acute coronary syndrome in IMPROVE-IT (Improved Reduction of Outcomes: Vytorin Efficacy International Trial), Circulation 136 (2017) 2440-2450.

[447] Y. Pokharel, K. Chinnakondepalli, K. Vilain, K. Wang, D.B. Mark, G. Davies, M.A. Blazing, R.P. Giugliano, E. Braunwald, C.P. Cannon, D.J. Cohen, E.A. Magnuson, Impact of ezetimibe on the rate of cardiovascular-related hospitalizations and associated costs among patients with a recent acute coronary syndrome: results from the IMPROVE-IT trial (Improved Reduction of Outcomes: Vytorin Efficacy International Trial), Circ Cardiovasc Qual Outcomes 10 (2017) e003201.

[448] E.A. Bohula, D.A. Morrow, R.P. Giugliano, M.A. Blazing, P. He, J.G. Park, S.A. Murphy, J.A. White, Y.A. Kesaniemi, T.R. Pedersen, A.J. Brady, Y. Mitchel,
C.P. Cannon, E. Braunwald, Atherothrombotic risk stratification and ezetimibe for secondary prevention, J. Am. Coll. Cardiol. 69 (2017) 911-921.

[449] N. Hagiwara, E. Kawada-Watanabe, R. Koyanagi, H. Arashi, J. Yamaguchi, K. Nakao, T. Tobaru, H. Tanaka, T. Oka, Y. Endoh, K. Saito, T. Uchida, K. Matsui, H. Ogawa, Low-density lipoprotein cholesterol targeting with pitavastatin + ezetimibe for patients with acute coronary syndrome and dyslipidaemia: the HIJPROPER study, a prospective, open-label, randomized trial, Eur. Heart J. 38 (2017) 2264-2276.

[450] M.S. Sabatine, G.M. De Ferrari, R.P. Giugliano, K. Huber, B.S. Lewis, J. Ferreira, J.F. Kuder, S.A. Murphy, S.D. Wiviott, C.E. Kurtz, N. Honarpour, A.C. Keech, P.S. Sever, T.R. Pedersen, Clinical benefit of evolocumab by severity and extent of coronary artery disease, Circulation 138 (2018) 756-766.

[451] R.P. Giugliano, T.R. Pedersen, J.G. Park, G.M. De Ferrari, Z.A. Gaciong, R. Ceska, K. Toth, I. Gouni-Berthold, J. Lopez-Miranda, F. Schiele, F. Mach, B.R. Ott, E. Kanevsky, A.L. Pineda, R. Somaratne, S.M. Wasserman, A.C. Keech, P.S. Sever, M.S. SabatineFOURIER Investigators, Clinical efficacy and safety of achieving very low LDL-cholesterol concentrations with the PCSK9 inhibitor evolocumab: a prespecified secondary analysis of the FOURIER trial, Lancet 390 (2017) 1962-1971.

[452] K.C. Koskinas, S. Windecker, A. Buhayer, B. Gencer, G. Pedrazzini, C. Mueller, S. Cook, O. Muller, C.M. Matter, L. Raber, D. Heg, F. MachEVOPACS Investigators, Design of the randomized, placebo-controlled evolocumab for early reduction of LDL-cholesterol levels in patients with acute coronary syndromes (EVOPACS) trial, Clin. Cardiol. 41 (2018) 1513-1520.

[453] T. Aung, J. Halsey, D. Kromhout, H.C. Gerstein, R. Marchioli, L. Tavazzi, J.M. Geleijnse, B. Rauch, A. Ness, P. Galan, E.Y. Chew, J. Bosch, R. Collins, S. Lewington, J. Armitage, R. Clarke, Omega-3 Treatment Trialists' Collaboration. Associations of omega-3 fatty acid supplement use with cardiovascular disease risks: meta-analysis of 10 trials involving 77917 individuals, JAMA Cardiol 3 (2018) 225-234.

[454] G. Patti, C.P. Cannon, S.A. Murphy, S. Mega, V. Pasceri, C. Briguori, A. Colombo, K.H. Yun, M.H. Jeong, J.S. Kim, D. Choi, H. Bozbas, M. Kinoshita, K. Fukuda, X.W. Jia, H. Hara, S. Cay, G. Di Sciascio, Clinical benefit of statin pretreatment in patients undergoing percutaneous coronary intervention: a collaborative patientlevel meta-analysis of 13 randomized studies, Circulation 123 (2011) 1622-1632.

[455] J.S. Kim, J. Kim, D. Choi, C.J. Lee, S.H. Lee, Y.G. Ko, M.K. Hong, B.K. Kim, S.J. Oh, D.W. Jeon, J.Y. Yang, J.R. Cho, N.H. Lee, Y.H. Cho, D.K. Cho, Y. Jang, Efficacy of high-dose atorvastatin loading before primary percutaneous coronary intervention in ST-segment elevation myocardial infarction: the STATIN STEMI trial, JACC Cardiovasc. Interv. 3 (2010) 332-339.

[456] C. Briguori, G. Visconti, A. Focaccio, B. Golia, A. Chieffo, A. Castelli, M. Mussardo, M. Montorfano, B. Ricciardelli, A. Colombo, Novel approaches for preventing or limiting events (Naples) II trial: impact of a single high loading dose of atorvastatin on periprocedural myocardial infarction, J. Am. Coll. Cardiol. 54 (2009) $2157-2163$.

[457] G. Di Sciascio, G. Patti, V. Pasceri, A. Gaspardone, G. Colonna, A. Montinaro, Efficacy of atorvastatin reload in patients on chronic statin therapy undergoing percutaneous coronary intervention: results of the ARMYDA-RECAPTURE (Atorvastatin for Reduction of Myocardial Damage During Angioplasty) randomized trial, J. Am. Coll. Cardiol. 54 (2009) 558-565.

[458] E.P. Navarese, P.A. Gurbel, F. Andreotti, M.M. Kołodziejczak, S.C. Palmer, S. Dias, A. Buffon, J. Kubica, M. Kowalewski, T. Jadczyk, M. Laskiewicz, M. Jedrzejek, M. Brockmeyer, F. Airoldi, M. Ruospo, S. De Servi, W. Wojakowski, C. O'Connor, G.F. Strippoli, Prevention of contrast-induced acute kidney injury in patients undergoing cardiovascular procedures-a systematic review and network meta-analysis, PLoS One 12 (2017) e0168726.

[459] P. Amarenco, J. Bogousslavsky, A. Callahan III, L.B. Goldstein, M. Hennerici, A.E. Rudolph, H. Sillesen, L. Simunovic, M. Szarek, K.M. Welch, J.A. ZivinStroke Prevention by Aggressive Reduction in Cholesterol Levels (SPARCL) Investigators, High-dose atorvastatin after stroke or transient ischemic attack, N. Engl. J. Med. 355 (2006) 549-559.

[460] P. Amarenco, J. Labreuche, Lipid management in the prevention of stroke: review and updated meta-analysis of statins for stroke prevention, Lancet Neurol. 8 (2009) 453-463.

[461] A. Merwick, G.W. Albers, E.M. Arsava, H. Ay, D. Calvet, S.B. Coutts, B.L. Cucchiara, A.M. Demchuk, M.F. Giles, J.L. Mas, J.M. Olivot, F. Purroy, P.M. Rothwell, J.L. Saver, V.K. Sharma, G. Tsivgoulis, P.J. Kelly, Reduction in early stroke risk in carotid stenosis with transient ischemic attack associated with statin treatment, Stroke 44 (2013) 2814-2820.

[462] A.C. Flint, C. Conell, X. Ren, H. Kamel, S.L. Chan, V.A. Rao, S.C. Johnston, Statin adherence is associated with reduced recurrent stroke risk in patients with or without atrial fibrillation, Stroke 48 (2017) 1788-1794.

[463] The Long-Term Intervention with Pravastatin in Ischemic Disease (LIPID) Study Group. Prevention of cardiovascular events and death with pravastatin in patients with coronary heart disease and a broad range of initial cholesterol levels. The Long-Term Intervention with Pravastatin in Ischaemic Disease (LIPID) Study Group, N. Engl. J. Med. 339 (1998) 1349-1357.

[464] J. Kjekshus, T.R. Pedersen, A.G. Olsson, O. Faergeman, K. Pyorala, The effects of simvastatin on the incidence of heart failure in patients with coronary heart disease, J. Card. Fail. 3 (1997) 249-254.

[465] K.K. Khush, D.D. Waters, V. Bittner, P.C. Deedwania, J.J. Kastelein, S.J. Lewis, N.K. Wenger, Effect of high-dose atorvastatin on hospitalizations for heart failure: subgroup analysis of the Treating to New Targets (TNT) study, Circulation 115 (2007) 576-583.

[466] J. Kjekshus, E. Apetrei, V. Barrios, M. Böhm, J.G. Cleland, J.H. Cornel, P. Dunselman, C. Fonseca, A. Goudev, P. Grande, L. Gullestad, A. Hjalmarson, J. Hradec, A. Jánosi, G. Kamenský, M. Komajda, J. Korewicki, T. Kuusi, F. Mach, 
V. Mareev, J.J. McMurray, N. Ranjith, M. Schaufelberger, J. Vanhaecke, D.J. van Veldhuisen, F. Waagstein, H. Wedel, J. Wikstrand, CORONA Group, Rosuvastatin in older patients with systolic heart failure, N. Engl. J. Med. 357 (2007) 2248-2261.

[467] F.M. Sacks, M.A. Pfeffer, L.A. Moye, J.L. Rouleau, J.D. Rutherford, T.G. Cole, L. Brown, J.W. Warnica, J.M. Arnold, C.C. Wun, B.R. Davis, E. Braunwald, The effect of pravastatin on coronary events after myocardial infarction in patients with average cholesterol levels. Cholesterol and Recurrent Events Trial investigators, N. Engl. J. Med. 335 (1996) 1001-1009.

[468] B.M. Scirica, D.A. Morrow, C.P. Cannon, K.K. Ray, M.S. Sabatine, P. Jarolim, A. Shui, C.H. McCabe, E. BraunwaldPROVE IT-TIMI 22 Investigators, Intensive statin therapy and the risk of hospitalization for heart failure after an acute coronary syndrome in the PROVE IT-TIMI 22 study, J. Am. Coll. Cardiol. 47 (2006) $2326-2331$.

[469] D. Preiss, R.T. Campbell, H.M. Murray, I. Ford, C.J. Packard, N. Sattar, K. Rahimi, H.M. Colhoun, D.D. Waters, J.C. LaRosa, P. Amarenco, T.R. Pedersen, M.J. Tikkanen, M.J. Koren, N.R. Poulter, P.S. Sever, P.M. Ridker, J.G. MacFadyen, S.D. Solomon, B.R. Davis, L.M. Simpson, H. Nakamura, K. Mizuno, R.M. Marfisi, R. Marchioli, G. Tognoni, V.G. Athyros, K.K. Ray, A.M. Gotto, M.B. Clearfield, J.R. Downs, J.J. McMurray, The effect of statin therapy on heart failure events: a collaborative meta-analysis of unpublished data from major randomized trials, Eur. Heart J. 36 (2015) 1536-1546.

[470] L. Tavazzi, A.P. Maggioni, R. Marchioli, S. Barlera, M.G. Franzosi, R. Latini, D. Lucci, G.L. Nicolosi, M. Porcu, G. TognoniGISSI-HF Investigators, Effect of rosuvastatin in patients with chronic heart failure (the GISSI-HF trial): a randomised, double-blind, placebo-controlled trial, Lancet 372 (2008) 1231-1239.

[471] M. Al-Gobari, H.H. Le, M. Fall, F. Gueyffier, B. Burnand, No benefits of statins for sudden cardiac death prevention in patients with heart failure and reduced ejection fraction: a meta-analysis of randomized controlled trials, PLoS One 12 (2017) e0171168.

[472] M.J. Feinstein, P. Jhund, J. Kang, H. Ning, A. Maggioni, J. Wikstrand, J. Kjekshus, L. Tavazzi, J. McMurray, D.M. Lloyd-Jones, Do statins reduce the risk of myocardial infarction in patients with heart failure? A pooled individual-level reanalysis of CORONA and GISSI-HF, Eur. J. Heart Fail. 17 (2015) 434-441.

[473] A. Bayes-Genis, J. Nunez, F. Zannad, J.P. Ferreira, S.D. Anker, J.G. Cleland, K. Dickstein, G. Filippatos, C.C. Lang, L.L. Ng, P. Ponikowski, N.J. Samani, D.J. van Veldhuisen, A.H. Zwinderman, M. Metra, J. Lupon, A.A. Voors, The PCSK9-LDI receptor axis and outcomes in heart failure: BIOSTAT-CHF subanalysis, J. Am. Coll. Cardiol. 70 (2017) 2128-2136.

[474] G.S. Francis, Cholesterol and heart failure: is there an important connection? J. Am. Coll. Cardiol. 70 (2017) 2137-2138.

[475] L. Tavazzi, A.P. Maggioni, R. Marchioli, S. Barlera, M.G. Franzosi, R. Latini, D. Lucci, G.L. Nicolosi, M. Porcu, G. TognoniGISSI-HF Investigators, Effect of n-3 polyunsaturated fatty acids in patients with chronic heart failure (the GISSI-HF trial): a randomised, double-blind, placebo-controlled trial, Lancet 372 (2008) 1223-1230.

[476] J.G. Smith, K. Luk, C.A. Schulz, J.C. Engert, R. Do, G. Hindy, G. Rukh, L. Dufresne, P. Almgren, D.S. Owens, T. B. Harris, G.M. Peloso, K. F. Kerr, Q. Wong, A.V. Smith, M.J. Budoff, J.I. Rotter, L.A. Cupples, S. Rich, S. Kathiresan, M. Orho-Melander, V. Gudnason, C.J. O'Donnell, W.S. Post, G. Thanassoulis, Cohorts for Heart and Aging Research in Genetic Epidemiology (CGARGE) Extracoronary Calcium Working Group. Association of low-density lipoprotein cholesterol-related genetic variants with aortic valve calcium and incident aortic stenosis, J. Am. Med. Assoc. 312 (2014) 1764-1771.

[477] S. Tsimikas, A test in context: lipoprotein(a): diagnosis, prognosis, controversies, and emerging therapies, J. Am. Coll. Cardiol. 69 (2017) 692-711.

[478] G.M. Novaro, I.Y. Tiong, G.L. Pearce, M.S. Lauer, D.L. Sprecher, B.P. Griffin, Effect of hydroxymethylglutaryl coenzyme a reductase inhibitors on the progression of calcific aortic stenosis, Circulation 104 (2001) 2205-2209.

[479] K.L. Chan, K. Teo, J.G. Dumesnil, A. Ni, J. TamASTRONOMER Investigators, Effect of Lipid lowering with rosuvastatin on progression of aortic stenosis: results of the aortic stenosis progression observation: measuring effects of rosuvastatin (ASTRONOMER) trial, Circulation 121 (2010) 306-314.

[480] S.J. Cowell, D.E. Newby, R.J. Prescott, P. Bloomfield, J. Reid, D.B. Northridge, N.A. BoonScottish Aortic Stenosis and Lipid Lowering Trial, Impact on Regression (SALTIRE) Investigators, A randomized trial of intensive lipid-lowering therapy in calcific aortic stenosis, N. Engl. J. Med. 352 (2005) 2389-2397.

[481] W. Dichtl, H.F. Alber, G.M. Feuchtner, F. Hintringer, M. Reinthaler, T. Bartel, A. Sussenbacher, W. Grander, H. Ulmer, O. Pachinger, S. Muller, Prognosis and risk factors in patients with asymptomatic aortic stenosis and their modulation by atorvastatin (20 mg), Am. J. Cardiol. 102 (2008) 743-748.

[482] L. Thiago, S.R. Tsuji, J. Nyong, M.E. Puga, A.F. Gois, C.R. Macedo, O. Valente, A.N. Atallah, Statins for aortic valve stenosis, Cochrane Database Syst. Rev. 9 (2016) CD009571.

[483] Y. Zhao, R. Nicoll, Y.H. He, M.Y. Henein, The effect of statins on valve function and calcification in aortic stenosis: a meta-analysis, Atherosclerosis 246 (2016) 318-324.

[484] A.M. Greve, C.N. Bang, K. Boman, K. Egstrup, J.L. Forman, Y.A. Kesaniemi, S. Ray, T.R. Pedersen, P. Best, N.M. Rajamannan, K. Wachtell, Effect modifications of lipid-lowering therapy on progression of aortic stenosis (from the Simvastatin and Ezetimibe in Aortic Stenosis [SEAS] Study), Am. J. Cardiol. 121 (2018) 739-745.

[485] B.J. Arsenault, S.M. Boekholdt, S. Mora, D.A. DeMicco, W. Bao, J.C. Tardif, P. Amarenco, T. Pedersen, P. Barter, D.D. Waters, Impact of high-dose atorvastatin therapy and clinical risk factors on incident aortic valve stenosis in patients with cardiovascular disease (from TNT, IDEAL, and SPARCL), Am. J. Cardiol. 113 (2014) 1378-1382.
[486] C.P. Huded, L.R. Benck, N.J. Stone, R.N. Sweis, M.J. Ricciardi, S.C. Malaisrie, C.J. Davidson, J.D. Flaherty, Relation of intensity of statin therapy and outcomes after transcatheter aortic valve replacement, Am. J. Cardiol. 119 (2017) $1832-1838$

[487] A.C. Milin, G. Vorobiof, O. Aksoy, R. Ardehali, Insights into aortic sclerosis and its relationship with coronary artery disease, J Am Heart Assoc 3 (2014) e001111.

[488] P.E. Stevens, A. Levin, Kidney Disease, Improving Global Outcomes Chronic Kidney Disease Guideline Development Work Group Members. Evaluation and management of chronic kidney disease: synopsis of the kidney disease: improving global outcomes 2012 clinical practice guideline, Ann. Intern. Med. 158 (2013) 825-830.

[489] B. Franczyk-Skora, A. Gluba, M. Banach, P. Rozentryt, L. Polonski, J. Rysz, Acute coronary syndromes in patients with chronic kidney disease, Curr. Vasc. Pharmacol. 11 (2013) 758-767.

[490] R.T. Gansevoort, R. Correa-Rotter, B.R. Hemmelgarn, T.H. Jafar, H.J. Heerspink, J.F. Mann, K. Matsushita, C.P. Wen, Chronic kidney disease and cardiovascular risk: epidemiology, mechanisms, and prevention, Lancet 382 (2013) 339-352.

[491] S. Olechnowicz-Tietz, A. Gluba, A. Paradowska, M. Banach, J. Rysz, The risk of atherosclerosis in patients with chronic kidney disease, Int. Urol. Nephrol. 45 (2013) 1605-1612.

[492] K. Chronic Kidney Disease Prognosis ConsortiumMatsushita, M. van der Velde, B.C. Astor, M. Woodward, A.S. Levey, P.E. de Jong, J. Coresh, R.T. Gansevoort, Association of estimated glomerular filtration rate and albuminuria with all-cause and cardiovascular mortality in general population cohorts: a collaborative metaanalysis, Lancet 375 (2010) 2073-2081.

[493] G. Loncar, O. Barthelemy, E. Berman, M. Kerneis, T. Petroni, L. Payot, R. Choussat, J. Silvain, J.P. Collet, G. Helft, G. Montalescot, C. Le Feuvre, Impact of renal failure on all-cause mortality and other outcomes in patients treated by percutaneous coronary intervention, Arch Cardiovasc Dis 108 (2015) 554-562.

[494] M. Tonelli, C. Wanner, Kidney Disease, Improving Global Outcomes Lipid Guideline Development Work Group Members. Lipid management in chronic kidney disease: synopsis of the Kidney Disease: Improving Global Outcomes 2013 clinical practice guideline, Ann. Intern. Med. 160 (2014) 182.

[497] A. Agarwal, G.V. Prasad, Post-transplant dyslipidemia: mechanisms, diagnosis and management, World J. Transplant. 6 (2016) 125-134.

[498] O. Bamgbola, Metabolic consequences of modern immunosuppressive agents in solid organ transplantation, Ther Adv Endocrinol Metab 7 (2016) 110-127.

[499] K. Numakura, H. Kagaya, R. Yamamoto, N. Komine, M. Saito, T. Hiroshi, S. Akihama, T. Inoue, S. Narita, N. Tsuchiya, T. Habuchi, T. Niioka, M. Miura, S. Satoh, Characterization of clinical and genetic risk factors associated with dyslipidemia after kidney transplantation, Dis. Markers 2015 (2015) 179434.

[500] A.S. Pinto, M.F. Chedid, L.T. Guerra, D.D. Cabeleira, C.D. Kruel, Dietary management for dyslipidemia in liver transplant recipients, Arq Bras Cir Dig 29 (2016) $246-251$

[501] L.V. Riella, S. Gabardi, A. Chandraker, Dyslipidemia and its therapeutic challenges in renal transplantation, Am. J. Transplant. 12 (2012) 1975-1982.

[502] K. Claes, H.U. Meier-Kriesche, J.D. Schold, Y. Vanrenterghem, P.F. Halloran, H. Ekberg, Effect of different immunosuppressive regimens on the evolution of distinct metabolic parameters: evidence from the Symphony study, Nephrol. Dial. Transplant. 27 (2012) 850-857.

[503] S. Deleuze, V. Garrigue, S. Delmas, G. Chong, I. Swarcz, J.P. Cristol, G. Mourad, New onset dyslipidemia after renal transplantation: is there a difference between tacrolimus and cyclosporine? Transplant. Proc. 38 (2006) 2311-2313.

[504] B.L. Kasiske, A. de Mattos, S.M. Flechner, L. Gallon, H.U. Meier-Kriesche, M.R. Weir, A. Wilkinson, Mammalian target of rapamycin inhibitor dyslipidemia in kidney transplant recipients, Am. J. Transplant. 8 (2008) 1384-1392.

[505] H.Y. Li, B. Li, Y.G. Wei, L.N. Yan, T.F. Wen, J.C. Zhao, M.Q. Xu, W.T. Wang, Y.K. Ma, J.Y. Yang, Higher tacrolimus blood concentration is related to hyperlipidemia in living donor liver transplantation recipients, Dig. Dis. Sci. 57 (2012) 204-209.

[506] J.D. Morrisett, G. Abdel-Fattah, R. Hoogeveen, E. Mitchell, C.M. Ballantyne, H.J. Pownall, A.R. Opekun, J.S. Jaffe, S. Oppermann, B.D. Kahan, Effects of sirolimus on plasma lipids, lipoprotein levels, and fatty acid metabolism in renal transplant patients, J. Lipid Res. 43 (2002) 1170-1180.

[507] R.L. Page II, G.G. Miller, J. Lindenfeld, Drug therapy in the heart transplant recipient: part IV: drug-drug interactions, Circulation 111 (2005) 230-239.

[508] F. Almutairi, T.C. Peterson, M. Molinari, M.J. Walsh, I. Alwayn, K.M. Peltekian, Safety and effectiveness of ezetimibe in liver transplant recipients with hypercholesterolemia, Liver Transplant. 15 (2009) 504-508.

[509] S.M. Shaw, P. Chaggar, J. Ritchie, M.K. Shah, A.C. Baynes, N. O'Neill, J.E. Fildes, N. Yonan, S.G. Williams, The efficacy and tolerability of ezetimibe in cardiac transplant recipients taking cyclosporin, Transplantation 87 (2009) 771-775.

[510] M. European Stroke OrganisationTendera, V. Aboyans, M.L. Bartelink, I. Baumgartner, D. Clement, J.P. Collet, A. Cremonesi, M. De Carlo, R. Erbel, F.G. Fowkes, M. Heras, S. Kownator, E. Minar, J. Ostergren, D. Poldermans, V. Riambau, M. Roffi, J. Rother, H. Sievert, M. van Sambeek, T. Zeller, ESC Committee for Practice Guidelines, ESC Guidelines on the diagnosis and treatment of peripheral artery diseases: Document covering atherosclerotic disease of extracranial carotid and vertebral, mesenteric, renal, upper and lower extremity arteries: the Task Force on the Diagnosis and Treatment of Peripheral Artery Diseases of the European Society of Cardiology (ESC), Eur. Heart J. 32 (2011) 2851-2906.

[511] M.M. McDermott, A.L. Mandapat, A. Moates, M. Albay, E. Chiou, L. Celic, P. Greenland, Knowledge and attitudes regarding cardiovascular disease risk and prevention in patients with coronary or peripheral arterial disease, Arch. Intern. Med. 163 (2003) 2157-2162. 
[512] P.P. Aung, H.G. Maxwell, R.G. Jepson, J.F. Price, G.C. Leng, Lipid-lowering for peripheral arterial disease of the lower limb, Cochrane Database Syst. Rev. 4 (2007) CD000123.

[513] R. Collins, J. Armitage, S. Parish, P. Sleigh, R. Peto, Heart Protection Study Collaborative Group, MRC/BHF Heart Protection Study of cholesterol-lowering with simvastatin in 5963 people with diabetes: a randomised placebo-controlled trial, Lancet 361 (2003) 2005-2016.

[514] M.P. Bonaca, P. Nault, R.P. Giugliano, A.C. Keech, A.L. Pineda, E. Kanevsky, J. Kuder, S.A. Murphy, J.W. Jukema, B.S. Lewis, L. Tokgozoglu, R. Somaratne, P.S. Sever, T.R. Pedersen, M.S. Sabatine, Low-density lipoprotein cholesterol lowering with evolocumab and outcomes in patients with peripheral artery disease: insights from the FOURIER trial (Further Cardiovascular Outcomes Research With PCSK9 Inhibition in Subjects With Elevated Risk), Circulation 137 (2018) 338-350.

[515] K. Rajamani, P.G. Colman, L.P. Li, J.D. Best, M. Voysey, M.C. D'Emden, M. Laakso, J.R. Baker, A.C. KeechFIELD study investigators, Effect of fenofibrate on amputation events in people with type 2 diabetes mellitus (FIELD study): a prespecified analysis of a randomised controlled trial, Lancet 373 (2009) 1780-1788.

[516] E. Tedeschi-Reiner, M. Strozzi, B. Skoric, Z. Reiner, Relation of atherosclerotic changes in retinal arteries to the extent of coronary artery disease, Am. J. Cardiol. 96 (2005) 1107-1109.

[517] Accord Study Group, Accord Eye Study Group, E.Y. Chew, W.T. Ambrosius, M.D. Davis, R.P. Danis, S. Gangaputra, C.M. Greven, L. Hubbard, B.A. Esser, J.F. Lovato, L.H. Perdue, D.C. Goff Jr., W.C. Cushman, H.N. Ginsberg, M.B. Elam, S. Genuth, H.C. Gerstein, U. Schubart, L.J. Fine, Effects of medical therapies on retinopathy progression in type 2 diabetes, N. Engl. J. Med. 363 (2010) 233-244.

[518] A.C. Keech, P. Mitchell, P.A. Summanen, J. O'Day, T.M. Davis, M.S. Moffitt, M.R. Taskinen, R.J. Simes, D. Tse, E. Williamson, A. Merrifield, L.T. Laatikainen, M.C. d'Emden, D.C. Crimet, R.L. O'Connell, P.G. ColmanFIELD study investigators, Effect of fenofibrate on the need for laser treatment for diabetic retinopathy (FIELD study): a randomised controlled trial, Lancet 370 (2007) 1687-1697.

[519] J. Golledge, J. Muller, A. Daugherty, P. Norman, Abdominal aortic aneurysm: pathogenesis and implications for management, Arterioscler. Thromb. Vasc. Biol. 26 (2006) 2605-2613.

[520] K.I. Paraskevas, C.D. Liapis, G. Hamilton, D.P. Mikhailidis, Can statins reduce perioperative morbidity and mortality in patients undergoing non-cardiac vascular surgery? Eur. J. Vasc. Endovasc. Surg. 32 (2006) 286-293.

[521] A.E. Durazzo, F.S. Machado, D.T. Ikeoka, C. De Bernoche, M.C. Monachini, P. Puech-Leao, B. Caramelli, Reduction in cardiovascular events after vascular surgery with atorvastatin: a randomized trial, J. Vasc. Surg. 39 (2004) 967-975 discussion 975-976.

[522] O. Schouten, E. Boersma, S.E. Hoeks, R. Benner, H. van Urk, M.R. van Sambeek, H.J. Verhagen, N.A. Khan, M. Dunkelgrun, J.J. Bax, D. Poldermans, Dutch Echocardiographic Cardiac Risk Evaluation Applying Stress Echocardiography Study Group. Fluvastatin and perioperative events in patients undergoing vascular surgery, N. Engl. J. Med. 361 (2009) 980-989.

[523] D.G. Hackam, F. Wu, P. Li, P.C. Austin, S.W. Tobe, M.M. Mamdani, A.X. Garg, Statins and renovascular disease in the elderly: a population-based cohort study, Eur. Heart J. 32 (2011) 598-610.

[525] R. Ross, Atherosclerosis-an inflammatory disease, N. Engl. J. Med. 340 (1999) $115-126$.

[526] I. Tabas, A.H. Lichtman, Monocyte-macrophages and T cells in atherosclerosis, Immunity 47 (2017) 621-634.

[527] B. Emini Veseli, P. Perrotta, G.R.A. De Meyer, L. Roth, C. Van der Donckt, W. Martinet, G.R.Y. De Meyer, Animal models of atherosclerosis, Eur. J. Pharmacol. 816 (2017) 3-13.

[528] W.S. Tillett, T. Francis, Serological reactions in pneumonia with a non-protein somatic fraction of Pneumococcus, J. Exp. Med. 52 (1930) 561-571.

[529] M.S. Sabatine, D.A. Morrow, K.A. Jablonski, M.M. Rice, J.W. Warnica, M.J. Domanski, J. Hsia, B.J. Gersh, N. Rifai, P.M. Ridker, M.A. Pfeffer, E. BraunwaldPEACE Investigators, Prognostic significance of the Centers for Disease Control/American Heart Association high-sensitivity C-reactive protein cut points for cardiovascular and other outcomes in patients with stable coronary artery disease, Circulation 115 (2007) 1528-1536.

[530] P.M. Ridker, M. Cushman, M.J. Stampfer, R.P. Tracy, C.H. Hennekens, Inflammation, aspirin, and the risk of cardiovascular disease in apparently healthy men, N. Engl. J. Med. 336 (1997) 973-979.

[531] P.M. Ridker, C.H. Hennekens, J.E. Buring, N. Rifai, C-reactive protein and other markers of inflammation in the prediction of cardiovascular disease in women, $\mathrm{N}$. Engl. J. Med. 342 (2000) 836-843.

[532] J. Danesh, J.G. Wheeler, G.M. Hirschfield, S. Eda, G. Eiriksdottir, A. Rumley, G.D. Lowe, M.B. Pepys, V. Gudnason, C-reactive protein and other circulating markers of inflammation in the prediction of coronary heart disease, N. Engl. J. Med. 350 (2004) 1387-1397.

[533] P. Elliott, J.C. Chambers, W. Zhang, R. Clarke, J.C. Hopewell, J.F. Peden, J. Erdmann, P. Braund, J.C. Engert, D. Bennett, L. Coin, D. Ashby, I. Tzoulaki, I.J. Brown, S. Mt-Isa, M.I. McCarthy, L. Peltonen, N.B. Freimer, M. Farrall, A. Ruokonen, A. Hamsten, N. Lim, P. Froguel, D.M. Waterworth, P. Vollenweider, G. Waeber, M.R. Jarvelin, V. Mooser, J. Scott, A.S. Hall, H. Schunkert, S.S. Anand, R. Collins, N.J. Samani, H. Watkins, J.S. Kooner, Genetic loci associated with Creactive protein levels and risk of coronary heart disease, J. Am. Med. Assoc. 302 (2009) 37-48.

[534] Emerging Risk Factors Collaboration, S. Kaptoge, E. Di Angelantonio, L. Pennells, A.M. Wood, I.R. White, P. Gao, M. Walker, A. Thompson, N. Sarwar, M. Caslake, A.S. Butterworth, P. Amouyel, G. Assmann, S.J. Bakker, E.L. Barr, E. BarrettConnor, E.J. Benjamin, C. Bjorkelund, H. Brenner, E. Brunner, R. Clarke,
J.A. Cooper, P. Cremer, M. Cushman, G.R. Dagenais, R.B. D'Agostino Sr. R. Dankner, G. Davey-Smith, D. Deeg, J.M. Dekker, G. Engstrom, A.R. Folsom, F.G. Fowkes, J. Gallacher, J.M. Gaziano, S. Giampaoli, R.F. Gillum, A. Hofman, B.V. Howard, E. Ingelsson, H. Iso, T. Jorgensen, S. Kiechl, A. Kitamura, Y. Kiyohara, W. Koenig, D. Kromhout, L.H. Kuller, D.A. Lawlor, T.W. Meade, A. Nissinen, B.G. Nordestgaard, A. Onat, D.B. Panagiotakos, B.M. Psaty, B. Rodriguez, A. Rosengren, V. Salomaa, J. Kauhanen, J.T. Salonen, J.A. Shaffer, S. Shea, I. Ford, C.D. Stehouwer, T.E. Strandberg, R.W. Tipping, A. Tosetto, S. Wassertheil-Smoller, P. Wennberg, R.G. Westendorp, P.H. Whincup, L. Wilhelmsen, M. Woodward, G.D. Lowe, N.J. Wareham, K.T. Khaw, N. Sattar, C.J. Packard, V. Gudnason, P.M. Ridker, M.B. Pepys, S.G. Thompson, J. Danesh, Creactive protein, fibrinogen, and cardiovascular disease prediction, N. Engl. J. Med. 367 (2012) 1310-1320.

[535] J. Zacho, A. Tybjaerg-Hansen, J.S. Jensen, P. Grande, H. Sillesen, B.G. Nordestgaard, Genetically elevated C-reactive protein and ischemic vascular disease, N. Engl. J. Med. 359 (2008) 1897-1908.

[536] D.T. Miller, R.Y. Zee, J. Suk Danik, P. Kozlowski, D.I. Chasman, R. Lazarus, N.R. Cook, P.M. Ridker, D.J. Kwiatkowski, Association of common CRP gene variants with CRP levels and cardiovascular events, Ann. Hum. Genet. 69 (2005) 623-638.

[537] W. Koenig, High-sensitivity C-reactive protein and atherosclerotic disease: from improved risk prediction to risk-guided therapy, Int. J. Cardiol. 168 (2013) 5126-5134.

[538] S.M. Grundy, N.J. Stone, A.L. Bailey, C. Beam, K.K. Birtcher, R.S. Blumenthal, L.T. Braun, L.T. Braun, S. de Ferranti, J. Faiella-Tommasino, D.E. Forman, R. Goldberg, P.A. Heidenreich, M.A. Hlatky, D.W. Jones, D. Lloyd-Jones, N. LopezPajares, C.E. Ndumele, C.E. Orringer, C.A. Peralta, J.J. Saseen, S.C. Smith Jr., L. Sperling, S.S. Virani, J. Yeboah, AHA/ACC/AACVPR/AAPA/ABC/ACPM/ADA/ AGS/APhA/ASPC/NLA/PCNA guideline on the management of blood cholesterol: executive summary: a report of the American College of Cardiology/American Heart Association Task Force on Clinical Practice Guidelines, J. Am. Coll. Cardiol. (2018) 2018:S0735-1097:39033-39038.

[539] C. Arnaud, F. Burger, S. Steffens, N.R. Veillard, T.H. Nguyen, D. Trono, F. Mach, Statins reduce interleukin-6-induced C-reactive protein in human hepatocytes: new evidence for direct antiinflammatory effects of statins, Arterioscler. Thromb. Vasc. Biol. 25 (2005) 1231-1236.

[540] E.A. Bohula, R.P. Giugliano, C.P. Cannon, J. Zhou, S.A. Murphy, J.A. White, A.M. Tershakovec, M.A. Blazing, E. Braunwald, Achievement of dual low-density lipoprotein cholesterol and high-sensitivity C-reactive protein targets more frequent with the addition of ezetimibe to simvastatin and associated with better outcomes in IMPROVE-IT, Circulation 132 (2015) 1224-1233.

[541] P.M. Ridker, C.P. Cannon, D. Morrow, N. Rifai, L.M. Rose, C.H. McCabe, M.A. Pfeffer, E. Braunwald, Pravastatin or Atorvastatin Evaluation and Infection Therapy-Thrombolysis in Myocardial Infarction 22 (PROVE IT-TIMI 22) Investigators. C-reactive protein levels and outcomes after statin therapy, N. Engl. J. Med. 352 (2005) 20-28.

[542] P.M. Ridker, E. Danielson, F.A. Fonseca, J. Genest, A.M. Gotto Jr., J.J. Kastelein, W. Koenig, P. Libby, A.J. Lorenzatti, J.G. MacFadyen, B.G. Nordestgaard, J. Shepherd, J.T. Willerson, R.J. Glynn, JUPITER Study Group, Rosuvastatin to prevent vascular events in men and women with elevated C-reactive protein, N. Engl. J. Med. 359 (2008) 2195-2207.

[543] P.M. Ridker, N. Rifai, M. Clearfield, J.R. Downs, S.E. Weis, J.S. Miles, A.M. Gotto Jr.Air Force/Texas Coronary Atherosclerosis Prevention Study Investigators, Measurement of C-reactive protein for the targeting of statin therapy in the primary prevention of acute coronary events, N. Engl. J. Med. 344 (2001) 1959-1965.

[544] P.M. Ridker, N. Rifai, M.A. Pfeffer, F.M. Sacks, L.A. Moye, S. Goldman, G.C. Flaker, E. Braunwald, Inflammation, pravastatin, and the risk of coronary events after myocardial infarction in patients with average cholesterol levels. Cholesterol and Recurrent Events (CARE) Investigators, Circulation 98 (1998) 839-844.

[545] P.M. Ridker, E. Danielson, F.A. Fonseca, J. Genest, A.M. Gotto Jr., J.J. Kastelein, W. Koenig, P. Libby, A.J. Lorenzatti, J.G. Macfadyen, B.G. Nordestgaard, J. Shepherd, J.T. Willerson, R.J. Glynn, JUPITER Trial Study Group, Reduction in C-reactive protein and LDL cholesterol and cardiovascular event rates after initiation of rosuvastatin: a prospective study of the JUPITER trial, Lancet 373 (2009) 1175-1182.

[546] S.P. Barbosa, L.C. Lins, F.A. Fonseca, L.N. Matos, A.C. Aguirre, H.T. Bianco, J.B. Amaral, C.N. Franca, J.M. Santana, M.C. Izar, Effects of ezetimibe on markers of synthesis and absorption of cholesterol in high-risk patients with elevated Creactive protein, Life Sci. 92 (2013) 845-851.

[547] A. Sahebkar, P. Di Giosia, C.A. Stamerra, D. Grassi, C. Pedone, G. Ferretti, T. Bacchetti, C. Ferri, P. Giorgini, Effect of monoclonal antibodies to PCSK9 on high-sensitivity C-reactive protein levels: a meta-analysis of 16 randomized controlled treatment arms, Br. J. Clin. Pharmacol. 81 (2016) 1175-1190.

[548] P.M. Ridker, B.M. Everett, T. Thuren, J.G. MacFadyen, W.H. Chang, C. Ballantyne, F. Fonseca, J. Nicolau, W. Koenig, S.D. Anker, J.J.P. Kastelein, J.H. Cornel, P. Pais, D. Pella, J. Genest, R. Cifkova, A. Lorenzatti, T. Forster, Z. Kobalava, L. VidaSimiti, M. Flather, H. Shimokawa, H. Ogawa, M. Dellborg, P.R.F. Rossi, R.P.T. Troquay, P. Libby, R.J. Glynn, CANTOS Trial Group, Antiinflammatory therapy with canakinumab for atherosclerotic disease, N. Engl. J. Med. 377 (2017) 1119-1131.

[549] P.M. Ridker, Testing the inflammatory hypothesis of atherothrombosis: scientific rationale for the cardiovascular inflammation reduction trial (CIRT), J. Thromb. Haemost. 7 (2009) 332-339.

[550] P.M. Ridker, B.M. Everett, A. Pradhan, J.G. MacFadyen, D.H. Solomon, E. Zaharris, V. Mam, A. Hasan, Y. Rosenberg, E. Iturriaga, M. Gupta, M. Tsigoulis, S. Verma, 
M. Clearfield, P. Libby, S.Z. Goldhaber, R. Seagle, C. Ofori, M. Saklayen, S. Butman, N. Singh, M. Le May, O. Bertrand, J. Johnston, N.P. Paynter, R.J. GlynnCIRT Investigators, Low-dose methotrexate for the prevention of atherosclerotic events, N. Engl. J. Med. 380 (2019) 752-762.

[551] C. Weber, L. Badimon, F. Mach, E.P.C. van der Vorst, Therapeutic strategies for atherosclerosis and atherothrombosis: past, present and future, Thromb. Haemost. 117 (2017) 1258-1264.

[552] C. Newman, J. Tsai, M. Szarek, D. Luo, E. Gibson, Comparative safety of atorvastatin $80 \mathrm{mg}$ versus $10 \mathrm{mg}$ derived from analysis of 49 completed trials in 14,236 patients, Am. J. Cardiol. 97 (2006) 61-67.

[553] H. Bays, D.E. Cohen, N. Chalasani, S.A. Harrison, The National Lipid Association's Statin Safety Task Force. An assessment by the Statin Liver Safety Task Force: 2014 update, J Clin Lipidol 8 (2014) S47-S57.

[554] E. Bjornsson, E.I. Jacobsen, E. Kalaitzakis, Hepatotoxicity associated with statins: reports of idiosyncratic liver injury post-marketing, J. Hepatol. 56 (2012) 374-380.

[555] A.T. Clarke, P.C. Johnson, G.C. Hall, I. Ford, P.R. Mills, High dose atorvastatin associated with increased risk of significant hepatotoxicity in comparison to simvastatin in UK GPRD cohort, PLoS One 11 (2016) e0151587.

[556] H. Cederberg, A. Stancakova, N. Yaluri, S. Modi, J. Kuusisto, M. Laakso, Increased risk of diabetes with statin treatment is associated with impaired insulin sensitivity and insulin secretion: a 6 year follow-up study of the METSIM cohort, Diabetologia 58 (2015) 1109-1117.

[557] S. Mora, R.J. Glynn, J. Hsia, J.G. MacFadyen, J. Genest, P.M. Ridker, Statins for the primary prevention of cardiovascular events in women with elevated highsensitivity C-reactive protein or dyslipidemia: results from the Justification for the Use of Statins in Prevention: An Intervention Trial Evaluating Rosuvastatin (JUPITER) and meta-analysis of women from primary prevention trials, Circulation 121 (2010) 1069-1077.

[558] A. Atlas Writing GroupTimmis, N. Townsend, C. Gale, R. Grobbee, N. Maniadakis, M. Flather, E. Wilkins, L. Wright, R. Vos, J. Bax, M. Blum, F. Pinto, P. Vardas, European Society of Cardiology: cardiovascular disease statistics 2017, Eur. Heart J. 39 (2018) 508-579.

[559] G.A. Roth, M.H. Forouzanfar, A.E. Moran, R. Barber, G. Nguyen, V.L. Feigin, M. Naghavi, G.A. Mensah, C.J. Murray, Demographic and epidemiologic drivers of global cardiovascular mortality, N. Engl. J. Med. 372 (2015) 1333-1341.

[560] T. Aspelund, V. Gudnason, B.T. Magnusdottir, K. Andersen, G. Sigurdsson, B. Thorsson, L. Steingrimsdottir, J. Critchley, K. Bennett, M. O'Flaherty, S. Capewell, Analysing the large decline in coronary heart disease mortality in the Icelandic population aged 25-74 between the years 1981 and 2006, PLoS One 5 (2010) e13957.

[561] L. Bjorck, A. Rosengren, K. Bennett, G. Lappas, S. Capewell, Modelling the decreasing coronary heart disease mortality in Sweden between 1986 and 2002, Eur. Heart J. 30 (2009) 1046-1056.

[562] M. Pereira, A. Azevedo, N. Lunet, H. Carreira, M. O'Flaherty, S. Capewell, K. Bennett, Explaining the decline in coronary heart disease mortality in Portugal between 1995 and 2008, Circ Cardiovasc Qual Outcomes 6 (2013) 634-642.

[563] B. Unal, K. Sozmen, H. Arik, G. Gerceklioglu, D.U. Altun, H. Simsek, S. Doganay, Y. Demiral, O. Aslan, K. Bennett, M. O'Flaherty, S. Capewell, J. Critchley, Explaining the decline in coronary heart disease mortality in Turkey between 1995 and 2008, BMC Public Health 13 (2013) 1135.

[564] EunetHta Joint Action 2, Work Package 7, Subgroup. 3, E. Heintz, A. Gerber-Grote, S. Ghabri, F.F. Hamers, V.P. Rupel, R. Slabe-Erker, T. Davidson, Is there a European view on health economic evaluations? Results from a synopsis of methodological guidelines used in the EUnetHTA partner countries, PharmacoEconomics 34 (2016) 59-76.

[565] M. Drummond, M. Barbieri, J. Cook, H.A. Glick, J. Lis, F. Malik, S.D. Reed, F. Rutten, M. Sculpher, J. Severens, Transferability of economic evaluations across jurisdictions: ISPOR Good Research Practices Task Force report, Value Health 12 (2009) 409-418.

[566] T.R. Frieden, A framework for public health action: the health impact pyramid, Am. J. Public Health 100 (2010) 590-595.

[567] J.W. Hotchkiss, C.A. Davies, R. Dundas, N. Hawkins, P.S. Jhund, S. Scholes, M. Bajekal, M. O'Flaherty, J. Critchley, A.H. Leyland, S. Capewell, Explaining trends in Scottish coronary heart disease mortality between 2000 and 2010 using IMPACTSEC model: retrospective analysis using routine data, BMJ 348 (2014) g1088.

[568] L.J. Cobiac, A. Magnus, S. Lim, J.J. Barendregt, R. Carter, T. Vos, Which interventions offer best value for money in primary prevention of cardiovascular disease? PLoS One 7 (2012) e41842.

[569] M. Collins, H. Mason, M. O'Flaherty, M. Guzman-Castillo, J. Critchley, S. Capewell, An economic evaluation of salt reduction policies to reduce coronary heart disease in England: a policy modeling study, Value Health 17 (2014) 517-524.

[570] H. Mason, A. Shoaibi, R. Ghandour, M. O'Flaherty, S. Capewell, R. Khatib, S. Jabr, B. Unal, K. Sozmen, C. Arfa, W. Aissi, H. Ben Romdhane, F. Fouad, R. Al-Ali, A. Husseini, MedCHAMPS project team. A cost effectiveness analysis of salt reduction policies to reduce coronary heart disease in four Eastern Mediterranean countries, PLoS One 9 (2014) e84445.

[571] P.V. Moreira, L.G. Baraldi, J.C. Moubarac, C.A. Monteiro, A. Newton, S. Capewell, M. O'Flaherty, Comparing different policy scenarios to reduce the consumption of ultra-processed foods in UK: impact on cardiovascular disease mortality using a modelling approach, PLoS One 10 (2015) e0118353.

[572] C. O'Keeffe, Z. Kabir, M. O'Flaherty, J. Walton, S. Capewell, I.J. Perry, Modelling the impact of specific food policy options on coronary heart disease and stroke deaths in Ireland, BMJ Open 3 (2013) e002837.

[573] P. Barton, L. Andronis, A. Briggs, K. McPherson, S. Capewell, Effectiveness and cost effectiveness of cardiovascular disease prevention in whole populations: modelling study, BMJ 343 (2011) d4044.

[574] P.A. Muennig, M. Epstein, G. Li, C. DiMaggio, The cost-effectiveness of New York City's Safe Routes to School Program, Am. J. Public Health 104 (2014) 1294-1299.

[575] L. Roux, M. Pratt, T.O. Tengs, M.M. Yore, T.L. Yanagawa, J. Van Den Bos, C. Rutt, R.C. Brownson, K.E. Powell, G. Heath, H.W. Kohl III, S. Teutsch, J. Cawley, I.M. Lee, L. West, D.M. Buchner, Cost effectiveness of community-based physical activity interventions, Am. J. Prev. Med. 35 (2008) 578-588.

[576] T. Jørgensen, S. Capewell, E. Prescott, S. Allender, S. Sans, T. Zdrojewski, D. De Bacquer, J. de Sutter, O.H. Franco, S. Løgstrup, M. Volpe, S. Malyutina, P. Marques-Vidal, Z. Reiner, G.S. Tell, W.M. Verschuren, D. Vanuzzo, P.E.P. section of EACPR, Population-level changes to promote cardiovascular health, Eur J Prev Cardiol 20 (2013) 409-421.

[577] D. Mozaffarian, L.J. Appel, L. Van Horn, Components of a cardioprotective diet: new insights, Circulation 123 (2011) 2870-2891.

[578] K. Dalziel, L. Segal, Time to give nutrition interventions a higher profile: costeffectiveness of 10 nutrition interventions, Health Promot. Int. 22 (2007) 271-283.

[579] A.L. Ahern, G.M. Wheeler, P. Aveyard, E.J. Boyland, J.C.G. Halford, A.P. Mander, J. Woolston, A.M. Thomson, M. Tsiountsioura, D. Cole, B.R. Mead, L. Irvine, D. Turner, M. Suhrcke, L. Pimpin, L. Retat, A. Jaccard, L. Webber, S.R. Cohn, S.A. Jebb, Extended and standard duration weight-loss programme referrals for adults in primary care (WRAP): a randomised controlled trial, Lancet 389 (2017) 2214-2225.

[580] A. Neumann, L. Lindholm, M. Norberg, O. Schoffer, S.J. Klug, F. Norstrom, The cost-effectiveness of interventions targeting lifestyle change for the prevention of diabetes in a Swedish primary care and community based prevention program, Eur. J. Health Econ. 18 (2017) 905-919.

[581] M. Hoogendoorn, T.L. Feenstra, R.T. Hoogenveen, M.P. Rutten-van Molken, Longterm effectiveness and cost-effectiveness of smoking cessation interventions in patients with COPD, Thorax 65 (2010) 711-718.

[582] M. Forster, J.L. Veerman, J.J. Barendregt, T. Vos, Cost-effectiveness of diet and exercise interventions to reduce overweight and obesity, Int. J. Obes. 35 (2011) 1071-1078.

[583] C.K. Chow, S. Jolly, P. Rao-Melacini, K.A. Fox, S.S. Anand, S. Yusuf, Association of diet, exercise, and smoking modification with risk of early cardiovascular events after acute coronary syndromes, Circulation 121 (2010) 750-758.

[584] E. Loveman, G.K. Frampton, J. Shepherd, J. Picot, K. Cooper, J. Bryant, K. Welch, A. Clegg, The clinical effectiveness and cost-effectiveness of long-term weight management schemes for adults: a systematic review, Health Technol. Assess. 15 (2011) 1-182.

[585] C. Guerriero, J. Cairns, I. Roberts, A. Rodgers, R. Whittaker, C. Free, The costeffectiveness of smoking cessation support delivered by mobile phone text messaging: Txt2stop, Eur. J. Health Econ. 14 (2013) 789-797.

[586] A. McConnachie, A. Walker, M. Robertson, L. Marchbank, J. Peacock, C.J. Packard, S.M. Cobbe, I. Ford, Long-term impact on healthcare resource utilization of statin treatment, and its cost effectiveness in the primary prevention of cardiovascular disease: a record linkage study, Eur. Heart J. 35 (2014) 290-298.

[587] P. Peura, J. Martikainen, E. Soini, T. Hallinen, L. Niskanen, Cost-effectiveness of statins in the prevention of coronary heart disease events in middle-aged Finnish men, Curr. Med. Res. Opin. 24 (2008) 1823-1832.

[588] D.J. Heller, P.G. Coxson, J. Penko, M.J. Pletcher, L. Goldman, M.C. Odden, D.S. Kazi, K. Bibbins-Domingo, Evaluating the impact and cost-effectiveness of statin use guidelines for primary prevention of coronary heart disease and stroke, Circulation 136 (2017) 1087-1098.

[589] A. Pandya, S. Sy, S. Cho, M.C. Weinstein, T.A. Gaziano, Cost-effectiveness of 10year risk thresholds for initiation of statin therapy for primary prevention of cardiovascular disease, J. Am. Med. Assoc. 314 (2015) 142-150.

[590] C. Heart Protection Study, B. Mihaylova, A. Briggs, J. Armitage, S. Parish, A. Gray, R. Collins, Lifetime cost effectiveness of simvastatin in a range of risk groups and age groups derived from a randomised trial of 20,536 people, BMJ 333 (2006) 1145.

[591] S. Ward, M. Lloyd Jones, A. Pandor, M. Holmes, R. Ara, A. Ryan, W. Yeo, N. Payne, A systematic review and economic evaluation of statins for the prevention of coronary events, Health Technol. Assess. 11 (2007) 1-160 iii-iv).

[592] G.M. Davies, A. Vyas, C.A. Baxter, Economic evaluation of ezetimibe treatment in combination with statin therapy in the United States, J. Med. Econ. 20 (2017) 723-731.

[593] P. Lindgren, J. Graff, A.G. Olsson, T.J. Pedersen, B. JonssonIDEAL Trial Investigators, Cost-effectiveness of high-dose atorvastatin compared with regular dose simvastatin, Eur. Heart J. 28 (2007) 1448-1453.

[594] M.C. Stam-Slob, Y. van der Graaf, J.P. Greving, J.A. Dorresteijn, F.L. Visseren, Cost-effectiveness of intensifying lipid-lowering therapy with statins based on individual absolute benefit in coronary artery disease patients, J Am Heart Assoc 6 (2017) e004648.

[595] K. Kotseva, D. De Bacquer, G. De Backer, L. Ryden, C. Jennings, V. Gyberg, A. Abreu, C. Aguiar, A.C. Conde, K. Davletov, M. Dilic, M. Dolzhenko, D. Gaita, B. Georgiev, N. Gotcheva, N. Lalic, A. Laucevicius, D. Lovic, S. Mancas, D. Milicic, R. Oganov, A. Pajak, N. Pogosova, Z. Reiner, D. Vulic, D. WoodOn Behalf of The Euroaspire Investigators, Lifestyle and risk factor management in people at high risk of cardiovascular disease. A report from the European Society of Cardiology European Action on Secondary and Primary Prevention by Intervention to Reduce Events (EUROASPIRE) IV cross-sectional survey in 14 European regions, Eur J Prev Cardiol 23 (2016) 2007-2018.

[596] S.B. Cherry, J.S. Benner, M.A. Hussein, S.S. Tang, M.B. Nichol, The clinical and economic burden of nonadherence with antihypertensive and lipid-lowering 
therapy in hypertensive patients, Value Health 12 (2009) 489-497.

[597] A. Vonbank, S. Agewall, K.P. Kjeldsen, B.S. Lewis, C. Torp-Pedersen, C. Ceconi, C. Funck-Brentano, J.C. Kaski, A. Niessner, J. Tamargo, T. Walther, S. Wassmann, G. Rosano, H. Schmidt, C.H. Saely, H. Drexel, Comprehensive efforts to increase adherence to statin therapy, Eur. Heart J. 38 (2017) 2473-2479.

[598] G. Corrao, L. Scotti, A. Zambon, G. Baio, F. Nicotra, V. Conti, S. Capri, E. Tragni, L. Merlino, A.L. Catapano, G. Mancia, Cost-effectiveness of enhancing adherence to therapy with statins in the setting of primary cardiovascular prevention. Evidence from an empirical approach based on administrative databases, Atherosclerosis 217 (2011) 479-485.

[599] R.H. Chapman, S.L. Kowal, S.B. Cherry, C.P. Ferrufino, C.S. Roberts, L. Chen, The modeled lifetime cost-effectiveness of published adherence-improving interventions for antihypertensive and lipid-lowering medications, Value Health 13 (2010) 685-694.

[600] M.T. Brown, J.K. Bussell, Medication adherence: WHO cares? Mayo Clin. Proc. 86 (2011) 304-314.

\section{Further reading}

[30] M.W. Lorenz, J.F. Polak, M. Kavousi, E.B. Mathiesen, H. Volzke, T.P. Tuomainen, D. Sander, M. Plichart, A.L. Catapano, C.M. Robertson, S. Kiechl, T. Rundek, M. Desvarieux, L. Lind, C. Schmid, P. DasMahapatra, L. Gao, K. Ziegelbauer, M.L. Bots, S.G. Thompson, PROG-IMT Study Group, Carotid intima-media thickness progression to predict cardiovascular events in the general population (the PROGIMT collaborative project): a meta-analysis of individual participant data, Lancet 379 (2012) 2053-2062.

[138] D. Mozaffarian, A. Aro, W.C. Willett, Health effects of trans-fatty acids: experimental and observational evidence, Eur. J. Clin. Nutr. 63 (2009) S5-S21.

[139] P.M. Clifton, J.B. Keogh, A systematic review of the effect of dietary saturated and polyunsaturated fat on heart disease, Nutr. Metab. Cardiovasc. Dis. 27 (2017) 1060-1080.

[141] P.L. Hollaender, A.B. Ross, M. Kristensen, Whole-grain and blood lipid changes in apparently healthy adults: a systematic review and meta-analysis of randomized controlled studies, Am. J. Clin. Nutr. 102 (2015) 556-572.

[147] A.J. Nordmann, A. Nordmann, M. Briel, U. Keller, W.S. Yancy Jr., B.J. Brehm, H.C. Bucher, Effects of low-carbohydrate vs low-fat diets on weight loss and cardiovascular risk factors: a meta-analysis of randomized controlled trials, Arch. Intern. Med. 166 (2006) 285-293.

[149] S. Berger, G. Raman, R. Vishwanathan, P.F. Jacques, E.J. Johnson, Dietary cholesterol and cardiovascular disease: a systematic review and meta-analysis, Am. J. Clin. Nutr. 102 (2015) 276-294.

[150] J.D. Griffin, A.H. Lichtenstein, Dietary cholesterol and plasma lipoprotein profiles: randomized-controlled trials, Curr Nutr Rep 2 (2013) 274-282.

[152] D.W. Droste, C. Iliescu, M. Vaillant, M. Gantenbein, N. De Bremaeker, C. Lieunard, T. Velez, M. Meyer, T. Guth, A. Kuemmerle, G. Gilson, A. Chioti, A daily glass of red wine associated with lifestyle changes independently improves blood lipids in patients with carotid arteriosclerosis: results from a randomized controlled trial, Nutr. J. 12 (2013) 147.

[154] S. Yu-Poth, G. Zhao, T. Etherton, M. Naglak, S. Jonnalagadda, P.M. Kris-Etherton, Effects of the National Cholesterol Education Program's Step I and Step II dietary intervention programs on cardiovascular disease risk factors: a meta-analysis, Am. J. Clin. Nutr. 69 (1999) 632-646.

[155] F.L. Santos, S.S. Esteves, A. da Costa Pereira, W.S. Yancy Jr., J.P. Nunes, Systematic review and meta-analysis of clinical trials of the effects of low carbohydrate diets on cardiovascular risk factors, Obes. Rev. 13 (2012) 1048-1066.

[160] C. Gayet-Boyer, F. Tenenhaus-Aziza, C. Prunet, C. Marmonier, C. MalpuechBrugere, B. Lamarche, J.M. Chardigny, Is there a linear relationship between the dose of ruminant trans-fatty acids and cardiovascular risk markers in healthy subjects: results from a systematic review and meta-regression of randomised clinical trials, Br. J. Nutr. 112 (2014) 1914-1922.

[161] G.A. Kelley, K.S. Kelley, Impact of progressive resistance training on lipids and lipoproteins in adults: a meta-analysis of randomized controlled trials, Prev. Med. 48 (2009) 9-19.

[162] C.M. Kastorini, H.J. Milionis, K. Esposito, D. Giugliano, J.A. Goudevenos, D.B. Panagiotakos, The effect of Mediterranean diet on metabolic syndrome and its components: a meta-analysis of 50 studies and 534,906 individuals, J. Am. Coll. Cardiol. 57 (2011) 1299-1313.
[165] R. Micha, S. Khatibzadeh, P. Shi, S. Fahimi, S. Lim, K.G. Andrews, R.E. Engell, J. Powles, M. Ezzati, D. Mozaffarian, Global Burden of Diseases Nutrition and Chronic Diseases Expert Group NutriCoDE. Global, regional, and national consumption levels of dietary fats and oils in 1990 and 2010: a systematic analysis including 266 country-specific nutrition surveys, BMJ 348 (2014) g2272.

[166] L. Schwingshackl, B. Bogensberger, A. Bencic, S. Knuppel, H. Boeing, G. Hoffmann, Effects of oils and solid fats on blood lipids: a systematic review and network meta-analysis, J. Lipid Res. 59 (2018) 1771-1782.

[167] A.M. Dattilo, P.M. Kris-Etherton, Effects of weight reduction on blood lipids and lipoproteins: a meta-analysis, Am. J. Clin. Nutr. 56 (1992) 320-328.

[353] J. Schreml, I. Gouni-Berthold, Role of anti-PCSK9 antibodies in the treatment of patients with statin intolerance, Curr. Med. Chem. 25 (2018) 1538-1548.

[355] A.J. Vallejo-Vaz, R. Fayyad, S.M. Boekholdt, G.K. Hovingh, J.J. Kastelein, S. Melamed, P. Barter, D.D. Waters, K.K. Ray, Triglyceride-rich lipoprotein cholesterol and risk of cardiovascular events among patients receiving statin therapy in the TNT trial, Circulation 138 (2018) 770-781.

[356] A.L. Catapano, M. Farnier, J.M. Foody, P.P. Toth, J.E. Tomassini, P. Brudi, A.M. Tershakovec, Combination therapy in dyslipidemia: where are we now? Atherosclerosis 237 (2014) 319-335.

[361] L.E. Akioyamen, J. Genest, S.D. Shan, R.L. Reel, J.M. Albaum, A. Chu, J.V. Tu, Estimating the prevalence of heterozygous familial hypercholesterolaemia: a systematic review and meta-analysis, BMJ Open 7 (2017) e016461.

[388] T.A. Miettinen, K. Pyorala, A.G. Olsson, T.A. Musliner, T.J. Cook, O. Faergeman, K. Berg, T. Pedersen, J. Kjekshus, Cholesterol-lowering therapy in women and elderly patients with myocardial infarction or angina pectoris: findings from the Scandinavian Simvastatin Survival Study (4S), Circulation 96 (1997) 4211-4218.

[495] M. Barylski, S. Nikfar, D.P. Mikhailidis, P.P. Toth, P. Salari, K.K. Ray, M.J. Pencina, M. Rizzo, J. Rysz, M. Abdollahi, S.J. Nicholls, M. Banach, Lipid and Blood Pressure Meta-Analysis Collaboration Group. Statins decrease all-cause mortality only in CKD patients not requiring dialysis therapy-a meta-analysis of 11 randomized controlled trials involving 21,295 participants, Pharmacol. Res. 72 (2013) 35-44.

[496] S.C. Palmer, S.D. Navaneethan, J.C. Craig, D.W. Johnson, V. Perkovic, J. Hegbrant, G.F. Strippoli, HMG CoA reductase inhibitors (statins) for people with chronic kidney disease not requiring dialysis, Cochrane Database Syst. Rev. 5 (2014) CD007784.

[524] S. Subherwal, M.R. Patel, L. Kober, E.D. Peterson, D.L. Bhatt, G.H. Gislason, A.M. Olsen, W.S. Jones, C. Torp-Pedersen, E.L. Fosbol, Peripheral artery disease is a coronary heart disease risk equivalent among both men and women: results from a nationwide study, Eur J Prev Cardiol 22 (2015) 317-325.

[601] R.O. Bonow, R.A. Harrington, C.W. Yancy, Cost-effectiveness of PCSK9 inhibitors: proof in the modeling, JAMA Cardiol 2 (2017) 1298-1299.

[602] A. Arrieta, J.C. Hong, R. Khera, S.S. Virani, H.M. Krumholz, K. Nasir, Updated cost-effectiveness assessments of PCSK9 inhibitors from the perspectives of the health system and private payers: insights derived from the FOURIER trial, JAMA Cardiol 2 (2017) 1369-1374.

[603] G.C. Fonarow, A.C. Keech, T.R. Pedersen, R.P. Giugliano, P.S. Sever, P. Lindgren, B. van Hout, G. Villa, Y. Qian, R. Somaratne, M.S. Sabatine, Cost-effectiveness of evolocumab therapy for reducing cardiovascular events in patients with atherosclerotic cardiovascular disease, JAMA Cardiol 2 (2017) 1069-1078.

[604] M. Korman, T. Wisloff, Modelling the cost-effectiveness of PCSK9 inhibitors vs. ezetimibe through LDL-C reductions in a Norwegian setting, Eur Heart J Cardiovasc Pharmacother 4 (2018) 15-22.

[605] L. Annemans, C.J. Packard, A. Briggs, K.K. Ray, 'Highest risk-highest benefit' strategy: a pragmatic, cost-effective approach to targeting use of PCSK9 inhibitor therapies, Eur. Heart J. 39 (2018) 2546-2550.

[606] J.G. Robinson, R. Huijgen, K. Ray, J. Persons, J.J. Kastelein, M.J. Pencina, Determining when to add nonstatin therapy: a quantitative approach, J. Am. Coll. Cardiol. 68 (2016) 2412-2421.

[607] D.S. Kazi, J. Penko, P.G. Coxson, A.E. Moran, D.A. Ollendorf, J.A. Tice, K. BibbinsDomingo, Updated cost-effectiveness analysis of PCSK9 inhibitors based on the results of the FOURIER trial, J. Am. Med. Assoc. 318 (2017) 748-750.

[608] D.A. Wood, K. Kotseva, S. Connolly, C. Jennings, A. Mead, J. Jones, A. Holden, D. De Bacquer, T. Collier, G. De Backer, O. FaergemanEUROACTION Study Group, Nurse-coordinated multidisciplinary, family-based cardiovascular disease prevention programme (EUROACTION) for patients with coronary heart disease and asymptomatic individuals at high risk of cardiovascular disease: a paired, clusterrandomised controlled trial, Lancet 371 (2008) 1999-2012. 